\title{
SQUARE-WAVE VOLTAMMETRY \\ DETECTION FOR CAPILLARY \\ ELECTROPHORESIS
}

\author{
by \\ Geoff C. Gerhardt \\ A thesis submitted to the College of Graduate Studies and Research in partial fultillment \\ of the requirements for the degree of \\ Doctor of Philosophy in the Department of Chemistry \\ Lniversity of Saskatchewan \\ Saskatoon
}

Summer 1999

(c) Copıright Geoff Gerhardt. 1999. All rights reserved. 
National Library

of Canada

Acquisitions and Bibliographic Services

395 Wellington Street Ottawa ON K1A ONA Canada
Bibliothèque nationale du Canada

Acquisitions et services bibliographiques

395, ne Wellington

Ottawa ON K1 A ONA

Canada
The author has granted a nonexclusive licence allowing the National Library of Canada to reproduce, loan, distribute or sell copies of this thesis in microform, paper or electronic formats.

The author retains ownership of the copyright in this thesis. Neither the thesis nor substantial extracts from it may be printed or otherwise reproduced without the author's permission.
L'auteur a accordé une licence non exclusive permettant à la Bibliothèque nationale du Canada de reproduire, prêter, distribuer ou vendre des copies de cette thèse sous la forme de microfiche/film, de reproduction sur papier ou sur format électronique.

L'auteur conserve la propriété du droit d'auteur qui protège cette thèse. $\mathrm{Ni}$ la thèse ni des extraits substantiels de celle-ci ne doivent être imprimés ou autrement reproduits sans son autorisation. 


\title{
UNIVERSITY OF SASKATCHEWAN
}

\section{College of Graduate Studies and Research \\ SUMMARY OF DISSERTATION}

Submitted in partial fulfillment

of the requirements for the

\section{DEGREE OF DOCTOR OF PHILOSOPHY}

\author{
By \\ Geoff Gerhardt \\ Department of Chemistry \\ University of Saskatchewan
}

Fall 1999

Examining Committee:

Dr. S. Koustov

Physics \& Engineering Physics

Dr. A.S. Baranski

Dr. R. M. Cassidy

Dr. J.D. MacNeil

Dr. D.A. Durden

External Examiner:

Edgar Arriaga

Department of Chemistry

University of Minnesota

A-1, 139 Smith Hall

207 Pleasant St. SE

Minneapolis, MN 55455-0431
Dean's Designate, Chair

College of Graduate Studies and Research

Chair of Advisory Committee, Dept. of Chemistry

Supervisor, Department of Chemistry

Canadian Food Inspection Agency

Neuropsychiatric Research Unit 


\section{PERMISSION TO USE}

In presenting this thesis in partial fulfillment of the requirements of a Postgraduate degree from the University of Saskatchewan, I agree that the Libraries of this University may make it freely available for inspection. I further agree that permission for copying of this thesis in any manner, in whole or in part, for scholarly purposes may be granted by the professor or professors who supervised my thesis work or, in their absence, by the Head of the Department or the Dean of the College in which my thesis work was done. It is understood that any copying or publication or use of this thesis or parts thereof for financial gain shall not be allowed without my written permission. It is also understood that due recognition shall be given to me and to the University of Saskatchewan in any scholarly use which may be made of any materials in my thesis.

Requests for permission to copy or to make other use of material in this thesis in whole or part should be addressed to:

Head of the Department of Chemistry

University of Saskatchewan

Saskatoon, Saskatchewan 


\section{ABSTRACT}

While the development and application of capillary electrophoresis (CE) has matured significantly in the last two decades, its application to trace analysis has been limited by the lack of a generally applicable, sensitive detection method. This Ph.D. project's aim was to develop such a method using electrochemical detection. After an initial review of the work done in electrochemical detection (ECD) for CE, it was determined that of the systems that had been reported, most had significant limitations for practical use. These systems were typically fragile and difficult to construct and were applicable to only electrochemically active analytes. Given this, a simplified and more robust CE-ECD instrumentation system was developed. This system comprised of a compact CE-ECD cell that allowed easy and secure alignment of the capillary and electrode and a flexible data acquisition/control system capable of investigating a number of ECD modes (both amperometric and voltammetric). Because difficulties were encountered using this CE-ECD cell in commercial automated CE systems, several automated CE instruments were constructed in-house. These systems included an addressable sample/buffer vial tray, vial lifting/pressure rinsing system that allowed unattended automated CE-ECD runs. Using this instrumentation, an ECD system based on square-wave voltammetry was developed (CE-SWV). Detection limits obtained with CE-SWV for neurotransmitters ( $150 \mathrm{nM}, \mathrm{S} / \mathrm{N}=3$ ) were comparable to those obtained using more fragile and complex amperometric CE detection systems. While CE-SWV worked well for detection of electroactive analytes (redox processes), one of the reasons for 
investigating SWV was to datermine whether it could detect other electrode processes such as analyte adsorption to develop a more generally applicable CE detection method. Using SWV, changes in the background electrolyte response were observed as organic analytes exited the capillary and interacted with a Pt electrode. Initially, for non-aromatic analytes, these changes appeared to result from physical adsorption of the analyte which "blocked" the hydrogen adsorption/reduction normally observed and produced negative electropherogram peaks. Using this as a detection method, reasonable detection limits ( I $\mu \mathrm{M}, \mathrm{S} / \mathrm{N}=3$ ) were obtained for analytes that did not have $\mathrm{UV}$-chromophores.

When this detection method was applied to analytes with $\pi$-electron density (i.e. analytes with phenyl or carbonyl functionality), positive SWV responses were observed, indicating an additional chemisorption process was involved. Positive voltage pulses ( $400 \mathrm{mV}$ for 5 ms) applied before the SWV scan were found to be necessary to elicit this positive response. It appeared that these positive voltage pulses oxidized the Pt electrode, possibly creating a reactive $\mathrm{Pt}-\mathrm{OH}$ layer which catalyzed oxidation of these organic analytes which were then reduced during the negative-going SWV scan used. These positive voltage pulses also served to maintain a clean electrode surface to prevent electrode fouling - a common problem encountered in ECD techniques. This positive SWV response, when plotted versus migration time, resulted in detection limits $(5 \mathrm{nM}, \mathrm{S} / \mathrm{N}=3)$ over an order of magnitude better than UV detection for analytes with good UV chromophores. This detection method, termed adsorption-based electrochemical detection (AdsECD), was demonstrated to be applicable to a wide variety of important organic analytes (over 50 organic analytes previously considered to be electrochemically inactive were detected) 
separated by CE using a variety of separation systems (i.e. separation systems with varied $\mathrm{pH}$, ionic strength, micellar systems, added organic solvents and chiral selectors were investigated). Coupled with the rugged and easy-to-use CE-ECD instrumentation that was developed, AdsECD was shown to be a technique that should prove to be useful for a wide variety of practical chemical analysis problems. 


\section{DEDICATION}

Thank you Richard and Jon. If you ever find yourselves wondering whether you made an impact in this world, phone me.

Thank you, Anna, Anthony and Thomas for keeping me centered and giving me focus. 


\section{TABLE OF CONTENTS}

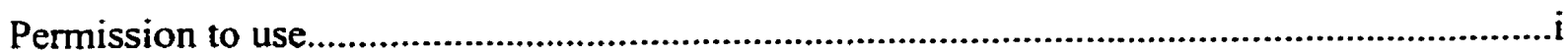

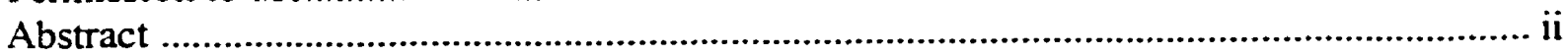

Dedication ...................................................................................................................

Table of Contents ...................................................................................................................

List of figures ..................................................................................................................... viii

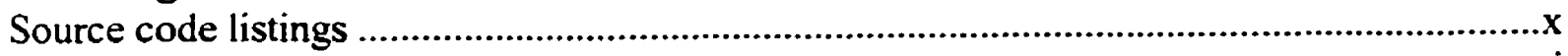

List of abbreviations...................................................................................................................

Thesis Introduction ...........................................................................................................

Chapter 1 - Development of robust instrumentation for CE-ECD ................................11

1.1 Introduction.......................................................................................................... 11

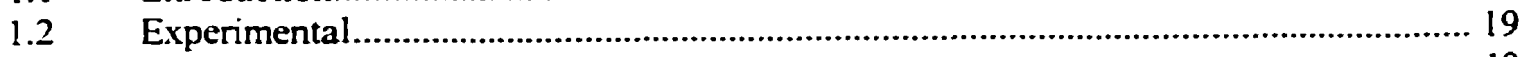

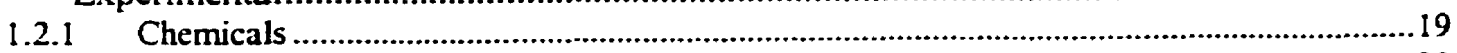

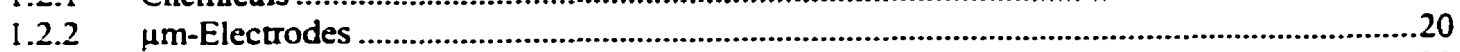

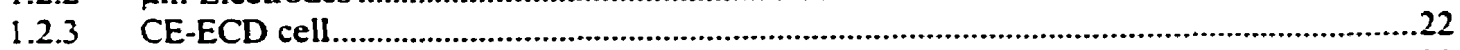

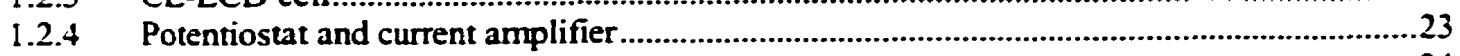

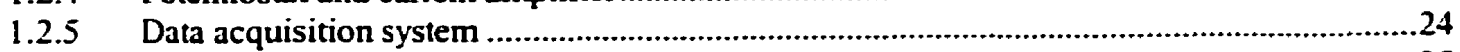

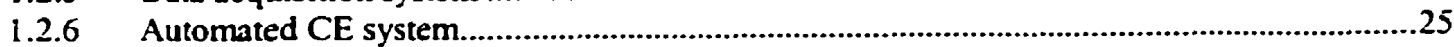

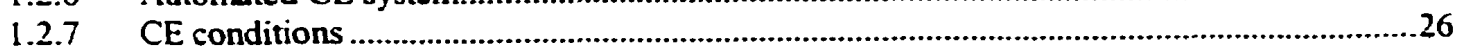

1.2.8 Calculations of $\mathrm{S} / \mathrm{N}$ and peak efficiency and asymmetry ................................................27

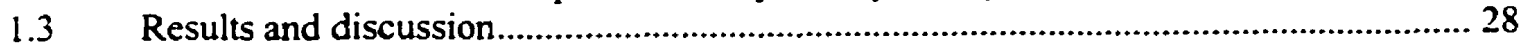

1.3.1 CE-ECD cell and $\mu \mathrm{m}$-scale electrode .....................................................................................

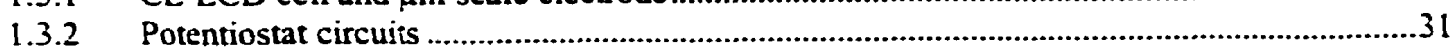

1.3.3 Effect of capillary-electrode distance and axial alignment .......................................................34

1.3.4 Effect of capillary intermal diameter .....................................................................................51

1.3.5 An improved electrode positioning system ……………………………………………….....53

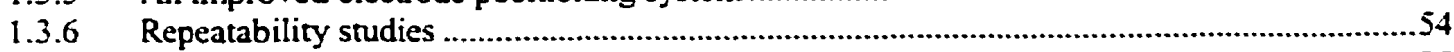

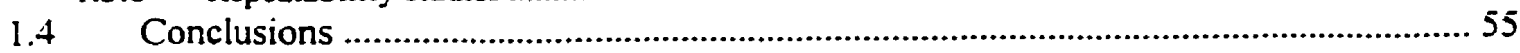

Chapter 2 - Square-wave voltammetry detection for CE .............................................57

2.1 Introduction...................................................................................................... 57

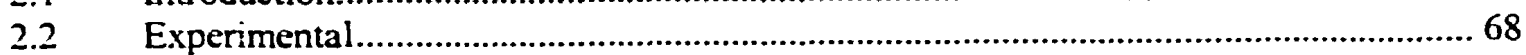

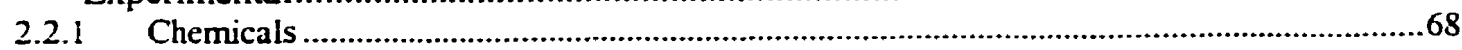

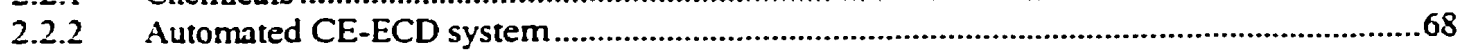

2.2 .3 CE Conditions ..........................................................................................................68

2.2.4 Calculations of $\mathrm{S} \mathrm{N}$ and peak efficiency ...........................................................................69

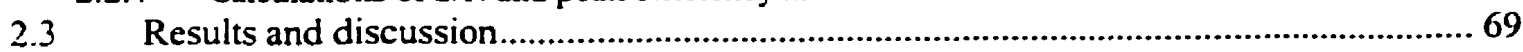

$2.3 .1 \quad$ Electrode type and positioning …………………………….................................................69

2.3.2 Square-wave voltammetry parameters ………………………………………………………....72

2.3.3 SWV raw data analysis ...................................................................................................76

2.3.4 Obtaining quantitative information from voltammograms .................................................. 78

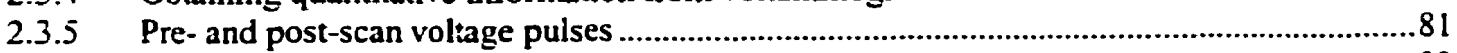

2.3.6 Fourier-transform filtering of voltammograms ...................................................................82

2.3.7 CE-SWV of Neurotransmitters...............................................................................................84

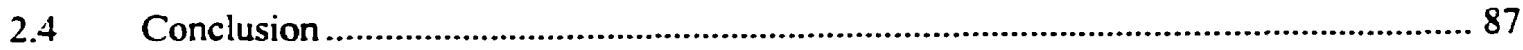

Chapter 3 - Adsorption-based electrochemical detection ..............................................89

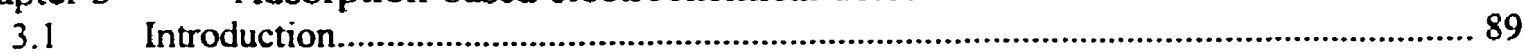

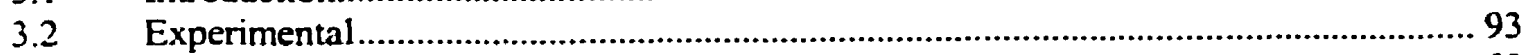

3.2.1 Chemicals ................................................................................................................................93 


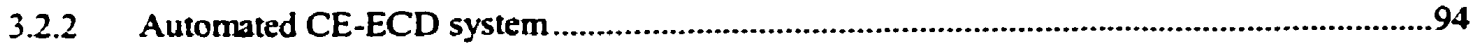

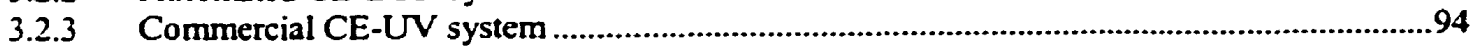

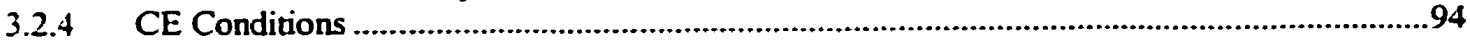

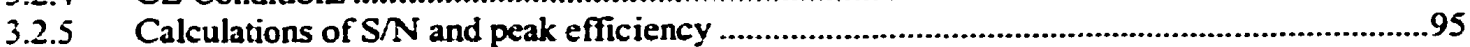

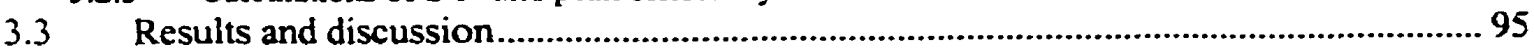

3.3.1 CE-SWV detection of adsorption-based responses for organic analytes ...........................95

3.3.2 Optimization of the CE-SWV waveform ......................................................................104

3.3.3 Post- and pre-SWV scan potential pulses......................................................................109

3.3.4 Effect of analyte structure on SWV response..........................................................118

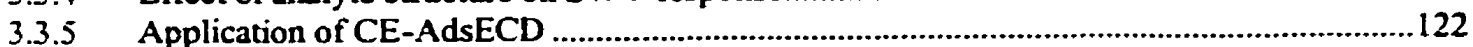

3.3.5.1 CE-AdsECD separations of basic drugs of forensic interest .................................. 122

3.3.5.2 CE-AdsECD enantiomeric separations of 2-HMP and Deprenyl............................124

3.3.5.3 CE-AdsECD separations of $\beta$-agonists used for illicit veterinary applications.........127

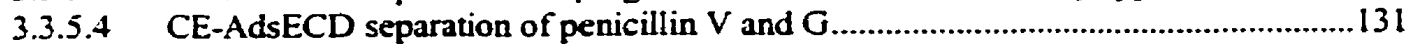

3.3.5.5 CE-AdsECD separation of peptides ..................................................................133

3.3.5.6 CE-AdsECD separation of nonsteroidal anti-inflammatory drugs ..........................134

3.3.6 Linearity of the AdsECD response ............................................................................... 137

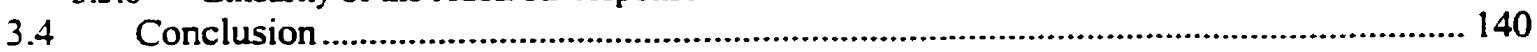

Thesis Conclusion .................................................................................................................143

Appendix A - Development of data acquisition/control systems ....................................146

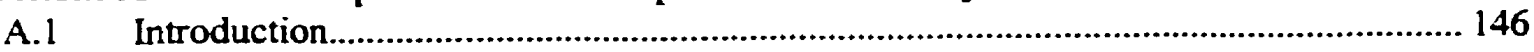

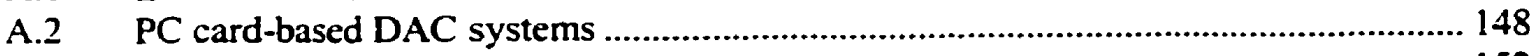

A.3 Microcontroller-based DAC systems........................................................ 152

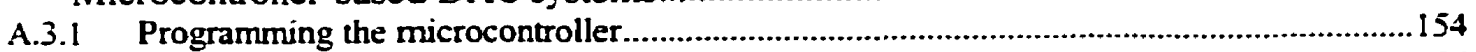

A.3.2 Parsing of SWV scan data sent from microcontroller ...................................................156

A.3.3 Quantitation of the voltammogram data to produce an electropherogram ........................ 158

A.3.4 Implications of using an 8-bit A-to-D converter for SWV current data collection .............159

A.4 Potentiostat and current follower circuits ............................................................... 163 


\section{LIST OF FIGURES}

Chapter 1.

Figure 1.1 - Working/auxiliary electrode assembly schematic. 20

Figure 1.2 - CE-ECD cell schematic.

Figure I.3 - Picture of CE-ECD cell. $\quad 22$

Figure 1.4 - Potentiostat and current amplifier schematic. $\quad 24$

Figure 1.5 - Picture of in-house constructed automated CE-ECD system.

Figure 1.6 - Schematic of instrument amplifier-type potentiostat 31

Figure 1.8 - Illustration of capillary-electrode misalignment (a) and distance (b). 34

Figure 1.7 - Voltammogram of background response observed for a Pt electrode in $100 \mathrm{mM}, \mathrm{pH} 7.0$ phosphate buffer.

Figure 1.9- Potential shift observed when separation voltage was applied and the capillary and electrode were intentionally misaligned.

Figure 1.10 - Effect of capillary-electrode distance on potential shift.

Figure 1.11 - Effect of capillary-electrode distance on trimethoprim peak shape. 40

Figure 1.12 - Effect capillary-electrode distance on separation efficiency and $\mathrm{S} / \mathrm{N}$.

Figure 1.13 - Effect of capillary-electrode distance on peak asymmetry.

Figure 1.14 - Effect of capillary-electrode misalignment on trimethoprim electropherogram peak. 44

Figure 1.15 - Effect of capillary-electrode alignment on the potential shift. 47

Figure 1.16 - Effect of capillary-electrode distance on the potential shift. $\quad 47$

Figure 1.17 - Effect of capillary-electrode distance on Deprenyl peak shape with a low-pH electrolyte. 48

Figure 1.18 - Effect of capillary-electrode distance on Deprenyl $\mathrm{S} / \mathrm{N}$ and efficiency. 49

Figure 1.19 - Effect of capillary-electrode alignment on Deprenyl peak shape with the low-pH electrolyte. 51

Figure 1.20 - Effect of capillary diameter on the separation of sulfonamide antibiotics. 52

Chapter 2.

Figure 2.1 - Pulsed voltammetric techniques.

Figure 2.2 - Background subtracted square wave voltammograms of dopamine and epinephrine. $\quad 70$

Figure 2.3 - The general SWV waveform used.

Figure 2.4 - Effect of square-wave frequency on dopamine $\mathrm{S} / \mathrm{N}$.

Figure 2.5 - Effect of square-wave amplitude on dopamine $\mathrm{S} / \mathrm{N}$.

Figure 2.6 - Effect of the amount of initial square-wave current response rejected on dopamine $S \wedge$. $\quad 77$

Figure 2.7 - Illustration of voltammogram quantitation techniques.

Figure 2.8 - Effect of number of points included in the running average on $\mathrm{S} / \mathrm{N}$ and peak efficiency. $\quad 80$

Figure 2.9 - Illustration of the impact of Fourier transform filtering on SWV. $\quad 82$

Figure 2.10 - Effect of Fourier transform filter on electropherogram noise. $\quad 83$

Figure 2.1 I - CE-SWV electropherogram of $5 \mu \mathrm{M}$ dopamine and epinephrine. $\quad 84$

Figure 2.12 - Calibration curves for dopamine and epinephrine.

Figure 2.13 - Sensitivity plots for dopamine and epinephrine. $\quad 86$

$\begin{array}{ll}\text { Figure 2.14 - 3-D plot of } 10 \mu \mathrm{M} \text { dopamine and epinephrine separation. } & 87\end{array}$ 
Chapter 3.

Figure 3.1 - Structure of dihydrostreptomycin. 96

Figure 3.2 - SWV response for $50 \mathrm{mM}$ pH 2.5 phosphate buffer with/without dihydrostreptomycin. 97

Figure 3.3 - Electropherogram of a dihydrostreptomycin CE separation. 98

Figure 3.4 - Structures of the sulfonamide antibiotics and trimethoprim. 99

Figure 3.5 - SWV response of background electrolyte with and without sulfamethzine $\quad 100$

Figure 3.6 - 3-D CE-AdsECD electropherogram of sulfonamide separation. 102

Figure 3.7 - 2-dimensional CE-AdsECD electropherogram of sulfonamide separation separation. 103

Figure 3.8 - CE-UV separation of sulfonamide antibiotics. 103

Figure 3.9 - Effect of square-wave frequency on sulfonamide S/N. 106

Figure 3.10 - Effect of square-wave amplitude on sulfonamide S/N. 107

Figure 3.11 - Effect of varied amount of initial square-wave response rejected on peak height. 108

Figure 3.12 - Effect of post-scan pulse potential on trimethoprim electropherogram peak shape. 110

Figure 3.13 - Effect of pre-scan pulse duration on sulfonamide S/N.

Figure 3.14 - Effect of post-scan potential on trimethoprim peak using new potentiostat configuration (electropherograms).

Figure 3.15 - Effect of post-scan potential on trimethoprim $\mathrm{S} / \mathrm{N}$ and peak efficiency using new potentiostat $\begin{array}{ll}\text { configuration ( } \mathrm{S} / \mathrm{N} \text { and efficiency plots). } & 116\end{array}$

Figure 3.16 - Structures of the amino acids.

$\begin{array}{ll}\text { Figure } 3.17 \text { - Background subtracted SWV response produced by amino acids. } & 120\end{array}$

$\begin{array}{ll}\text { Figure } 3.18 \text { - CE-AdsECD amino acid separation. } & 121\end{array}$

Figure 3.19 - CE-UV and CE-AdsECD separations of RCMP QC mixture..

Figure 3.20 - Structures of Deprenyl and 2-HMP. 125

Figure 3.21 - CE-AdsECD separation of Deprenyl and 2-HMP with/without $1 \% \beta$-cyclodextrin added. 126

$\begin{array}{ll}\text { Figure } 3.22 \text { - Structures of the } \beta \text {-agonists. } & 127\end{array}$

Figure 3.23 - CE-AdsECD separation of $\beta$-agonists. 129

Figure 3. 24 - CE-AdsECD separation of $\beta$-agonists compared to LC-MS-MS separation. 130

Figure 3.25 - Structures of penicillin V and G. 132

Figure 3.26 - CE-AdsECD separation of penicillins.

Figure 3.27 - CE-AdsECD separation of polypeptides. 134

Figure 3.28 - Structures of non-steroidal anti-inflammatory drugs. 135

Figure 3.29 - CE-AdsECD electropherogram of non-steroidal anti-inflammatory drugs. 136

Figure 3.30 - Calibration curves for the sulfonamide antibiotics. 137

Figure 3.31 - Sensitivity plots for the sulfonamide antibiotics. 138

Figure 3.32 - Calibration curves of the $\beta$-agonist drugs. 139

$\begin{array}{ll}\text { Figure } 3.33 \text { - Sensitivity plots of the } \beta \text {-agonist drugs. } & 140\end{array}$

Appendix A.

Figure A.1 - Screenshot of CE-SWV application using the National Instruments AT-MIO-E DAC card....151

Figure A.2 - Pin-out of 16C73 PIC microcontroller. ..................................................................152

Figure A.3 - Simulations illustrating the resolution enhancements produced by signal averaging..............162

Figure A.4 - Potentiostat/current follower circuit used in with the microcontroller-based DAC system....... 66 


\section{SOURCE CODE LISTINGS}

Appendix A.

Listing A.1 - x86 assembly language routine used to perform a linear DC voltage ramp. ........................167

Listing A.2 - PIC-C code to perform SWV waveform and data collection...................................................169

Listing A.3 - Delphi 4.0 code used to parse incoming scan data received from mictocontroller....................172

Listing A.4 - Delphi 4.0 code used to quantitate stored voltammograms. ..............................................174 


\section{LIST OF ABBREVIATIONS}

$\begin{array}{ll}\text { AdsECD } & \text { - Adsorption-based electrochemical detection } \\ \text { CE } & \text { - Capillary electrophoresis } \\ \text { ECD } & \text { - Electrochemical detection } \\ \text { GC } & \text { - Gas chromatography } \\ \text { HPLC } & \text { - High performance liquid chromatography } \\ \text { LIF } & \text { - Laser-induced fluorescence } \\ \text { MS } & \text { - Mass spectroscopy } \\ \text { TLC } & \text { - Thin-layer chromatography } \\ \text { SWV } & \text { - Square-wave voltammetry } \\ \text { CV } & \text { - Cyclic voltammetry }\end{array}$




\section{THESIS INTRODUCTION}

The development of analytical separation techniques such as high-performance liquid chromatography (HPLC) and gas chromatography (GC) and thin-layer chromatography (TLC) have given analytical chemists the ability to investigate complex samples. Although there are sensors for specific chemicals (ion selective electrodes, immunoassay techniques), independent detection of most analytes in complex mixtures usually requires a powerful separation technique. In biological matrices made up of thousands of chemical species, HPLC separation parameters can be adjusted to isolate and quantify single analytes. Similarly, GC has been used by the petroleum industry to determine the complex hydrocarbon mixtures found in the various processed crude oil products.

To achieve separation of a complex sample mixture, HPLC uses a flowing liquid phase moving through a column packed with a solid stationary phase. When a complex sample is injected at the head of the column, the components which make up this sample will travel through the column at rates which will depend upon their partition coefficient between the liquid and stationary phases. By manipulating the composition of the liquid and stationary phases to maximize the difference in the partition coefficients of these sample components, separation can be achieved as the sample zone moves through the column. A detector at the exit of the column is used to quantify analytes as they exit the column. The relatively low diffusion rate of molecules in liquids (between and into and 
out of particles) ultimately limits the separation efficiency of HPLC. Because pressurized fluid flow is characterized by a parabolic flow profile where flow velocity is lower near the flow stream walls and higher at mid-stream, smaller, more porous stationary phase particles have been used to reduce the distance analytes must travel across stationary phase channels (reducing the detrimental effects of parabolic flow) and therby increasing separation efficiency. However, as smaller stationary particle sizes are used, higher pressures are required to force the mobile phase through the columns. The limitation that this parabolic flow profile places on separation efficiency is one of the fundamental issues most liquid chromatographers wrestle with when trying to separate similar analytes. Other limitations of HPLC include:

- complex, expensive instrumentation required to produce high fluid pressures.

- high consumable cost (columns, solvents).

- use of large volumes of solvents results in a waste product that is costly to dispose of.

- difficulties encountered with polar and ionic analytes due to their interaction with the silica stationary phase supports that are commonly used.

GC also uses a fluid phase forced through a column, but in modern capillary GC the fluid is a gas and the stationary phase is usually coated on the wall of a capillary tube. Although the phase ratio (solid phase:liquid phase) is much lower in GC than HPLC and parabolic flow is still encountered, much higher separation efficiencies can be obtained, because much higher diffusion rates are observed in gas phases. Analytes can traverse the column diameter rapidly, thus sampling the various flow rates in the parabolic flow profile 
equally. Lower band broadening is observed which allows long capillaries to be used to achieve highly efficient separations. Despite this high separation efficiency, GC also has significant limitations. The most severe one is the requirement that analytes must be volatile enough to enter the gas phase. Polar and ionic species can not be separated using GC unless derivatized to increase their volatility. Although analyte volatility can be achieved using high temperatures, this only applies to analytes which remain stable at these elevated temperatures.

While HPLC and GC are the primary separation techniques analytical chemists turn to, TLC is also used. In TLC a thin stationary phase layer is applied to a glass sheet. A complex sample mixture is pipetted as a small zone onto the bottom of the plate and the bottom of the plate is immersed in mobile phase. The mobile phase will migrate up the plate by capillary action and separation of the complex sample based upon similar mechanisms found in HPLC will occur. Although TLC was once a popular separation/detection technique because of its low cost , because the separation efficiencies that can be obtained using TLC are poor and sensitive detection is difficult, it is currently more often used for sample clean-up or gross matrix separations.

While these traditional chromatographic separation techniques all involve partitioning of the analyte between stationary and mobile phases, separation of analytes based upon their behavior in an electric field has also been explored. Tiselius first reported the separation of complex mixtures by exploiting the differential movement of charged species in an electric field in $1937^{1}$ to observe the movement of protein mixtures 
in a buffer solution. In 1967 Hjertén performed electrophoresis in millimeter-scale internal diameters tubes ${ }^{2}$. The large currents that were generated within these tubes resulted in Joule heating which limited the separation efficiencies he could obtain even though he rotated these large-bore tubes along their longitudinal axis to minimize the effects of convection. It was not until 198 I when Jorgenson and Lukacs introduced modern capillary electrophoresis using $75 \mu \mathrm{m}$ capillaries ${ }^{3}$ that electrophoresis began to develop into a separation technique that could address some of the limitations of HPLC and GC.

When capillary tubes are used for electrophoretic separations, two types of movement are observed. In addition to the general movement of ions (at rates which depend on the charge/mass of the ion), which occurs in the presence of an electric field, a bulk fluid flow is generated at the walls of the capillary. This flow, termed electroosmotic flow (EOF), is generated at the walls of the capillary. The silica groups at the walls of fused-silica capillaries will, to varying degrees, have dissociated $\mathrm{Si}^{-} \mathrm{O}^{-}$groups. Positively charged ions in the electrolyte will be attracted to these negative wall charges by coulombic interactions resulting in a higher concentration of positive charges existing at the capillary walls. When a high separation potential is applied across the capillary, this "shell" of positive ions will migrate to the cathode, pulling the inner capillary contents along with it resulting in a bulk flow of the capillary contents. Because this flow is generated at the walls of the capillary it generates a very flat flow profile unlike pressuredriven flow which has a parabolic profile. Because of this flat flow profile, zone broadening is minimized resulting in high separation efficiencies. Charged analytes will 
move through the capillary with a velocity that is dependant upon both the EOF and their electrophoretic mobility. Where a high positive EOF exists (positive indicating movement towards detector), both positively and negatively charged species can have positive velocities and be analyzed in a single run.

There are several different modes of separation in CE. The simplest is manipulating the separation environment to maximize the electrophoretic mobility differences in the analytes of interest to obtain separation. Tarabe et $\mathrm{al}^{4}$ introduced the separation of neutral hydrophobic analytes, in 1984, using a technique termed micellar electrokinetic chromatography (in normal CE neutral molecules will migrate through the capillary unseparated with a velocity equal to the EOF velocity), where a micellar pseudostationary phase is introduced in the separation electrolyte. More recently, capillary electrochromatography was introduced ${ }^{5}$ which combines the flat flow profile of $\mathrm{CE}$ with the hydrophobic separation capability of HPLC to enable mixed mode electrophoretic and reverse-phase chromatographic separations of both charged and neutral compounds. Size exclusion gels ${ }^{6}$ and polymer solutions ${ }^{7}$ have also been used to separate large analytes such as DNA fragments based on size/charge rather than mass/charge. Because of the excellent separation efficiencies that can be obtained using CE, it has evolved into a powerful and flexible separation technique for a diverse spectrum of analytes. $C E$ also has a number of other significant advantages over HPLC and GC:

- Requires only minute quantities of analyte.

- Instrumentation is simple and has few moving parts. 
- Inexpensive fused silica capillaries and minimal buffer reagent volumes result in very low cost of operation.

- Minute volumes simplify waste disposal problems.

Although CE has significant advantages over chromatographic techniques, it also has several limitations. Because of the narrow-bore capillaries, only minute quantities of analyte can be analyzed. Unlike HPLC, which can be scaled up to perform preparative separations, CE is limited to use as an analytical tool. Run-to-run precision of both response and migration time have been an issue for $\mathrm{CE}$, as peak shape and injected amount can be significantly effected by the sample matrix ${ }^{8}$. Thermal heating inside the capillary, resulting from the movement of ions, can lead to a parabolic flow profile due to a differential temperature (and hence differential viscosity) across the capillary. Although this Joule heating can decrease separation efficiency, it can usually be mitigated by using reasonable operating parameters (i.e. capillaries $<100 \mu \mathrm{m}$ i.d., buffers with moderate conductivity, separation voltages $<30 \mathrm{kV}$ ) or capillary cooling.

An aspect of $C E$ that has limited its general application is its lack of a simple, sensitive detection system. The most popular mode of detection employed in CE has been on-column UV detection. Although UV detection has provided good sensitivities for HPLC, in CE optical detection path lengths are reduced from several millimeters in HPLC to $<100 \mu \mathrm{m}$. Also, curvature of the capillary walls makes effective illumination of the inner capillary solution difficult and causes nonlinearity in Beer's Law because of the different path lengths through the analyte zone. A significant amount of effort has been spent trying to enhance the UV detector sensitivity using light-path length extending 
techniques such as bubble-cells, Z-cells and off-capillary detection ${ }^{9}$. Although these lightpath extending modifications can provide as much as a $\times 40$ gain in signal strength ${ }^{9}$, this is usually accompanied by an increase in the noise which reduces the ultimate $\mathrm{S} / \mathrm{N}$ increase. This extended path length can also decrease separation efficiency as this increased detection cell volume may extend over several theoretical plate heights. Due to their high cost, these extended-light path capillaries virtually eliminate one of the benefits CE has over techniques of HPLC - low consumables cost. Laser-induced fluorescence (LIF) has been used to achieve detection limits of $10^{-24}$ mole $^{10}$. This excellent detection sensitivity has enabled CE to be used for DNA fragment separations where fluorescent labels were already available from use in slab-gel electrophoresis. Unfortunately, chemicals of analytical interest do not normally fluoresce. In addition, the development of derivatizing regimes that would enable fluorescence detection while not affecting the CE separation would be difficult. This limited application is the most significant limitation of LIF. Smith et al used a mass spectrometer (MS) as a CE detector for attomole detection limits as well as for obtaining confirmatory identification of the analyte ${ }^{11}$, but due to the cost of the MS instrument and the difficulties encountered in the CE-MS interface, it is not currently considered a viable detection method for routine use.

Unlike most of the other detection techniques that have been used in $\mathrm{CE}$ which are mass-dependant (UV, fluorescence, MS), electrochemical detection (ECD) is a concentration dependant technique. Consequently, although the detectable mass of analyte is reduced, analyte concentration remains constant, and ECD should not suffer the same sensitivity losses when miniaturized. Amperometric detection has been used in 
HPLC to achieve sensitivities not possible with UV detection ${ }^{12}$. However, ECD has been relegated to a technique that is used only when necessary to achieve detection sensitivities not obtainable by other techniques. This is due partly to the fact that UV detection provides adequate detection sensitivity for a wide variety of analytes and is extremely easy to use, while HPLC-ECD is more selective and notoriously fickle to use on a routine basis due mainly to electrode fouling and baseline drifting (the composition of the mobile phase has a significant impact on the measured signal). Wallingford and Ewing first applied $E C D$ to $C E$ to analyze the easily oxidized catecholamines ${ }^{13}$. Although they were able to detect $20 \mathrm{fmol}$ amounts, complex apparatus was required to decouple the detection circuit from the high separation field. Since this initial publication, which introduced CE-ECD, over 250 publications have followed describing various applications of CE-ECD. Despite this interest, CE-ECD still suffers from the same poor reputation that exists for HPLCECD. While initial research papers for CE-LIF and CE-ECD were both reported in the late nineteen eighties, several commercial CE-LIF instruments have been available since 1995 , while the only commercially available ECD system for CE is a conductivity detector. This may indicate the lack of acceptance of ECD by the analytical community. The major reasons for the lack of interest in CE-ECD are as follows:

- Unlike optical detection schemes, the electrochemical probe is in intimate contact with the separation medium making it prone to contamination.

- Reported CE-ECD instrumentation is fragile and bulky (i.e. xyzmicropositioners and faraday cages used are quite large)- not suitable for routine use.

- Implications of measuring small currents in the high-separation field environment make instrumentation complex. 
- $\mathrm{CE}-\mathrm{ECD}$ is limited to analytes that are electrochemically active.

The purpose of this thesis was to address these limitations of current CE-ECD systems with the goal of developing a sensitive and widely applicable CE detection system suitable for routine diagnostic use. Initial work focused on the development of a squarewave voltammetry (SWV) ECD method for CE (Chapter 2). Early in the research program, though, it was determined that to develop a CE-ECD system attractive to practicing analytical chemists, simpler and more robust instrumentation was needed. Also, although commercial systems were available for performing SWV, none were capable of the high scan rates that would be demanded by CE. Chapter 1 describes the development and testing of a rugged and simpler CE-ECD system. Appendix A describes the development of a flexible computer-controlled data acquisition/control capable of investigating CE-SWV. The resulting CE-SWV system allowed the detection of electroactive neurotransmitters using significantly simpler and more robust instrumentation than had been previously reported with similar sensitivities. Although SWV provided excellent sensitivity for redox-type processes, one of the reasons for developing a CE-SWV system was to investigate whether it could be applied to detect other, more general electrode processes to develop a more generally applicable CE detection method. The AC nature of the SWV waveform allowed the possibility of monitoring other electrode-surface phenomena which change the double-layer structure, such as analyte adsorption. Although initial studies showed that physical adsorption of hydrophobic organic analytes produced an analytically significant SWV signal, further investigations revealed that using more extreme waveform potentials for analytes with $\pi$ - 
electron density also produced a significant SWV response that allowed excellent detection sensitivity for a wide range of organic analytes. This detection method, termed adsorption-based ECD (AdsECD), was optimized for its analytical application and further investigated by applying it to a wide variety of analytically important organic molecules. Development of these adsorption-based ECD techniques are described in Chapter 3. The development of AdsECD for CE coupled with the improvements made to the CE-ECD instrumentation resulted in a sensitive, robust and widely applicab le detection system for CE. 


\section{CHAPTER 1 - DEVELOPMENT OF ROBUST INSTRUMENTATION FOR CE-ECD}

\subsection{Introduction}

In the late nineteen eighties researchers were beginning to realize that although the resolving power of $C E$ was undisputed, the lack of a sensitive detection method would limit its acceptance as a useful analytical tool. ECD appeared to be a possible solution to this dilemma for electroactive analytes. Unlike mass-dependant detection techniques that suffer sensitivity losses when miniaturized, ECD, is primarily a concentration-dependant technique, and should maintain its sensitivity when miniaturized. Wallingford and Ewing demonstrated $^{13.14,15}$ that, while ECD did offer the potential of extremely good sensitivities for electroactive analytes, there were a number of technical problems that needed to be resolved before its application as a routine analytical tool could be realized. Using ECD instrumentation similar to that reported by Knecht et al for use in microbore $(15 \mu \mathrm{m})$ open tubular HPLC-ECD ${ }^{16}$, Wallingford and Ewing encountered what they considered a fundamental difficulty in interfacing ECD to CE - the effect that the high electric field created by the separation potential has on the measurement of small faradic currents. The basic instrumental setup reported by Knecht et al (microbore HPLC) was a system for positioning a $\mu \mathrm{m}$-scale carbon fiber cylindrical electrode into the end of the separation capillary for use in amperometric detection mode. In CE, ECD is complicated due to the electric field which exists along the length of the separation capillary. A cylindrical 
carbon fiber electrode inserted into the separation capillary, as proposed by Knecht et al, would experience a potential drop along its length. With typical CE separation fields of $500 \mathrm{~V} / \mathrm{s}$, a potential gradient of $5 \mathrm{~V}$ would exist along a $100 \mu \mathrm{m}$ carbon fiber inserted into the separation capillary. Thus attempts to maintain a uniform working electrode potential would not be possible. To avoid this potential gradient, Wallingford and Ewing developed an off-column detection scheme that isolated the working electrode from the separation field. A second, short piece of capillary was coupled to the separation capillary using a section of porous glass. This joint was then immersed in a buffer with a cathode and the detection electrode was inserted into the end of the short capillary. In this configuration, the ionic current was conducted through the porous joint, and the EOF pushed the separation capillary contents through the short detection capillary. Zone broadening due to pressure-driven flow in the detection capillary was minimized by use of a short detection capillary. Wallingford and Ewing also found that using capillaries with smaller inner diameters increased the sensitivity of electrochemical detection due to a reduced layer thickness between the capillary wall and cylindrical electrode surface which, they theorized, promoted a higher coulometric efficiency ${ }^{15,17}$. Using this off-capillary ECD method, detection limits of $20 \mathrm{fmol}$ were reported ${ }^{15}$.

A number of alternate methods have also been developed to decouple the electrophoretic current from the detection system. Huang and Zare ${ }^{18}$ created a porous glass frit in the wall of a fused silica capillary by boring a hole in the capillary with a $\mathrm{CO}_{2}$ laser and heat-sintering solder glass in the hole. This frit was then immersed in a buffer with the terminating high-voltage electrode, and detection was accomplished at the end of 
the capillary, after the frit. $\mathrm{O}^{\prime}$ 'Shea et $\mathrm{al}^{19}$ created a ion-conductive capillary joint by scoring a capillary, covering it with Nafion ${ }^{\circledR}$ tubing secured with epoxy then bending the capillary slightly to create a fracture. Kok et al ${ }^{20}$ joined two capillaries with a Pd coupler. The Pd was able to pass enough electrolytically generated hydrogen to support the electrophoretic current. Park and Lunte cast a highly conductive perfluorosulfonated ionomer detection cell onto the end of a fused silica capillary ${ }^{21}$. Although this detection cell had the same inner diameter as the capillary, because it was conductive, no potential drop existed inside the joint, and electrochemical detection could be performed inside it. $\mathrm{Hu}$ et al etched the outside of a small section of a fused silica separation capillary such that its reduced wall diameter permitted ionic conduction ${ }^{22}$.

The purpose of all of these various means of creating an ion-conductive section in the separation capillary was to allow cylindrical detection electrodes (most commonly carbon fiber) to be inserted into the capillary. The major advantage in using cylindrical carbon fiber electrodes was the high coulometric efficiencies that could be achieved. Knecht et al' ${ }^{16}$ initial work describing the use of cylindrical carbon fiber electrodes reported efficiencies near 100\% (at typical open tubular HPLC flow rates). However, there are a number of significant limitations that accompany this detection scheme. These conductive unions are both fragile and difficult to construct. Because all of the reported conductive unions are constructed as part of the separation capillary, any fouling, breakage or plugging of the capillary would require a new conductive union to be fabricated. Also, cylindrical carbon fiber electrodes are fragile and can not be reconditioned by mechanical polishing. This is a rather severe limitation that would require new electrodes to be 
prepared frequently. Furthermore, these off-capillary detection systems work only when a reasonably high EOF exists to push the capillary contents through the detection capillary. For low-pH electrolytes where the EOF is low to nonexistent, off-capillary detection can not be used. These limitations prevent this type of detection system from being used on a routine basis.

Sloss and Ewing ${ }^{23}$ reported a method which allowed a cylindrical carbon fiber electrode to be used without a decoupling system. By HF etching the end of 5 and $2 \mu \mathrm{m}$ capillaries to produce a conical opening, the resistance (and potential drop) at the capillary end was reduced, allowing a carbon fiber to be inserted into it without using a decoupling device.

Huang et al reported a different approach to improving CE-ECD. They used an end-capillary detection system employing a carbon fiber disk-electrode positioned near the end of a $5 \mu \mathrm{m}$ separation capillary $y^{24}$. Although the detection limits they obtained were an order of magnitude higher than had been reported using cylindrical carbon-fiber electrodes with decoupling apparatus, their approach was significantly simpler. Unlike a cylindrical electrode inserted in the capillary, a disk electrode aligned with the end of the capillary is normal to the field produced by the separation voltage. Although the potential of the electrode will be shifted due to the separation field, the potential across the face of the electrode should remain constant.

Although the CE-ECD apparatus reported by Sloss and Ewing (conical opening etched in the detection-end of the capillary) as well as Huang et $\mathrm{al}^{24}$ (carbon-fiber disk 
electrode) were simpler than those requiring decoupling systems, they both used narrow bore capillaries $(2-5 \mu \mathrm{m})$ which are troublesome to use as they become blocked easily. Also, the CE-ECD system reported by Sloss and Ewing et al still used cylindrical carbon fiber electrodes.

Lu et al used both $\mathrm{Hg}$-film disk electrodes and cylindrical carbon fiber electrodes in end-capillary detection mode with $25 \mu \mathrm{m}$ separation capillaries to detect metal ions (Hg-film electrode) and catechol (carbon-fiber electrode) without using a field decoupler ${ }^{25}$. Detection limits obtained for catechol compared favorably with those obtained using off-capillary detection methods. By carefully examining the impact of electrode positioning (axial and capillary-electrode distance/insertion depth) on $\mathrm{S} / \mathrm{N}$ in this high-field environment they were able to obtain detection limits for catechol that compared favorably with those obtained using off-capillary detection methods (i.e. using field decouplers ${ }^{15}$. Chen and Huang reported an end-capillary ECD cell that used a 50 $\mu \mathrm{m}$ Pt disk electrode and $5 \mu \mathrm{m}$ separation capillary $y^{26}$. By using end-capillary detection they avoided the complexity of a decoupling device, but the reported construction procedure for the working electrode and ECD cell was complex resulting in an assembly that would be no more rugged than the decoupling devices mentioned earlier. Although detection limits of 23-66 $\mathrm{nM}$ for several neurotransmitters were reported using this apparatus, these were not consistent with electropherograms included in the report, where approximate $\mathrm{S} / \mathrm{N}$ estimates from an electropherogram of a $25 \mu \mathrm{M}$ standard mixture shown indicate that detection limits at least an order of magnitude higher may be more realistic. Fermier et al used end-capillary detection with a $300 \mu \mathrm{m}$ carbon electrode and $13 \mu \mathrm{m}$ 
separation capillary ${ }^{27}$. This large diameter electrode did not require exact alignment of the electrode with the end of the capillary, and a relatively simple ECD cell was used. Unfortunately, the reported detection limits of $4 \mu \mathrm{M}$ for neurotransmitters were several orders of magnitude higher than those that had been obtained using carbon fibers and decoupling devices. Matysik reported an improved disk-electrode, end-capillary detection method for $\mathrm{CE}$ for use with capillaries of 25 and $50 \mu \mathrm{m}$ inner diameter ${ }^{28}$. Using a $30 \mu \mathrm{m}$ carbon fiber disk-electrode, detection limits approximately an order of magnitude higher than those obtained using off-capillary detection ${ }^{15}$ were obtained. In this report, Matysik noted the influence of the separation fieid on the potential of the working electrode positioned close to the exit of the capillary. After the magnitude of the potential shift was determined by observing the shift in the half-wave potential of an electroactive analyte added to the electrolyte, this offset potential was added to the potential applied to the working electrode to correctly set its potential when the separation potential was applied.

The design of most CE-ECD systems reported with adequate sensitivities precludes incorporation into a commercial automated $C E$ unit $^{13-17,19,24}$. These systems usually employ an XYZ-micropositioner to position the electrode in the detection zone. Also, faraday cages are typically used to provide shielding from environmental noise. The faraday cage is commonly a box made from metal screen that encloses the entire CE-ECD system. A compact CE-ECD cell that allows sensitive detection while allowing easy replacement/realignment of the capillary and electrode has not yet been reported. There have been relatively simple CE-ECD cells reported that use mm-scale electrodes ${ }^{27.29,30}$ in a wall-jet configuration, but mm-scale electrodes usually result in poorer sensitivities than 
can be achieved using $\mu \mathrm{m}$-scale electrodes. Also, using mm-scale electrodes precludes the use of rapid voltammetric techniques as charging currents for such large-scale electrodes would be prohibitively high. This chapter will report the development of CE-ECD instrumentation that addresses these shortcomings of systems previously reported.

Based upon the review of CE-ECD techniques that have been reported, it appeared that development of a robust CE-ECD system using cylindrical electrodes inserted into the capillary would be difficult due to the complexity of the decoupling systems required and the fragility of the electrode. Disk $\mu \mathrm{m}$-scale electrodes are relatively easy to construct and avoid the necessity of decoupling systems, but have generally resulted in poorer sensitivities. Early work investigating square-wave voltammetry detection (SWV) for CE (see Chapter 2) indicated that sensitivities comparable to those obtained using amperometric detection with high-coulometric efficiency carbon fiber electrodes and decoupling systems were possible using a much simpler end-capillary disk electrode system. During the development of the CE-SWV technique, though, it became evident that to produce a reliable $\mathrm{CE}$ detection system for routine use, robust $\mathrm{CE}-\mathrm{ECD}$ instrumentation was needed. The requirement that the CE-ECD system must be suitable for routine use places a number of constraints on the instrumentation. Firstly, the cell must be capable of maintaining the capillary-electrode axial alignment and distance for extended periods without requiring frequent re-alignment. Typical vibrations encountered in a laboratory environment must not affect the cell alignment or produce noise in the detector output. Because rapid-scanning voltammetric techniques would be investigated using this cell, it needed to be capable of using $\mu \mathrm{m}$-scale electrodes (as opposed to using 
larger scale electrodes to eliminate the necessity for fine alignment adjustments). The cell should also permit either capillary or electrode removal and replacement easily. Finally, because diagnostic application of CE-ECD will require an automated CE system to be used, the CE-ECD cell should be compact enough to allow the possibility of retrofitting a commercial CE system to accommodate CE-ECD.

These requirements eliminated a number of previous CE-ECD cell designs, particularly those using decoupling devices and carbon fiber electrodes (lacking robustness, difficult to change electrode/capillary) and those that used 3-D micropositioners. Also, the detection sensitivities which appeared possible using CESWV permitted the inherently more robust end-capillary, disk-electrode detection mode to be used. Because end-capillary detection does not require a separation-field decoupling system, much of the complexity and fragility of previously reported sensitive CE-ECD systems could be eliminated. To eliminate the bulky 3-dimensional micropositioners that had been employed by others to align $\mu \mathrm{m}$-scale electrodes with the capillary, a small, rugged CE-ECD cell was developed that allowed relatively easy adjustment of both the axial alignment and distance between the capillary and electrode as well as removal and re-insertion of the separation capillary cell without requiring re-adjustment of the capillary-electrode axial alignment. In addition, a robust electrode system was developed to complement this CE-ECD cell. Metal wire $\mu \mathrm{m}$-scale electrodes were fabricated using glass capillaries sheathed with a stainless-steel sleeve. This sleeve gave the electrode mechanical stability, was the terminating electrode for the high-voltage separation power supply, acted as the auxiliary electrode for the potentiostat used to control the potential of 
the working electrode, and provided shielding from environmental noise. The resulting CE-ECD system was compact enough to be accommodated by most commercial CE systems and its ruggedness and easy alignment made it suitable for routine use. Having developed the CE-ECD cell, the effect of electrode position at the end of the separation capillary for this CE-ECD cell was investigated to determine not only the optimal electrode position but also the magnitude of the CE-SWV response and electropherogram peak shape changes that result from capillary-electrode misalignment. The latter was done to investigate the general reliability of the device and the precision with which alignment and distance adjustments must be made, which are important considerations when considering a technique for routine use. These capillary-electrode alignment and distance studies lead to an improved technique to adjust the electrode position within the detection zone. It used the shift that is observed in potentials applied to a working electrode placed in the separation electric field rather than the tradition optical verification of electrode placement. The effect of the inner diameter of the separation capillary on detection sensitivity was also investigated. Finally, to verify the general stability and reliability of the reported CE-ECD instrumentation, the repeatability (injection-to-injection, after capillary-electrode realignment and day-to-day) of the system was investigated.

\subsection{Experimental}

\subsubsection{Chemicals}

All solutions were prepared from water purified using an ULTROpure with a TFM membrane and NANOpure II with Type 1 ORGANICfree cartridge kit (Bamstead Corp., Dubuque, LA). Unless otherwise stated, all reagents were analytical grade. 
Sulfamethazine, sulfapyridine, sulfadimethoxine and sulfathiazole were obtained from Sigma-Aldrich Canada Limited (Oakville, ON, Canada). N-(2-heptyl)-Nmethylpropargylamine (2-HMP) and Deprenyl were obtained from the Department of Neuropsychiatric Research, University of Saskatchewan (Saskatoon, SK).

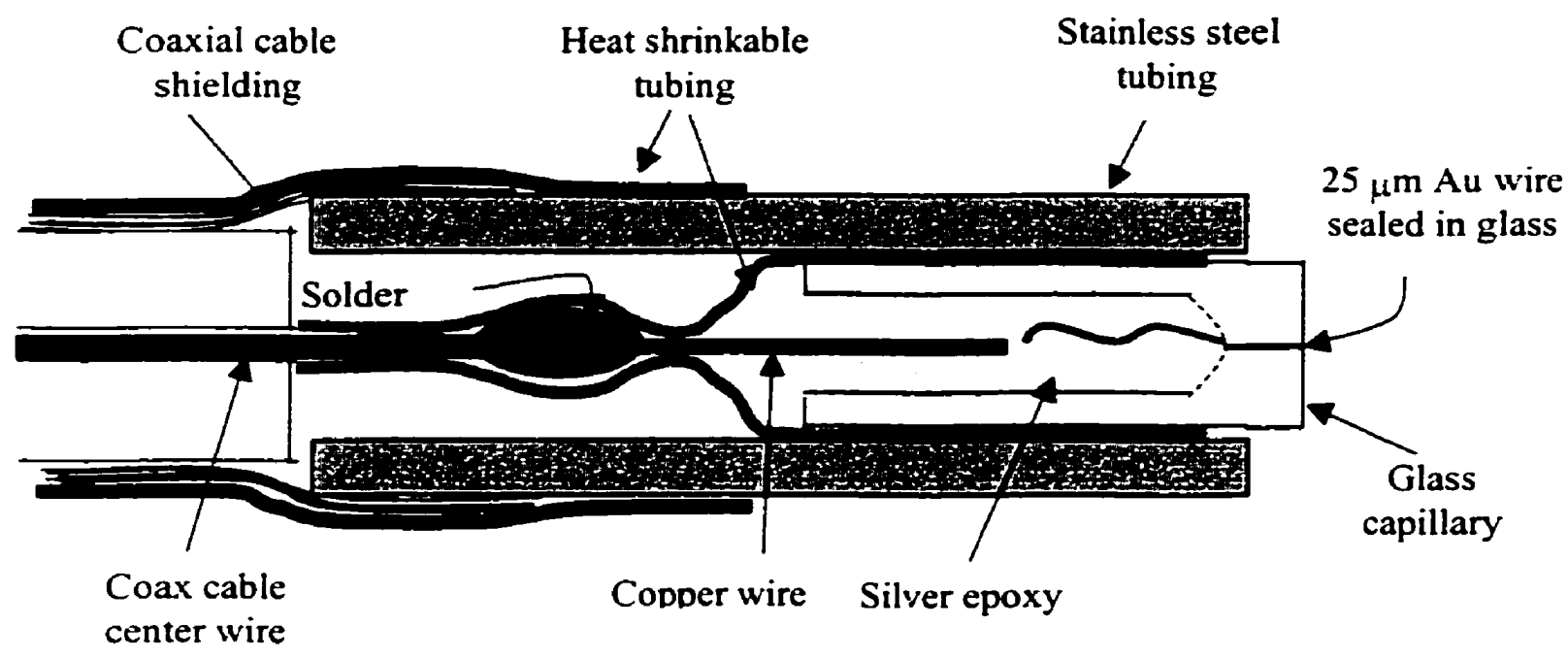

Figure 1.1 - Working/auxiliary electrode assembly schematic.

\subsection{2 um-Electrodes}

The electrodes used for electrochemical detection, shown schematically in Figure 1.1 , were prepared by sealing a $25 \mu \mathrm{m}$ gold, platinum or palladium wire (Goodfellow, Berwyn, PA, U.S.A.) in a $10 \mu \mathrm{l}$ micropipette tube (Canadawide Scientific, Ottawa, ON, Canada). Only about 1-2 mm of the end of the capillary tube was melted around the wire using a short exposure to a butane flame. The micropipette tube was cut to a length of $\sim 2$ $\mathrm{cm}$, silver epoxy was tamped into the open end, and a $0.5 \mathrm{~mm}$ copper wire was slid into the capillary to make contact with the $25 \mu \mathrm{m}$ electrode wire. The copper wire was soldered to the core wire of a shielded cable and heat-shrinkable tubing was used to cover 
the glass electrode and exposed wire. A $3 \mathrm{~cm}$ length of $1 / 8$ " o.d. $\times 1 / 16$ "i.d. stainlesssteel tubing, filled with standard 5 min epoxy, was slid over the electrode assembly, allowing the sealed electrode to extend out $\sim 5 \mathrm{~mm}$ from the end of the stainless-steel tube. Excess epoxy was wiped away and this assembly was allowed to cure for $1 \mathrm{~h}$. Contact was made between the stainless-steel tubing and the coaxial cable shielding by extending the shielding over the stainless-steel tubing and securing it with heat-shrinkable tubing. The glass electrode was ground using wetted 3200 grit Micro-mesh ${ }^{2}$ abrasive paper (Micro-mesh, Wilton, Iowa, U.S.A) to expose the $25 \mu \mathrm{m}$ disk followed by further polishing with wetted 6000 grit Micro-mesh ${ }^{\circledR}$ abrasive paper to provide a smooth electrode surface. This electrode was then secured in the CE-ECD cell shown in Figure 1.2 using a nut and ferrule. The stainless-steel provided adequate shielding of the working electrode as well as acting as the auxiliary electrode in the potentiostat and terminating electrode for the high-voltage power supply.

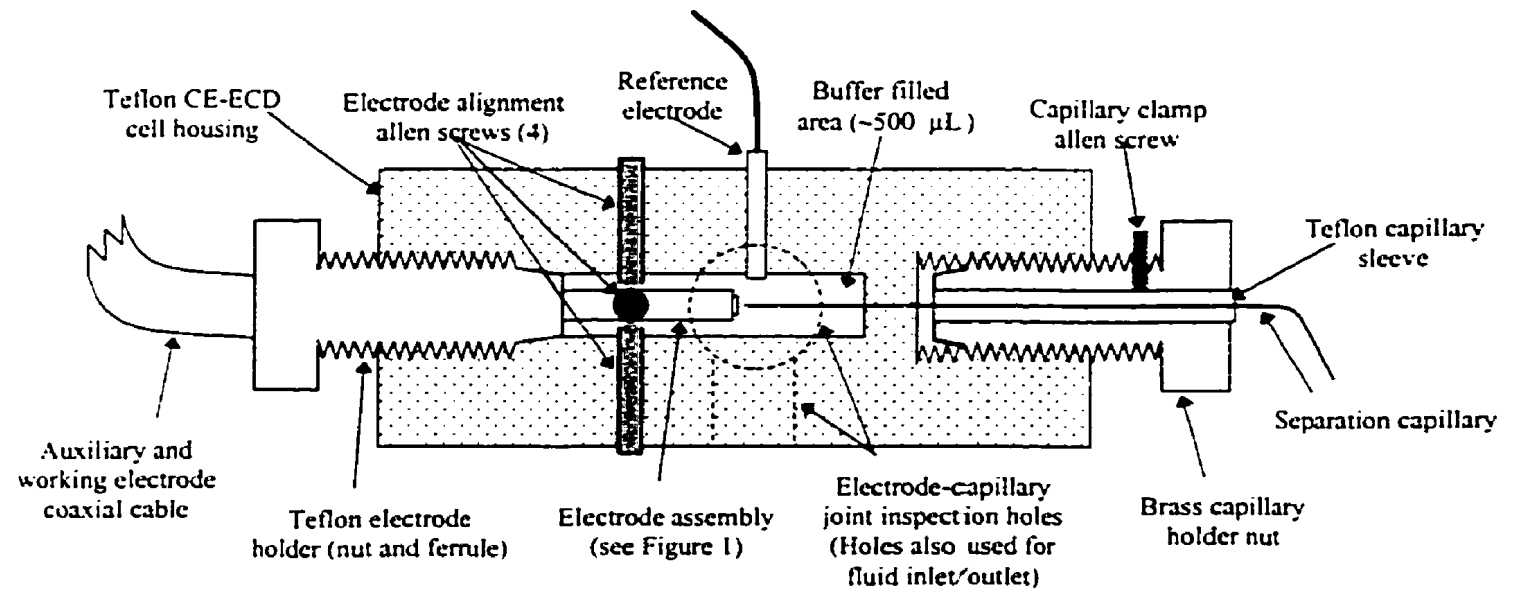

Figure $1.2-$ CE-ECD cell schematic. 


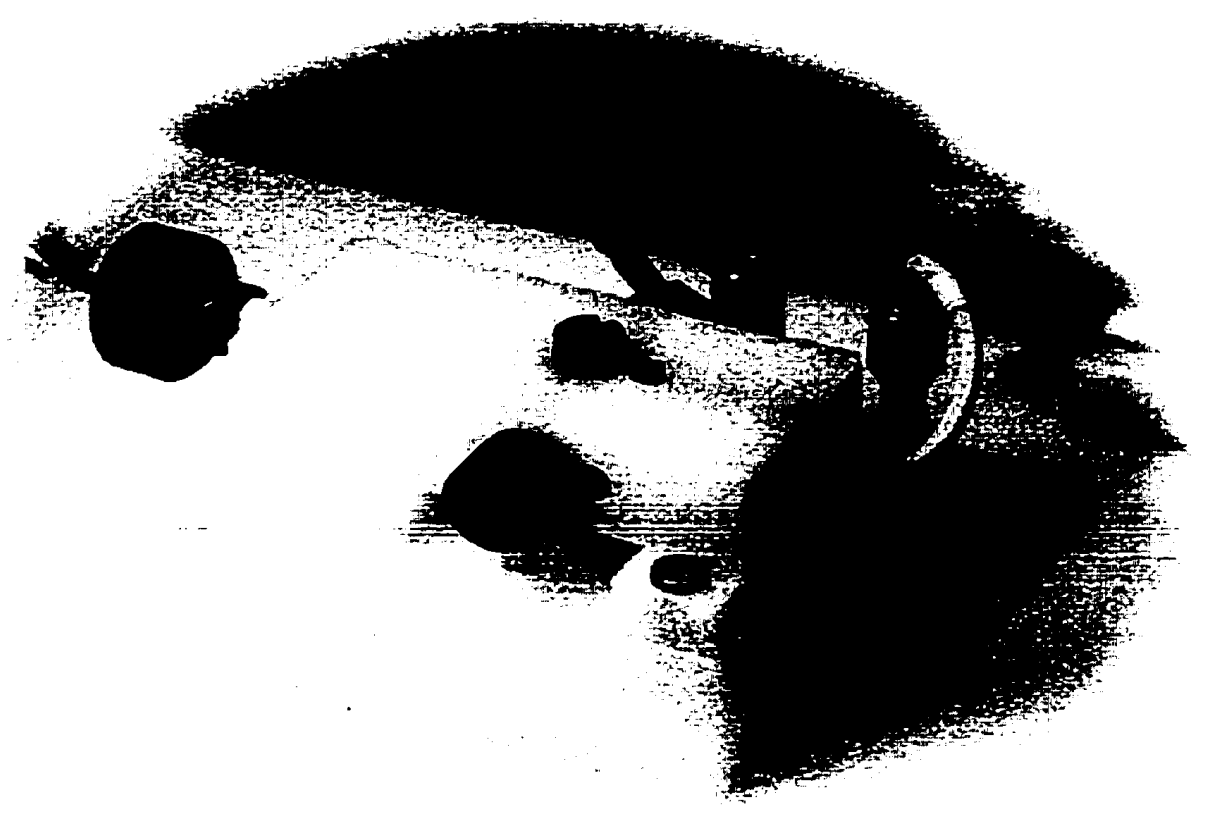

Figure 1.3 - Picture of CE-ECD cell.

\subsubsection{CE-ECD cell}

The schematic of the electrochemical cell that was used for end-column electrochemical detection in CE is shown in Figure 1.2 (a constructed cell is shown in Figure 1.3). Prototypes of the cell were constructed from both Teflon and Delrin. Once the capillary and electrodes were fastened in the cell, axial alignment was achieved by adjusting the four allen screws around the electrode. Although axial alignment was initially verified optically with a x20 Hastings triplet magnifier (Edmond Scientific, Barrington, NJ), an alternate measure of axial alignment will be discussed in the Results and Discussion section. A capillary was inserted through the capillary holder nut until it came in contact with the face of the electrode. It was then secured in place by tightening 
the allen screw on the capillary holder nut. A thread pitch of $\mathbf{4 0}$ threads-per-inch was used for the capillary holder nut which resulted in a distance adjustment scale of $1.8 \mu \mathrm{m} / \mathrm{degree}$ turn of the nut. Although this adjustment mechanism provided a stable and precise means of adjusting and maintaining the capillary-electrode distance, accurate, absolute distance measurements were difficult. For studies of the effect of capillary-electrode distance, a scale was mounted around the nut that allowed its degree of rotation to be measured to an accuracy of $\sim \pm 2{ }^{\circ}$. Once the electrode and capillary were axially aligned, the capillary could be removed and replaced routinely requiring only re-establishment of the optimal capillary-electrode distance. Holes in the side of the CE-ECD cell accommodated a miniature $\mathrm{Ag} \mid \mathrm{AgCl}$ reference electrode (Model EP1, World Precision Instruments, Sarasota, FL) and fluid connections. All waveform potentials given in this report are versus this reference electrode. The electrolyte in the cell was automatically replenished using a peristaltic pump that was started whenever the capillary was rinsed after each run.

\subsubsection{Potentiostat and current amplifier}

The electronic circuit diagram of the potentiostat and current amplifier used is shown in Figure 1.4. All resistors (metal film) had an error of $\pm 1 \%$ and the amplifiers were LF353LN wide-bandwidth dual-JFET operational amplifiers (Cardinal Electronics, SK, Canada). The electronic circuit was enclosed in a metal box. Shielded coaxial cables were used for connections to the auxiliary/working electrode assembly and reference electrode. 

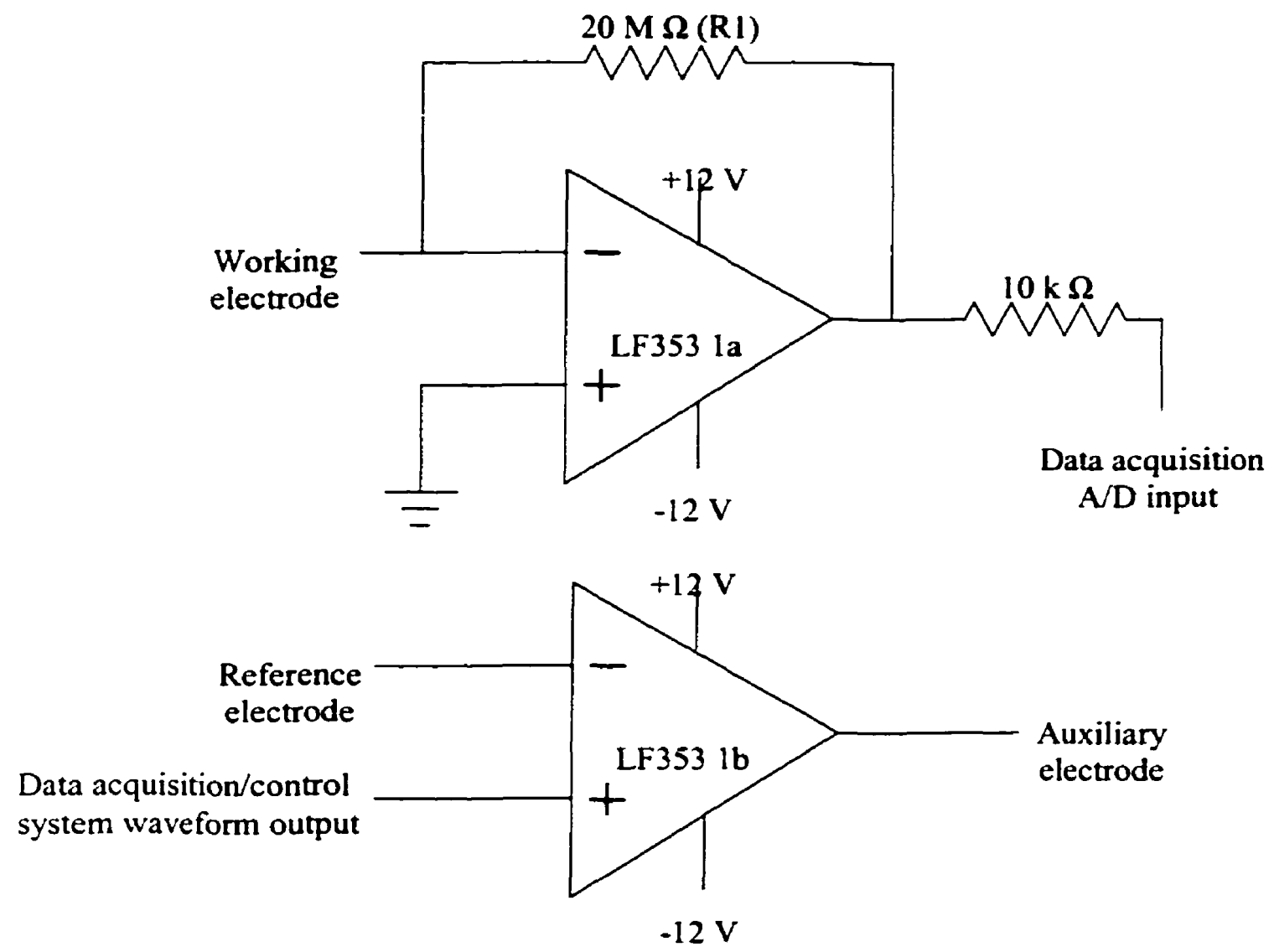

Figure 1.4 - Potentiostat and current amplifier schematic.

\subsubsection{Data acquisition system}

The data acquisition system used for these experiments is described in Appendix

A. 


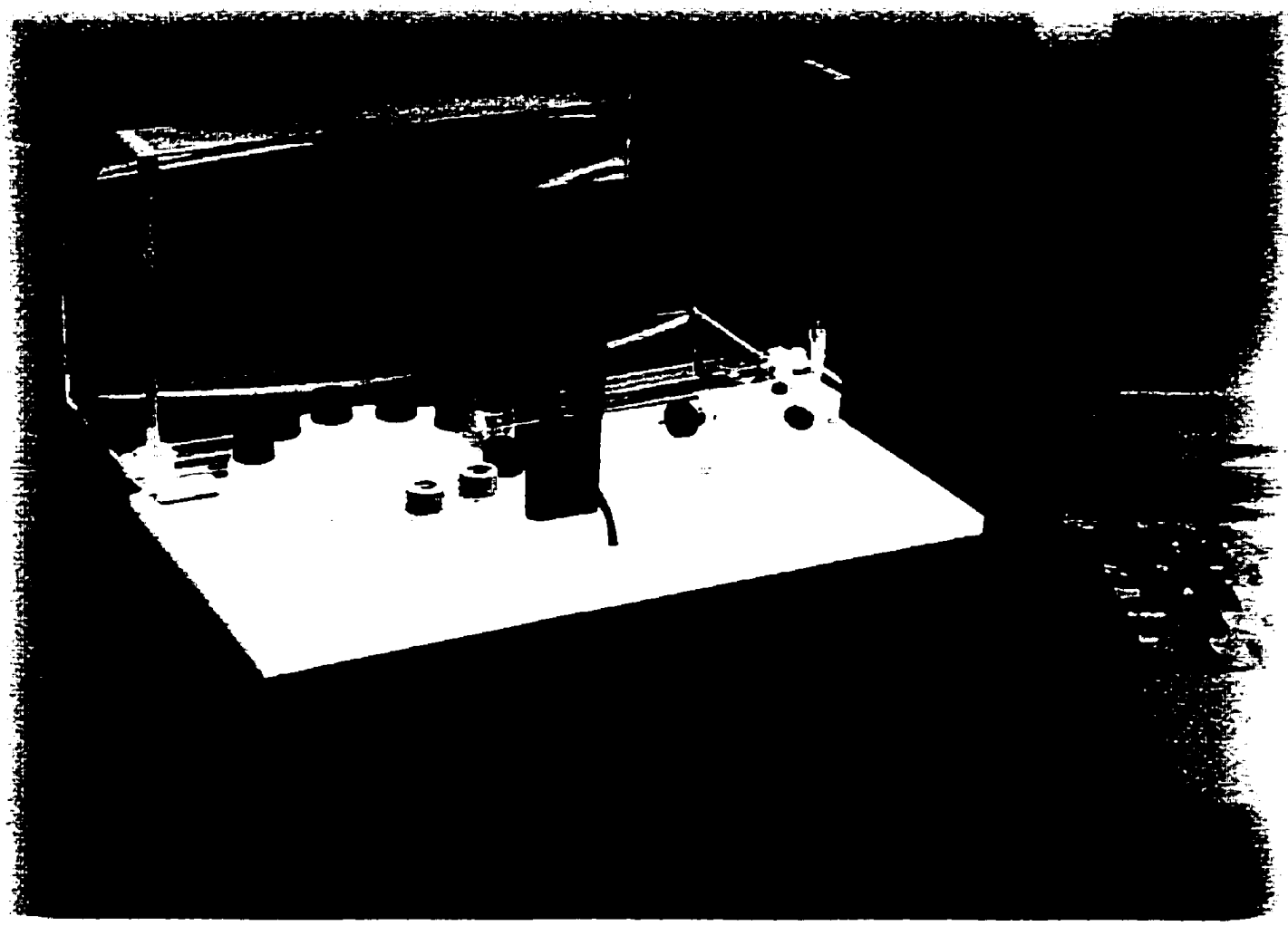

Figure 1.5 - Picture of in-house constructed automated CE-ECD system.

\subsubsection{Automated CE system}

A computer-controlled CE was constructed to inject samples, pressure-rinse capillaries and perform separations automatically (see Figure 1.5). The autosampler consisted of a circular sample tray controlled by a stepper motor; the tray accommodated $12,10 \mathrm{~mm} \times 32 \mathrm{~mm}$ glass vials. Viais were lifted and pressure sealed to the capillary/electrode assembly by a stepper-motor controlled elevator. Opto-electronic devices were used to sense the position of both the elevator and autosampler tray. The CE separation voltage was supplied by a Spellman MM1OPN $10 \mathrm{kV}$ power supply (Spellman High Voltage Electronics Corporation, Plainview, NY, USA). The entire CE system (tray 
and lifter motors, pressure source, high-voltage power supply) was controlled with a 16C73 PIC microcontroller (Microchip Technology Inc., Chandler, AZ, U.S.A.). This microcontroller also provided data acquisition/control to perform SWV (see Appendix A). Software written with the programming language Delphi 4.0 (Borland, Scotts Valley, CA, USA), controlled the automated CE and was capable of performing programmed sequences of capillary rinses, injections and separations. Changes to the electrochemical detection method could also be made incrementally which allowed automated optimization of the detection parameters from a series of replicate injections.

\subsubsection{CE conditions}

Fused-silica capillaries (10, 25 and $50 \mu \mathrm{m}$ i.d., $365 \mu \mathrm{m}$ o.d., Polymicro Technology, Phoenix, AZ) were used in these experiments. Before use each day, capillaries were rinsed with $0.1 \mathrm{M}$ potassium hydroxide for $5 \mathrm{~min}$ followed by the separation electrolyte for $5 \mathrm{~min}$. Also, before each injection, the capillary and CE-ECD cell were rinsed with $\sim 2-5$ void volumes of the separation electrolyte. All sample injections were made electrokinetically. Unless otherwise stated, samples were diluted to the desired concentration using the CE separation electrolyte. The instances in this thesis where "sample stacking" (electrokinetic injection where the sample is prepared in an electrolyte of significantly lower ionic strength than the separation electrolyte which can result in a much more concentrated sample plug injected onto the capillary), was used will be clearly indicated.

Two systems were used to investigate the performance of the CE-ECD systems that were developed. These were sulfonamide antibiotics, separated using a $100 \mathrm{mM}, \mathrm{pH}$ 
7.0 phosphate electrolyte and Deprenyl and 2-HMP, separated using a $20 \mathrm{mM}$ $\mathrm{H}_{3} \mathrm{PO}_{4} / \mathrm{NaH}_{2} \mathrm{PO}_{4}$ buffer (1:1) adjusted to $\mathrm{pH} 3.0$ with triethylamine. Both systems used a $25 \mu \mathrm{m} \times 30 \mathrm{~cm}$ capillary and $10 \mathrm{kV}$ separation potential. The AdsECD method described in Chapter 3 was the electrochemical detection method used. The AdsECD method uses square-wave voltammetry to monitor changes occurring to the working electrode as analytes exit the capillary and interact with it. Analyte adsorption and possibly decomposition/reduction appear to be responsible for the response observed. The following SWV waveform and data acquisition parameters were used for both systems: $\mathrm{DC}$ voltage ramp : -600 to $-1100 \mathrm{mV}$ in $100 \mathrm{~ms}$; Square-wave frequency/amplitude: 1000 $\mathrm{Hz} / 100 \mathrm{mV} ; 0 \%$ of initial square-wave current response rejected. For a more complete discussion of the CE-AdsECD of these analytes, the reader is referred to Chapter 3.

\subsubsection{Calculations of $S / N$ and peak efficiency and asymmetry}

The $\mathrm{S} / \mathrm{N}$ ratio was determined by calculating the standard deviation $\left(\sigma_{n-1}\right)$ of a representative section of the electropherogram baseline ( -10 peak widths) near the analyte peak and dividing this into the height of the analyte peak. Peak efficiency was calculated as the number of theoretical plates $(N)$, where $N=5.54\left(t_{R} / W_{1 / 2}\right)^{2}, t_{R}$ is the migration time and $W_{1 / 2}$ is the peak width at half height. Peak asymmetry was calculated as peak front area/ back area (peak front/back determined by the dividing the peak at its apex) at $10 \%$ peak height. All $\mathrm{S} / \mathrm{N}$ and peak efficiency data presented here were averages of values obtained from at least three consecutive CE injections performed using similar conditions. Injection-to-injection precision of this instrumentation is discussed in Section 1.3.6. 


\subsection{Results and discussion}

\subsubsection{CE-ECD cell and $\mu m$-scale electrode}

The major challenge in developing a CE-ECD cell suitable for end-capillary

detection using $\mu \mathrm{m}$-scale electrodes was achieving capillary-electrode axial alignment. Initially, work in the development of a compact CE-ECD cell focused on using glass and plastic tubing to align the capillary and electrode. The general approach was to use a tubing sleeve to hold both the capillary and electrode in a concentrically aligned position. For this type of cell, it was best if the electrode and capillary had identical outer diameters. Electrodes were prepared by threading $25 \mu \mathrm{m}$ electrode wire into a $50 \mu \mathrm{m}$ i.d. fused-silica capillary and sealing with epoxy. To expose the electrode surface, the epoxy from the end of the capillary had to be removed using abrasive paper. This proved difficult due to the fragility of the capillary. Also, because a $25 \mu \mathrm{m}$ wire was threaded into a $50 \mu \mathrm{m}$ capillary, a concentrically aligned electrode was very difficult to achieve. Alternatively, electrodes were prepared in larger diameter micropipette capillary tubes $(\sim 1.2-1.8 \mathrm{~mm}$ o.d. and $\sim 400-$ $800 \mu \mathrm{m}$ i.d.) as described in the experimental section except without the stainless-steel shielding. Although the resulting electrode diameter was larger than that of the separation capillary, Teflon tubing was fitted over the separation capillary to bring its o.d. similar to that of the electrode. A "V" shaped groove was filed in the middle of a $2 \mathrm{~cm}$ piece of glass tubing with an i.d. similar to the o.d of the electrode and Teflon capillary sleeve. The electrode and capillary were fitted into this sleeve until they met at the filed "V," and both were secured in place using heat-shrinkable tubing. Although this cell was compact and reasonably rugged, it was difficult to set the electrode capillary distance precisely. Also, it was difficult to construct electrodes in which the electrode wire is sealed in the 
center of the glass tubing. Only one in $\sim 5-10$ attempts could produce a concentrically positioned electrode wire. As a result, a cell design with an adjustable electrode-capillary axial-alignment was constructed. Initially, a Peek HPLC “T”' (Upchurch Scientific, Oak Harbor, WA) was used for the CE-ECD cell. This "T" was drilled to accommodate the capillary and electrode, which were held in the in-line fittings of the "T." Electrode and capillary alignment screws were tapped into the sides of the "T." The third hole in the "T" was used for rinsing the cell. A hole was drilled in the side of the " $T$ " to observe the electrode-capillary alignment and hold a micro reference electrode. By adjusting the alignment screws while observing the electrode-capillary joint through the inspection holes good axial alignment could be achieved. The capillary was threaded through a glass micropipette tube which was held in the "T" using a standard HPLC fitting. Using heat, a small bend was made in the micropipette tube which provided enough friction to hold the capillary stationary while also allowing it to be manipulated to adjust the capillaryelectrode distance.

Although the cell was reasonably rugged and provided adjustment of the axial alignment and capillary-electrode distance, it had several problems. It was difficult to seal the cell properly to prevent it from leaking. Also, the walls of the " $T$ " were thin because it had been drilled out to accommodate the electrode, which resulted in poor threading of the fine screws used for alignment. The internal volume of the cell was also extremely limited $(\sim 20 \mu \mathrm{L})$ which can cause depletion of the buffer during electrophoresis. However, because the general concept of this cell appeared good, this design approach was used as a basis for a fabricated CE-ECD cell with more appropriate proportions as described in the 
experimental section and shown in Figure 1.2. The fabricated CE-ECD cell was more compact than the three-dimensional micropositioners that have been used by other workers to align the capillary and electrode and provided good mechanical stability for the capillary-electrode union. Prototypes of the cell were constructed from both Teflon and Delrin. Although Teflon had better chemical stability than Delrin, Delrin was more rigid which made tapping and drilling small holes easier. For aqueous-based electrolytes, no degradation of Delrin cells was observed as long as they were rinsed well with water before storage. Initial prototypes were prepared from $10 \mathrm{~mm}$ x $10 \mathrm{~mm}$ x $45 \mathrm{~mm}$ blocks which resulted in a cell volume of $\sim 200 \mu \mathrm{l}$. Subsequently, cells were prepared from 10 $\mathrm{mm} \times 20 \mathrm{~mm} \times 45 \mathrm{~mm}$ blocks to enlarge the internal volume to $\sim 500 \mu \mathrm{L}$. The larger volume reduced depletion of the electrolyte and provided better diluted of the byproducts of the electrophoresis reaction that were suspected to migrate past the working electrode and into the capillary to cause baseline drifting.

The CE-ECD cell system was also improved by the addition of a stainless steel sheath to the working electrode (Figure 1.1). This sheath was connected to the shielding on the coaxial cable connecting the electrode to the potentiostat. It was used both as an auxiliary electrode for the potentiostat as well as the pseudo-ground electrode for the highvoltage power supply. It is described as a pseudo-ground for the high-voltage power supply since, as shown in Figure 1.4, it was not connected to ground, but set at the waveform potential - typically -2 to $+2 \mathrm{~V}$. These potentials relative to typical separation potentials of several kilovolts, provided adequate grounding. Because it was a good 
conductor that surrounded the working electrode, it also provided shielding of external electromagnetic radiation.

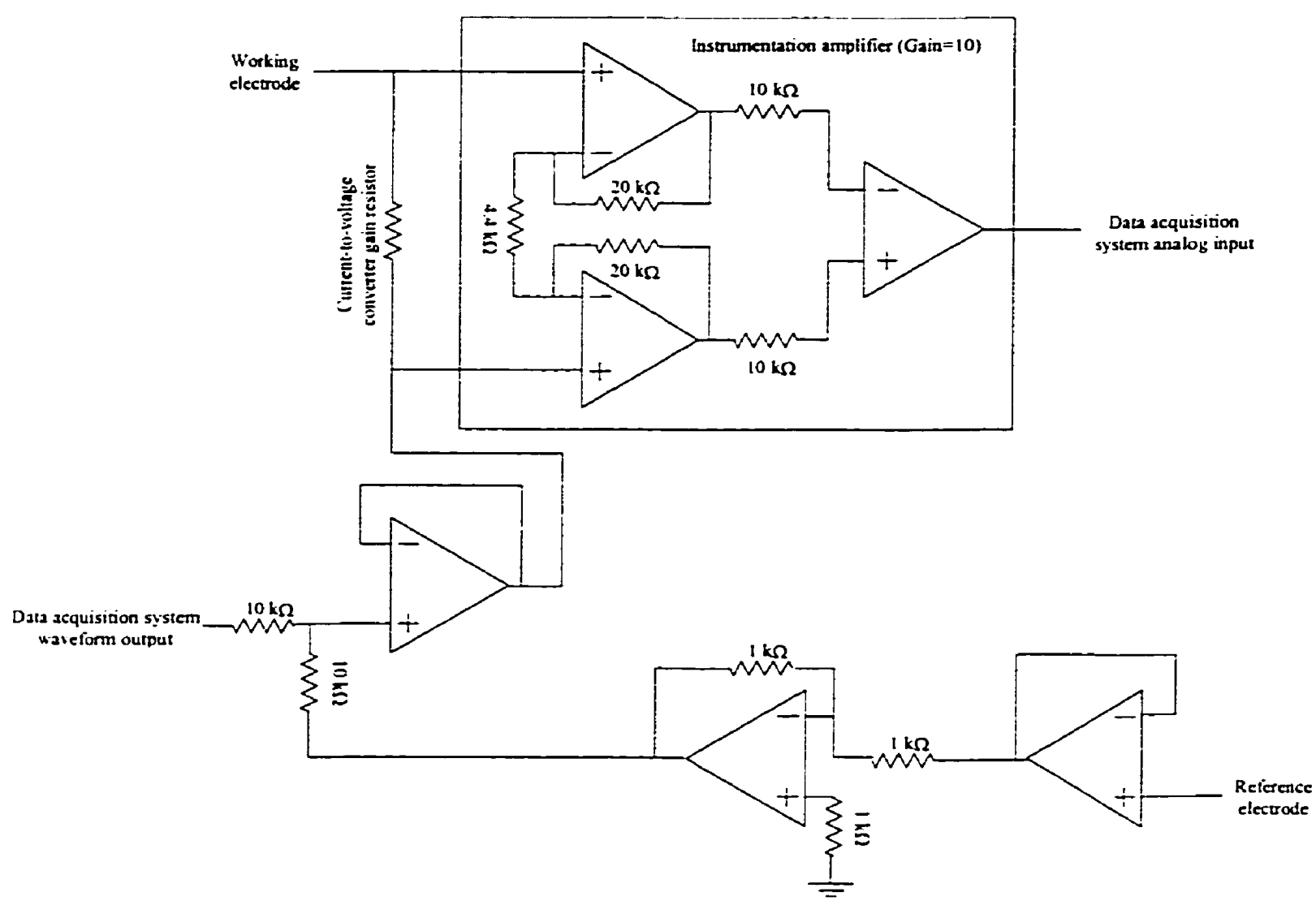

Figure 1.6 - Schematic of instrument amplifier-type potentiostat

\subsubsection{Potentiostat circuits}

Two potentiostat circuits were examined for use in rapid scanning voltammetric detection. Figure 1.6 illustrates the basic design of the initial potentiostat circuit used which was similar to that used by $\mathrm{Lu}$ et $\mathrm{al}^{25}$. In this circuit the potential of the working electrode was applied through the gain resistor via a summing/inverting amplifier that combined the reference electrode potential and waveform potential. The potential drop across the gain resistor measured by the instrumentation amplifier was proportional to the 
current flowing through the working electrode. Although this potentiostat design was used in initial testing, closer examination of this circuit revealed that it could limit the application of rapid scanning ECD techniques. In the latter the potential of the working electrode changes rapidly, and its resulting current response is measured. This situation could result in high, short lived charging currents and the potential of the working electrode would be attenuated by the high currents flowing through the gain resistor (i.e., a current of $100 \mathrm{nA}$ generated at the working electrode would result in a voltage drop of 100 $\mathrm{mV}$ across a $1 \mathrm{M} \Omega$ gain resistor). To avoid this problem, an alternate potentiostat design was investigated (Figure 1.4). In this potentiostat the potential of the electrolyte is adjusted via the auxiliary electrode potential. Deviations in the auxiliary-solution potential are accommodated for by the reference electrode feedback. The current flowing through the working electrode is measured using an operational amplifier configured as a currentto-voltage converter/amplifier. In this potentiostat design, current flowing through the working electrode should not effect its potential (providing the auxiliary electrode is large enough to prevent its polarization). It should also be noted that in this potentiostat design, the working electrode is held at virtual ground (the op-amp used as a current follower will attempt to maintain equality of its two inputs via the feedback resistor, thus keeping the potential of working electrode connected to the inverting input equal to the grounded noninverting input). Potential of the solution-working electrode interface is controlled by applying an inverted potential to the auxiliary electrode (i.e., if an working electrode potential of $+1 \mathrm{~V}$ is desired, $-1 \mathrm{~V}$ is applied to the auxiliary electrode via the potentiostat). The schematic illustrated in Figure 1.4 is only a functional description of the circuit used. 
The complete circuit that was used, which included programmable gain, offset adjustment and output limiting features to accommodate the digital-to-analog converters used in the data acquisition system, is described in Appendix $\mathbf{A}$.

Control of the potential of the working electrode using a potentiostat system also presented difficulties with interfacing the CE-ECD system to commercial CE instruments. Although the fabricated CE-ECD cell was of suitable dimensions to be accommodated by the automated Beckman P/ACE 5500 commercial CE instrument (Beckman Coulter, Fullerton, CA), complications arose retrofitting this instrument for ECD detection. This instrument, as well as most commercial CE instruments, grounded the terminating electrode in the power supply through a resistor. The potential drop across this resistor is measured to determine the electrophoretic current through the capillary during a separation. Because the CE-ECD potentiostat design shown in Figure 1.4 controls the potential of this terminating electrode, measurement of the electrophoretic current is distorted. In the Beckman P/ACE 5500 instrument a safety feature stops the high voltage power supply when the electrophoretic current measured at the inlet and outlet of the capillaries is substantially different, which would normally indicate a potentially dangerous ground leak. This feature prevented the CE-ECD potentiostat that was developed from being used on the Beckman instrument. Because an automated system was desired to thoroughly examine CE-ECD, the automated CE system described in Section 1.2.6 was developed specifically for use with the CE-ECD system. 

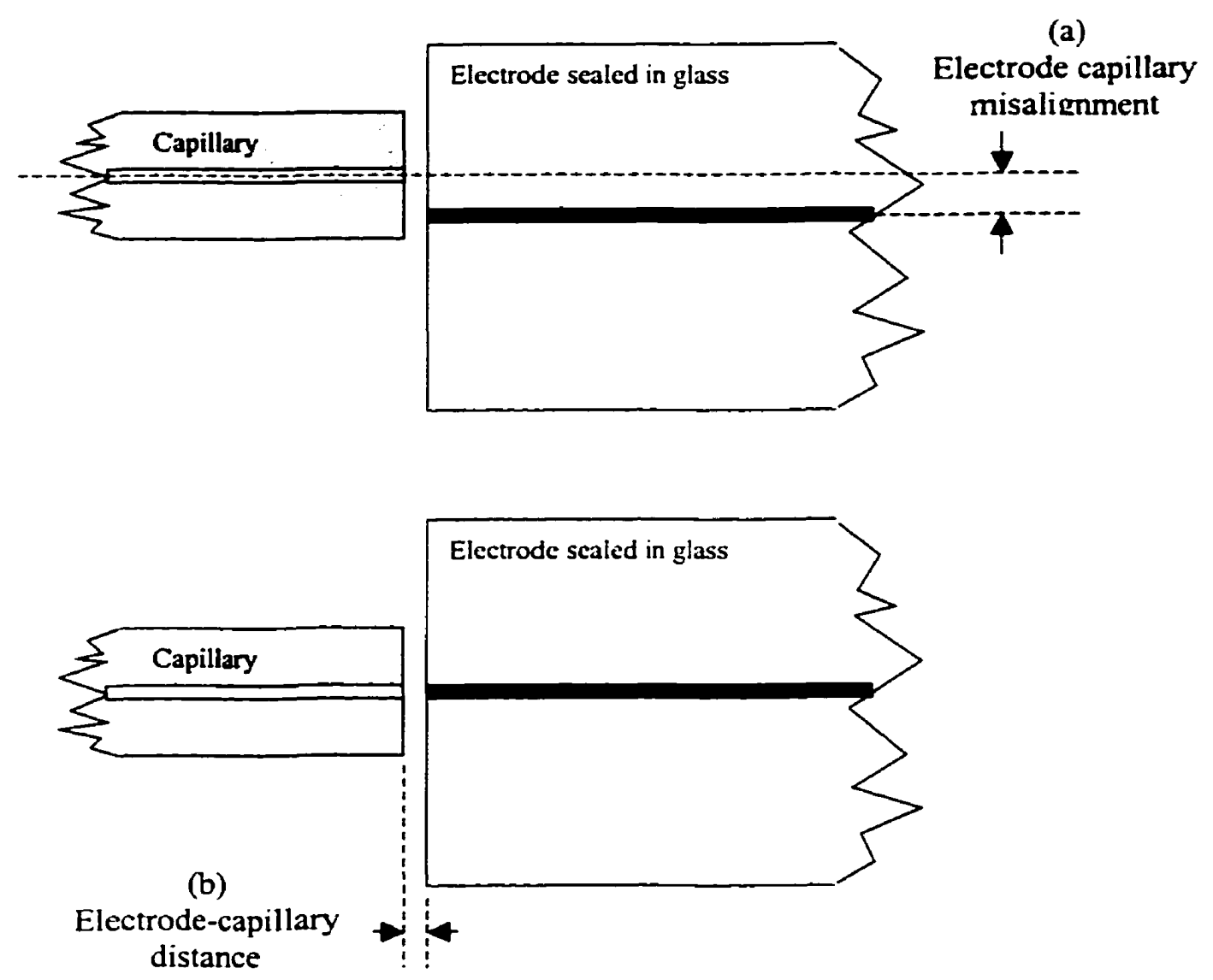

Figure 1.7 - Illustration of capillary-electrode misalignment (a) and distance (b).

\subsubsection{Effect of capillary-electrode distance and axial alignment}

In end-capillary ECD with a $\mu \mathrm{m}$-scale disk, the axial alignment of the capillary and electrode as well as the distance between the capillary and electrode will impact several aspects of the CE-ECD system. Lu et $\mathrm{al}^{25}$ illustrated the importance of capillaryelectrode axial alignment and distance in CE-ECD using disk-electrodes. To determine the effect the precision of capillary-electrode axial alignment and distance had on electrode response, similar studies were performed using the CE-ECD system described 
above. The diagram shown in Figure 1.7 illustrates, for the purposes of this discussion, what is meant by capillary-electrode distance and axial alignment/misalignment.

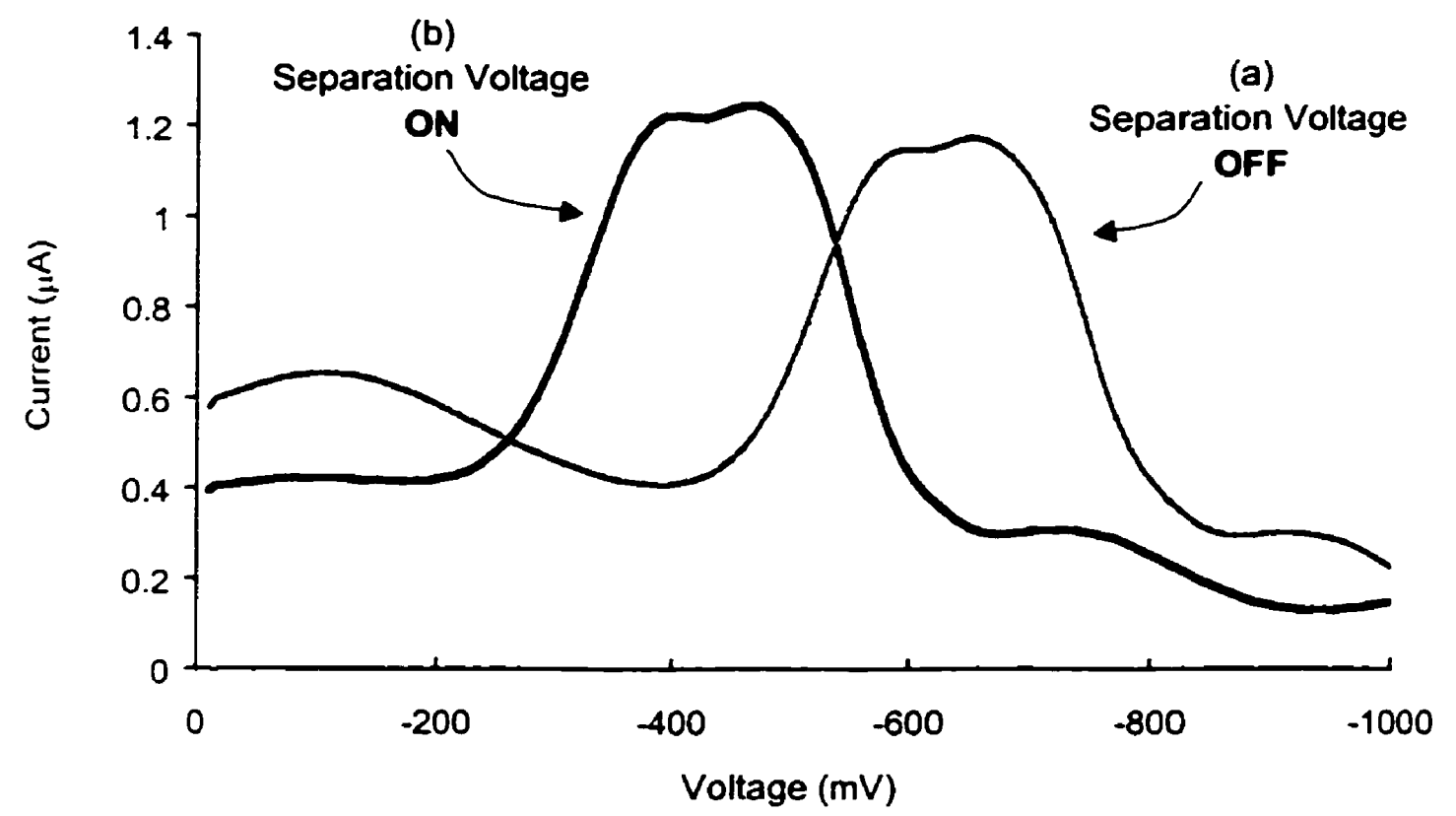

Figure 1.8 - Voltammogram of background response observed for a Pt electrode in $100 \mathrm{mM}, \mathrm{pH} 7.0$ phosphate buffer (A - hydrodynamic conditions: B $-10 \mathrm{kV}$ separation voltage on). SWV conditions : 0 to $-1000 \mathrm{mV} / 100 \mathrm{~ms} \mathrm{DC} \mathrm{ramp}, 1000 \mathrm{~Hz}, 100 \mathrm{mV}$ square-wave, $30 \mathrm{~ms} / 1000 \mathrm{mV}$ post-scan pulse, $30 \mathrm{~ms} / 0$ $\mathrm{mV}$ pre-scan pulse.

SWV was used to quantitate the effect of the adjustments made (the reader is referred to Chapter 3 which describes application of SWV to CE). Because SWV is a scanning technique that shows the dependence of the electrode current response on applied potential, it can measure how the potential field from the separation voltage affects the electrode response. SWV scan potentials can usually be found for an electrolyte that reveals a potential-dependant current response, usually due to oxidation of the electrode material or adsorption/reduction of the electrolyte. By scanning a potential region in which a potential-dependant current response is observed, the impact of capillary- 
electrode alignment and distance can be examined. Figure 1.8 illustrates the background response observed for a Pt electrode in $100 \mathrm{mM}, \mathrm{pH} 7.0$ phosphate buffer obtained under hydrodynamic conditions (i.e., while pressure rinsing the capillary with electrolyte) with the electrode axially aligned and $\sim 30 \mu \mathrm{m}$ away from a $25 \mu \mathrm{m} \times 30 \mathrm{~cm}$ separation capillary (Figure 1.8, curve a). The current response observed in this negative potential region is likely due to the adsorption/reduction of hydrogen onto the Pt electrode. When a $10 \mathrm{kV}$ separation voltage was applied across the separation capillary, the current response of the Pt electrode was shifted by $\sim 200 \mathrm{mV}$ (Figure 1.8 , curve b). This potential shift was due to the electric field generated by the separation potential. The magnitude of the potential shift will be determined by the $i \mathrm{R}$ drop ( $i$ - electrophoretic current, $\mathrm{R}$ - solution resistance between the working and reference electrodes) that exists between the reference and working electrode. Although the function of the reference electrode is to sense changes in the solution potential and adjust the output of the auxiliary electrode accordingly, due to spatial restrictions the reference electrode cannot be positioned close to the working electrode to sense the changes in the solution potential at the working electrode's position due to the separation electric field. As a result, the potential of the solution at the working electrode's position is shifted from the desired applied potential. Both the capillaryelectrode axial alignment and distance will change the solution resistance between the reference working electrode, and hence affect the magnitude of the potential shift that occurs when the separation potential is applied. The effect of capillary-electrode axial alignment on the potential shift at the working electrode is illustrated in Figure 1.9. 


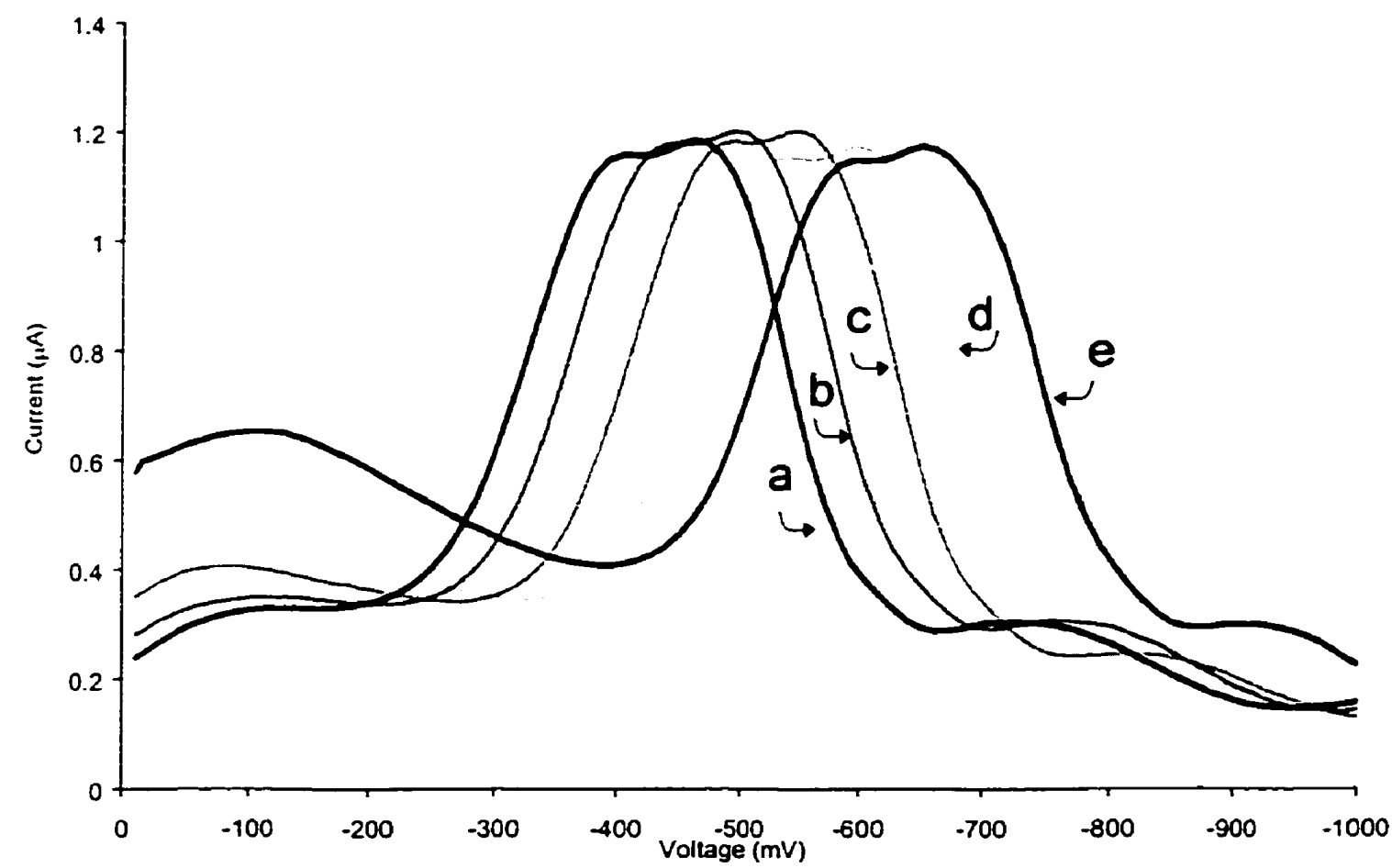

Figure 1.9 - Potential shift observed when a $10 \mathrm{kV}$ separation voltage was applied and the capillary and electrode were intentionally misaligned by $0 \mu \mathrm{m}$ (a), $13 \mu \mathrm{m}$ (b). $90 \mu \mathrm{m}$ (c), $180 \mu \mathrm{m}$ (d). Curve (e) indicates the SWV response observed when the separation voltage turned off. See Figure 1.8 for SWV and electrolyte conditions.

To obtain perfect capillary-electrode axial alignment the axial adjustment screws were manipulated until the $25 \mu \mathrm{m}$ capillary inner hole was perfectly aligned with the 25 $\mu \mathrm{m}$ exposed disk of the electrode. The capillary-electrode misalignments shown in Figure 1.9 were achieved by aligning the electrode with the outside edge of the capillary inner hole (13 $\mu \mathrm{m}$ misalignment), halfway between the outside edge of the capillary inner hole and the outside of the capillary ( $90 \mu \mathrm{m}$ misalignment) and finally aligning the electrode with the outside edge of the capillary (i.e. polyimide coating, $180 \mu \mathrm{m}$ misalignment). The error in these capillary-electrode misalignments was estimated to be $\sim 10 \%$. The capillaryelectrode distance (Figure 1.7b) was maintained at $30 \mu \mathrm{m}$ throughout this axial-alignment 
study. When the electrode was perfectly aligned with the capillary, where the resistance between it and the reference electrode was greatest, the maximum potential shift was observed [i.e., Figure 1.9, curve (a) versus curve (e)]. As the electrode was increasingly misaligned, the amount of potential shift decreased [Figure 1.9, curves (b)-(d) versus curve (e)].

Figure 1.10 illustrates the effect that capillary-electrode distance (Figure 1.7b) had on potential shift. When the capillary-electrode distance was small (i.e. maximum resistance, hence maximum $i R$ drop, between the working and reference electrode), a large potential shift was observed (Figure 1.10, curve a). As the capillary was moved away from the electrode, the potential shift was reduced (Figure 1.10, curves b-f). To apply the correct potential to the working electrode, once the working electrode was positioned, the potential shift that was observed with the separation voltage turned on was recorded and added to the waveform applied to the working electrode during subsequent separations. Figure 1.10 also shows the effect that small capillary-electrode distances had on the square-wave voltammetry. When the electrode was positioned very close to the capillary (Figure 1.10, curve a), it appears that the ohmic drop resulting from excessive solution resistance broadened and lowered the SWV peak. These are typical symptoms of excessive ohmic drop, and illustrates the problems that would be encountered if one attempted to use SWV with fiber electrodes inserted inside capillaries (off-capillary mode). 


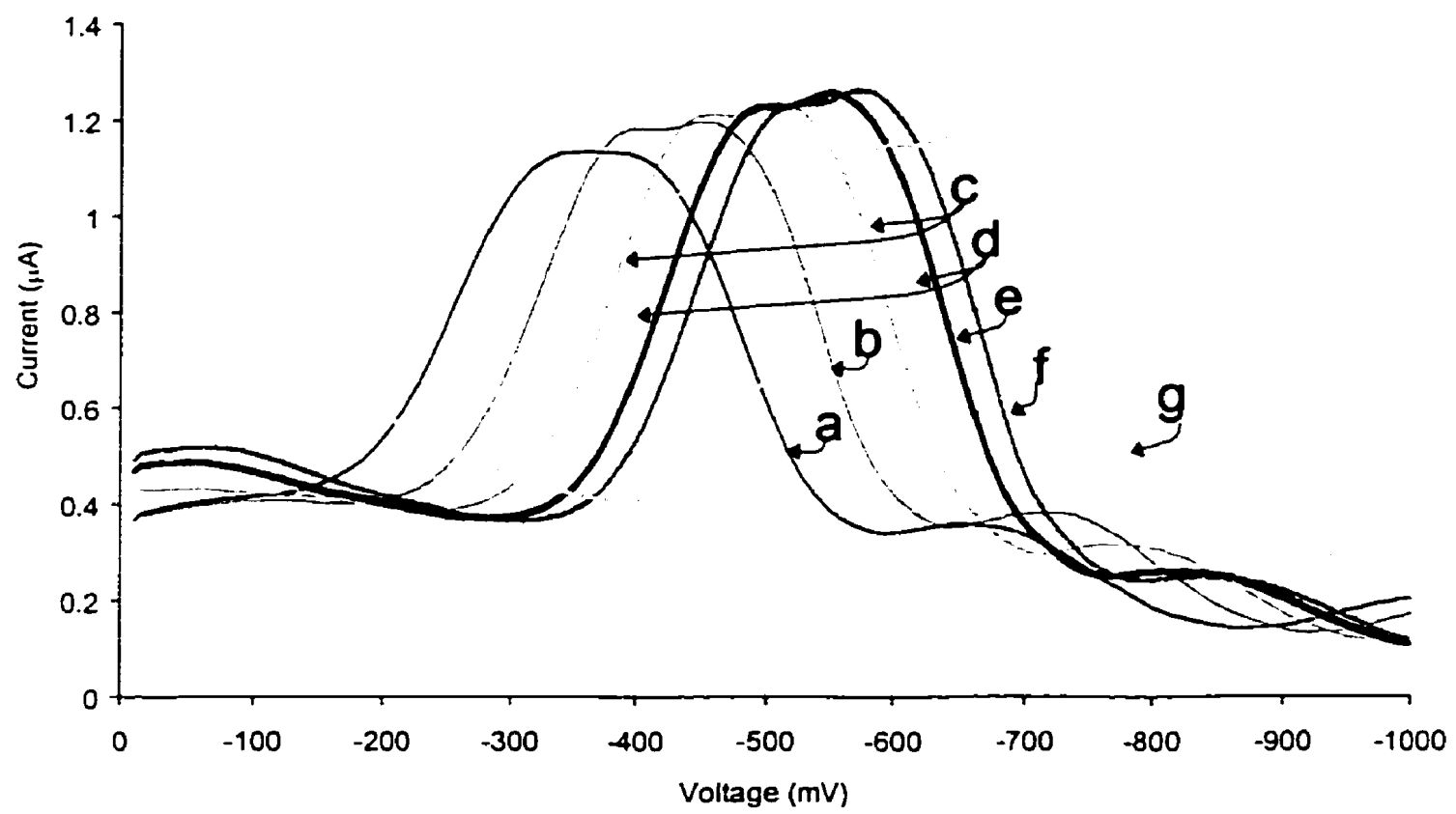

Figure 1.10 - Effect of capillary-electrode distance on potential shift observed. Curves represent the SWV voltammograms observed when a $10 \mathrm{kV}$ separation potential was applied and the capillary-electrode distance was adjusted to $150 \mu \mathrm{m}$ (f), $80 \mu \mathrm{m}$ (e), $50 \mu \mathrm{m}$ (d), $30 \mu \mathrm{m}$ (d), $20 \mu \mathrm{m}$ (b) and $<5 \mu \mathrm{m}$ (a). Curve (g) was observed when the separation potential was turned off. See Figure 1.8 for SWV and electrolyte conditions.

The effect of capillary-electrode alignment and distance on the sensitivity and peak shape was examined. For these experiments adsorption-based electrochemical detection (AdsECD) was used as the detection method (Chapter 3 describes the application of CEAdsECD). AdsECD was used primarily because it has a wider application than other electrochemical methods. Analytes detectable using AdsECD were available as probes at both low and high $\mathrm{pH}$. Figure 1.11 illustrates the effect that capillary-electrode distance (Figure 1.7b) had on the peak shape of trimethoprim, using a pH 7.0 separation electrolyte (the capillary and electrode were axially aligned). As the distance between the capillary and electrode was increased, the peaks decreased in height, increased in width, and began to tail. Increasing the capillary-electrode distance will increase, the volume available for 
the analyte to diffuse into during the detection process. This diffusion will dilute the analyte, reducing its signal (peak height). Also, the increased detection volume results in more "separation zones" being incorporated in each detector scan, effectively increasing the time constant of the detector. This has the effect of broadening sample zones that existed as narrow bands in the capillary.

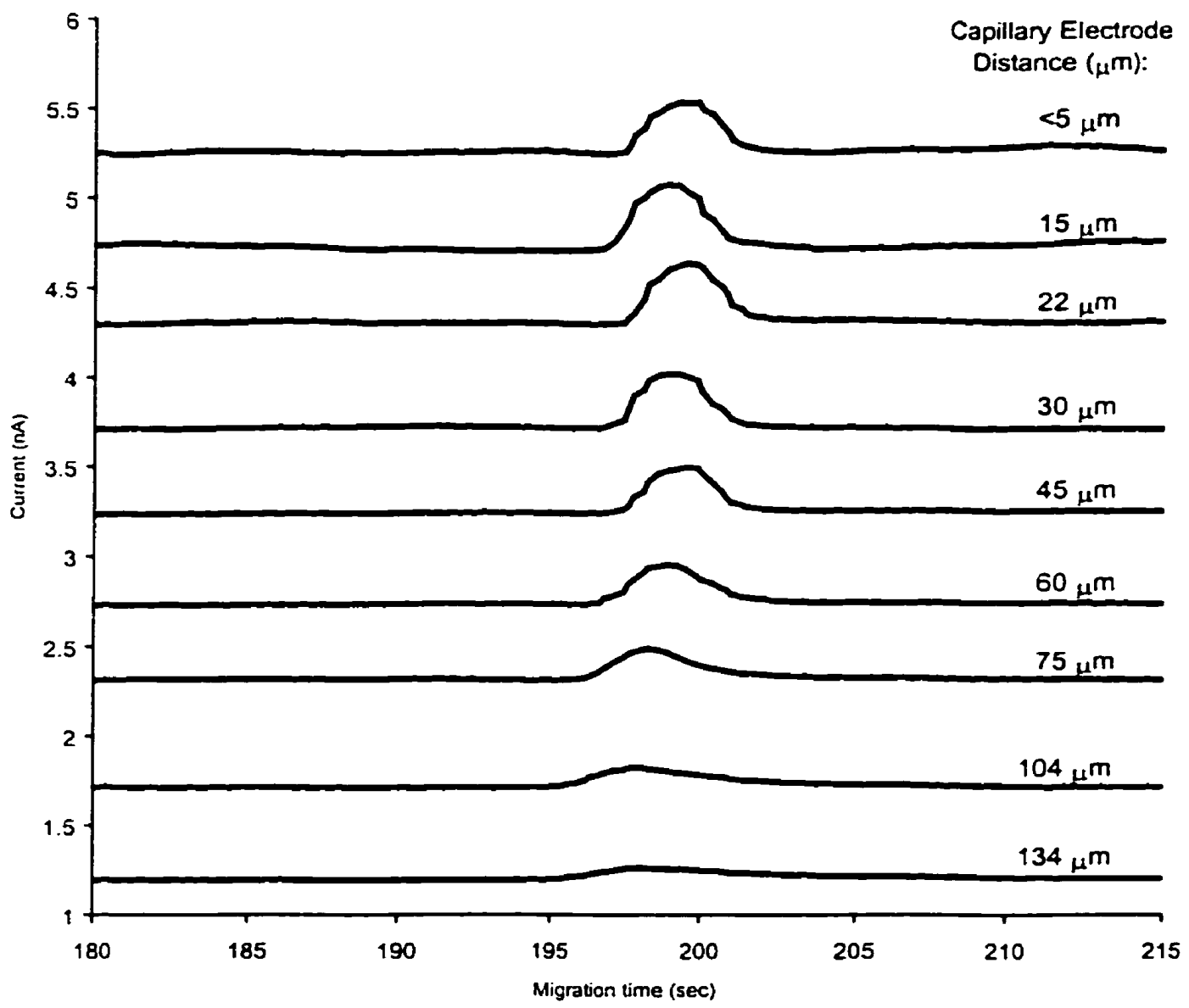

Figure 1.11 - Effect of capillary-electrode distance on trimethoprim peak shape using a pH 7 separation electrolyte. CE conditions: $\mathrm{pH} 7.0,100 \mathrm{mM}$ phosphate buffer, $25 \mu \mathrm{m} \times 30 \mathrm{~cm}$ capillary, $10 \mathrm{kV}$ separation voltage. $1 \mu \mathrm{M}$ trimethoprim injected electrokinetically for $3 \mathrm{~s} @ 10 \mathrm{kV}$. 


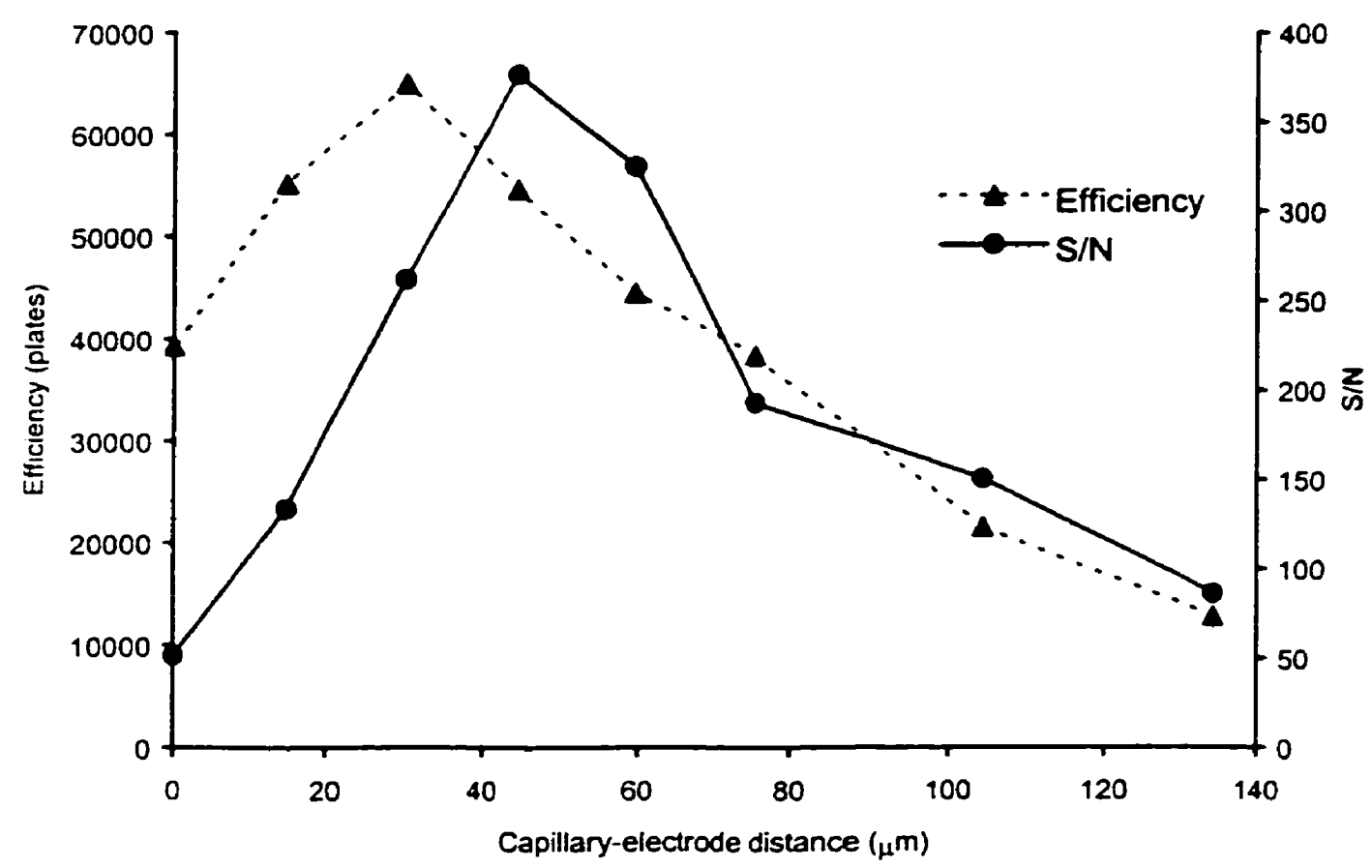

Figure 1.12 - Effect of capillary-electrode distance on separation efficiency and $\mathrm{S} / \mathrm{N}$ for trimethoprim. See Figure 1.11 for conditions.

Figure 1.12 illustrates quantitatively the effect capillary-electrode distance had on both separation efficiency and $\mathrm{S} / \mathrm{N}$ for the electropherograms in Figure 1.11. As was observed in Figure 1.11 , the signal strength and peak efficiency increase as the capillary is brought closer to the electrode. When baseline noise is considered, $\mathrm{S} / \mathrm{N}$ begins to decline when capillary-electrode distances $<40 \mu \mathrm{m}$ were used. This decline in signal strength may be due to the increased $i \mathrm{R}$ drop as the film of electrolyte between the working electrode and bulk solution is narrowed. Although the potential shift caused by this $i \mathrm{R}$ drop was compensated for, as discussed above, by adding an offset voltage to the applied waveform, the capacity of the electrode to react to the rapidly changing potentials that exist in the SWV waveform is diminished by the increased $i \mathrm{R}$ drop. The distortion of the 
voltammogram peak at close capillary-electrode distances illustrated in Figure 1.10, curve a, indicates that ohmic drop effects were observed. It is also possible that at capillaryelectrode distances less than $30 \mu \mathrm{m}$ the diffusion zone of the electrode is encroached upon which ultimately will reduce its coulometric efficiency. It was rather unexpected to observe the peak efficiency decrease when the capillary was brought close to the electrode. To better understand the nature of this peak efficiency decrease, the effect of capillary-electrode distance on peak asymmetry (a good measure of peak tailing) for these same separations was investigated (Figure 1.13).

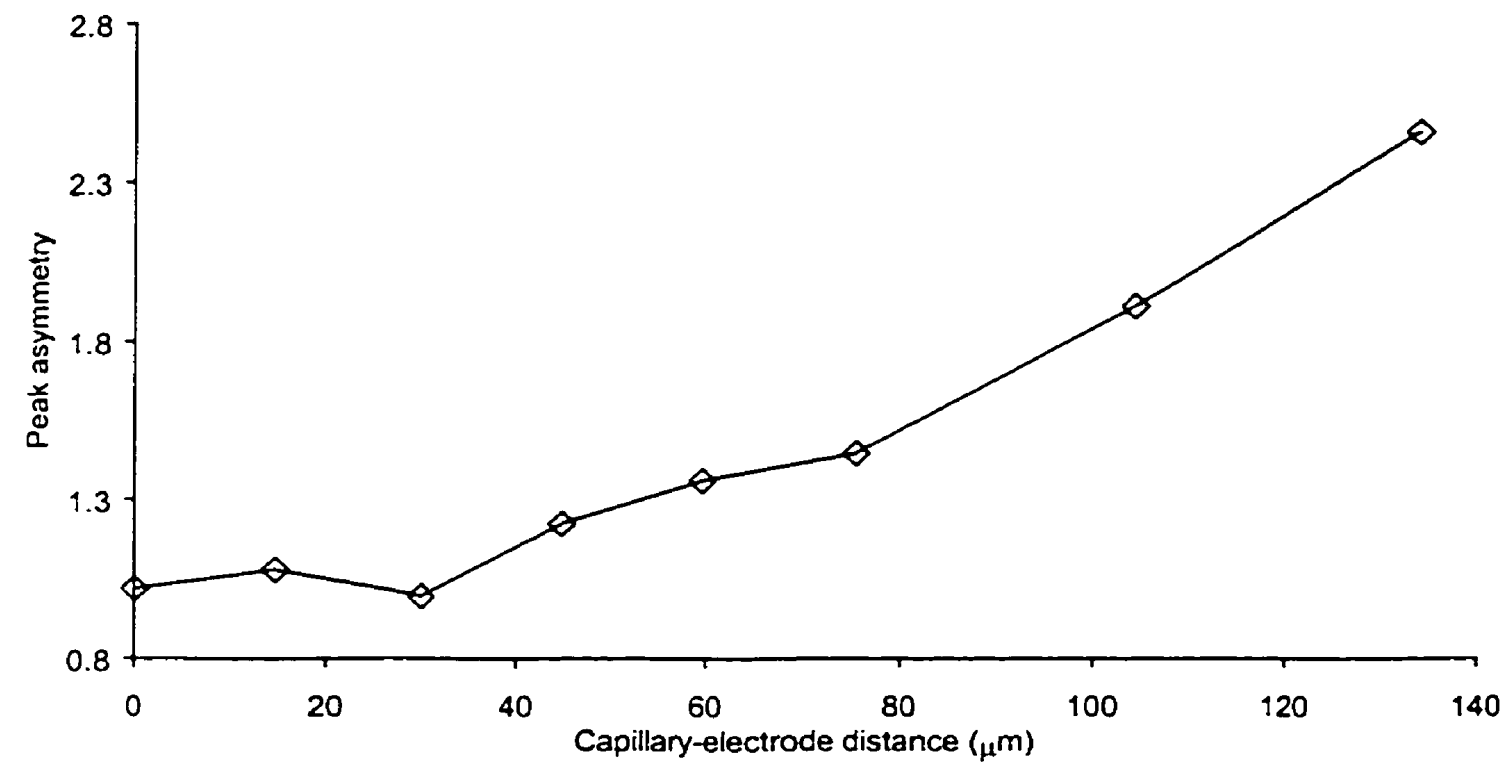

Figure 1.13 - Effect of capillary-electrode distance on peak asymmetry for the sulfonamide antibiotic trimethoprim. See Figure 1.11 for conditions.

The results showed that peak tailing decreased as the capillary was brought closer to the electrode and eliminated (an asymmetry of 1 indicates a perfectly symmetrical peak) at capillary-electrode distances of less than $30 \mu \mathrm{m}$. This indicates that much of the 
broadening that contributes to these efficiency losses at close capillary-electrode distances is general zone broadening. The source of this general zone broadening is not well understood, but it may be due to the method used to calculate peak efficiency. If signals are lower at small capillary-electrode distances, but the analyte zone widths remain constant (i.e. peak widths), the peak width at half-height will be wider, resulting in a decrease in the calculated number of theoretical plates $\left[\mathrm{N}=5.54\left(\mathrm{t}_{\mathrm{R}} / \mathrm{W}_{1 / 2}\right)^{2}\right]$. It is also possible that the EOF generated between the end of the capillary and glass which seals the electrode is turbulent due to unequal flow at each wall (the differences in the soft-glass used for the electrode and the fused-silica capillary may result in different $\mathrm{Si}^{-} \mathrm{O}^{-}$surface concentrations). These unequal flows may generate turbulences significant enough to disturb analyte zones at small capillary-electrode distances.

The effect that capillary-electrode axial misalignment (Figure 1.7a) had on the trimethoprim response was also examined. The electropherograms shown in Figure 1.14 were obtained by intentionally misaligning the capillary and electrode by the distance indicated on each plot while maintaining a capillary-electrode distance (Figure 1.7b) of 30 $\mu \mathrm{m}$. Unlike the peak shape distortions observed as the capillary-electrode distance was increased, general zone broadening and a decrease in signal strength was observed as the capillary and electrode became more misaligned. This was to be expected since, even when the capillary and electrode are misaligned, the "detection volume" remains unchanged as the electrode remains close to the end of the capillary. 


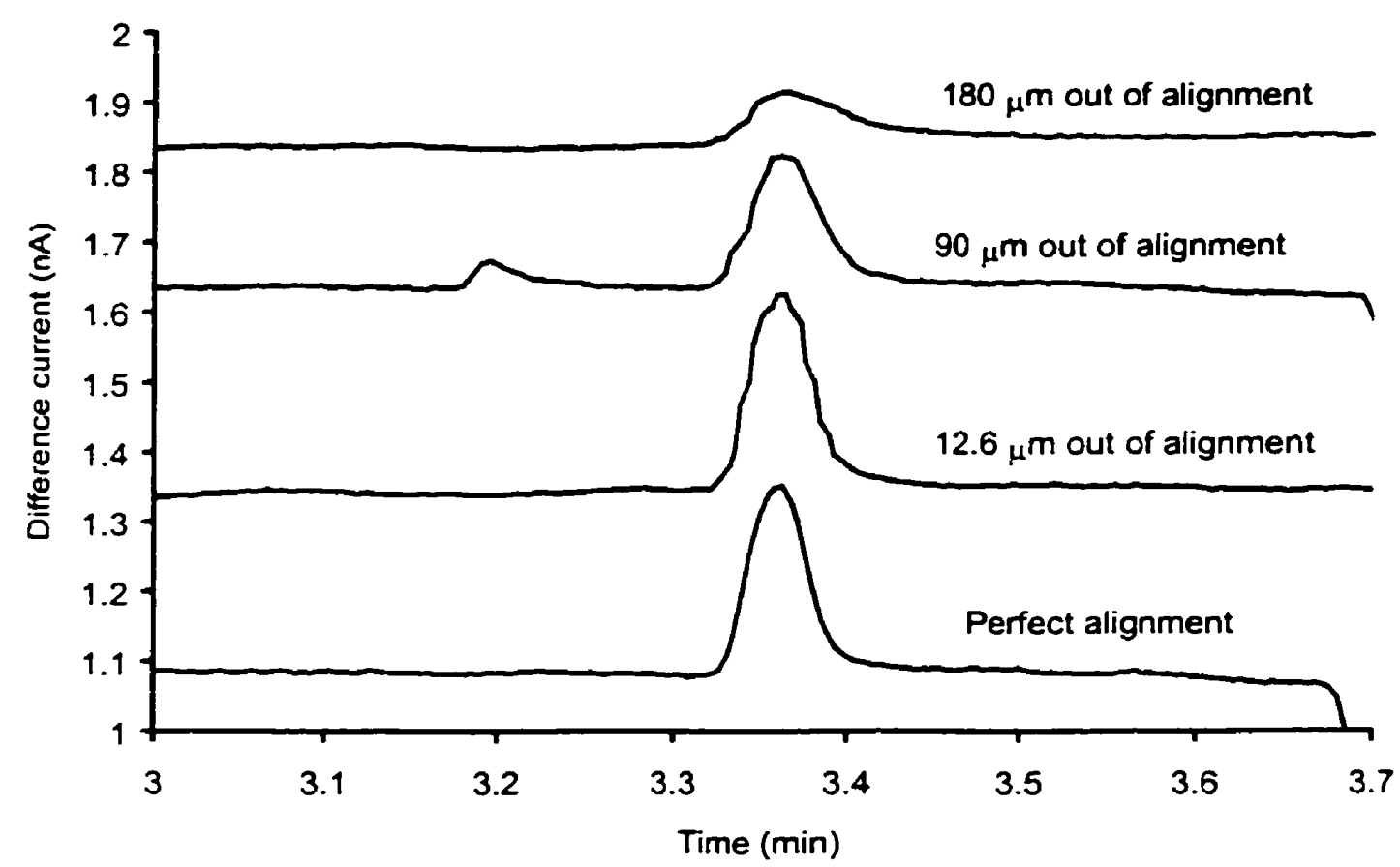

Figure 1.14 - Effect of capillary-electrode misalignment on trimethoprim electropherogram peak. Capillary-electrode misalignments indicated on each plot. See Figure 1.11 for conditions.

The magnitude of the peak distortion was not expected though. In the case where the capillary and electrode were misaligned by $12.5 \mu \mathrm{m}$ (the center of the electrode was aligned with the edge of the $25 \mu \mathrm{m}$ capillary opening) no peak shape distortion and only a negligible signal decrease were observed. This is contrary to what Lu et al ${ }^{25}$ observed when they studied the effect of capillary-electrode axial alignment on the amperometric observed for catechol separated by CE. They found that axial alignment had a much greater impact on signal strength. The important difference between these two studies is the detection method and electrode geometries that were used. In order to maximize the sensitivity of amperometric detection, Lu et al, and other researchers who have used this technique maximize coulometric efficiency by accurate placement of a carbon-fiber 
electrode. By inserting a cylindrical electrode directly into a capillary, coulometric efficiencies approaching $100 \%$ have been achieved ${ }^{16}$. Positioning the electrode outside of the capillary reduces the influence of the separation field, but also results in decreased efficiencies and lower sensitivities. This signal-dependence of capillary-electrode alignment observed by Lu et al for electrodes positioned outside the capillary illustrates the impact that coulometric efficiency losses have on the ultimate sensitivity of the detection method. In the case of SWV detection using a disk electrode, it appears that coulometric efficiency is not as important. Where the capillary and electrode were misaligned by $90 \mu \mathrm{m}$ (Figure 1.14), only a $20 \%$ loss in signal intensity was observed whereas Lu et al observed a $90 \%$ loss in signal intensity for a similar misalignment. A misalignment of $90 \mu \mathrm{m}$ exists when the electrode is positioned halfway between the center of the capillary and its outer wall. In this situation, the electrode will be exposed to a much smaller fraction of the analyte that exits the capillary (an accurate estimation of the decrease in analyte exposure to the electrode is difficult due to the complexity of the calculation to determine the effective diffusion zone of the electrode). It, therefore, appears that for SWV detection the electrode response is largely concentration dependent. Using amperometric detection, where coulometric efficiency appears to effect the ultimate $\mathrm{S} / \mathrm{N}$ to a larger extent, a more significant mass-dependence is observed.

The capillary-electrode alignment and distance study discussed above was performed using a separation electrolyte of $\mathrm{pH}$ 7.0. At this $\mathrm{pH}$, a reasonably fast EOF was observed. Although the generation of EOF diminishes at the end of the capillary, the bulk flow generated within the capillary will force fluid that has exited the capillary and is in 
the detection zone out of this zone. It is this action that permits the off-capillary detection schemes first reported by Wallingford et $\mathrm{al}^{13}$, where the EOF generation stops at the decoupling joint, but the capillary contents are pushed through the detection capillary by bulk flow generated within the separation field. In the absence of an EOF, migration of an analyte from the end of the capillary to the bulk solution would be dependant upon the $i \mathrm{R}$ drop that exists between the end of the capillary and the terminating electrode of the highvoltage power supply (the auxiliary electrode). It is this $i \mathrm{R}$ drop that will determine the magnitude of the field that will exist and hence the velocity of charged analytes within this field. As illustrated in Figure 1.10, this field will change with the positioning of the electrode and capillary. Because removal of an analyte from the "detection zone" will depend largely upon migration of the analyte within this low-field environment when a low-EOF exists, it was expected that capillary-electrode alignment and distance would be more of a factor in maintaining separation efficiency when low $\mathrm{pH}$ electrolytes were used which have only negligible EOF. A study of the effect of capillary-electrode positioning on signal-to-noise and peak shape was done using a low-pH electrolyte $(20 \mathrm{mM}, \mathrm{pH} 3$ phosphate buffer) and suitable probe, Deprenyl. The effect of capillary-electrode alignment and distance on the potential shift observed at the electrode when the separation voltage was applied are shown in Figure 1.15 and Figure 1.16. As these figures illustrate, the potential offsets observed with low $\mathrm{pH}$ separation electrolytes were similar to those observed for the $\mathrm{pH} 7$ separation electrolyte. Because peak shape distortions will be most evident for narrow peaks, electrophoretic conditions were manipulated to generate peak widths (at half-height) of 1-2 s. 


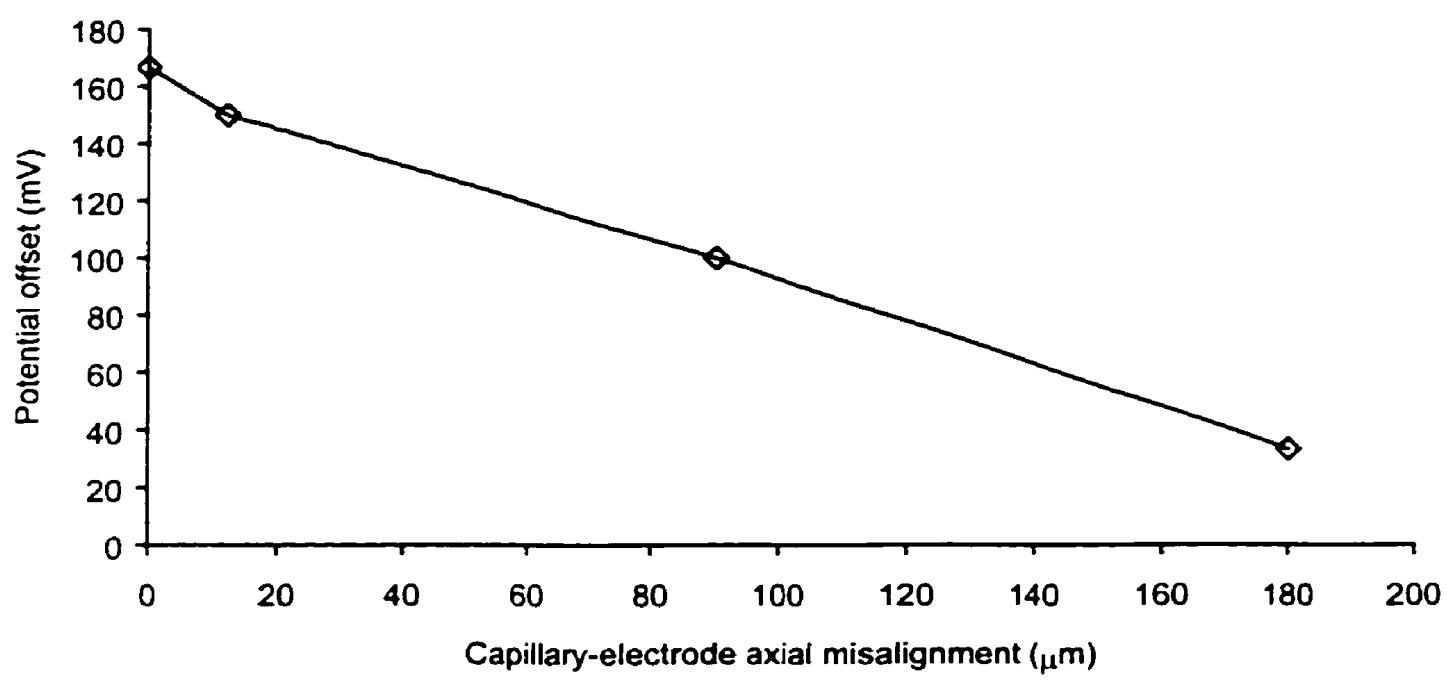

Figure 1.15 - Effect of capillary-electrode alignment on the potential shift observed at the electrode when a $10 \mathrm{kV}$ separation voltage was applied using a low-pH separation $(20 \mathrm{mM}, \mathrm{pH} 3.0$ phosphate buffer $)$ electrolyte.

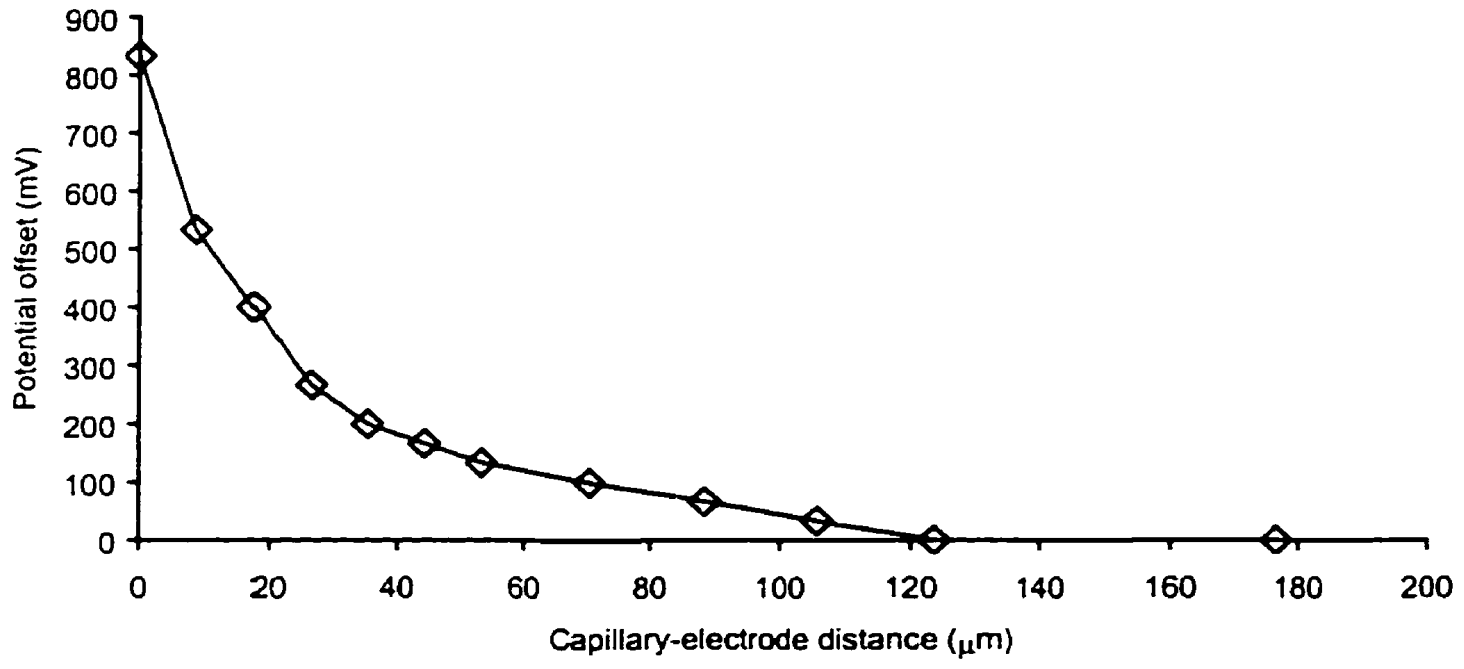

Figure 1.16 - Effect of capillary-electrode distance on the potential shift observed at the electrode when a $10 \mathrm{kV}$ separation voltage was applied using a low-pH $(20 \mathrm{mM}, \mathrm{pH} 3.0$ phosphate buffer $)$ separation electrolyte. 
Figure 1.17 shows, qualitatively, the effect of capillary-electrode distance on the peak shape of Deprenyl. The peak shape are distorted at both close capillary-electrode distances and beyond an optimal distance of $44 \mu \mathrm{m}$.

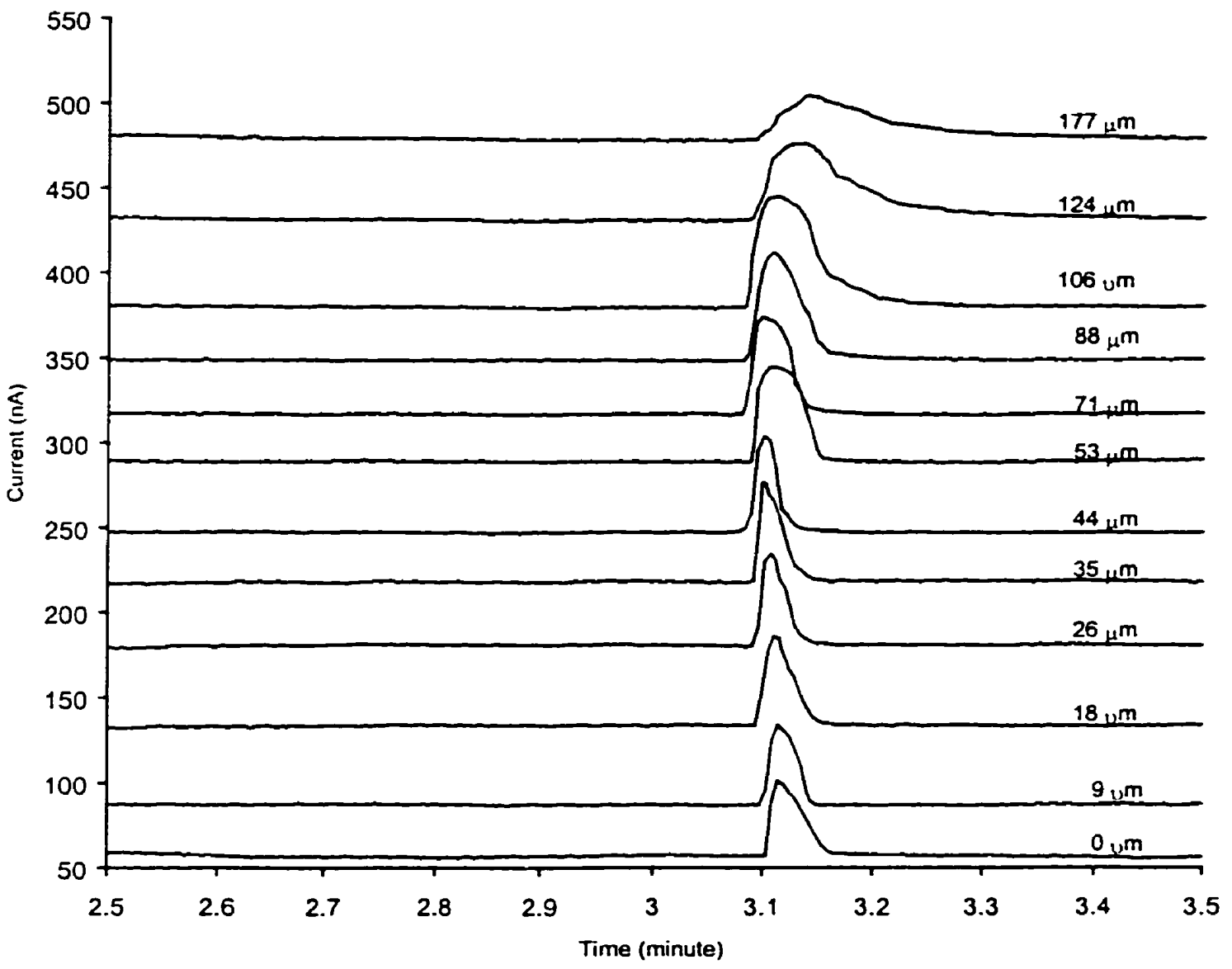

Figure 1.17- Effect of capillary-electrode distance on the peak shape of Deprenyl using $20 \mathrm{mM}$, pH 3.0 phosphate buffer as a separation electrolyte. Capillary-electrode distances are indicated on each plot. CE conditions: $2,0 \mathrm{mM}$ pH 3.0 phosphate buffer, $25 \mu \mathrm{m} \times 30 \mathrm{~cm}$ capillary, $10 \mathrm{kV}$ separation voltage, $1 \mu \mathrm{M}$ Deprenyl injected electrokinetically for $3 \mathrm{~s} @ 10 \mathrm{kV}$.

These capillary-electrode distance effects on peak width and $\mathrm{S} / \mathrm{N}$ are shown quantitatively in Figure 1.18. When the capillary was close to the electrode $(<40 \mu \mathrm{m})$, the 
decrease in $\mathrm{S} / \mathrm{N}$ is due to both a reduced response as well as increased noise. As the capillary is moved back from the electrode, the noise level decreases then remains constant at distances $>18 \mu \mathrm{m}$. The peak height increased steadily as the capillaryelectrode distance was increased, despite the peak becoming broader. It is unfortunate that the maximum $\mathrm{S} / \mathrm{N}$ occurs at a capillary-electrode distance $(106 \mu \mathrm{m})$ significantly larger than the capillary-electrode distance which produces an optimal peak shape $(27 \mu \mathrm{m})$.

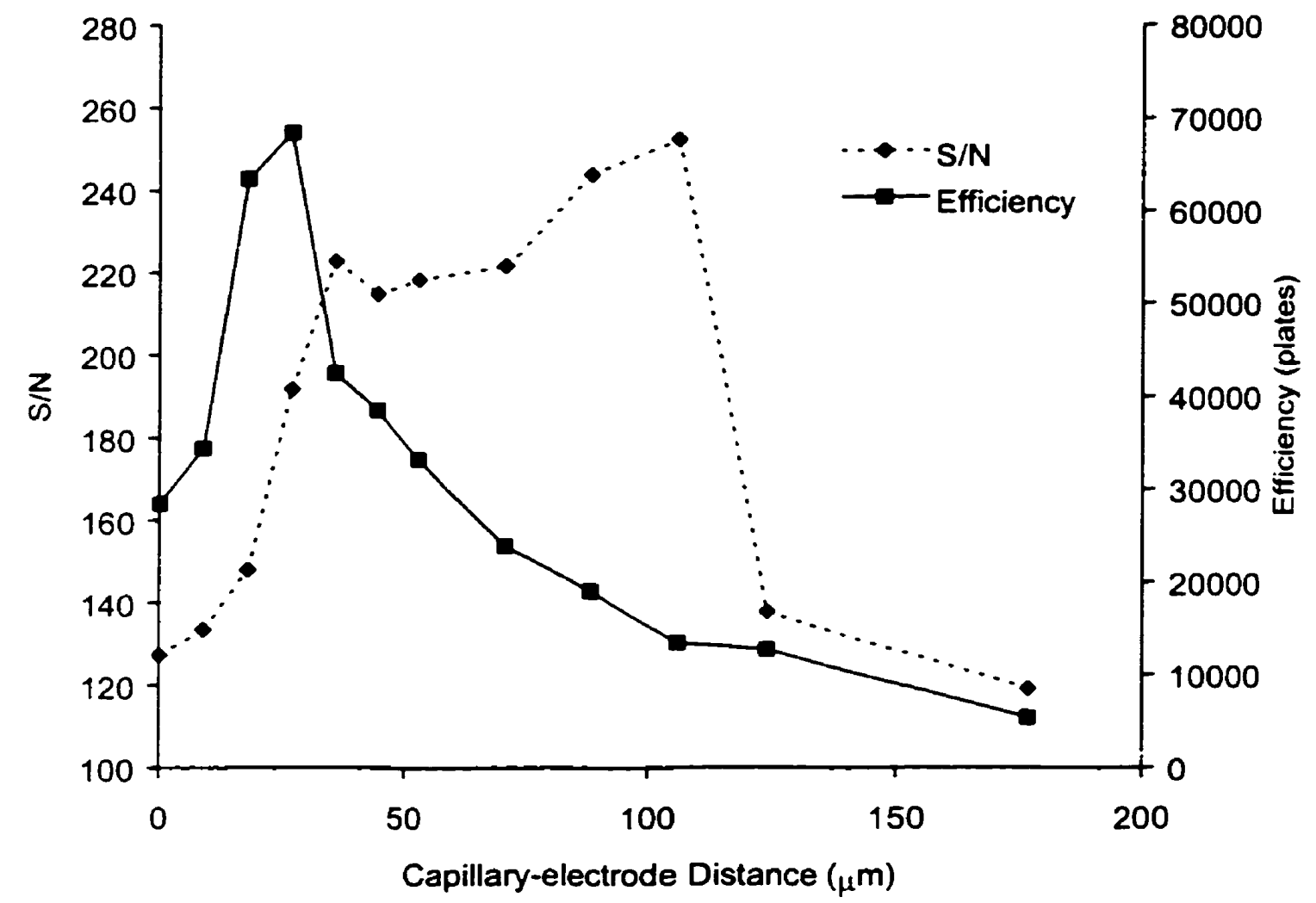

Figure 1.18 - Effect of capillary-electrode distance effects on Deprenyl peak efficiency and $\mathrm{S} / \mathrm{N}$ using a low-pH electrolyte. See Figure 1.17 for conditions.

As discussed above, these low-pH experiments were performed to investigate whether the negligible EOF produced using a low-pH separation electrolyte would make 
capillary-electrode alignment more critical. Although the effect of capillary-electrode distance on peak shape is almost identical for both low and high EOF electrolytes, it appears that when a low EOF electrolyte is used, response can be increased (at the expense of peak shape) by positioning the capillary farther from the electrode. When a high EOF exists, the analyte is swept out of the detection area regardless of the capillary-electrode distance. When little or no EOF exists, at larger capillary-electrode distances the analyte may linger in the detection area increasing its interaction with the electrode resulting in better coulometric efficiency. These experiments were done under electrophoretic conditions which produced relatively narrow peak widths. In more typical separations where analytes have migration times of the order of 5-10 minutes and peak widths $>5 \mathrm{~s}$, it may be possible to operate with capillary-electrode distances $>27 \mu \mathrm{m}$ without significant degradation of peak shapes while realizing the observed increased $\mathrm{S} / \mathrm{N}$ effects. Again, similar to experiments performed using a pH 7 separation electrolyte, the effect of capillary-electrode axial alignment was observed using the low-pH electrolyte. Electropherograms collected at various misalignments are shown in Figure 1.19. Unlike the peak distortions that were observed for capillary-electrode misalignment under high EOF conditions, peak asymmetries are observed under low-EOF conditions. As was discussed earlier, under low-EOF conditions, migration of the analyte out of the detection zone will occur more slowly as the field is reduced. As the electrode moves away from the center of the capillary, the field is reduced and peak tailing results (in addition to the general zone broadening observed under high-EOF conditions). 


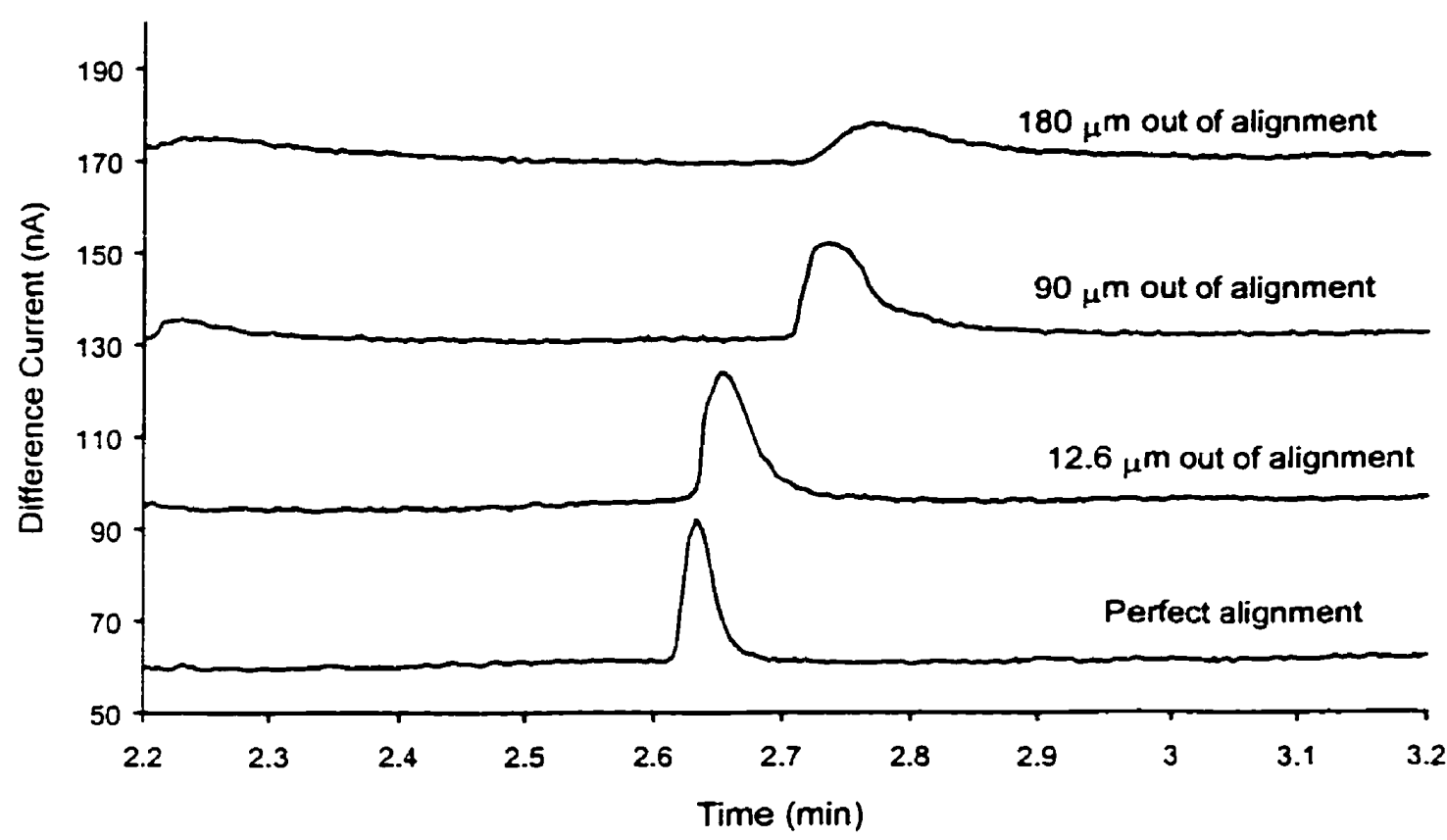

Figure 1.19 - Effect of capillary-electrode axial alignment on Deprenyl peak shape observed using the low-pH electrolyte. Electropherograms collected at various misalignments are shown. See Figure 1.17 for conditions.

\subsubsection{Effect of capillary internal diameter}

As indicated in the experimental section, separation capillaries with i.d. of $25 \mu \mathrm{m}$ were used for the capillary-electrode distance and alignment studies described above. When capillaries with larger $(50 \mu \mathrm{m})$ and smaller $(10 \mu \mathrm{m})$ i.d. were used, several changes were observed. As expected, the potential shift produced at the working electrode increased with increasing capillary diameter. After determining the optimal capillaryelectrode distance for 10 and $50 \mu \mathrm{m}$ capillaries (i.e., distance at which $\mathrm{S} / \mathrm{N}$ and peakefficiency were optimal), sulfonamide separations using these capillaries were compared to those obtained using a $25 \mu \mathrm{m}$ capillary (Figure 1.20). 


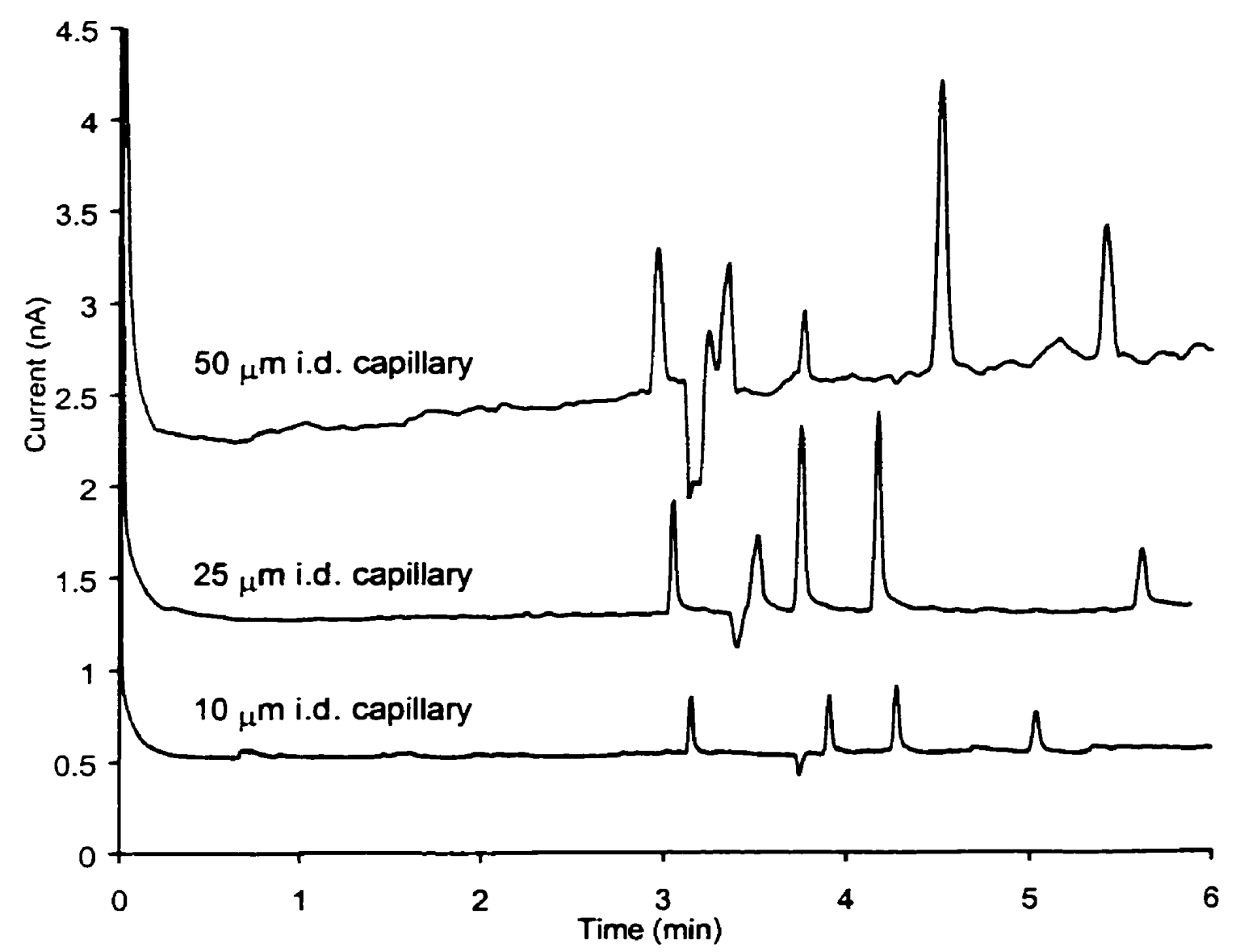

Figure 1.20 - Effect of capillary diameter on the separation of sulfonamide antibiotics. See Figure 1.11 for conditions.

Although $\mathrm{S} / \mathrm{N}$ and general baseline stability observed for $10 \mu \mathrm{m}$ i.d. capillaries were similar to those that had been obtained using $25 \mu \mathrm{m}$ i.d. capillaries, $10 \mu \mathrm{m}$ i.d. capillaries were troublesome to use as they required significant inlet vial pressure to rinse the capillary in a reasonable time and were more prone to plugging. The 5 p.s.i. inlet vial pressure provided by the automated CE described in Section 1.2.6 was not high enough to rinse the $10 \mu \mathrm{m}$ i.d. capillaries in a reasonable time, so a $3 \mathrm{~mL}$ plastic syringe with a capillary interface was used for rinsing $10 \mu \mathrm{m}$ capillaries. The electropherogram obtained 
using a $50 \mu \mathrm{m}$ i.d. capiliary (Figure 1.20) was one of the better quality electropherograms that were collected during a series of injections. Signal-to-noise was lower and baseline drifting was much more pronounced when $50 \mu \mathrm{m}$ i.d. capillaries were used. This noise and drifting is likely due to an increased electrophoretic current which produced more electrolyte decomposition products within the small-volume CE-ECD cell. These impurities can migrate into the separation capillary and past the working electrode thus causing baseline drifting and other low-frequency noise. Based on these observations, 25 $\mu \mathrm{m}$ i.d. capillaries appear to be both the easiest to use and provide the best $\mathrm{S} / \mathrm{N}$ and baseline stability. It is interesting to note, though, that the analyte signal increases as the capillary i.d. increases. This would indicate that the detection process is not simply concentration-dependent, but has some mass-dependence as well.

\subsubsection{An improved electrode positioning system}

Initially, when a CE-ECD cell was assembled, the capillary and electrode were aligned using visual inspection while adjusting the alignment screws. The capillaryelectrode distance and alignment studies discussed above showed that the position of the capillary and electrode could be determined from the potential shift of a SWV voltammogram as adjustments were made. Axial alignment was achieved by adjusting the alignment screws along both axes until the maximum potential shift was observed. Similarly, rather than trying to set the capillary-electrode distance to an absolute spatial value known to be optimal, the optimal capillary-electrode distance was recorded as a potential shift rather than a spatial distance. When the capillary-electrode distance needed to be reset, it was adjusted until this optimal potential shift was observed. These potential 
shift-based electrode-capillary positioning techniques were found to be more precise and less time consuming than previously used optical methods.

\subsubsection{Repeatability studies}

The repeatability of the CE-ECD automated instrument, baseline precision and general stability of the ECD system over time were investigated using a series of replicate injections. These results are shown in Table 1.1.

Table 1.1 - Results of CE-ECD reproducibility investigations.

\section{Peak height RSD}

Injection-to-injection ${ }^{2}$

After capillary-electrode repositioning ${ }^{b}$

Day-to-day ${ }^{\mathrm{c}}$
$3.6 \%$

$3.3 \%$

$13 \%$
Peak area RSD

$6.7 \%$

$6.3 \%$

$8.0 \%$

${ }^{i} 10$ replicate injections.

${ }^{b} s$ injections where before each injection, the electrode was removed from the cell, then reinserted and realigned.

' 5 replicate injections were made on 5 consecutive days.

Having developed a procedure for accurately positioning the capillary and electrode, repeatability studies were performed to determine the precision that could be obtained for analyses performed after capillary-electrode re-alignment. The capillaryelectrode alignment and distance was randomly changed and then reset before each injection using the potential-shift procedure described above. Results of this study are shown in Table 1.1. The similar precisions obtained for injection-to-injection repeatability and repeatability after electrode realignment shows the alignment repeatability is $<3 \%$. 
The day-to-day variability of the system was evaluated from replicate injections on successive days after the CE-ECD system had stabilized. Before the system was used each day, the capillary was rinsed with $0.1 \mathrm{M} \mathrm{KOH}$ for $2 \mathrm{~min}$ followed by the separation buffer for $2 \mathrm{~min}$. After the system appeared stable (typically, 2-3 injections were required to stabilize the CE-ECD system after startup each day), replicate injections were performed. As the data in Table 1.1 show, excellent day-to-day precision was obtained. Since this precision was obtained without any electrode conditioning (i.e. mechanical polishing, voltage cycling), these results also show that the electrode is stable for this mode of detection.

\subsection{Conclusions}

The CE-ECD instrumentation reported here allows relatively easy and reproducible alignment of a $\mu \mathrm{m}$-scale disk electrode with the end of the separation capillary and, when used with square-wave voltammetric detection, allows sensitive electrochemical detection. Once the capillary was fastened into the CE-ECD cell and aligned with the electrode, manipulation of the capillary and typical laboratory bench vibrations did not perturb this alignment. The working/auxiliary electrode system that was developed was rugged and did not require enclosure in a faraday cage. Although the CEECD cell described was compact enough to be accommodated into most commercial automated $\mathrm{CE}$ instruments, the electronic configuration of most commercial CE instruments precludes ECD. A reproducibility study of an in-house constructed automated CE-ECD instrument showed that the general reliability of the CE-ECD system was good even after re-alignment of the capillary-electrode was performed. These results show that 
the design of this CE-ECD system offered significant advantages over ECD with fragile cylindrical carbon-fiber electrodes used in off-capillary detection format. 


\section{CHAPTER 2 - SQUARE-WAVE VOLTAMMETRY DETECTION FOR CE}

\subsection{Introduction}

As indicated in the previous chapter, amperometric detection has been the most popular ECD mode used for $\mathrm{CE}$. In amperometric detection, an electrode is maintained at a constant potential at which analytes of interest are known to react. The current generated by electrode reactions is converted to a voltage, amplified and plotted. The popularity of amperometric detection is due to a number of reasons:

i) The electronic instrumentation required for amperometric detection (potentiostat, current amplifier, reference electrode) is simple and available commercially.

ii) Fabrication of a $\mu \mathrm{m}$-scale working electrode suitable for interfacing with a CE capillary is relatively easy.

iii) The output of the amplified signal requires no further processing and can simply be plotted to obtain an electropherogram.

iv) The implementation of amperometric detection in flowing systems is well understood and has been used in HPLC for over 25 years to provide excellent detection sensitivity for electrochemically active analytes ${ }^{12}$.

For these reasons, when Wallingford and Ewing first applied ECD to $C E^{13}$, amperometric detection was an obvious choice of ECD mode to use. Since then, amperometric has also been the mode of choice in most subsequent CE-ECD applications $^{31,32.33}$. However, other ECD methods have been reported for CE. Potentiometric detection was one of the first detection methods reported for zone electrophoresis. In 1974, Virtanen ${ }^{34}$ used changes in the potential of a Ag/AgCl-coated $\mathrm{Pt}$ 
wire to detect small inorganic ions separated in a $200 \mu \mathrm{m}$ capillary. More recently, Harber et al used potentiometry to detect alkali and alkaline-earth metals separated at concentrations below $100 \mathrm{nM}^{35}$. This same research group also reported potentiometric detection of various inorganic ions with a coated electrode ${ }^{36}$. Although potentiometric detection has provided good sensitivities for analytes that do not respond to more traditional CE detection methods, it is limited to a narrow range of analytes. Also, peak shape distortion has been a problem. Conductivity detection has also been successfully applied to $\mathrm{CE}^{37.38}$, and a commercial CE-conductivity detector is available. Like potentiometric detection, conductivity detection has provided good sensitivity for small, high-mobility ions, but has rather limited applicability. Because these non-faradic ECD methods (potentiometry and conductivity) measure bulk solution properties, drifting baselines can become a problem when used in flowing systems.

Voltammetric detection has been reported for both HPLC $39,40.41$ and $\mathrm{CE}^{42.43}$, but has generally been considered less sensitive than amperometric detection. Unlike amperometric detection where the working electrode current is measured at a constant potential, voltammetry ramps the potential of the electrode across a defined potential region and samples the current across this region to obtain a potential-dependant current response. When voltammetry is used as a detection method in a chromatographic system, the current response from each potential scan must be interpreted/integrated to generate one chromatographic data point. The authors of these reports concede in their introductions that amperometric detection is inherently more sensitive but offer that the additional qualitative data that voltammetric detection provides may offset its lack of 
sensitivity. This perception that amperometric detection is inherently more sensitive than voltammetric detection may not be accurate. Although reports describing voltammetric detection in flowing systems usually report lower sensitivities, this may be due to the instrumentation used rather than inherently poorer sensitivity. The typical rationale for the poorer sensitivity is that the charging current encountered in voltammetric detection interferes with the measurement of faradic current. Charging currents are generated each time the potential of the working electrode is changed because the ionic double layer changes its composition to accommodate the change in charge density on the electrode surface. The charging current is initially large, and then quickly decays to zero. The size of the electrode and the conductivity of the electrolyte will, to a large part, determine the rate of this decay.

The measurement of faradic currents in the presence of large charging currents has always been a challenge. For simple voltammetric techniques, such as linear-sweep voltammetry where the potential is simply ramped from an initial potential to a final potential, the charging current associated with each potential step is a major contribution to the overall current measured. A number of modifications to the simple linear-sweep voltammetric technique have been introduced in an attempt to minimize this charging current interference (Figure 2.1). 

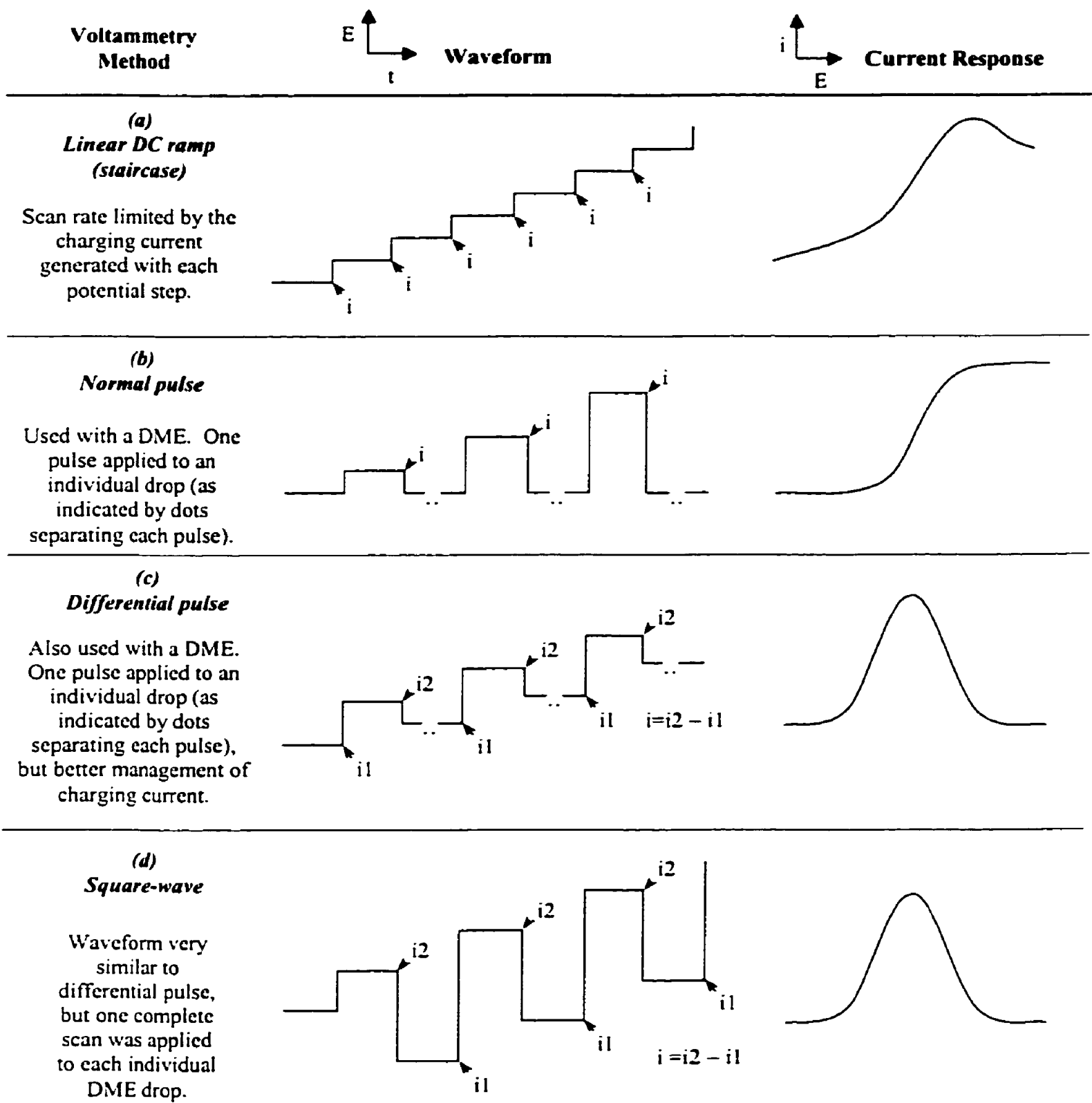

Figure 2.1 - Pulsed voltammetric techniques.

Typically, these modifications use potential pulses superimposed upon the DC potential scan. The fact that charging current will decay exponentially $\left(i_{\text {charging }} \alpha e^{-t / R C}\right)$ while faradic current decays $\alpha \mathrm{t}^{-1 / 2}$ (assuming linear diffusion) ${ }^{4+}$, is exploited by measuring current late in the potential pulse when the charging current has dissipated. The features 
of these voltammetric techniques are illustrated in Figure 2.1. Modern square-wave voltammetry (SWV, Figure 2.1d) is the culmination of improvements made to these pulsed linear-sweep techniques. In SWV the current measured at the end of the reverse pulse is subtracted from the current measured at the end of the forward pulse, and thus the contribution of the charging current to the overall current is significantly reduced. To minimize the charging current response in SWV, high ionic strength electrolytes and small electrodes are often used. High ionic strength electrolytes redistribute the composition in the ionic double layer faster and although higher initial currents will be observed, charging currents will decay more quickly.

Before the introduction of $\mu \mathrm{m}$-scale electrodes, the use of highly conductive electrolytes was the primary method used to reduce the effect of charging currents, but decreasing the size of the working electrode is also useful. For mm-scale and larger electrode systems, the electrode surface can be approximated as planar regardless of electrode shape. Such electrode dimensions are significantly larger than the diffusion distance of molecules for typical time scales that are used for these techniques (i.e. $\sim 1$ s). When the electrode size is reduced to $\mu \mathrm{m}$-scale dimensions, even for time scales less than I s, the dimensions of the electrode are much smaller than the diffusion distance for molecules in a solution. This has significant implications on the transport of molecules to the electrode surface. The faradic current response in a diffusion-controlled process will depend upon how fast the analyte can be supplied to the electrode. If the potential of a planar electrode is stepped from a potential where an analyte in solution does not react, to a potential where it does, analyte consumed at the electrode surface by the electrochemical 
reaction will create a concentration gradient that will progressively extend out into the solution as electroactive analyte is consumed. The faradic current response for an electrode of these dimensions will initially be high, but will then decay to a low, steadystate current. The decay rate and magnitude of this steady-state current will depend upon the transport mechanisms (i.e., if convection exists) occurring in the system. When $\mu \mathrm{m}$ scale electrodes are used the diffusion zone will have a hemispherical shape (i.e., for hemispherical or disk-shaped $\mu \mathrm{m}$-scale electrodes). As a result, when the diffusion zone expands out from the electrode during electrolysis, the surface area of molecules "feeding" the electrode also expands. At the point where the flux of molecules through this hemispherical area can supply the electrode surface with reaction material, the diffusion zone will stop its expansion and a high, steady-state current will be maintained. It is this rapidly reached steady-state current that makes $\mu \mathrm{m}$-scale electrodes attractive for use in SWV. In response to an applied potential the charging current will decay exponentially to zero while the faradic current will reach a steady state. Using traditional SWV, the frequency of the square-wave can be adjusted so that measurement of the faradic current (at the end of each forward and reverse pulse) is made after the charging current has decayed and the faradic current has reached steady state. SWV experiments performed in this manner should produce current measurements equal in magnitude to amperometric current measurements the latter are also made when faradic currents have reached steady state conditions and charging currents were zero. However, due to the different decay rates of charging and faradic currents, it is possible to adjust the square-wave frequencies to allow higher faradic currents to be measured in the presence of moderate charging 
currents. This enables one to maximize the ultimate $\mathrm{S} / \mathrm{N}$ of the technique, resulting in higher sensitivities than are obtainable with amperometric detection. In addition, SWV can also provide qualitative data regarding the potential dependence of the current response which may allow better discrimination between the analyte and interfering background responses. This allows for an increase in $\mathrm{S} / \mathrm{N}$ that is not possible with amperometric detection.

The ability to maximize the sensitivity of SWV also depends upon the use of instrumentation that will enable complete control of both current measurement timing and ivaveform parameters. Early application of pulsed voltammetric techniques was limited by the electronic instrumentation available which relied on analog electronics to generate waveforms iwhile synchronously collecting current data ${ }^{45}$. Later applications benefited from the precision, speed and flexibility of computer-controlled digital waveform generation and current sampling equipment which allowed easy manipulation of the many operational parameters. These advances in data acquisition/control hardware have allowed application of SWV detection in modern flowing systems (i.e. chromatographic and flow-injection systems).

SWV was successfully applied to flow injection for the analysis of iodide in food and environmental matrices ${ }^{46}$. The detection limits reported were comparable to other detection methods, and better discrimination of iodide within the complex matrix was achieved. SWV has also been used for HPLC ${ }^{47}$ detection. Samuelsson et al reported the first application of SWV to HPLC in $1980^{48}$. Although the authors of this report admitted 
that detection limits obtained with SWV were higher than those reported using other ECD techniques, the possibility of using SWV to discriminate between analytes with different reaction potentials based upon their unique voltammetric response was illustrated. Goto and Shimada used SWV detection to detect catecholamines separated by HPLC ${ }^{39}$. They illustrated the utility of SWV to separately detect catecholamines not resolved by HPLC but which had unique voltammograms. When amperometric detection was used, only one peak was observed for a separation zone containing two analytes, whereas from the 3dimensional time-potential-current representations of the separations performed using SWV detection it could be determined that two analytes with different oxidation potentials were co-eluting. Because this application focused more on the qualitative benefits of HPLC-SWV, detection limits were not discussed. Roush and Anderson ${ }^{47}$ reported the use of SWV for gradient elution HPLC. They noted that mobile phase changes during gradient elution caused baseline drifting with amperometric detection due to the changing conductivity of the electrolyte. Although no direct comparisons were made between amperometric and SWV detection, chromatograms using SWV detection for gradient elution HPLC did not have drifting baselines. The authors also noted the utility of HPLCSWV for determining the peak-purity. Detection limits reported were an order of magnitude higher than previous ECD methods used for the detection of similar compounds.

In addition to difficulties in obtaining adequate sensitivities, these reports indicated that maintaining temporal resolution of the separation technique was also a challenge with SWV detection. The time required to complete the potential scan will dictate the ultimate 
sample rate of SWV detection. For modern HPLC, where peak widths of $<10$ s are typically encountered, sampling rates in the order of $>0.5 \mathrm{~Hz}$ are required to obtain reasonable peak information (20 data points) for quantitation. All the SWV detection methods discussed above used scan times significantly higher than the scan time required for reasonable quantitation in routine HPLC. There were several reasons why longer than optimal scan times were used. The basic SWV waveform consists of a potential ramp, which, when applied using a digital interface will consist of a series of potential steps. At each potential step, a square-wave will be applied. The ultimate scan time will be determined by the width of the potential window and square-wave frequency. While the potential window can be made as narrow as possible and still contain the voltammogram curves for the analytes of interest, the square-wave frequency that can be used will depend upon several experimental and instrumental factors. As discussed above, the conductivity of the electrolyte and size of the electrode will have a major impact on the faradic/charging current ratio. When mm-scale electrodes are used, lower square-wave frequencies must be used or charging currents will not decay before faradic currents are measured. When lower square-wave frequencies are used, longer scan times must also be used as each potential step becomes longer to accommodate each square-wave period. Although a square-wave amplitude of $50 \mathrm{mV} / \mathrm{n}$ has been shown to be optimal theoretically ${ }^{45}$, lower amplitudes can be used to reduce charging currents (at the expense of sensitivity). The HPLC-SWV applications reported by Samuelsson et al ${ }^{+8}$ and Roush and Andersen ${ }^{47}$ used $\mathrm{mm}$-scale sensing electrodes, and as a result were limited to using square-wave frequencies of $10-100 \mathrm{~Hz}$, which resulted in scan times of 2-5 s 
(chromatographic data acquisition rates of $0.5-0.2 \mathrm{~Hz}$ ). Goto et $\mathrm{al}^{39}$ used $\mu \mathrm{m}$-scale carbon fiber electrodes, in their ECD cell (a $3 \mathrm{~mm}$ carbon fiber electrode was inserted into a narrow-bore capillary). While suitable for amperometric detection, this cell design likely resulted in high $i \mathrm{R}$ drops for $\mathrm{SWV}$ detection and limited the square-wave frequencies that could be used (square-wave frequencies of $15 \mathrm{~Hz}$ and scan times of $15 \mathrm{~s}$ were reported). As a result, although SWV gave more qualitative data, the quantitative use of these applications was limited. Kounaves and Young reported a HPLC-SWV application with $\mu \mathrm{m}$-scale carbon fiber electrodes with a square-wave frequency of $600 \mathrm{~Hz}$ to obtain a chromatographic data acquisition rate of $0.5 \mathrm{~Hz}^{49}$ (an ECD cell designed to reduce $i \mathrm{R}$ drop permitted these higher square-wave frequencies). While the detection limits were an order of magnitude higher than had been reported using amperometric detection, data acquisition rates of this order permitted quantitative analysis for HPLC. Although this application of SWV provided the most sensitivity and highest temporal resolution of all voltammetric detection modes applied to flowing systems, for application to CE where peak widths of $2 \mathrm{~s}$ are not uncommon, chromatographic data acquisition rates of at least an order of magnitude higher are required. It should be noted here that, although additional qualitative peak data can be obtained using voltammetric detection, it is of only limited applicability. In contrast to the additional resolving power that can be obtained using scanning UV absorption detection in chromatography, due to the varied wavelength dependence of absorbing analytes, very few applications of analytical importance exist where significant enough voltage dependence of the analytes' current response would allow separate identification/quantification of co-eluting species. For this reason, 
judgment of successful application of SWV as a CE detection method must be primarily based upon detection sensitivity and the range of analytes that can be detected. Thus, to warrant the added complexity of the SWV system, its application must provide a detection system that is clearly superior to the simpler amperometric system.

The challenge in this work was to develop a CE-SWV detection system that could provide both the temporal resolution and sensitivity required by $\mathrm{CE}$. Because no commercial instrumentation existed for SWV application to CE, a data acquisition and control system was developed in-house. A flexible SWV data acquisition/control system was integral to the application of SWV to CE and its development is described in Appendix A. The instrumentation described in Chapter 1 provided a CE-ECD system that could accoinmodate the square-wave frequencies that were necessary to achieve the temporal resolution demanded by $\mathrm{CE}$. Using this $\mathrm{CE}-\mathrm{ECD}$ system, a comprehensive examination of CE-SWV was performed in order to maximize both temporal resolution and sensitivity. Using chromatographic $\mathrm{S} / \mathrm{N}$ as an indicator, a systematic examination of the various SWV parameters was performed using the neurotransmitters dopamine and epinephrine as test compounds. Several CE-ECD reports using both amperometric and voltammetric ECD modes have been reported for these neurotransmitters, to which comparisons were made. One limitation of amperometric detection has been the gradual sensitivity losses that accompany electrode fouling. Post-scan pulses were incorporated into the SWV waveform to investigate whether continuous conditioning of the electrode surface could be used to maintain a clean electrode. Also, pre-scan pulses were added to investigate the utility of pre-concentrating analyte on the electrode surface prior to the 
SWV scan to enhance sensitivity. Experiments where performed to optimize the potentials and times of these pre- and post-scan pulses.

\subsection{Experimental}

\subsubsection{Chemicals}

All solutions were prepared from water purified using an ULTROpure with a TFM membrane and NANOpure II with Type I ORGANIC free cartridge kit (Bamstead Corp., Dubuque, IA). Unless otherwise stated, all reagents were analytical grade. Dopamine and epinephrine were obtained from Aldrich Chemical Co. (Milwaukee, WI).

\subsubsection{Automated CE-ECD system}

Sections 1.2.2-1.2.6 describe the automated CE-ECD system used. Appendix A describes in more detail the data acquisition system used.

\subsubsection{CE Conditions}

Fused-silica capillaries ( $30 \mathrm{~cm}$ x $25 \mu \mathrm{m}$ i.d., $365 \mu \mathrm{m}$ o.d., Polymicro Technology, Phoenix, AZ) were used in these experiments. Before use each day, capillaries were rinsed with $0.1 \mathrm{M}$ potassium hydroxide for $5 \mathrm{~min}$ followed by the separation electrolyte for $5 \mathrm{~min}$. Also, before each injection, the capillary and CE-ECD cell were rinsed with -2-5 void volumes of the separation electrolyte. Sample injections were made electrokinetically by inserting the anodic end of the capillary in the sample solution and applying $5 \mathrm{kV}$ for $10 \mathrm{~s}$. Samples were diluted to the desired concentration using the CE separation buffer. 
The neurotransmitters dopamine and epinephrine were used to investigate CE-

SWV. Separation and SWV conditions are shown in Table 2.1

Table 2.1 - Separation and SWV conditions used for CE-SWV of dopamine and epinephrine.

\section{Separation conditions}

Electrolyte : $50 \mathrm{mM} \mathrm{Na} \mathrm{HPO}_{4}-\mathrm{Na}_{2} \mathrm{~B}_{4} \mathrm{O}_{7}$ at $\mathrm{pH} 7.0$

Separation potential : $30 \mathrm{kV}$

Capillary : $25 \mu \mathrm{m} \times 30 \mathrm{~cm}$ fused silica

SWV conditions

Pre-scan pulse : $-500 \mathrm{mV}$ for $30 \mathrm{~ms}$

Post-scan voltage : $1000 \mathrm{mV}$ for $30 \mathrm{~ms}$

DC ramp : $100-500 \mathrm{mV}$ in $56 \mathrm{~ms}$

Square wave : $2000 \mathrm{~Hz}, 50 \mathrm{mV}$

Quantitation method: Average peak current, first 30\% of forward and reverse pulse current response rejected, $70 \mathrm{mV}$ detection band width, $15 \mathrm{SWV}$ scans included in running average

\subsubsection{Calculations of $S / N$ and peak efficiency}

See section 1.2.8.

\subsection{Results and discussion}

\subsubsection{Electrode type and positioning}

To accommodate the $\mathbf{l} \mathbf{k H z}$ or higher square-wave frequencies necessary for $\mathrm{CE}$ -

$\mathrm{SWV}, \mu \mathrm{m}$-electrodes were used. Although cylindrical carbon-fiber electrodes inserted into the end of the separation capillary have been popular for the analysis of neurotransmitters, as noted in Chapter $\mathrm{I}$, the use of cylindrical $\mu \mathrm{m}$-electrodes in $\mathrm{CE}$ requires rather complex apparatus to decouple the high separation voltage from the detection system. Even using the decoupling apparatus, a potential gradient along the length of the carbon fiber may exist ${ }^{25}$. Although this may cause insignificant problems for 
fixed potential amperometric detection, it would cause broadening of the voltammogram peak in SWV. Also, design of a CE-ECD cell based upon a cylindrical carbon fiber electrode with a time constant low enough to prevent a disruptive $i \mathbb{R}$ drop at high squarewave frequencies would require serious compromises. Although it was difficult to construct carbon-fiber disk electrodes, 10 and $25 \mu \mathrm{m} \mathrm{Pt}$ and Au disk electrodes were constructed relatively easily and reproducibly. Pt electrodes have been used for the detection of neurotransmitters ", but no SWV reaction was observed with a $25 \mu \mathrm{m} \mathrm{Pt}$ electrode.

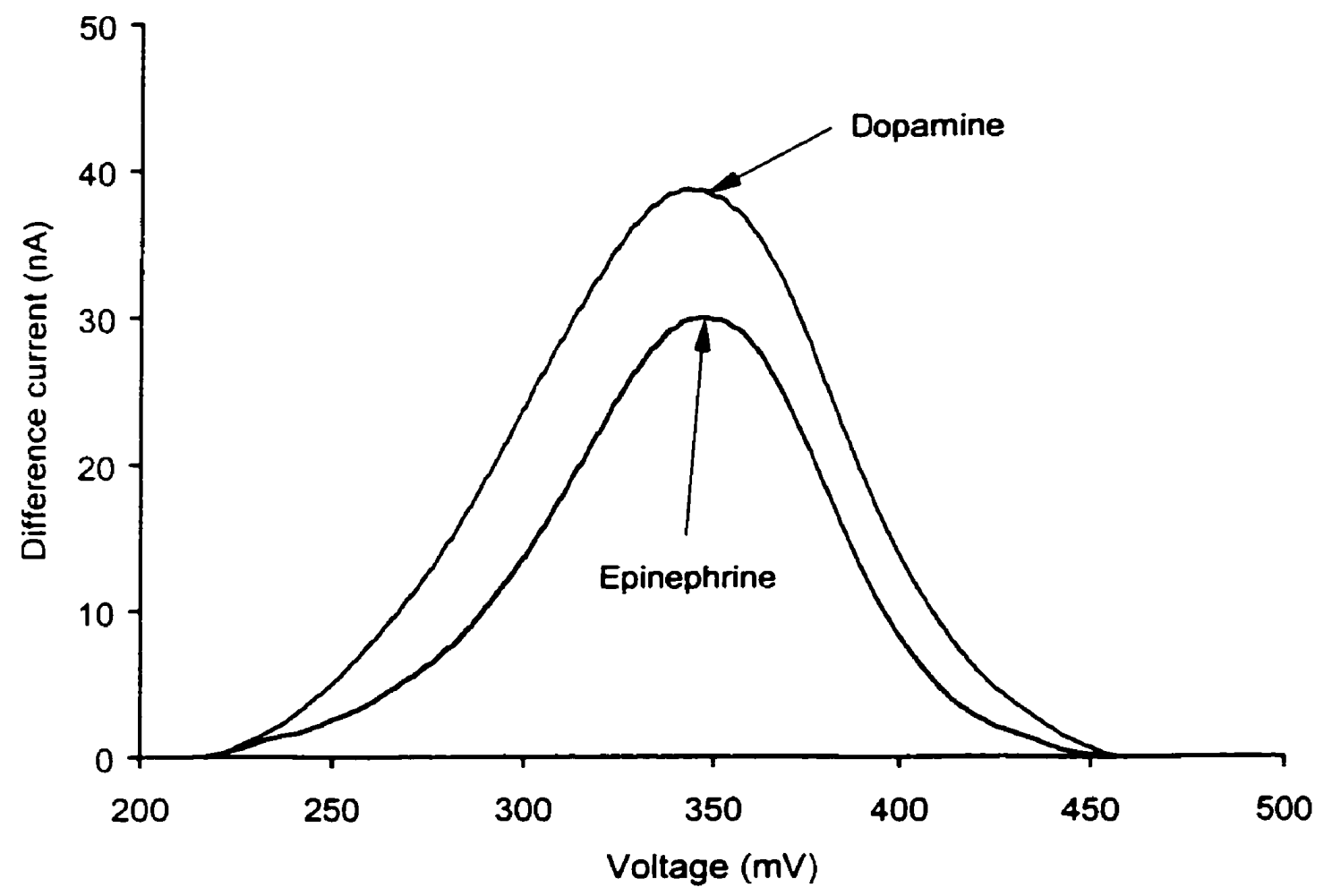

Figure 2.2 - Background subtracted square wave voltammograms of dopamine and epinephrine obtained by capturing the square wave voltammogram as the analyte peak migrated past the electrode. $\mathrm{CE}$ conditions: $50 \mathrm{mM} \mathrm{Na} \mathrm{HPO}_{4}-\mathrm{Na}_{2} \mathrm{~B}_{4} \mathrm{O}_{7}$ at $\mathrm{pH} 7.0,10 \mu \mathrm{M}$ dopamine and epinephrine injected $5 \mathrm{~s} @ 10 \mathrm{kV}$. SWV conditions: 200 to $500 \mathrm{mV}$ DC ramp in $56 \mathrm{~ms}, 2000 \mathrm{~Hz}, 50 \mathrm{mV}$ square-wave. 
A well defined SWV peak was observed for both dopamine and epinephrine using 10 and $25 \mu \mathrm{m}$ Au electrodes. Dopamine and epinephrine gave almost indistinguishable square-wave voltammograms using an Au electrode. Figure 2.2 shows background subtracted square wave voltammograms for these compounds obtained by capturing the square wave voltammogram as the analyte peak migrated past the electrode.

Although increasing $\mathrm{S} / \mathrm{N}$ should accompany decreasing electrode surface area, we obtained better $\mathrm{S} / \mathrm{N}$ using the $25 \mu \mathrm{m}$ Au electrode. This may have been due to smaller currents produced by the $10 \mu \mathrm{m}$ Au electrode that were more affected by extraneous noise. The $25 \mu \mathrm{m}$ Au disk electrode was axially aligned with the outlet of the capillary and positioned 10-20 $\mu \mathrm{m}$ from the end of the capillary. Electrode-capillary separations greater than this began to broaden CE peaks while smaller separations decreased sensitivity. By monitoring the SWV voltammogram peak with the separation voltage on and off, we found that with an electrode positioned as indicated above, a shift of $\sim-250 \mathrm{mV}$ occurred in the voltammogram when a $30 \mathrm{kV}$ separation voltage was applied $(40 \mathrm{~cm} \times 25 \mu \mathrm{m}$ capillary, $50 \mathrm{mM}$, pH 3.0 phosphate buffer). To account for this, a $250 \mathrm{mV}$ offset voltage was added to the waveform applied to the working electrode during electrophoretic separations. Any waveform voltages reported here do not include this offset voltage. It should be noted that although several electrodes were used throughout this experiment, little difference was observed in their response. Typically, electrode response remained constant for several months without polishing and electrode failure was due usually to a breakage of an electrical connection within the electrode due to repeated movement of the coaxial cable connected to it. 


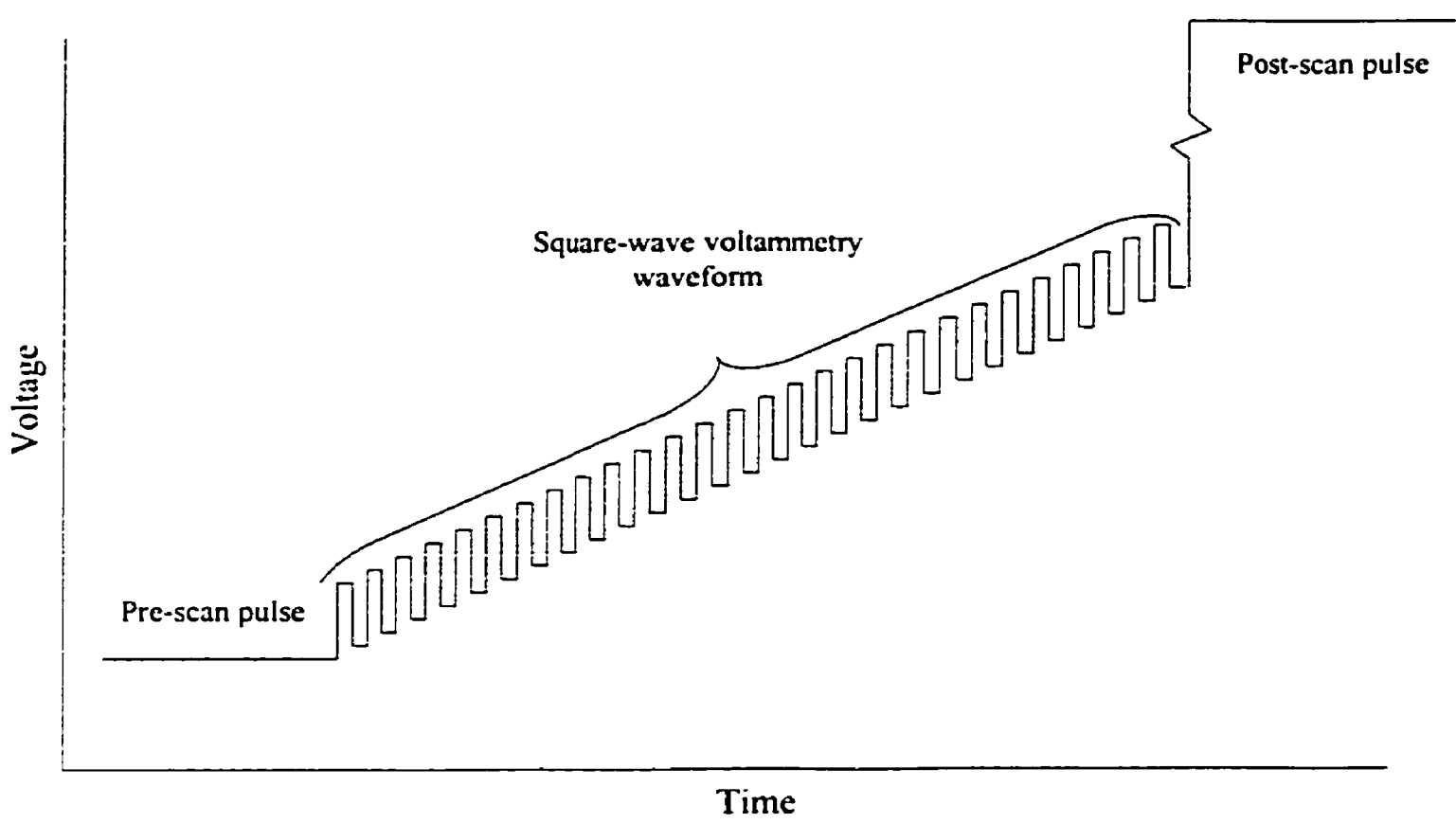

Figure 2.3 - The general SWV waveform used.

\subsubsection{Square-wave voltammetry parameters}

The general SWV waveform used is shown in Figure 2.3. The SWV parameters that could be modified were the initial/final voltages and sweep-time of the DC-ramp and square-wave amplitude and frequency. The choice of initial/final voltages was governed by the compounds being detected. It was found that for the neurotransmitters, which were oxidized at $-300 \mathrm{mV}$, a DC-ramp from 100-500 $\mathrm{mV}$ accommodated the entire SWV peak. The influence of the DC-ramp sweep-time was investigated. In the software that generated SWV waveforms, the DC ramp step height required was calculated based upon the square-wave frequency, DC ramp potential range and sweep-time that were entered. For example, a sweep-time of $100 \mathrm{~ms}$ using a square-wave frequency of $1000 \mathrm{~Hz}$ and potential range of $1000 \mathrm{mV}$ would result in the following waveform parameters: 


\section{Number of DC ramp steps: 100 \\ Step dwell time: $1 \mathrm{~ms}$ (time required to complete one $S W$ period) Step height: $10 \mathrm{mV}$}

When the scan time is reduced for a given square-wave frequency, the step height must increase and the number of steps must decrease to accommodate this. As lower scan times were used, step heights which approached the magnitude of the square-wave amplitude were reached. As a result, the forward pulse of each square-wave period was significantly higher than the reverse pulse which produced different magnitudes of charging current from the forward and reverse pulses. In such a case, charging current fluctuations were no longer compensated for adequately, and noise increased. Also, since shorter scan times result in fewer DC ramp steps (and hence fewer square-wave periods), losses in the resolution of each square-wave scan were observed. It was determined that a DC ramp time of $56 \mathrm{~ms}$ was optimal. At shorter sweep times, the SWV peak for the neurotransmitters became smaller and skewed, and $\mathrm{S} / \mathrm{N}$ decreased; longer sweep times compromised separation efficiency since they lower the overall sample rate.

A series of square-wave frequencies were examined to determine the optimal frequency for the detection of neurotransmitters. Increasing the square-wave frequency will increase the square-wave peak current, but this increase in signal will be tempered by a higher charging/faradic current ratio. The solution resistance, electrode diameter and stray capacitance of the system will limit the sensitivity gains obtained by raising the square wave frequency. As the plot in Figure 2.4 illustrates, a frequency of $2000 \mathrm{~Hz}$ was the instrumental limit for this system. Above this frequency, excessive charging currents 
interfered with the measurement of the faradic current, decreasing the dopamine $S / N$. Thus, further studies of SWV detection used a $2000 \mathrm{~Hz}$ square wave with a DC-ramp time of $56 \mathrm{~ms}$. This provided an overall sample rate of $20 \mathrm{~Hz}$ [signal averaging and pre- and post-scan pulses (discussed later in this Chapter) lowered this rate to $5-10 \mathrm{~Hz}$ ].

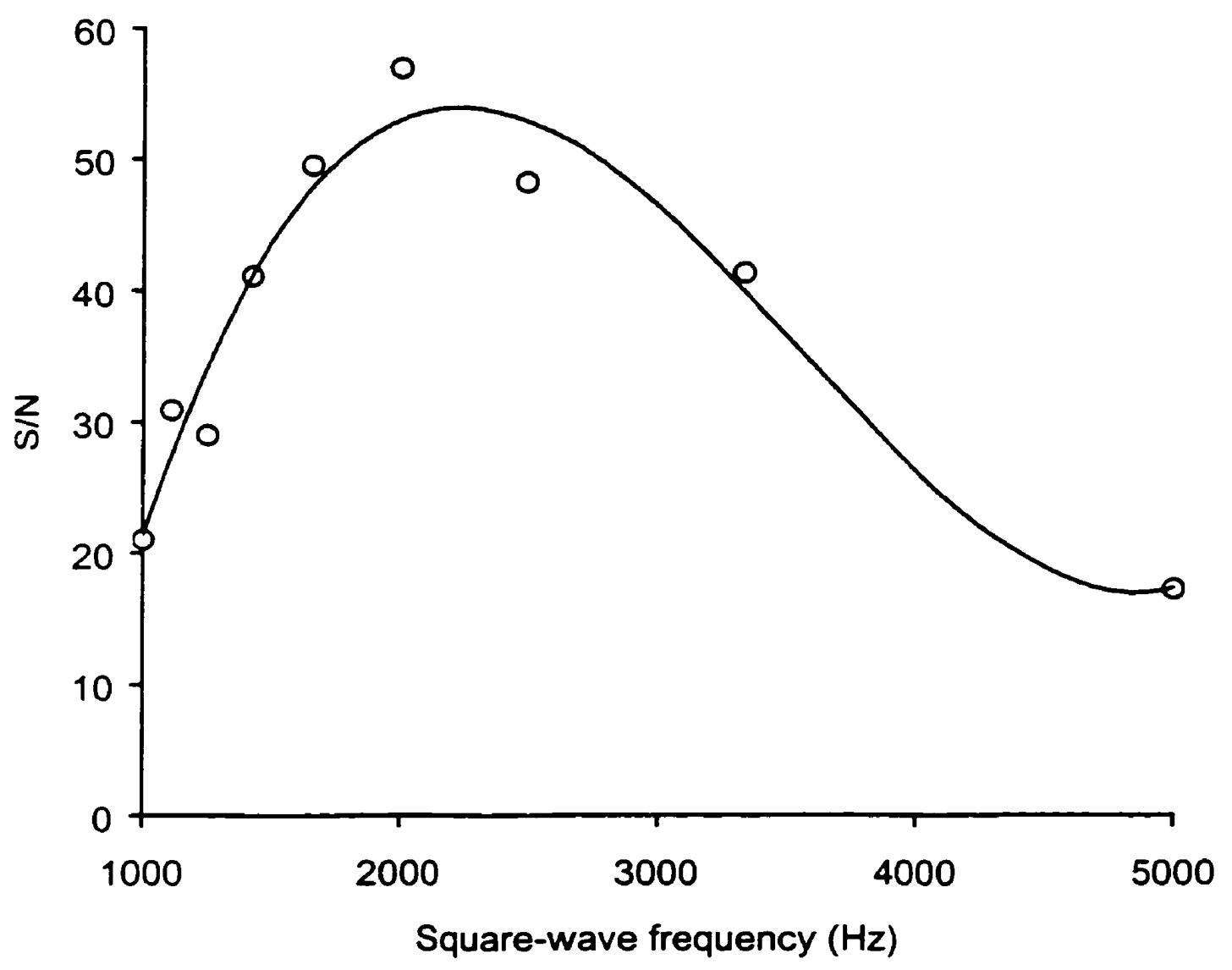

Figure 2.4 - Effect of square-wave frequency on dopamine $\mathrm{S} / \mathrm{N}$. CE conditions: $50 \mathrm{mM} \mathrm{Na} \mathrm{HPO}_{4}-$ $\mathrm{Na}_{2} \mathrm{~B}_{4} \mathrm{O}$; at $\mathrm{pH} 7.0$, I $\mu \mathrm{M}$ dopamine and epinephrine injected electrokinetically $5 \mathrm{~s} @ 10 \mathrm{kV}$. SWV conditions: 200 to $500 \mathrm{mV}$ DC ramp in $56 \mathrm{~ms}, 50 \mathrm{mV}$ square-wave, frequency varied. 


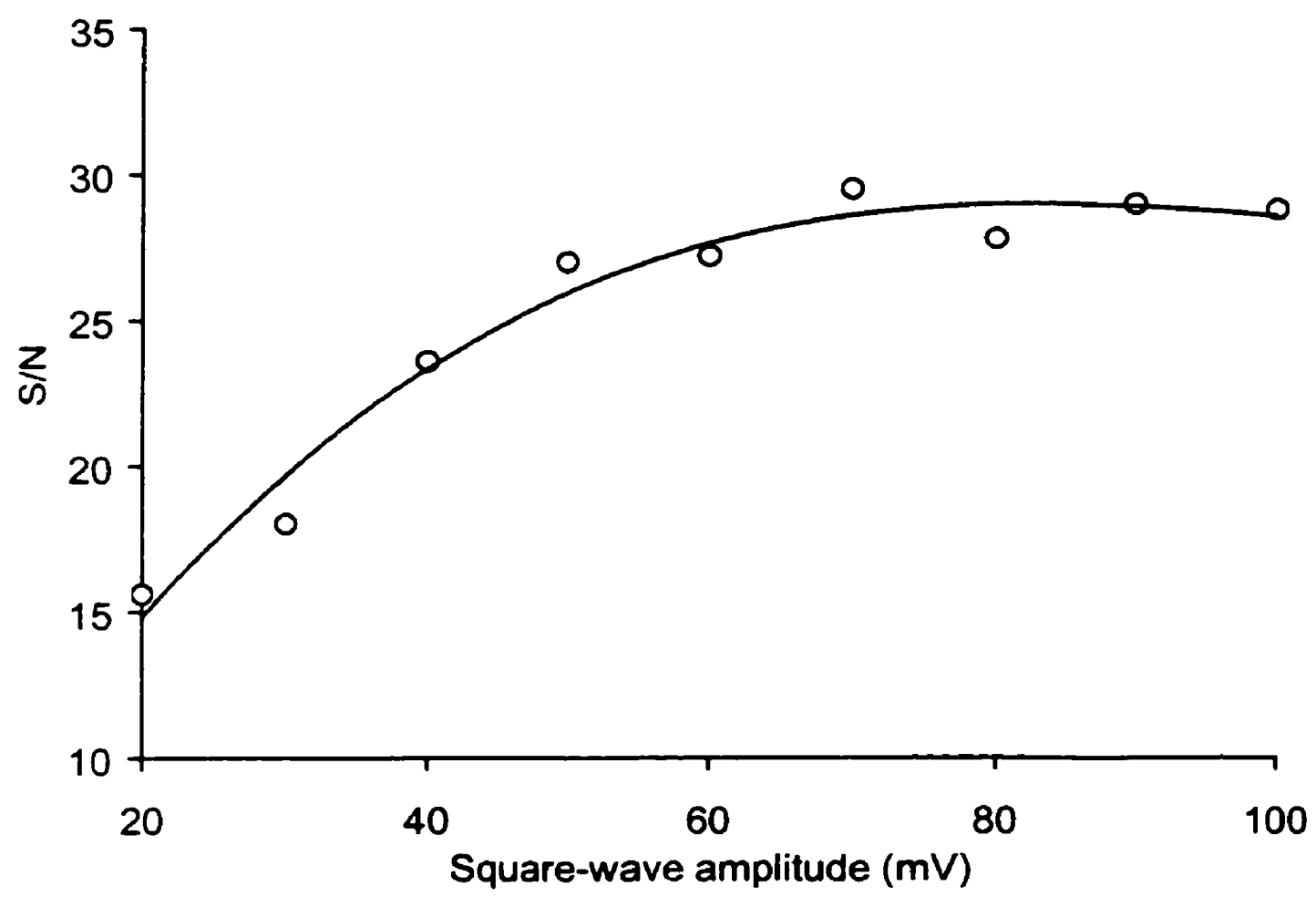

Figure 2.5-Effect of square-wave amplitude on dopamine S/N. See Figure 2.4 for conditions.

Theoretically, the optimal square wave amplitude for an reversible system is $50 / n$ $\mathrm{mV}^{45}$. To determine whether reversibility or interference from charging currents may require a different square wave amplitude for the test solutes studied, various amplitudes were investigated. As shown in Figure 2.5, increasing $\mathrm{S} / \mathrm{N}$ was observed with increasing amplitude until $\sim 60 \mathrm{mV}$ after which $\mathrm{S} / \mathrm{N}$ plateaued and then began to decrease when amplitudes greater than $\sim 100 \mathrm{mV}$ were used. Low-frequency noise (baseline drifting) was more pronounced when square wave amplitudes above $60 \mathrm{mV}$ were used. This was probably due to an increased charging/faradic current ratio making the system more sensitive to changes in the electrolyte during a $\mathrm{CE}$ run. A square-wave amplitude of 50 $\mathrm{mV}$ was found to be optimal. 


\subsubsection{SWV raw data analysis}

Having optimized the SWV parameters to minimize the charging/faradic current ratio, techniques for the interpretation of square-wave current response were investigated to maximize the $\mathrm{S} / \mathrm{N}$ where a higher than optimal charging/faradic current ratio may exist. In the traditional Osteryoung square wave method ${ }^{45}$, current is sampled at two points for each square wave, $t_{1}$ (the end of the first pulse of the square wave) and $t_{2}$ (the end of the second pulse of the square wave). The difference current [(current at $\left.t_{2}\right)-\left(\right.$ current at $\left.\left.t_{1}\right)\right]$ for each square wave is plotted versus DC ramp voltage to obtain a peak-shaped voltammogram for an electroactive species (as is illustrated in Figure 2.1d). With lower frequency square-waves, the majority of the charging current will have decayed at the end of each pulse, allowing the faradic current to be sampled independently as per the traditional Osteryoung technique. When high scan-rates are used, which require high frequency square-waves, a more flexible current sampling method is needed to maximize $\mathrm{S} / \mathrm{N}$. The software that was developed enabled sampling of the current across the entire square-wave period. The user could select the portion of the forward and reverse current response that was used to calculate the difference current. Figure 2.6 shows the impact that the amount of initial square-wave current response rejected has on $\mathrm{S} / \mathrm{N}$. 


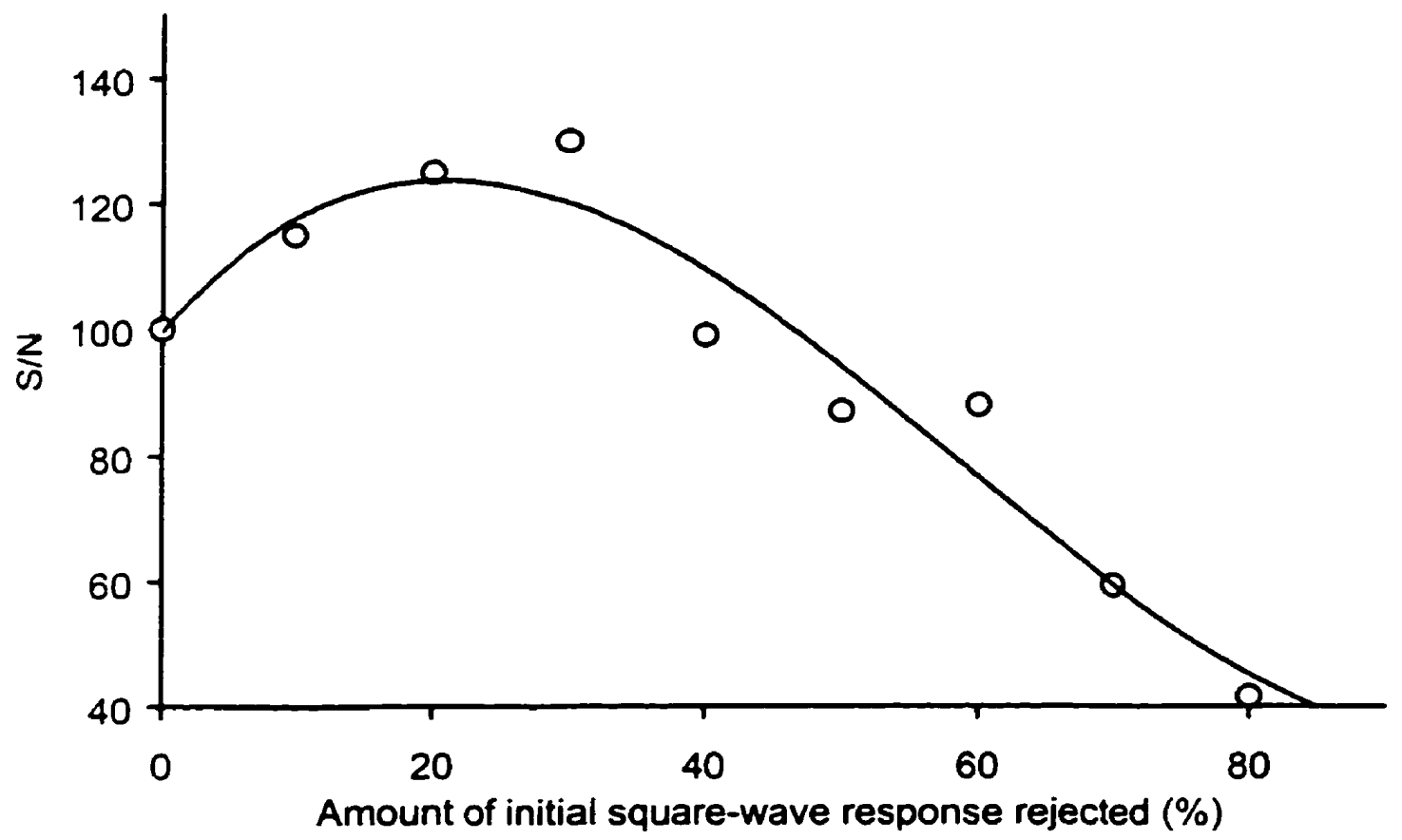

Figure 2.6 - Effect of the amount of initial square-wave current response rejected on dopamine $\mathrm{S} / \mathrm{N}$. See Figure 2.4 for conditions.

The data show that it was advantageous to collect current samples over a wide range of the forward and reverse pulses and then use signal averaging to increase the S/N. Only when almost all of the current response was used to calculate the difference current (i.e. $0-20 \%$ of the initial current rejected), was the $\mathrm{S} / \mathrm{N}$ was lower, possibly due to an increased charging component. When $>30 \%$ of the initial current response was rejected, the advantage of signal averaging was reduced and $\mathrm{S} / \mathrm{N}$ decreased. A rejection ratio of 30 $\%$ was optimal for the neurotransmitters studied. Other analyte/electrolyte systems may need to be optimized independently as the optimal rejection ratio will depend on the frequency of the square wave, conductivity of the electrolyte, diameter of the electrode 
and diffusion rate of the analyte. However this is a relatively simple process since the raw data from each electrophoretic run can be stored and reanalyzed.

\subsubsection{Obtaining quantitative information from voltammograms}

The peak height of the square-wave voltammogram (Figure 2.2) is normally plotted versus time to obtain a time-resolved quantitative plot. When this method was used for CE-SWV detection, a drifting and noisy baseline was observed due to two phenomena. Firstly, there was a general movement of the voltammogram up and down the current scale. This movement caused random noise $(\sim 0.5 \mathrm{~Hz})$. Secondly, the general shape of the voltammogram of the background electrolyte gradually changed over the course of a CE run, causing a general baseline drift. Although the exact cause of these phenomena was not investigated, it is likely that the solvent electrolysis products, created in the CE-ECD cell due to the separation current, changed the conductivity and composition of the small-volume of terminating electrolyte in the CE-ECD cell; these electrolysis products could also have been migrating past the working electrode into the separation capillary. To reduce the effects of this noise, an algorithm was developed that determined the position of the center of the voltammogram peak and drew a baseline across the bottom of the peak using a user defined peak width (see Figure 2.7). This algorithm was similar to that used in chromatographic applications where peaks are identified by scanning the data for inflection points. Using both the baseline-corrected peak height (Figure 2.7b) and the average peak current (Figure 2.7c) of the baselinecorrected voltammogram peak, drifting in the electropherogram baseline was significantly reduced for a range of peak widths The average peak current method gave the best $S / N$ 
when an optimal detection peak width of $80 \mathrm{mV}$ was chosen. Narrower peak widths did not take advantage of signal averaging while wider peak widths included weak data at the peak's edges that lowered the overall average. Although noticeable voltage shifts of voltammogram peaks were not typically observed within a run, it was not uncommon for a voltammogram peak to shift $10-20 \mathrm{mV}$ throughout a day. This algorithm was able to accommodate these shifts and give consistent quantitation without user intervention. The detection algorithm was compact enough that it could be applied in real-time rather than requiring a post-run processing step.

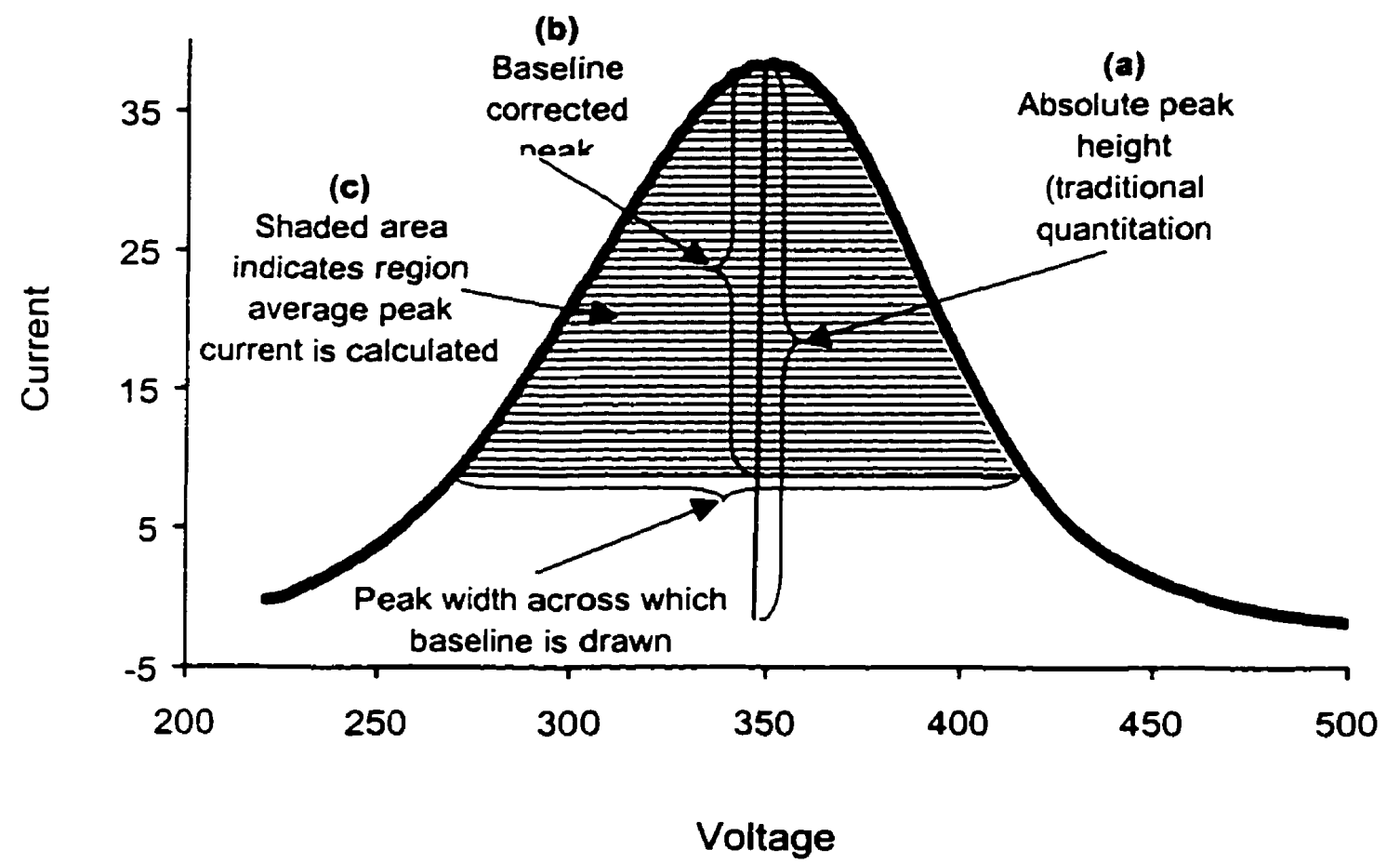

Figure 2.7 - Illustration of voltammogram quantitation techniques.

Various forms of signal averaging can be used to improve the $\mathrm{S} / \mathrm{N}$ of continuous signals. In the case of CE-SWV, where sampling periods of 50-100 ms were used, care 
had to be taken to ensure that temporal resolution losses did not compromise the separation power of the CE system. We found that a running average offered a better compromise between $\mathrm{S} / \mathrm{N}$ and peak resolution than simple signal averaging. Figure 2.8 illustrates the effect that the number of points included in the running average had on the $\mathrm{S} / \mathrm{N}$ and peak efficiency of dopamine. Peak efficiency began to decline after more than -10 points were included in the running average, while $\mathbf{S} / \mathrm{N}$ was a maximum at $\sim 20$ points. For our applications, a running average of 15 points was chosen as an appropriate compromise between peak efficiency and $\mathrm{S} / \mathrm{N}$.

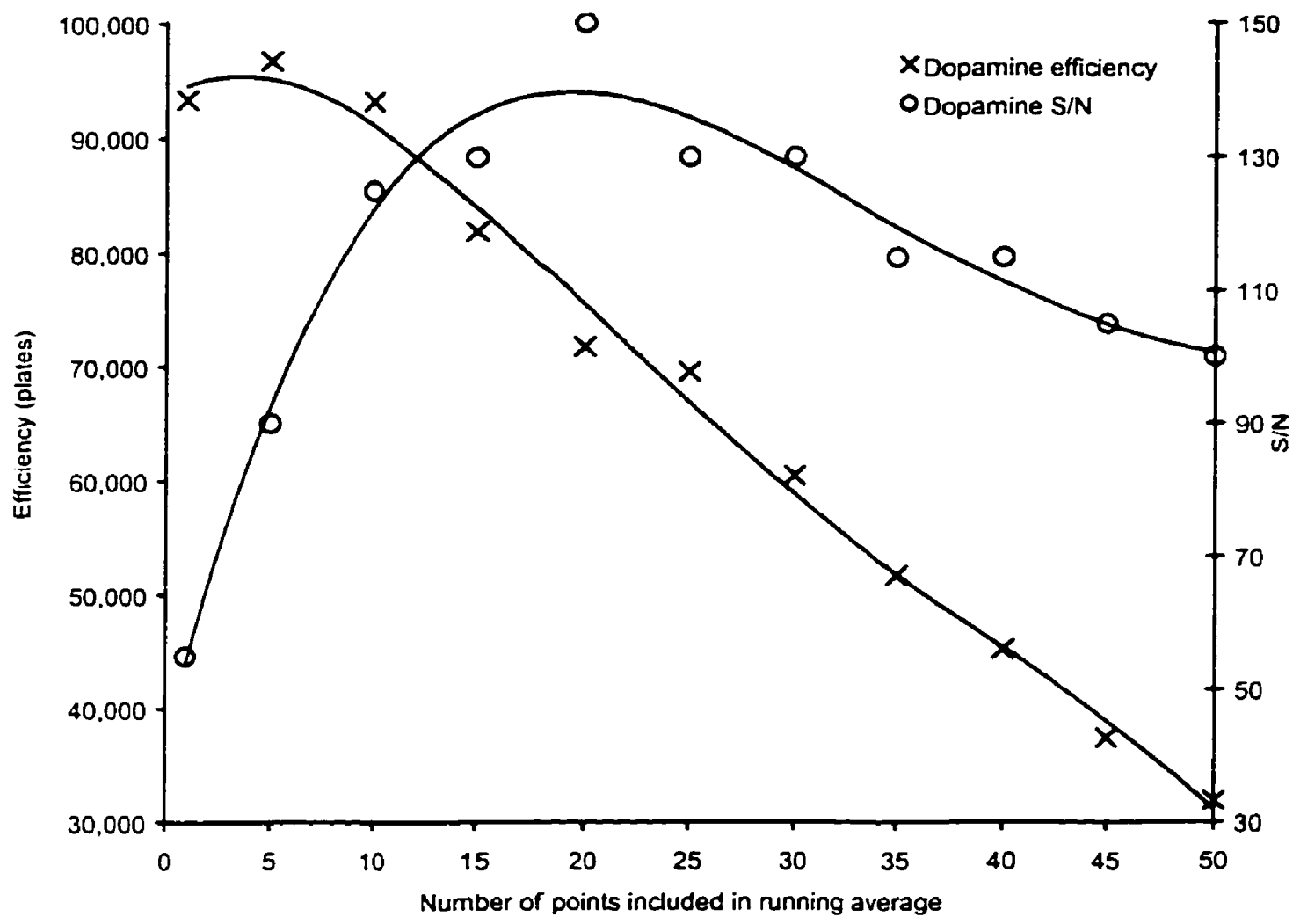

Figure 2.8 - Effect that of number of points included in the running average on $\mathrm{S} N$ and peak efficiency of dopamine. See Figure 2.4 for conditions. 


\subsubsection{Pre-and post-scan voltage pulses}

Detection performance was found to improve with the addition of pre- and postscan pulses to the applied SWV waveform. Kounaves and Young found that pretreatment of carbon-fiber electrodes by repeatedly applying a $0-1500 \mathrm{mV}$ triangular pulse for $60 \mathrm{~s}$ before use improved $\mathrm{S} / \mathrm{N}$ by a factor of 2 for SWV detection in liquid chromatography ${ }^{49}$. A significant improvement in sensitivity was obtained in the present study when the Au electrode was similarly pre-conditioned before each run. However, it was found that when a high-voltage potential pulse was incorporated at the end of the detection waveform, the run-to-run reproducibility and within-run stability of the system increased dramatically. Studies showed that a post-scan pulse voltage of $1000 \mathrm{mV}$ was found to be optimal and also that a pre-scan pulse was required to re-equilibrate the electrode after the high-voltage post-scan pulse. Although one would have expected that the re-equilibration of the electrode should be done at the initial DC-ramp potential, when pre-scan potentials from 200 to -1000 were examined, lower pre-scan equilibration voltages improved the $\mathrm{S} / \mathrm{N}$ for the detection of neurotransmitters. A pre-scan potential of $-500 \mathrm{mV}$ was found to be optimal. The exact nature of this enhancement is not fully understood. It may be that the lower pre-scan potential provided a more amenable surface for the neurotransmitters to physically adsorb and pre-concentrate, or conditioned the electrode surface to a state that is more suitable for electrochemical reaction. This more negative pre-scan potential also improved the stability of the system (less baseline drifting). Pre- and post-scan pulses of $30 \mathrm{~ms}$ duration were found to be optimal. Longer pulse times did not provide significant improvements but increased the overall scan time, thus reducing the chromatographic sample rate. 


\subsubsection{Sourier-transform filtering of voltammograms}

A Fourier transform can be used to convert time-based data to frequency-based. A filter can then be applied to eliminate particular frequency ranges, and an inverse Fourier transform of this filtered data set will return the time-based data with these frequencies eliminated. The SWV response of an electroactive analyte is of relatively low frequency compared to the noise in each SWV scan. Thus with Fourier transform filtering the majority of the higher-frequency noise should be eliminated. As Figure 2.9 illustrates, the low-frequency SWV response of dopamine could be effectively isolated from the higherfrequency electronic noise.

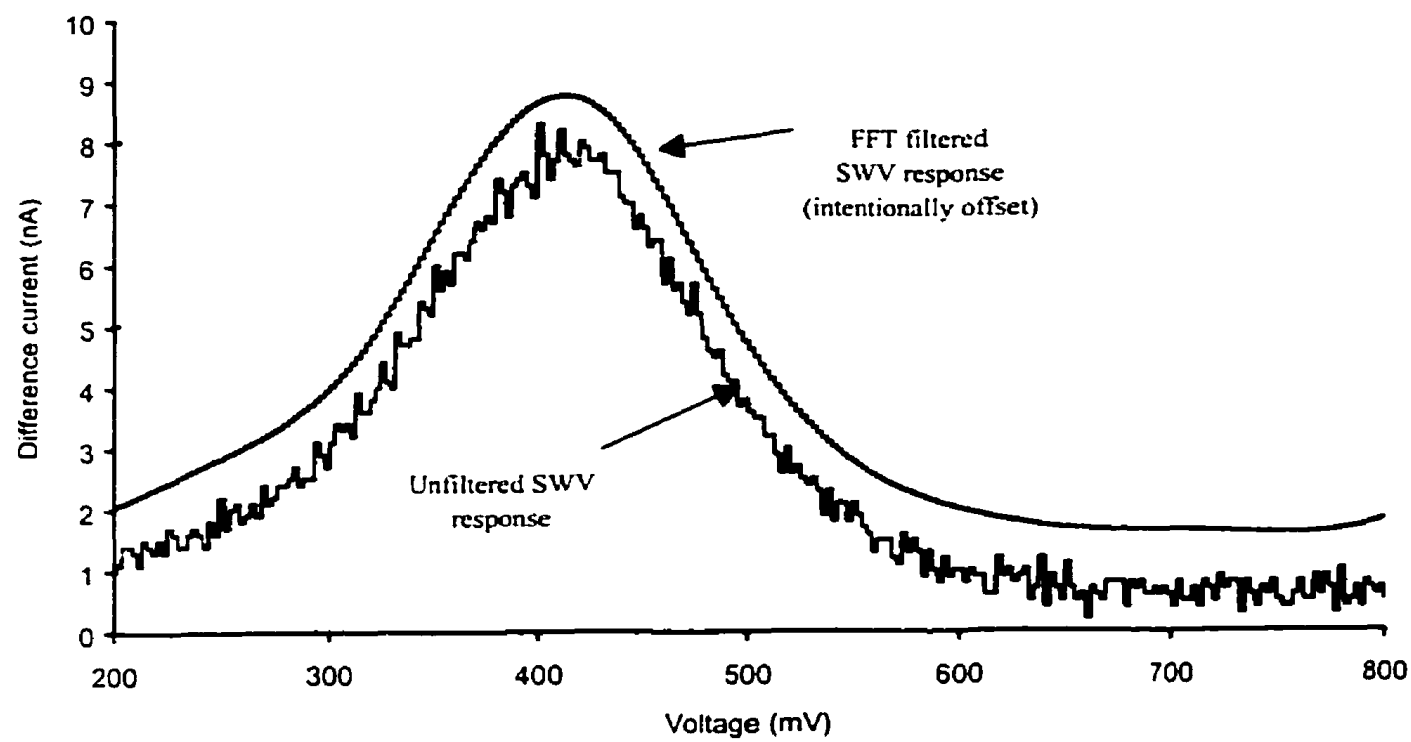

Figure 2.9 - Illustration of the impact of Fourier transform filtering on SWV. FFT filtered (upper plot) and unfiltered (lower plot) SWV voltammograms of dopamine are shown. See Figure 2.4 for conditions.

Although this type of noise elimination initially appeared impressive, when the voltammogram quantitation algorithms (section $2.3 .4, \mathrm{p} 78$ ) were applied to these filtered 
voltammograms, electropherograms with only modest improvements in $\mathrm{S} / \mathrm{N}$ were observed (Figure 2.10). This lack of significant improvement occurs because the quantitation algorithm averages the response across a rather wide time range of the SWV scan, and the resulting higher-frequency noise is reduced, as it is in Fourier transform filtering.

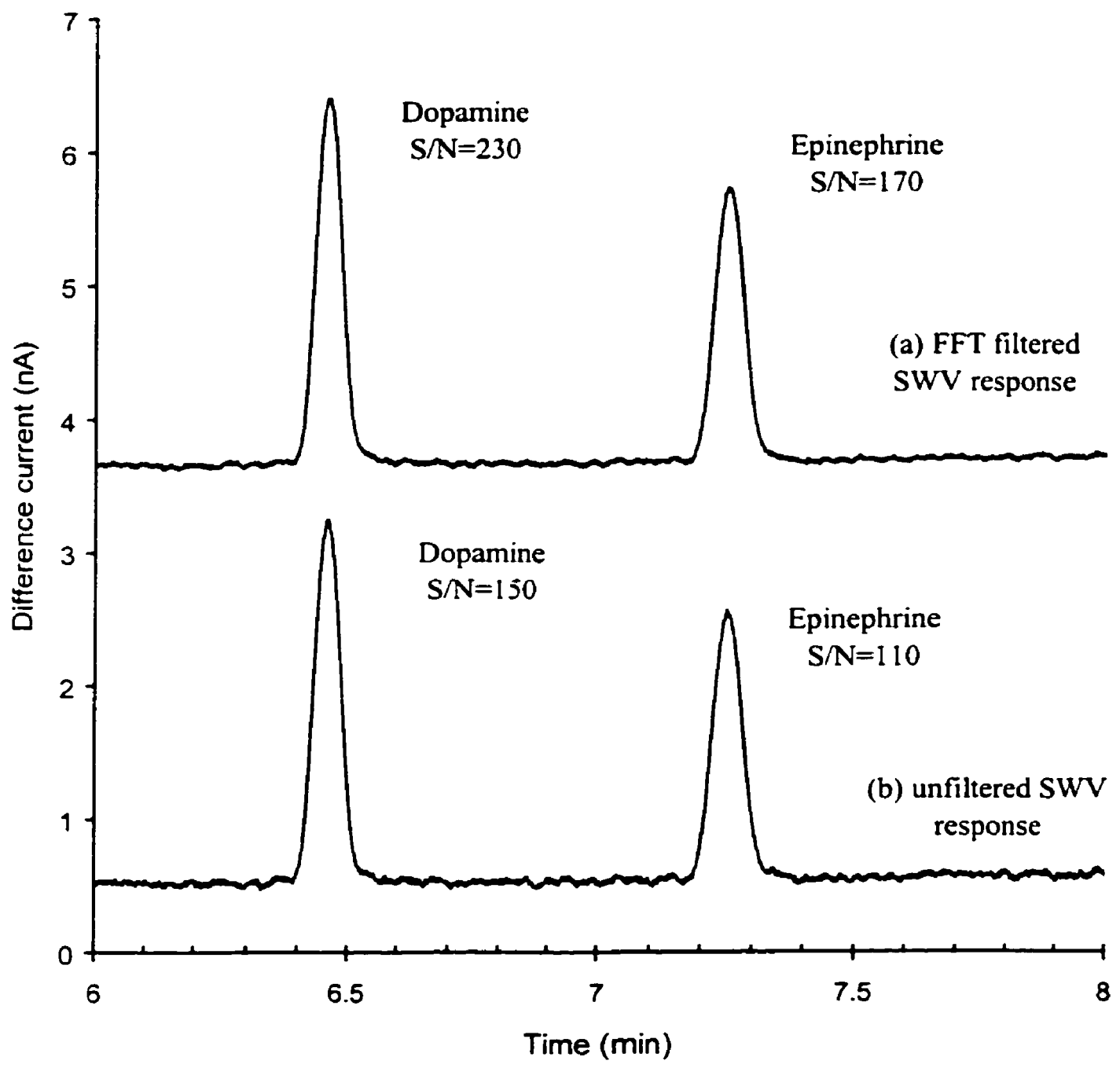

Figure 2.10 - Effect of Fourier transform filter on electropherogram noise. See Figure 2.4 for conditions. 


\subsubsection{CE-SWV of Neurotransmitters}

A CE-SWV electropherogram of dopamine and epinephrine $\left(5 \times 10^{-6} \mathrm{M}\right)$ using the optimized conditions is shown in Figure 2.11. Linear calibration curves were obtained for both neurotransmitters for concentrations in the range $0.50-50 \mu \mathrm{M}$ (Figure 2.12).

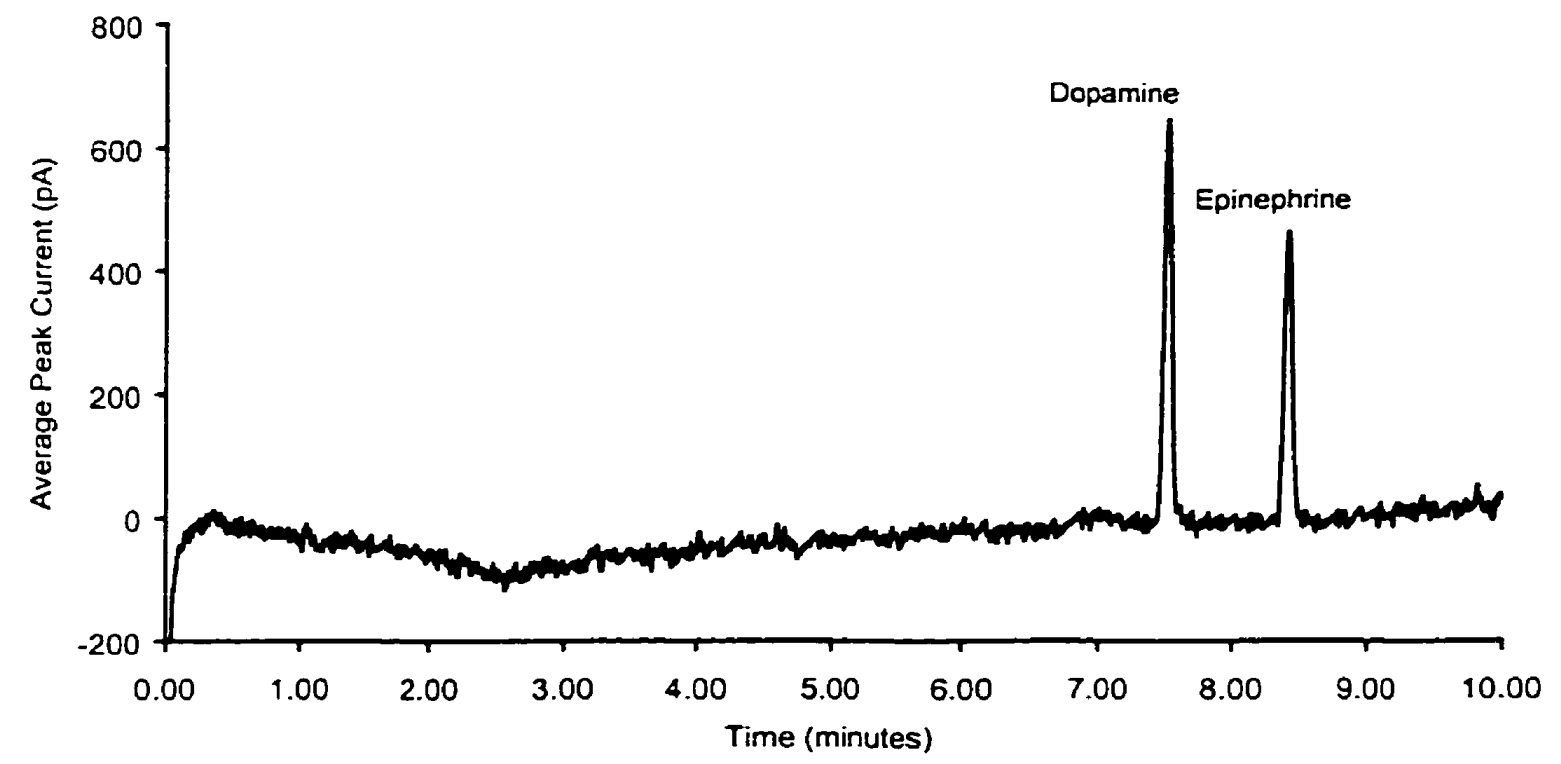

Figure 2.11-CE-SWV electropherogram of $5 \mu \mathrm{M}$ dopamine and epinephrine. See Figure 2.4 for conditions.

Because the current follower gain was optimized for maximum sensitivity, neurotransmitter concentrations above $-70 \mu \mathrm{M}$ did not give a linear response. The linear range of the system could be moved higher (sacrificing sensitivity) by lowering the gain of the current follower. Although the correlation coefficient for the calibration curve was good, this is often a poor indicator of the linearity of a calibration curve ${ }^{50}$. By plotting the response factor (peak area/dopamine concentration) versus dopamine concentration, reasonable linearity $( \pm 20 \%$ ) was observed only in the 5-50 $\mu \mathrm{M}$ region (Figure 2.13 ). Although this linear region is quite narrow, that it is not atypical to find calibration curves 
with good correlation coefficients to have more limited linearity when the response factors are plotted $^{50}$. The source of this nonlinearity may be the use of the electrokinetic injection method, as the amount of analyte injected will depend upon the conductivity of the injection solution (which changes slightly with decreasing analyte concentration). Use of an internal standard may eliminate this nonlinearity (this was investigated in Chapter 3). A detection limit of $150 \mathrm{nM}(1.5 \mathrm{fmol})$ for dopamine using CE-SWV was determined by injecting successively lower concentrations until a $\mathrm{S} / \mathrm{N}$ of 3 was obtained. This detection limit compares favorably to detection limits of CE separations of neurotransmitters obtained using constant potential amperometric detection by Zhong et al [ $180 \mathrm{nM}, \mathrm{S} / \mathrm{N}=3$ $(120 \mathrm{nM}, \mathrm{S} / \mathrm{N}=2$ reported $)]^{51}$, Hu et al $(20 \mathrm{nM}, \mathrm{S} / \mathrm{N}=3)^{22}$ and Ewing et al (0.2-0.4 fmol, $\mathrm{S} / \mathrm{N}=3$, extrapolated from high concentration injections) $)^{13}$.

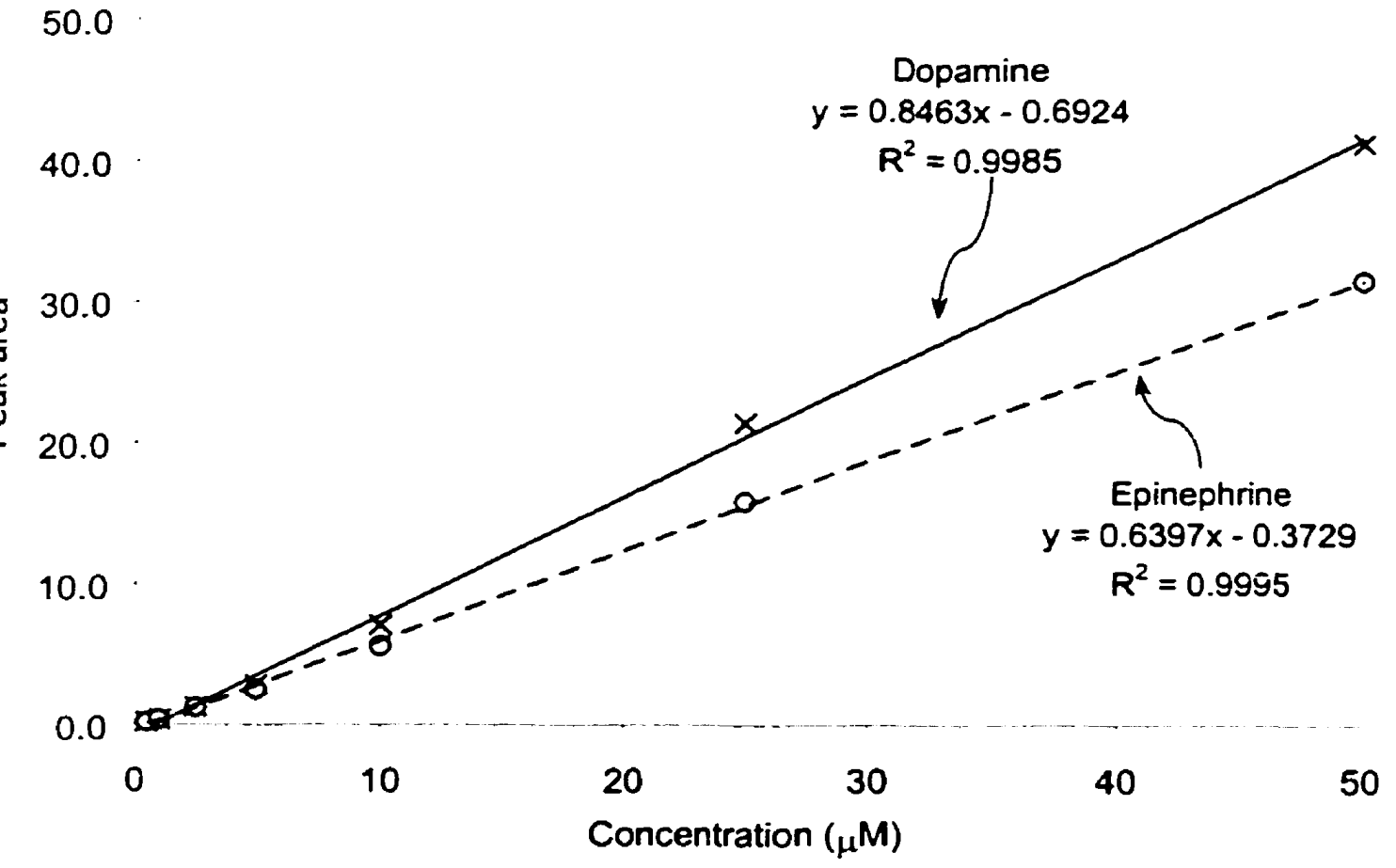

Figure 2.12 - Calibration curves for dopamine and epinephrine. 


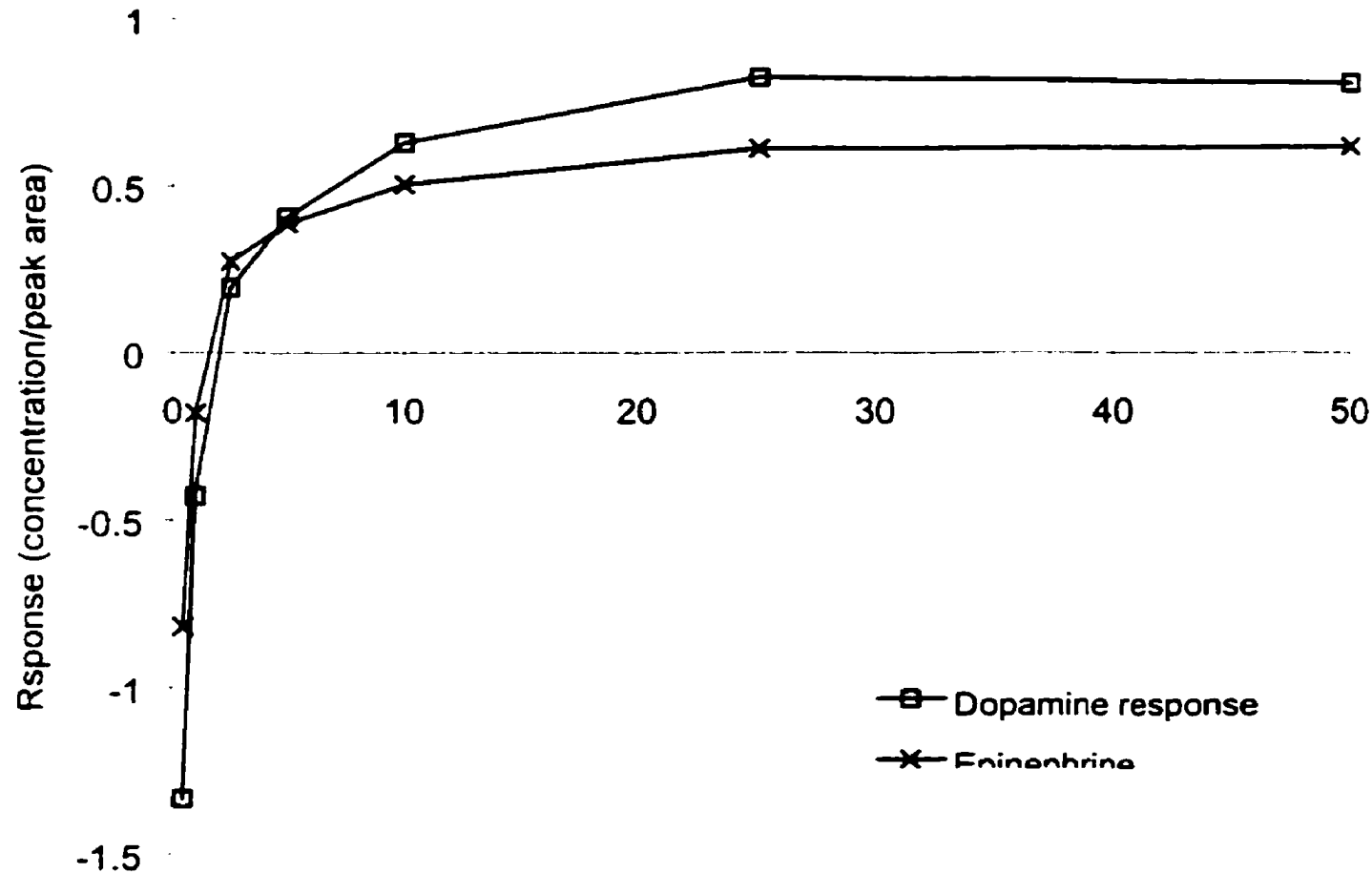

Concentration $(\mu \mathrm{M})$

Figure 2.13 - Sensitivity plots for dopamine and epinephrine.

A more qualitative illustration of the CE-SWV data is shown in a 3-D contour plot (Figure 2.14). A comparison of Figure 2.11 and Figure 2.14 illustrates the extent to which baseline drift can be eliminated using the quantitation techniques discussed above. The electropherogram shown in Figure 2.11 has only minimal baseline drift while the 3-D contour plot (Figure 2.14) shows a large initial baseline drift at potentials near the peak voltage of the neurotransmitters (note the difference in current scale between Figure 2.11 and Figure 2.14). This 3-D contour plot also illustrates the potential for analyte identification and calculation of peak purity based on an analyte's SWV response. 


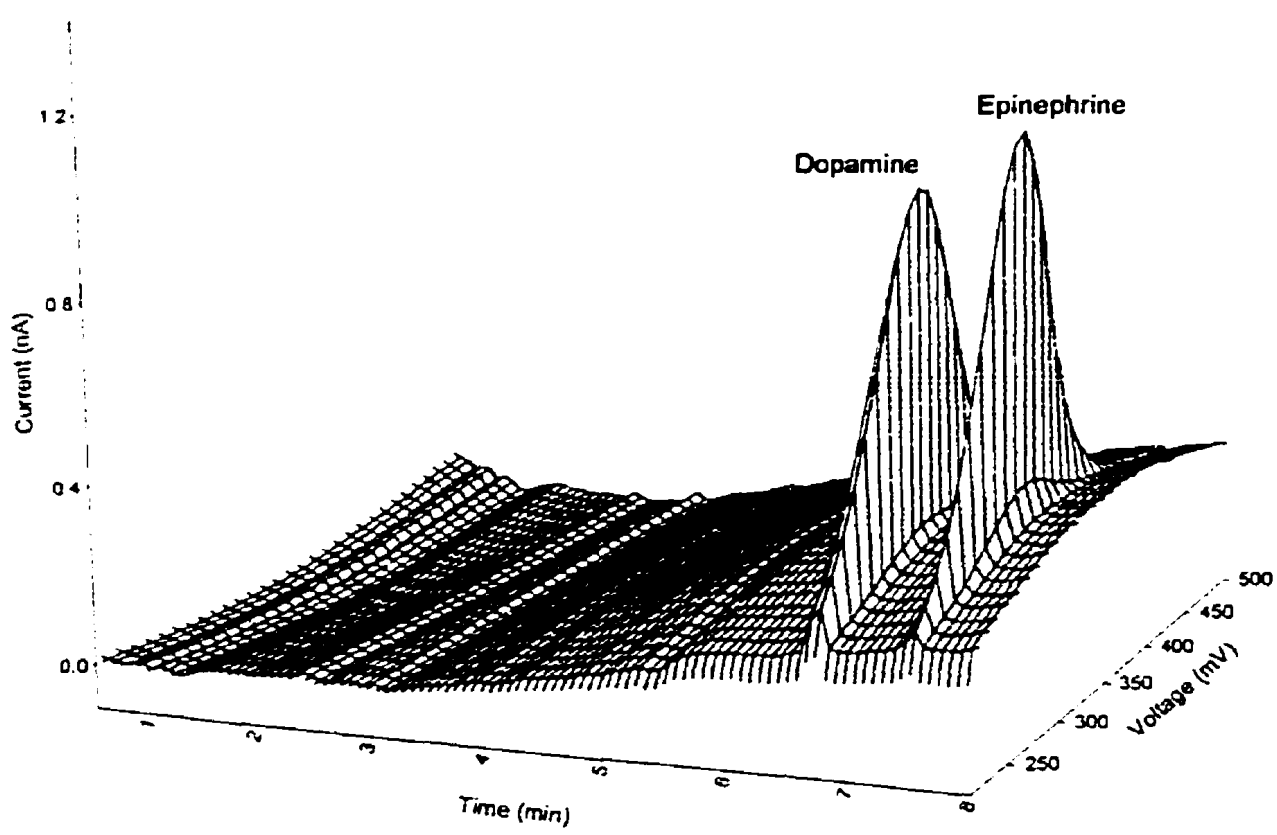

Figure 2.14-3-D plot of $10 \mu \mathrm{M}$ dopamine and epinephrine separation. See Figure 2.4 for conditions.

\subsection{Conclusion}

Previously, to obtain the sensitivities for CE-ECD reported here, constant potential amperometry using complex decoupling systems and cylindrical carbon fiber electrodes were used. This technique, because of the fragility of the electrode/decoupling system and the potential for electrode fouling in a non-pulsed system, makes it unsuitable for routine use. Conversely, the CE-ECD system described here is both sensitive and robust. While previous reports of voltammetric detection have been unable to provide sampling frequencies demanded by $\mathrm{CE}$, the SWV method reported here allowed sample rates of 5$10 \mathrm{~Hz}$, adequate for most CE separations. A potential limitation of CE-SWV may be that, 
due to its high frequency $\mathrm{AC}$ waveform, only compounds with reversible electrochemical kinetics will respond. Unlike simpler electrochemical techniques such as amperometric detection, SWV is capable of investigating other non-faradic electrode surface processes such as analyte adsorption. In Chapter 3, CE-SWV will be used to monitor analyte adsorption in an effort to develop a more generally applicable CE detection method. 


\section{CHAPTER 3 - ADSORPTION-BASED ELECTROCHEMICAL DETECTION}

\subsection{Introduction}

Although Chapters 1 and 2 described the development of a sensitive and robust CE-SWV system, it was applicable only to electrochemically active analytes. Since the goal of this research was to develop a more generally applicable sensitive detection method, further work was required to expand the applicability of this system. One of the reasons a SWV detection system was investigated is that it has the possibility of more general applicability. Unlike other electrochemical detection approaches CE, SWV is not limited to investigating simple redox processes. Physical chemists often use AC techniques such as SWV to investigate other electrode surface phenomena such as the adsorption of molecules onto an electrode surface. Unlike redox reactions, adsorption is a much more general phenomenon. Although adsorption will depend on the structure of the molecule, adsorption onto metal electrodes will occur in varying degrees with most organic compounds ${ }^{52}$. The nature of the resulting change in electrode response will depend on a variety of parameters.

The adsorption of organic molecules onto a metal electrode can occur as either physical or chemical adsorption (chemisorption). In an aqueous environment, physical adsorption of organic molecules onto an electrode surface will occur due to hydrophobic effects. Organic analytes lacking sufficient hydrogen-bonding sites will be excluded from the highly ordered water matrix existing in the bulk solution. A polarized electrode will 
have a layer of water molecules (their dipoles attracted to the electrode charge) as well as counter ions on its surface. The potential of an electrode can be adjusted, such that its surface has little or no charge, so that water molecules or ions will have a lesser attraction to its surface. It is at this potential that physical adsorption of organic analytes is favoured as the competition between these analytes and adsorbed water molecules and ions is minimized. It has been shown that in a potential range between $\sim-500$ to $-1600 \mathrm{mV}$ organic analytes adsorb onto a $\mathrm{Hg}$ electrode (1 $\mathrm{M} \mathrm{Na}_{2} \mathrm{SO}_{4}$ electrolyte); outside of this potential region capacitance measurements indicated that the electrode was free of adsorbed organic material ${ }^{53}$. This study was encouraging as it indicated that while the potential region suitable for analyte adsorption is quite wide, there are potential regions that can be used to eliminate adsorbed organic material from the electrode surface (necessary in a flowing system to maintain a clean electrode surface). Baranski and Norouzi have demonstrated that an adsorption-based detection method can be used for a flow injection system ${ }^{5+}$. They measured the interference of the background cyclic voltammetry response when an analyte interacted with a Au or Pt electrode. For a variety of organic analytes, the background response was either reduced or voltammogram peaks were shifted to more extreme potentials as the analytes interacted with the electrode, indicating that physical adsorption was responsible for the response.

Chemisorption, in contrast to physical adsorption, is a process which involves chemical bonding of the adsorbing molecule with the electrode. Organic molecules with "available" electron density (i.e. bonding electrons stabilized by $\pi$-resonance structures, or non-bonding valence electron pairs) can be used to form covalent bonds with noble metal 
electrodes such as $\mathrm{Pt}$ and $\mathrm{Pd}$ that have unfilled $d$-orbitals which can accept this electron density. As might be expected, adsorption of this type has been shown to be irreversible ${ }^{55}$. Chemisorption, although not a typical redox process, will also produce a current response as it does involve a change in the electronic structure of the electrode. As with physical adsorption, the presence of the adsorbed species on the electrode surface will also change the background current response normally observed for a clean electrode surface. Baranski et al, investigated the waveform frequency dependence of the adsorption of methionine onto a $\mathrm{Pt} \mu \mathrm{m}$-electrode in a flow-injection system to determine the nature of the adsorption mechanism ${ }^{56}$. At high frequencies $(-5 \mathrm{MHz})$, a decrease in the electrode response was observed when methionine passed by the electrode, while at more moderate waveform frequencies $(15-300 \mathrm{kHz})$, an increase in the electrode response was observed, that was attributed to methionine decomposition. This study not only verified the complexity of the adsorption process, but also introduced the possibility of monitoring complex analyte adsorption processes using $\mathrm{AC}$ waveforms to obtain an analytical signal.

SWV was originally developed as a technique for discrimination between charging currents and faradic currents ${ }^{45}$. The SWV system that was developed during this research (Appendix A) allows the adjustment of current sampling and waveform parameters to values outside those normally used to investigate reversible faradic processes such as the oxidation of neurotransmitters discussed in Chapter 2. Thus CE-SWV should permit measurement of electrode-surface processes such as physical/chemisorption. In addition, the presence of organic molecules in the diffusion layer may also result in a change in the conductivity of the diffusion layer which will affect both the potential shift at the electrode 
due to an $i \mathrm{R}$ change (discussed in section 1.3.3, $\mathrm{p} 34$ ) and the charging current response of the electrode to an alternating potential (discussed in section 2.3.2, p 72). These conductivity induced effects will also produce a change in the normal background SWV response. All of these effects may result in a detection method that is applicable to a wide range of analytes.

The measurement of the physical adsorption of organic analytes onto a Pt electrode by Baranski and Norouzi used cyclic voltammetry $(\mathrm{CV})^{54}$. Although at sufficiently high scan rates $\mathrm{CV}$ can approximate an $\mathrm{AC}$ voltammetric technique and thus be used to investigate electrode surface phenomena such as physical adsorption, SWV may be a more appropriate technique. This is because in SWV, the potential dependence of analyte adsorption may be more clearly characterized and provide better sensitivity. In SWV, the current response to rapid altemating potentials are recorded as the potential is scanned linearly. However $\mathrm{CV}$, which uses a linear DC scan, is not as sensitive to the potential dependence of changes that occur in the double-layer. Using the modified Osteryoung SWV technique that was discussed in Chapter 2, these current responses (in response to an alternating potential) can be examined to determine the time dependence of the change in the current response due to analyte adsorption. By modifying the square-wave amplitude and frequency and selecting only specific time windows of the square-wave current response, the adsorption-based response can be separated from other non-specific responses more effectively than is possible using $\mathrm{CV}$ to maximize the $\mathrm{S} / \mathrm{N}$. 
This chapter will report the development of an adsorption-based electrochemical detection system for CE using SWV. The adsorption-based ECD (AdsECD) method that resulted from this investigation was applied to a wide variety of analytes separated by $\mathrm{CE}$ to both demonstrate its general applicability as well as to further investigate the types of functional groups which illicit an analytically significant response. The identification of important experimental parameters was also determined via separations performed with a variety of electrolytes of different ionic strength and $\mathrm{pH}$, organic additives, and micellar systems. For purposes of comparison, the sensitivities and peak shapes obtained with CEAdsECD were compared with those obtained with CE-UV detection.

\subsection{Experimental}

\subsubsection{Chemicals}

All solutions were prepared from water purified using an ULTROpure with a TFM membrane and NANOpure II with Type 1 ORGANICfree cartridge kit (Barnstead Corp., Dubuque, LA). Unless otherwise stated, all reagents were analytical grade. DL-Amino acids, dihydrostreptomycin, neomycin, erythromycin, oleandomycin, trimethoprim (TR), sulfamethazine (SMZ), sulfapyridine (SPY), sulfadimethoxine (SDM), sulfathiazole and penicillin V/G were obtained from Sigma-Aldrich Canada Limited (Oakville, ON, Canada). N-(2-Heptyl)-N-methylpropargylamine (2-HMP) and Deprenyl were obtained from the Department of Neuropsychiatric Research, University of Saskatchewan (Saskatoon, SK, Canada). The mixture of basic drugs used in Section 3.3.5.1 was a gift from the R.C.M.P. Forensic Laboratory (Regina, SK, Canada). The peptide mixture used in Section 3.3.5.5 which was obtained from Sigma-Aldrich Canada Limited, was a 
lyophilized mixed standard (P-2693). The nonsteroidal anti-inflamatory drugs phenylbutazone, oxyphenbutazone and diclofenac were obtained from Sigma-Aldrich Canada Limited and flunixin was obtained from Schering Canada Inc. (Point Claire, Quebec, Canada).

\subsubsection{Automated CE-ECD system}

Sections 1.2.2-1.2.6 describe the automated CE-ECD system used. Appendix A describes the data acquisition system used in more detail. Unless otherwise stated, a 25 $\mu \mathrm{m}$ Pt disk electrode prepared as described in section 1.2.2 was used in the CE-ECD cell.

\subsubsection{Commercial CE-UV system}

A Beckman P/ACE 5500 commercial CE system (Beckman Coulter, Fullerton, CA, U.S.A.) was used to generate CE-UV electropherograms shown in this Chapter. The following conditions were used for CE-UV separations: $75 \mu \mathrm{m} \times 47 \mathrm{~cm}(39.4 \mathrm{~cm}$ to detector window) fused-silica capillary, $195 \mathrm{~nm} \mathrm{UV}$ detection wavelength, $15 \mathrm{kV}$ separation potential. Electrolyte and injection conditions used for the various separations are indicated in figure captions.

\subsubsection{CE Conditions}

Fused-silica capillaries ( $25 \mu \mathrm{m}$ i.d., $365 \mu \mathrm{m}$ o.d., Polymicro Technology, Phoenix, AZ) were used in these experiments. Before use each day, capillaries were rinsed with 0.1 M potassium hydroxide for $5 \mathrm{~min}$ followed by the separation electrolyte for $5 \mathrm{~min}$. Also, before each injection, the capillary and CE-ECD cell were rinsed with $\sim 2-5$ void volumes of the separation electrolyte. All sample injections were made electrokinetically. Unless 
otherwise stated, samples were diluted to the desired concentration in the CE separation electrolyte.

A separation of sulfonamide antibiotics was used for an initial investigation of $\mathrm{CE}$ AdsECD. Sulfonamides were separated using a $100 \mathrm{mM}, \mathrm{pH} 7.0$ phosphate electrolyte, $25 \mu \mathrm{m} \times 30 \mathrm{~cm}$ capillary and $10 \mathrm{kV}$ separation potential.

The following SWV waveform and data acquisition parameters were determined to be optimal: DC voltage ramp : -600 to $-1100 \mathrm{mV}$ in $100 \mathrm{~ms}$; Square-wave frequency/amplitude: $1000 \mathrm{~Hz} / 100 \mathrm{mV} ; 0 \%$ of initial square-wave current response rejected, $3 \mathrm{SWV}$ scans included in running average. Unless otherwise stated, all CEAdsECD separations shown in this Chapter were performed using these SWV parameters.

\subsubsection{Calculations of $S / N$ and peak efficiency}

See section 1.2.8.

\subsection{Results and discussion}

\subsubsection{CE-SWV detection of adsorption-based responses for organic analytes} Initially, in an effort to develop an electrochemical detection method for CE that was responsive to non-electrochemically active analytes, the adsorption of organic analytes onto a platinum electrode was monitored. These studies showed two main patterns of response that are illustrated by the results obtained with dihydrostreptomycin and sulfonamide antibiotics. These results will be discussed first in this chapter to provide the information required for a discussion of the effect of the shape of the SWV waveform, its optimization, and the possible mechanisms responsible for the mode of electrochemical 
detection. Results obtained for several different classes of compounds will then be discussed later in this chapter.

The background SWV response observed for a $50 \mathrm{mM}, \mathrm{pH} 2.5$ separation electrolyte is shown in Figure 3.2a. This was the typical response obtained during a CE separation with no analyte zones exiting the capillary. The peaks seen in Figure $3.2 \mathrm{a}$ are likely due to hydrogen adsorption/reduction which is a complex, multistep process on a $\mathrm{Pt}$ electrode. When dihydrostreptomycin, an aminoglycoside antibiotic (structure shown in Figure 3.1), was injected and eventually exited the capillary, the electrode response changed even though the analyte does not show conventional electrochemical activity at a Pt electrode. The effect of its adsorption on the current response is shown in Figure 3.2b.

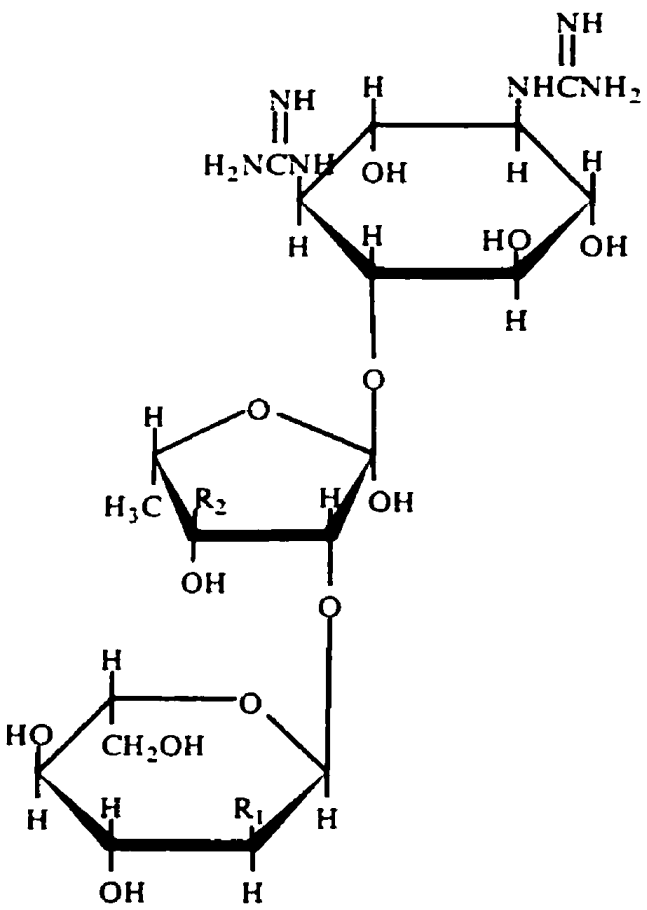

Figure 3.1 - Structure of dihydrostreptomycin. 


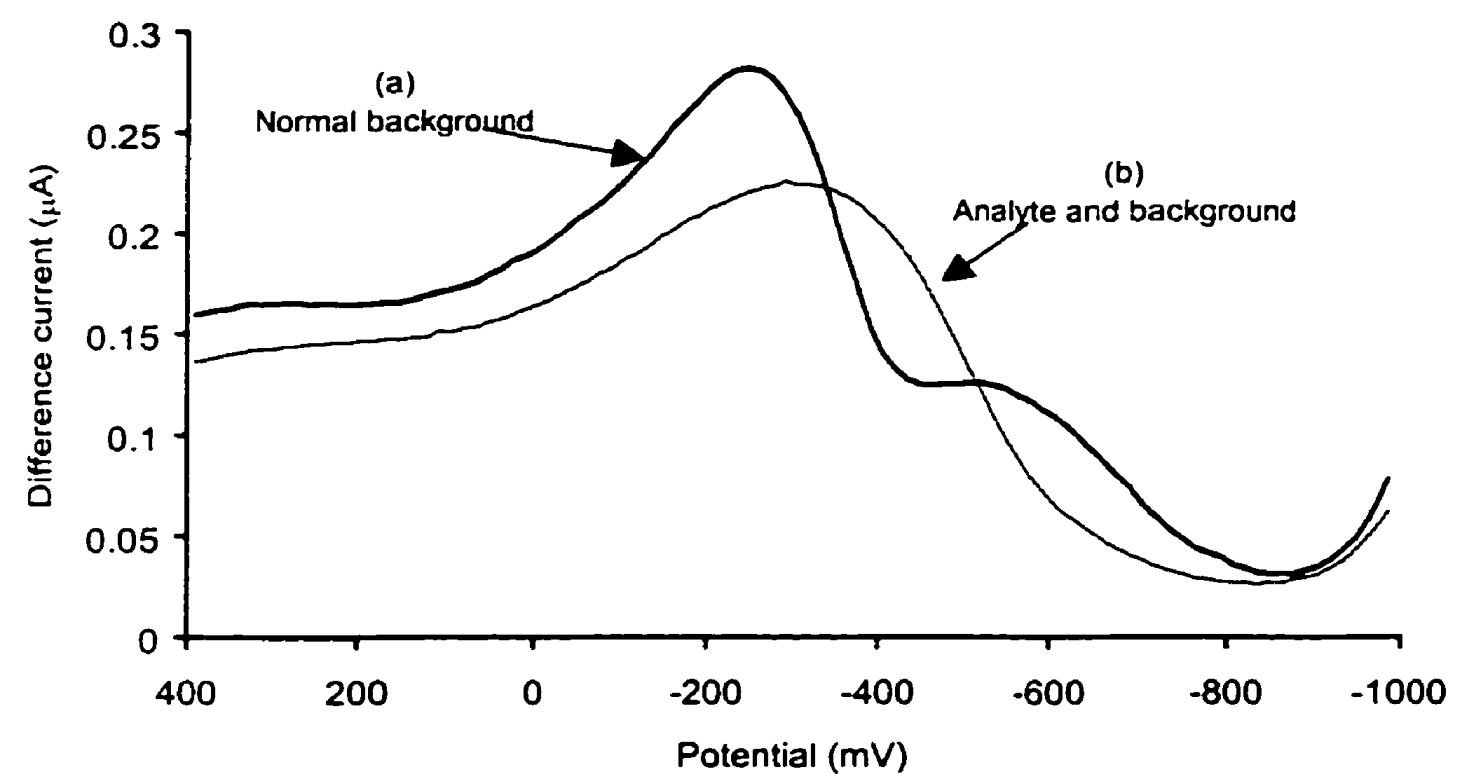

Figure 3.2 - Background SWV response for $50 \mathrm{mM}, \mathrm{pH} 2.5$ phosphate buffer (a); (a) with dihydrostreptomycin sample zone exiting the capillary (b). SWV conditions: DC voltage ramp : -1000 to $0 \mathrm{mV} / 100 \mathrm{~ms}$; Square-wave frequency/amplitude: $1000 \mathrm{~Hz} / 50 \mathrm{mV} ; 20 \%$ of initial square-wave current response rejected.

As can be seen, the normal background SWV response is reduced and the major voltammogram peak at $\sim-250 \mathrm{mV}$ is shifted slightly to more negative potentials. Unlike in the SWV detection of oxidized analytes described in Chapter 2, a peak-shaped voltammogram was not obtained and the quantitation algorithms described in Section 2.3.4 could not be used. Instead, a section of the SWV response was selected, and the response measured in this potential window was averaged and plotted versus separation time to obtain an electropherogram (see Figure 3.3). 


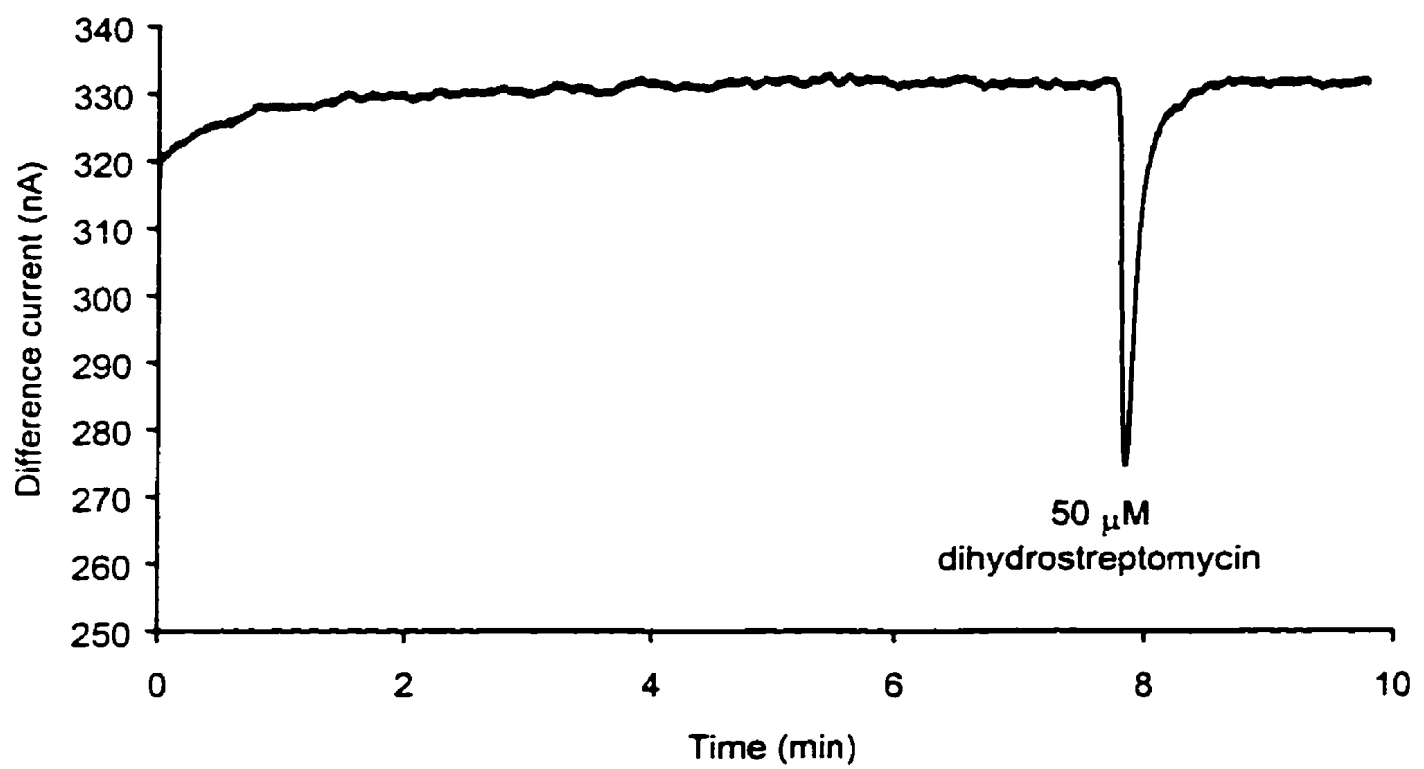

Figure 3.3 - Electropherogram of a dihydrostreptomycin CE separation. CE conditions: $50 \mathrm{mM}, \mathrm{pH} 2.5$ phosphate buffer; $50 \mu \mathrm{M}$ dihydrostreptomycin injected electrokinetically, $1 \mathrm{~s} @ 10 \mathrm{kV}$. For SWV conditions see Figure 3.2.

The electropherogram shown in Figure 3.3 demonstrates that reasonably sensitive detection limits $(\sim 1 \mu \mathrm{M}, \mathrm{S} / \mathrm{N}=3$, estimated from the separation shown in Figure 3.3) could be obtained by monitoring the effect of physical adsorption. Dihydrostreptomycin, due to its lack of a chromophore can not be detected using UV detection. For sensitive detection in HPLC systems, it is usually derivatized with a fluorescing moiety ${ }^{57}$. Other aminoglycoside antibiotics sharing similar non-aromatic, sugar-based molecular structures as dihydrostreptomycin (neomycin, erythromycin and oleandomycin) were found to give similar results and clearly show the attractive features of this detection approach for analytes that exhibit poor detection via other approaches. 
To examine the response produced by different analytes, a mixture of sulfonamide antibiotics (structures of these compounds are shown in Figure 3.4) were separated and detected by SWV.

\section{Sulfonamides}<smiles>[R]NS(=O)(=O)c1ccc(N)cc1</smiles><smiles>COc1cc(Cc2cnc(N)nc2N)cc(OC)c1OC</smiles>

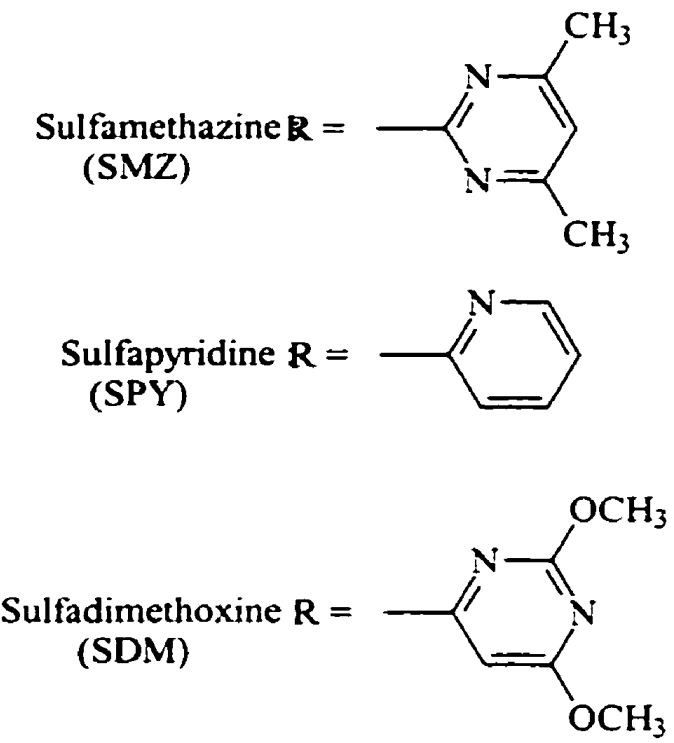

Figure 3.4 - Structures of the sulfonamide antibiotics and trimethoprim.

These analytes were selected because they are an important group of antibiotics used to combat a variety of bacterial infections in both humans and animals. All of these analytes also exhibit good UV-detection, which permitted a direct comparison of 
adsorption detection with well established UV-detection approaches in CE. Sensitive methods of analysis for these compounds are necessary to quantitate residue levels in meat for food safety considerations. Although trimethoprim (also shown in Figure 3.4) is not considered a sulfonamide antibiotic, it is commonly used in conjunction with these drugs in veterinary applications.

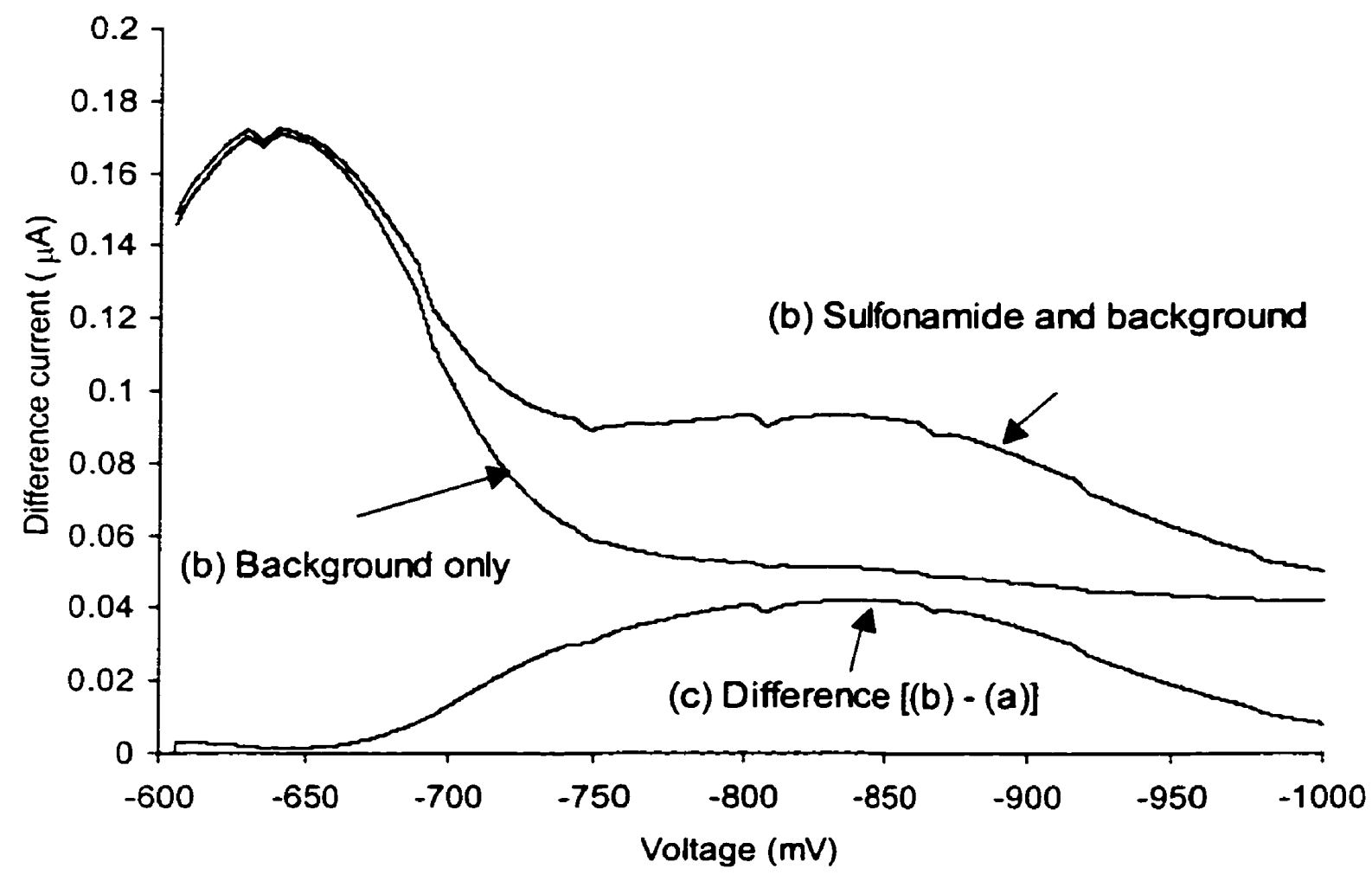

Figure 3.5 - SWV response of background electrolyte (100 mM, pH 7.0 phosphate) (a) and with sulfonamide antibiotic (sulfamethazine) exiting the capillary. (b). Plot (c) is (b) with background (a) subtracted. SWV conditions: DC voltage ramp : -600 to -1100 in $100 \mathrm{~ms}$; Square-wave frequency/amplitude: $1000 \mathrm{~Hz} / 100 \mathrm{mV} ; 0 \%$ of initial square-wave current response rejected.

Initially, the potential region that was found to illicit the response for the aminoglycoside antibiotics was used for the analysis of these sulfonamides. However, in 
addition to the SWV background disruptions in the 400 to $-800 \mathrm{mV}$ region, a positive SWV response was observed in the extreme negative region of theses scans that was not observed for the aminoglycosides. To better focus on this response, a more negative and narrower potential region was scanned $(-600$ to $-1000 \mathrm{mV})$. Although the $\mathrm{pH}$ of the electrolyte used for the separation of the sulfonamide antibiotics was higher $(\mathrm{pH} 7)$ than that used for dihydrostreptomycin (pH 3) and the background SWV response for these electrolytes was different, it will be shown in work discussed later in this chapter, that the $\mathrm{pH}$ of the electrolyte was not critical to illicit this positive SWV response. A peak presumably due to hydrogen adsorption/reduction was observed at $\sim 650 \mathrm{mV}$ for the background electrolyte (100 mM, pH 7.0 phosphate). The background subtracted response (Figure 3.5c) indicates that a peak-shaped positive response resulted from interaction of the sulfonamide antibiotics with the Pt electrode, which was contrary to the results for dihydrostreptomycin. In other studies, a positive SWV response was not observed with a Au electrode. A similar but lower intensity response was observed with a Pd electrode. Consequently most of the work was carried out with Pt electrodes.

Figure 3.6 shows a 3-dimensional plot of the sulfonamide separation using this adsorption-based detection system (AdsECD). A careful examination of these plots showed that the voltammograms from each of the sulfonamide antibiotics (including trimethoprim) were qualitatively identical, differing only in the magnitude of the response. This was surprising in that one would expect that for analytes with rather varied chemical structure (e.g., the differences between the sulfonamides and trimethoprim), different voltammograms would be observed. This suggests a similarity in the mode of reaction. 
When this response was quantitated using the algorithms described in section 2.3 .4 , the 2dimensional electropherogram shown in Figure 3.7 was obtained.

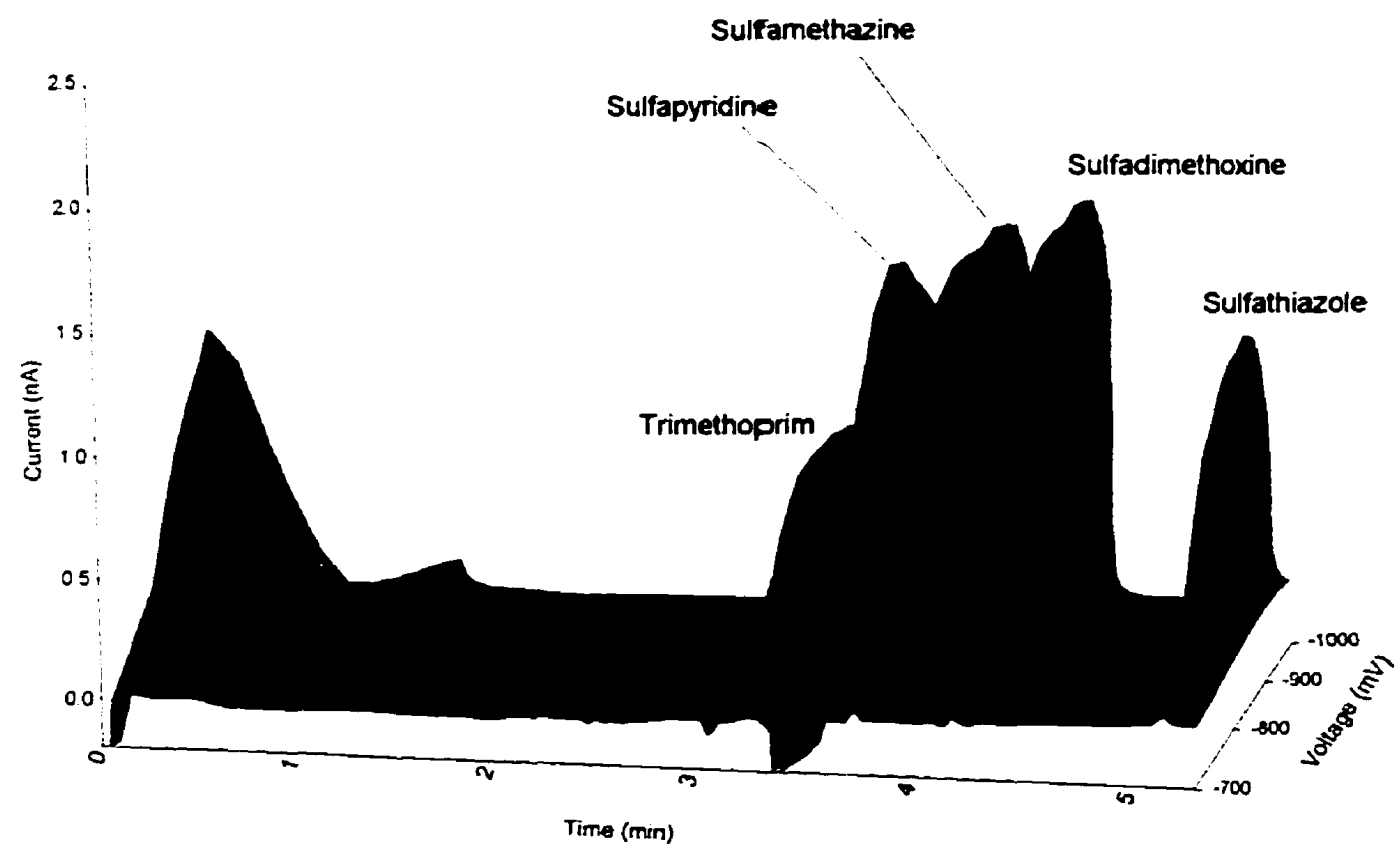

Figure 3.6 -3-D electropherogram of sulfonamide separation. $10 \mu \mathrm{M}$ sulfonamide mixture injected, $2 \mathrm{~s} @$ $5 \mathrm{kV} .10 \mathrm{kV}$ separation voltage. Other separation and SWV conditions described in Figure 3.5

The detection sensitivity obtained using this CE-AdsECD method was compared to that obtained using CE-UV, which is shown in Figure 3.8. The injected concentration in the CE-AdsECD was $1 \mu \mathrm{M}$, but $10 \mu \mathrm{M}$ in the CE-UV separation. A comparison of these electropherograms reveals that a sensitivity improvement of an order-of-magnitude or greater can be obtained using CE-AdsECD over CE-UV detection, even when strongly UV-absorbing analytes are used. Greater advantages are expected for weakly UVabsorbing analytes. By injection of successively lower sulfonamide concentrations, a detection limit of $5 \mathrm{nM}(\mathrm{S} / \mathrm{N}=3)$ was determined for $\mathrm{CE}$-AdsECD. 


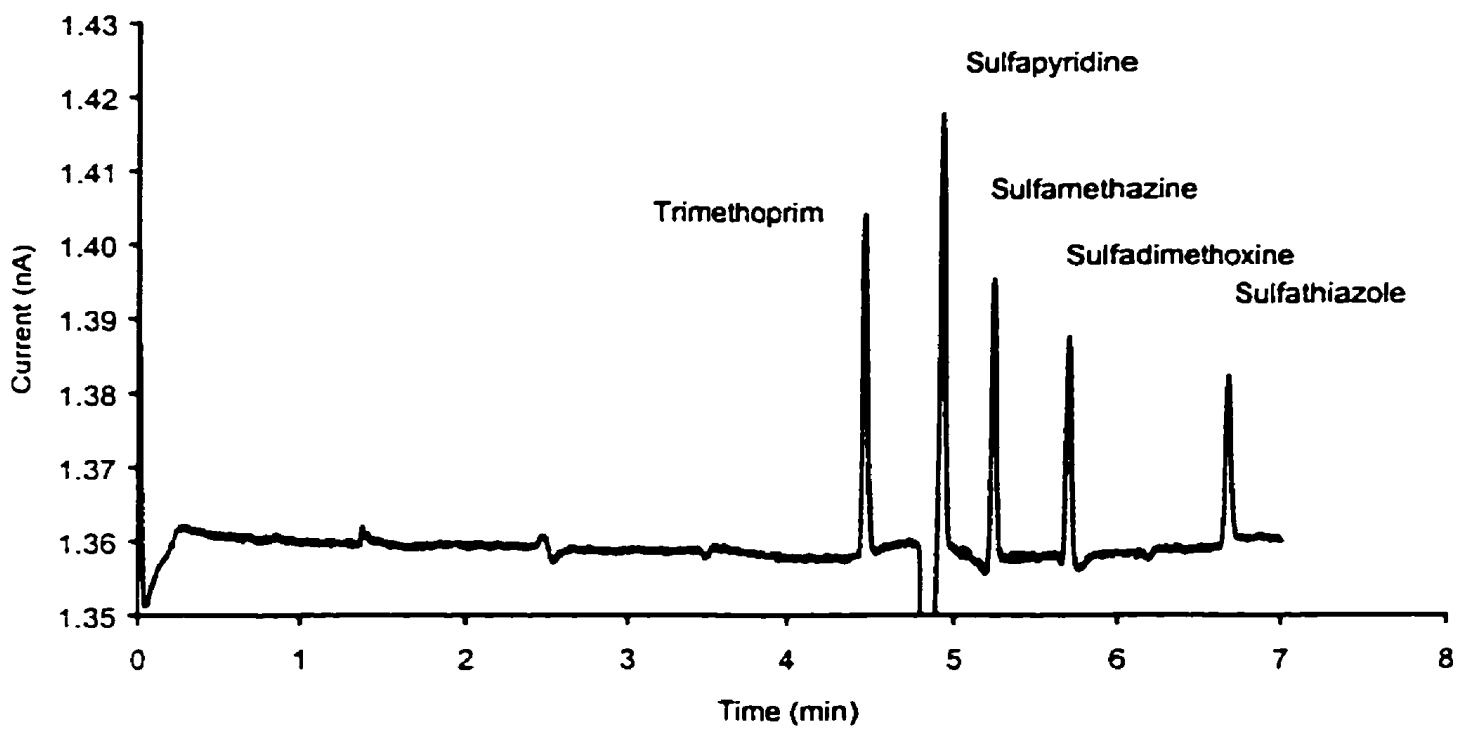

Figure 3.7 - 2-dimensional electropherogram CE-AdsECD separation. $1 \mu \mathrm{M}$ sulfonamide mixture injected. $2 \mathrm{~s} @ 5 \mathrm{kV}$. Separation and SWV conditions described in Figure 3.5

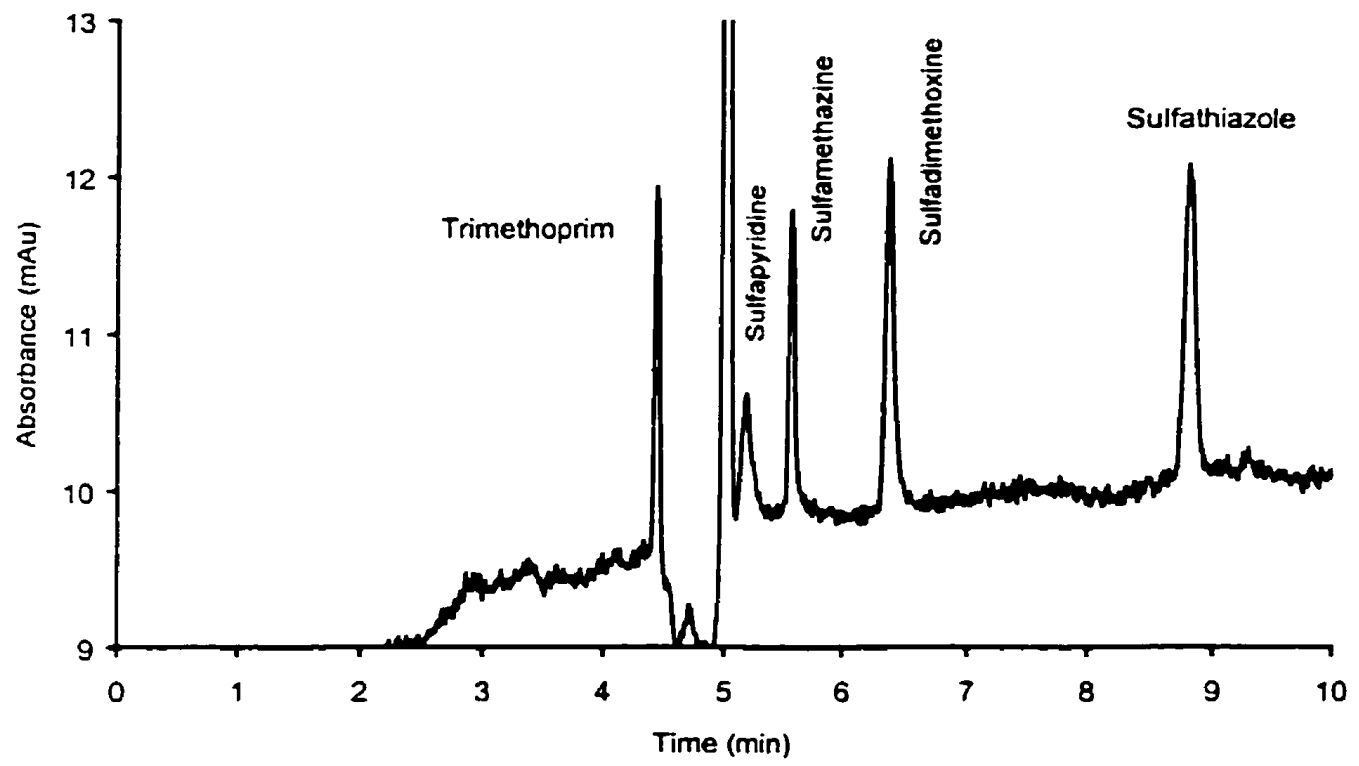

Figure 3.8 - CE-UV separation of the sulfonamide antibiotics. $10 \mu \mathrm{M}$ sulfonamide mixture injected, $2 \mathrm{~s} @$ 0.5 p.s.i. Separation conditions: $15 \mathrm{kV}$ separation potential. $100 \mathrm{mM} \mathrm{pH} 7.0$ phosphate buffer. $\mathrm{UV}$ detection at $195 \mathrm{~nm}$. 


\subsubsection{Optimization of the CE-SWV waveform}

Before this positive SWV response that was observed for the sulfonamide antibiotics is discussed further, it should be noted that voltammetry in these negative potential regions using a Pt electrode has not traditionally been explored. Normally, voltammetry at mm-scale electrodes in these potential regions is severely restricted by both the high currents produced by reaction of the electrolyte with the electrode (hydrogen and oxygen adsorption/reduction) and the resulting generation of gas bubbles at the electrode. While the results in Figure 3.2 and Figure 3.5 clearly show that $\mu \mathrm{m}$-scale electrodes give well defined SWV response in these negative potentials, this advantage of $\mu \mathrm{m}$-scale electrodes has not previously been recognized. For example, in a report discussing the behaviour of $\mu \mathrm{m}$-scale electrodes, Pons and Fleishmann ${ }^{58}$ list the four most common advantages of these smaller scale electrodes as: the very small currents produced can be measured with relative ease; $i \mathrm{R}$ losses are significantly reduced allowing lower conductivity electrolytes to be used; charging currents are reduced; and the rate of mass transfer to and from the electrodes increases with decreasing electrode size. With largerscale electrodes it is difficult to maintain electrode polarization in potential regions where $\mathrm{mA}$ background currents exist. However the $\mu \mathrm{A}$-nA currents of $\mu \mathrm{m}$-scale electrodes can easily be supplied by most potentiostats. Also, because of the increased mass transport, gases produced at the electrode surface have an opportunity to diffuse away before coalescing to form a bubble. In fact, although no analyte response was observed at more negative potentials, scanning to potentials as low as $-1700 \mathrm{mV}$ did not result in unreasonable baseline noise for most electrolytes. It should also be noted, that 
electrochemical detection at potentials below $\sim 0 \mathrm{mV}$ (versus SCE) with conventional electrodes is normally done only after dissolved $\mathrm{O}_{2}$ had been removed from electrolytes by de-aerating solutions to reduce the high background currents the adsorption/reduction of oxygen produces $^{12}$. In the experiments reported here, all electrolytes were used without de-aeration.

Having observed this positive response for the sulfonamide antibiotics, the SWV waveform parameters were investigated to maximize the $S / N$ of the analytical signal. The average $\mathrm{S} / \mathrm{N}$ of the sulfonamides was used to quantify the changes made to the $\mathrm{SWV}$ waveform. The SWV parameters that could be modified were the DC sweep-time, square-wave frequency and amplitude, and the percentage of the initial current response rejected.

The time to complete each SWV scan should be minimized to ensure that adequate temporal resolution can be maintained to accommodate the narrow peak widths. When scan times from 20-150 ms were investigated, $\mathrm{S} / \mathrm{N}$ increased with increasing scan time until $80 \mathrm{~ms}$. DC ramp times above $80 \mathrm{~ms}$ did not result in higher $\mathrm{S} / \mathrm{N}$.

The effect of square-wave frequencies from $400-2000 \mathrm{~Hz}$ was examined. As shown in Figure 3.9, a peak-shaped relationship between square-wave frequency and the sulfonamide $\mathrm{S} / \mathrm{N}$ was observed with the maximum $\mathrm{S} / \mathrm{N}$ occurring at $900 \mathrm{~Hz}$. S/N should increase with increasing square-wave frequency until the capacitance of the data acquisition/electrode/electrolyte system begins to interfere with the measurement of the analyte response. The capacitance of the entire system will depend upon detection 
instrumentation, electrode, electrolyte ionic strength and conductivity, and the analyte diffusion rate. Although $900 \mathrm{~Hz}$ was optimal for this system, each electrolyte/analyte system may have to be optimized independently.

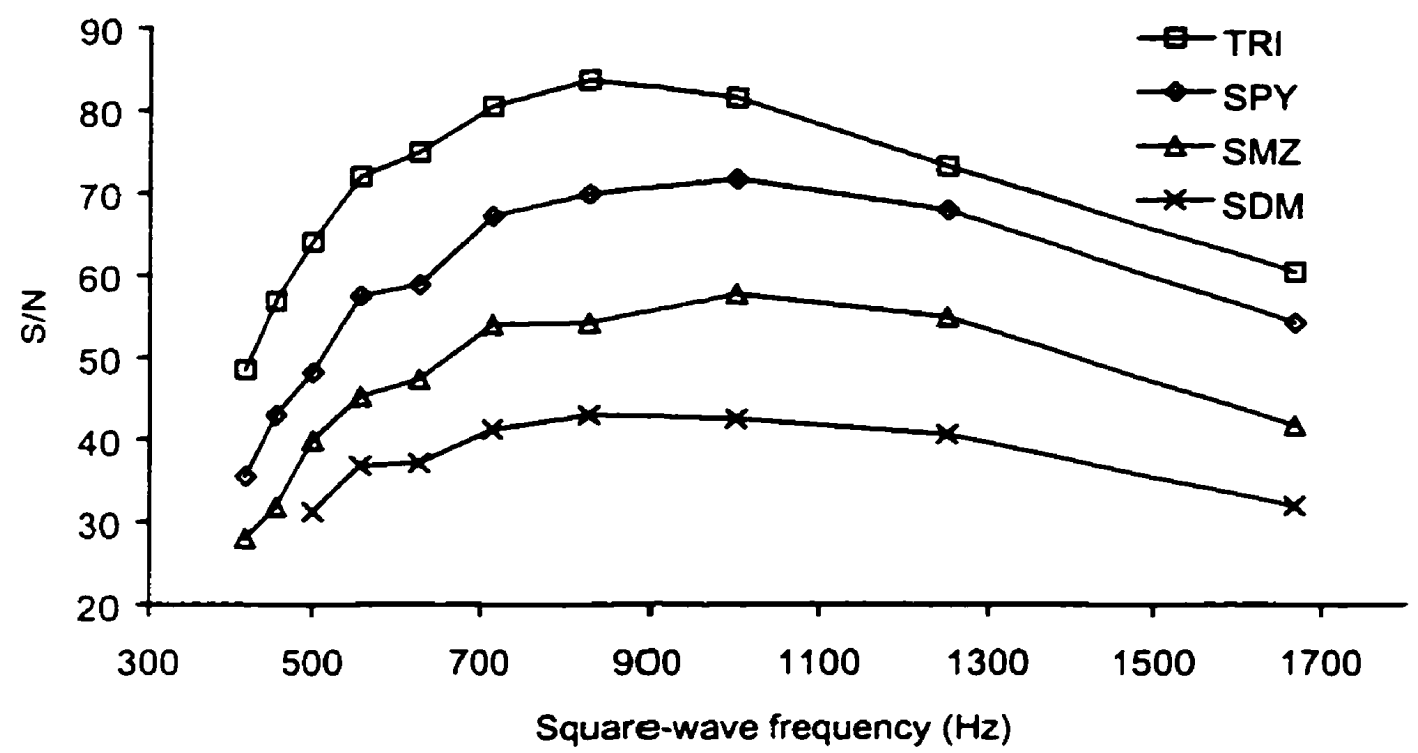

Figure $3.9-$ Effect of square-wave frequency on sulfonamide $S / N$. Based upon $1 \mu \mathrm{M}$ sulfonamide mixture injected, $2 \mathrm{~s} @ 5 \mathrm{kV}$. Separation and SWV conditions described in Figure 3.5.

Similarly, the effect of the square-wave amplitude on $\mathrm{S} / \mathrm{N}$ was plotted for amplitudes from $30-150 \mathrm{mV}$ (Figure 3.10$) . \mathrm{S} / \mathrm{N}$ steadily increased with increasing amplitude reaching a plateau at $130 \mathrm{mV}$. For reversible faradic processes, increasing the square-tvave amplitude above $50 / n$ should result in a broadening of the square-wave voltammogram peak without increasing its height. This trend was not observed for the square-wave voltammograms of the sul fonamides. While some broadening of the voltammogram peak occurred, its peak height increased with increasing square-wave amplitude until $130 \mathrm{mV}$. Because it was difficult to keep the current response within the 
dynamic range of the current amplifiers and digital-to-analog systems when square-wave amplitudes above $\sim 110 \mathrm{mV}$ were used, $100 \mathrm{mV}$ square-wave amplitudes were found to be optimal.

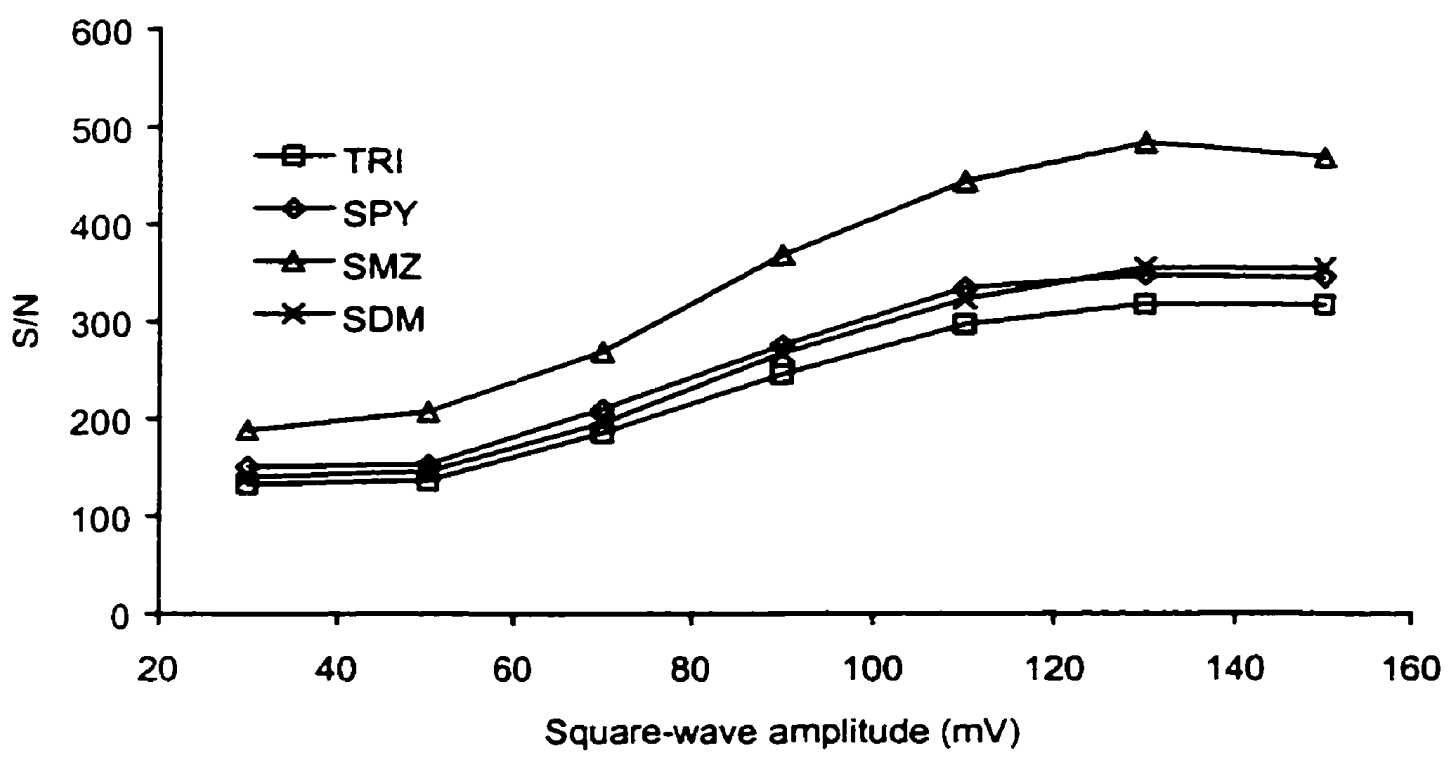

Figure $3.10-$ Effect of square-wave amplitude on sulfonamide $\mathrm{S} / \mathrm{N}$. Based upon $1 \mu \mathrm{M}$ sulfonamide mixture injected, $2 \mathrm{~s} @ 5 \mathrm{kV}$. Separation and SWV conditions described in Figure 3.5.

As was described for SWV detection in Section 2.3.3, the effect of charging current on the measurement was minimized by selection of different portions of the forward and reverse current responses to calculate the differential current. When this method was used to detect the electrochemically active neurotransmitters, the maximum $\mathrm{S} / \mathrm{N}$ was observed when $30 \%$ of the initial forward and reverse currents was rejected. However, for adsorption-based detection of sulfonamides, it was found that $\mathrm{S} / \mathrm{N}$ was greatest when the entire current response was used to calculate the difference current. 
Thus, it appears that the processes that produce the observed current response have frequency dependencies that are higher than purely faradic responses.

The increase in $\mathbf{S} / \mathrm{N}$ with decreasing initial current rejection (Figure 3.11) is due to changes in both the signal and noise. As more data points were used to calculate each point in the square-wave voltammogram, a reduction of noise through signal averaging was obtained. The noise plot in Figure 3.11 shows that noise reduction through signal averaging is realized only when $>40 \%$ of the initial current is rejected. Including more signal data (rejecting less initial current response) did not result in further noise reduction. Another factor effecting $\mathrm{S} / \mathrm{N}$ is the change in the signal. Figure 3.11 shows that the signal decreases almost linearly as more of the initial current response is rejected.

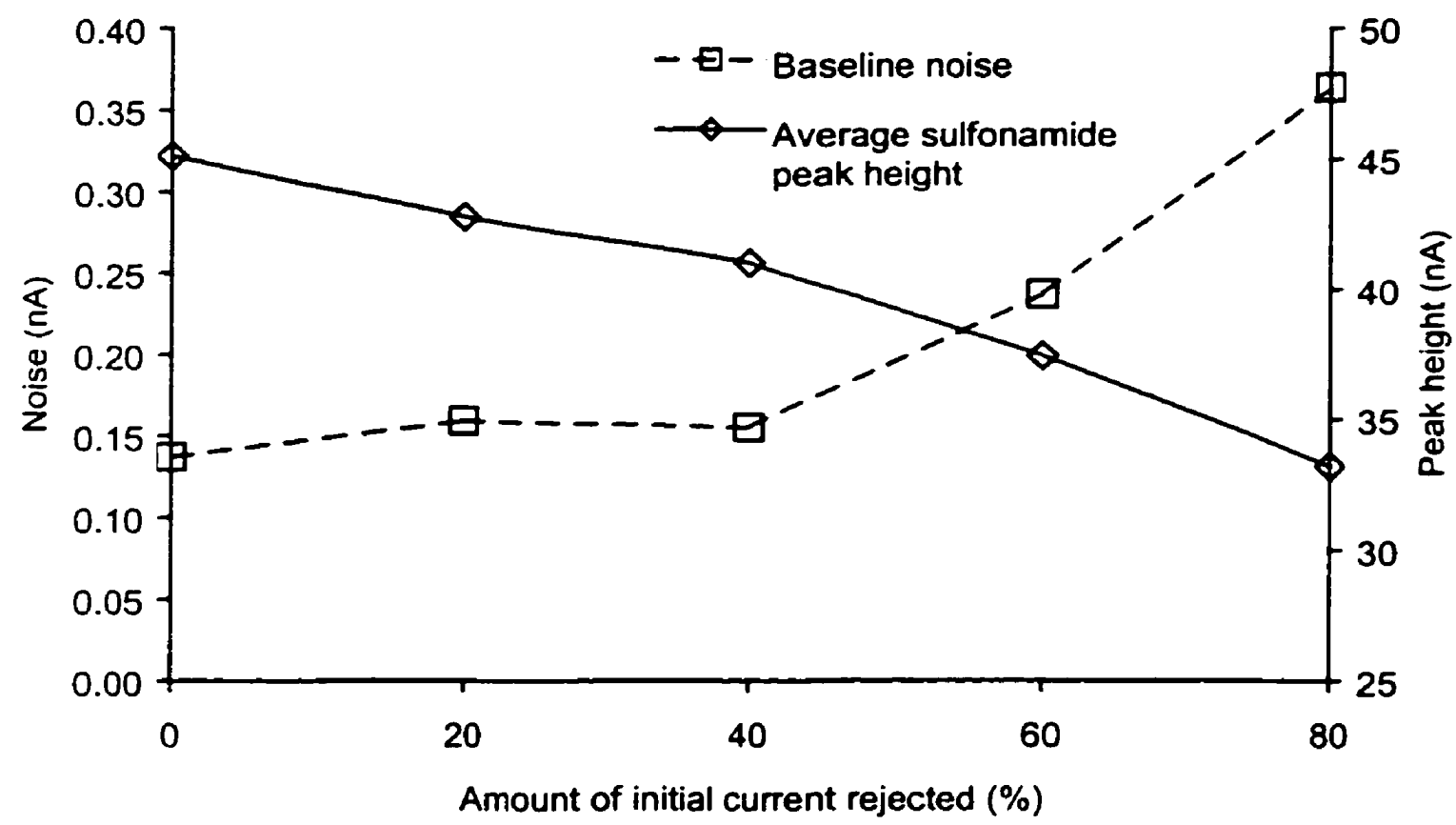

Figure 3.11 - Effect of varied amount of initial square-wave response rejected on sulfonamide peak height and baseline noise. Based upon $1 \mu \mathrm{M}$ sulfonamide mixture injected, $2 \mathrm{~s} @ 5 \mathrm{kV}$. Separation and SWV conditions described in Figure 3.5. 
This was contrary to what was observed when SWV was used to detect electroactive species where a maximum in signal was observed when $30 \%$ of the initial current response was rejected (see Figure 2.6). This indicated that the frequency response of the processes induced by the SWV waveform is higher than the frequency of a response that would be expected from a diffusion-controlled faradic process. Adsorption of the analyte prior to the SWV scan would produce such a response as the process would no longer be diffusion-controlled.

\subsubsection{Post-and pre-SWV scan potential pulses}

Post- and pre-SWV scan potential pulses were initially included in the SWV waveform for the same reasons they were included for the detection of the electroactive neurotransmitters (Chapter 2). The high-voltage post-scan pulse was intended to oxidize the Pt electrode surface to remove any adsorbed analyte or accumulated fouling while the pre-scan was thought to reconstruct the Pt-metal electrode surface and provide a preadsorption step that could be used to concentrate analyte prior to the analytical SWV scan. When the potential and duration of the post- and pre-scan pulses was investigated, rather unexpected and interesting observations were made. Post-scan pulses with potentials from 200-2000 $\mathrm{mV}$ had a significant impact on both the height and shape of the trimethoprim electropherogram peaks. Figure 3.12 illustrates this effect. When a post-scan potential of $2000 \mathrm{mV}$ was used, good peak height was observed with no peak asymmetry. As the post scan potential was lowered, peak heights began to decrease and peak tailing appeared. With post-scan pulses less than $\sim 300 \mathrm{mV}$, the response disappeared completely. The increase in peak tailing was expected because in the potential window of $\sim 500$ to -1000 
$\mathrm{mV}$, analytes may physically and/or chemically adsorb onto a Pt electrode, but there is no mechanism for their subsequent rapid desorption. Thus, slow desorption of accumulated analyte will produce tailing peaks. What was curious was the decrease in the sulfonamide response with decreasing post-scan pulse potentials. From this, it appeared that a highpotential post-scan puise was required to prodice the response that had been observed.

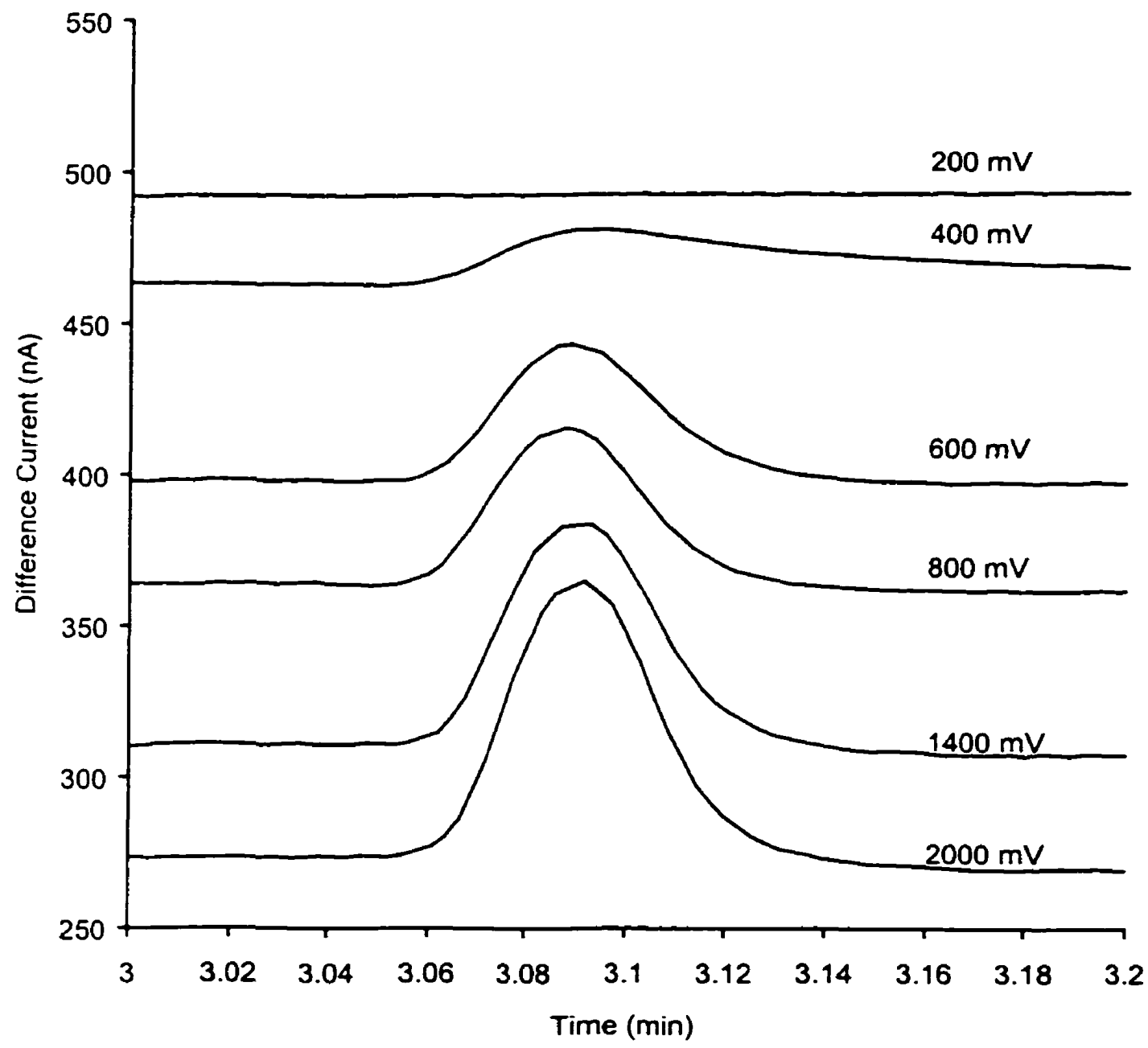

Figure 3.12 - Effect of post-scan pulse potential on trimethoprim electropherogram peak shape. $1 \mu \mathrm{M}$ sulfonamide mixture injected, $2 \mathrm{~s} @ 5 \mathrm{kV}$. Separation and SWV conditions described in Figure 3.5. 
A number of factors could be responsible for the effects observed with highpotential post-scan pulses. One possible explanation is that the $\mathrm{Pt}$ surface was becoming contaminated when low-potential post-scan pulses were used, which caused lower sulfonamide peak heights. Another factor may be the formation of active Pt-metal surfaces created by the oxidation of the Pt surface (which occurs with higher potential post-scan pulses) and subsequent regeneration during the pre-scan pulse. Such surfaces may be extremely active and thus would attract adsorbates strongly. Without this repeated reconstruction of the Pt-metal surface, the activity of the electrode towards adsorbing species may be significantly reduced. Alternatively, the response observed may not be a result of a simple adsorption/desorption process, but may arise from the reduction/adsorption of decomposition products created by the active Pt-oxide surface formed during the high potential pulse. Such an active surface could mediate oxidation of the sulfonamides. This process is supported by the observation that, despite the structural differences between the sulfonamides and trimethoprim, their SWV responses are qualitatively very similar. Other analytes were also found to exhibit similar positive SWV responses. It is possible that the oxidation of these analytes during the high-potential postscan pulse produces similar oxidized species for all analytes, and these products are then adsorbed/reduced during the SWV scan. The mechanism for this positive response may be related to the electrocatalytic oxidation of adsorbed analytes that occurs using pulsed amperometric detection with noble-metal electrodes. This reaction scheme was used by Johnson and LaCourse to introduce pulsed adsorption detection (PAD) of carbohydrates for HPLC in $1990^{59}$. This scheme used electrocatalysis to incite reactions at $\mathrm{Pt}$ and $\mathrm{Au}$ 
electrodes for what were previously thought to be electrochemically inactive analytes. By applying a combination of voltage pulses to the noble metal electrodes, the metal-oxides produced mediated oxidation of various aliphatic organic analytes (aldehydes, simple alcohols, glycols, poly alcohols, carbohydrates) under alkaline conditions to allow anodic amperometric detection (oxidation) at relatively moderate oxidation potentials. The cause(s) of the SWV response is unknown and would likely require extensive study to determine the exact mechanisms. However, at the potentials that were used to detect the sulfonamide antibiotics, the reduction of these surface-catalyzed analytes may be occurring.

The impact of the duration of the post-scan pulse was also investigated. Although lengthening the post-scan pulse would ensure that complete oxidation of the electrode (and/or analyte) was achieved, it will also further lengthen the total applied waveform period, which, ultimately determines the overall data acquisition rate of the detection process. By monitoring the current produced during the post-scan pulse, it was determined that after $\sim 30 \mathrm{~ms}$, the current (due to the oxidation of the Pt surface) had decayed to a steady-state, indicating that complete oxidation of the electrode surface had occurred. Also, when the impact of post-scan pulse times on sulfonamide $\mathrm{S} / \mathrm{N}$ was examined, $\mathrm{S} / \mathrm{N}$ increased with increasing pulse times until $30 \mathrm{~ms}$, but pulse times $>30 \mathrm{~ms}$ did not provide substantial improvement in $\mathrm{S} / \mathrm{N}$. Consequently, $30 \mathrm{~ms}$ was selected as an optimum value for further investigations. 
The pre-scan pulse was thought to have two purposes: re-establishment of the Ptmetal surface and pre-concentration of the analyte prior to the analytical SWV scan. If the pre-scan potential was not set equal to the starting potential of the SWV scan, then the initial portion of the SWV response was distorted due to the potential shift involved in abruptly changing the potential of the electrode from the pre-scan pulse to the initial SWV scan potential. Initially, a second pre-scan pulse was added to re-equilibrate the electrode to the initial SWV scan potential before the scan was applied. However, it was later found to be unnecessary as using pre-scan potentials different than the initial SWV scan potential did not produce significantly different results. Because SWV scans from -600 to -1100 $\mathrm{mV}$ were used, pre-scan potentials of -600 were typically used. The effect of pre-scan pulse time was also investigated. As with the post-scan pulses, the current response observed during the pre-scan pulse was found to decay to a steady-state current after $\sim 20$ $30 \mathrm{~ms}$, which indicated that oxide-free Pt-metal surface had been re-established. To determine if longer pre-scan times would provide pre-concentration of the analyte on the electrode surface to increase the signal produced, longer pre-scan times were also used. Figure 3.13 shows the results of an experiment to examine the effect that pre-scan pulse duration had on sulfonamide $\mathrm{S} / \mathrm{N}$. As can be seen, when pulse times shorter than $30 \mathrm{~ms}$ were used, incomplete reconstruction of the Pt-metal surface appears to decrease $S / N$ (analyte signal decreased slightly and noise was slightly higher). When pulse times longer than 40 ms were used no improvement in analyte signal was observed and noise remained constant. 


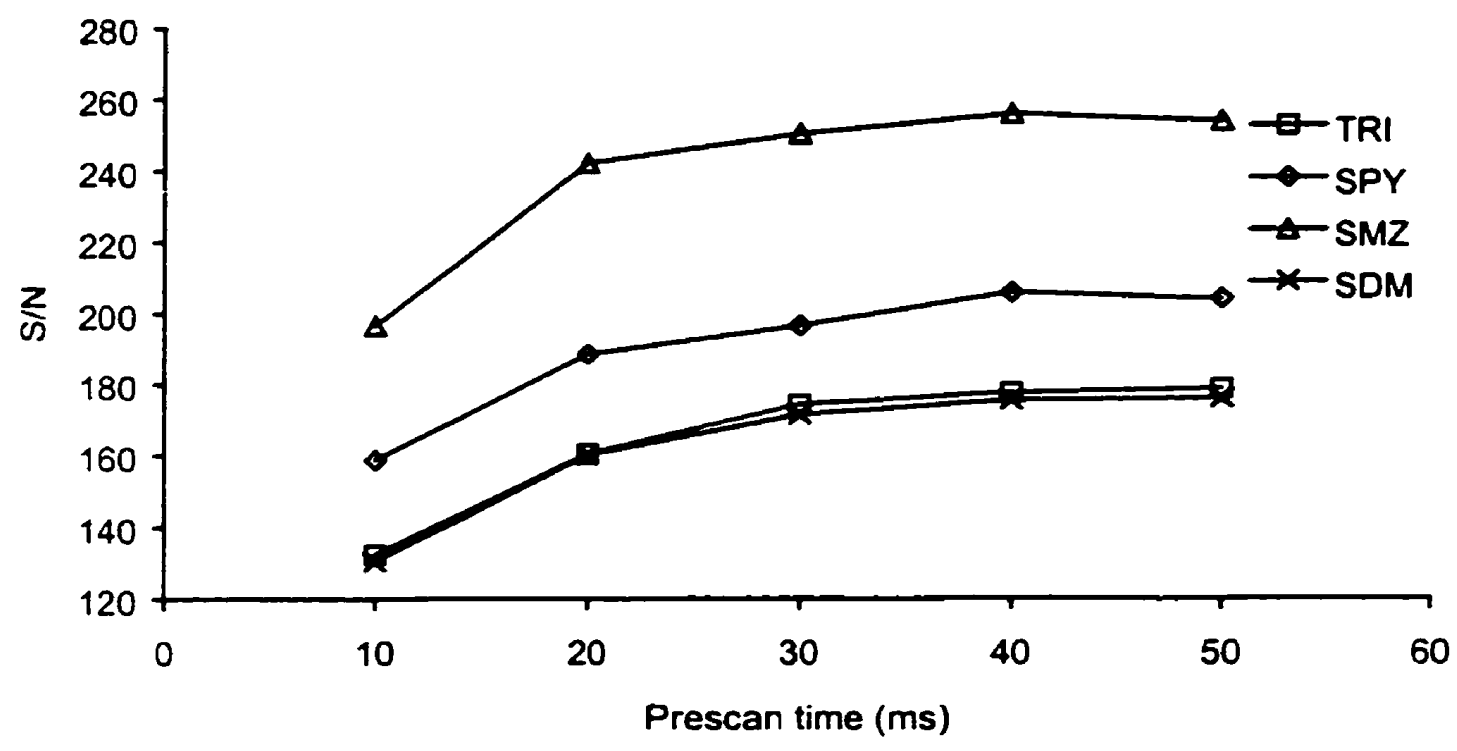

Figure 3.13 - Effect of pre-scan pulse duration on sulfonamide $S / N$. Based upon $1 \mu \mathrm{M}$ sulfonamide mixture injected, $2 \mathrm{~s} @ 5 \mathrm{kV}$. Separation and SWV conditions described in Figure 3.5.

Near the end of this $\mathrm{Ph}$. D. program, it was realized that the current follower/amplifier (Figure 1.4), might not function properly during the post- and pre-scan pulses when rather high currents are generated as the electrode is first oxidized and then reduced to establish a clean-metal surface. This design may cause errors in the values of the applied potentials at the electrode. Since the gain resistor used in Figure 1.4 would be set to amplify typical currents observed during the SWV scan, the high currents during the post- and pre-scan pulses would cause saturation of the op-amp. Thus the potential of the working electrode would drift away from the virtual ground potential held during normal, unsaturated op-amp conditions. As a result, for a desired post-scan pulse of $2000 \mathrm{mV}$, the actual potential of the working electrode was likely drifting from this potential until the current generated during the electrode oxidation was low enough to prevent saturation of the potentiostat op-amp. To alleviate this situation, the microcontroller programming was 
changed to lower the gain of the current follower during pre- and post-scan pulses (a schematic of the programmable gain current follower used in the microcontroller data acquisition system is shown in Figure A.4). After this change was made, the optimum settings for pre- and post-scan pulse potentials and durations were re-examined. Figure 3.14 and Figure 3.15 show that the effect of post-scan pulse potential on the height and efficiency of a trimethoprim peak was significantly different from that reported in Figure 3.12 .

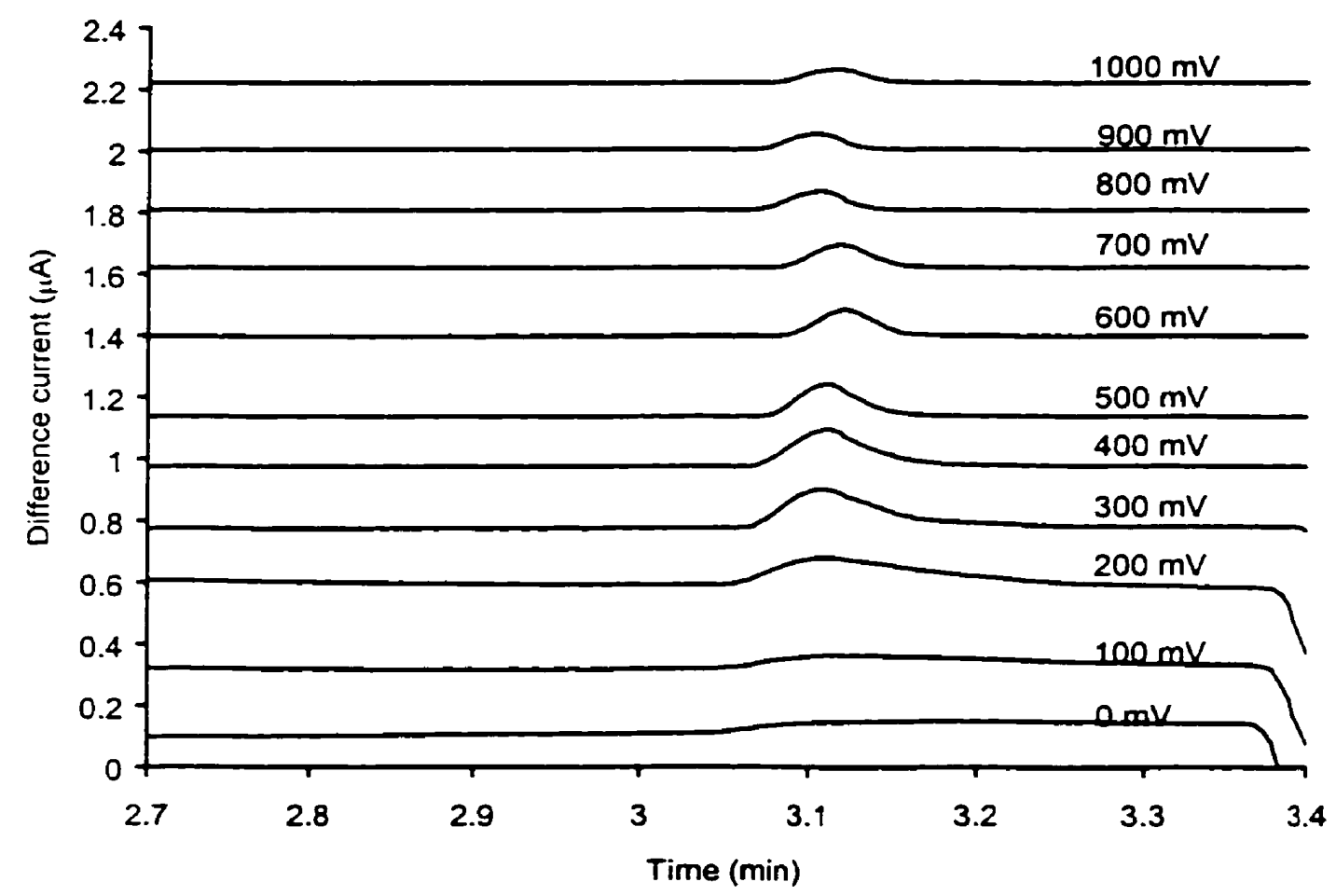

Figure 3.14 - Effect of post-scan potential on trimethoprim peak using new potentiostat configuration in which the current follower gain is lowered during the post- and pre-scan pulses. Based upon $I \mu \mathrm{M}$ sulfonamide mixture injected, $2 \mathrm{~s} @ 5 \mathrm{kV}$. Separation and SWV conditions described in Figure 3.5. 
In Figure 3.12, pulses as high as $2000 \mathrm{mV}$ were used to obtain maximum response, but Figure 3.14 shows that maximum response was now observed at $\sim 400 \mathrm{mV}$. Unfortunately, peak tailing was observed with post-scan pulses of $400 \mathrm{mV}$, indicating that analyte was being slowly removed from the electrode. Only with post-scan potentials above $900 \mathrm{mV}$ did peak tailing disappear.

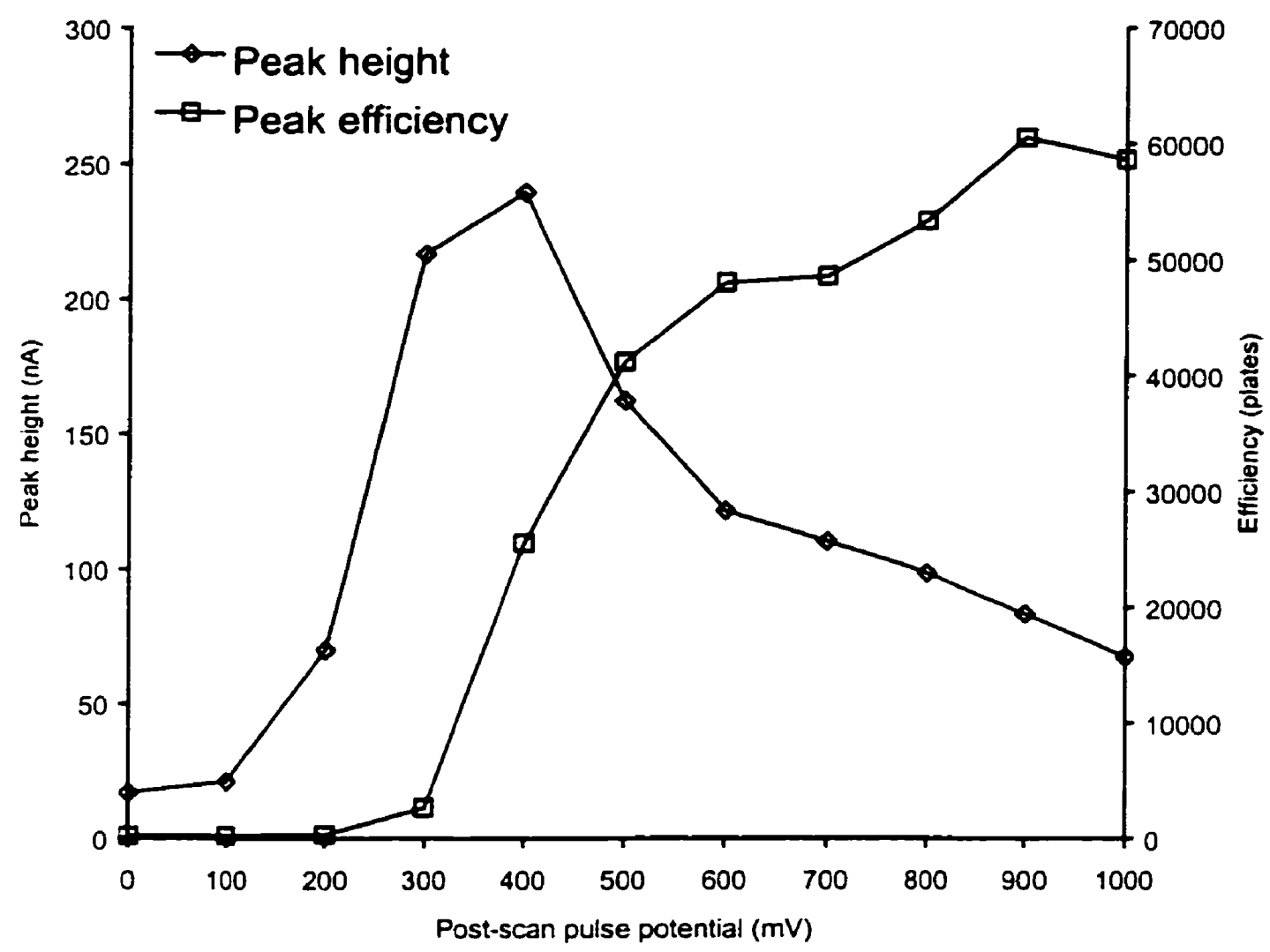

Figure 3.15-Effect of post-scan pulse potential on trimethoprim $\mathrm{S} / \mathrm{N}$ and peak efficiency using new potentiostat configuration in which the current follower gain is lowered during the post- and pre-scan pulses. Based upon I $\mu \mathrm{M}$ sulfonamide mixture injected. $2 \mathrm{~s} @ 5 \mathrm{kV}$. Separation and SWV conditions described in Figure 3.5.

The formation of a surface oxide has been shown to play an important role in the electrocatalysis of aliphatic compounds and their subsequent oxidation at noble metal 
surfaces (pulsed amperometric detection, LaCourse and Dasenbrock ${ }^{12}$ ). The development of oxides at Pt electrodes is thought to start by the formation of adsorbed hydroxyl radicals (Pt-OH) starting at $\sim 150 \mathrm{mV}^{12}$. More positive potentials $(\sim>700 \mathrm{mV})$ will result in further oxidation of the $\mathrm{Pt}$ surface to $\mathrm{Pt}-\mathrm{O} ; \mathrm{Pt}-\mathrm{O}_{2}$ formation can occur with high overpotentials and longer times ${ }^{60}$. From the post-scan potential study shown in Figure 3.15, it appears that this initial Pt-OH oxide may also be important in AdsECD. Also, effective desorption of the analyte from the electrode surface appears to coincide with formation of $\mathrm{Pt}-\mathrm{O}$ oxide. This behaviour has also been observed in pulsed amperometric detection $^{12}$.

In addition to the changes found for optimum post-scan pulse potential, it was also discovered that much shorter post- and pre-scan pulses could be used. This is again due to the fact that when the gain of the current follower was high during these extreme potential excursions, high working electrode currents resulted in drifting of the working electrode potential. Post-scan pulses of $30 \mathrm{~ms}$ were previously required for optimum results, but the gain-lowered potentiostat was effective with only $5 \mathrm{~ms}$ pulses (with the lower potentials discussed above).

As shown in Figure 3.15, although a $400 \mathrm{mV}$ post scan pulse results in the maximum response, post-scan pulses $>900 \mathrm{mV}$ are required to effectively desorb analyte from the electrode and eliminate peak tailing. Preliminary studies have shown that by using a $5 \mathrm{~ms}, 1000 \mathrm{mV}$ post-scan pulse (to desorb analyte) and adding an additional prescan pulse of $400 \mathrm{mV}$ for $5 \mathrm{~ms}$ (possibly to prepare electrocatalytic Pt-OH oxide) to the 
waveform, both good sensitivities and peak shape could be obtained. This modified waveform has also resulted in reduced baseline noise for low-pH electrolytes as well as slightly better analyte responses. Obviously, a more complete investigation of the impact the changes made to the potentiostat system is necessary to confirm the optimum design for the potentiostat circuitry.

It should be noted that these gain-lowering potentiostat circuit modifications were done late in this Ph.D. program. The work reported in the following sections was done using the fixed-gain potentiostat system.

\subsubsection{Effect of analyte structure on SWV response}

Although initial experiments were designed to develop a detection system that responded to physical adsorption of analytes, the response observed for the sulfonamide antibiotics suggested that, a more sensitive response resulted from a more complex chemisorption process. The obvious difference between the sulfonamides and compounds that produced only a physical adsorption-based response is the aromaticity present in the sulfonamides. Whereas the adsorption of aliphatic compounds is dominated by hydrophobic interactions leading to physical adsorption ${ }^{52}$, the "available" $\pi$-resonance stabilized electrons in the aromatic sulfonamide analytes appeared to result in a more complex chemisorption process. To verify the importance of resonance-stabilized electron density in this detection method, several amino acids were investigated. Amino acids were chosen because they are easily separated by CE due to their charged functional groups, but more importantly they are a homologous series that can be used to explore the importance of various functional groups on detector response. The amino acids 
investigated are shown in Figure 3.16.<smiles>CC(N)C(=O)O</smiles><smiles>NC(Cc1ccc(O)c(O)c1)C(=O)O</smiles><smiles>NC(CO)C(=O)O</smiles>

Phenylalanine<smiles>NC(Cc1ccccc1)C(=O)O</smiles>

Figure 3.16 - Structures of the amino acids used to investigate the importance of functionality in detector response.<smiles>CSCCC(N)C(=O)O</smiles>

The background subtracted SWV responses taken from the CE separation of these amino acids are shown in Figure 3.17. What is interesting with the responses for each of these amino acids is that they are qualitatively very similar. Also, the amino acids SWV response is similar to that produced by the sulfonamides. The importance of the presence of resonance-stabilized electron density is illustrated as well in Figure 3.17. 


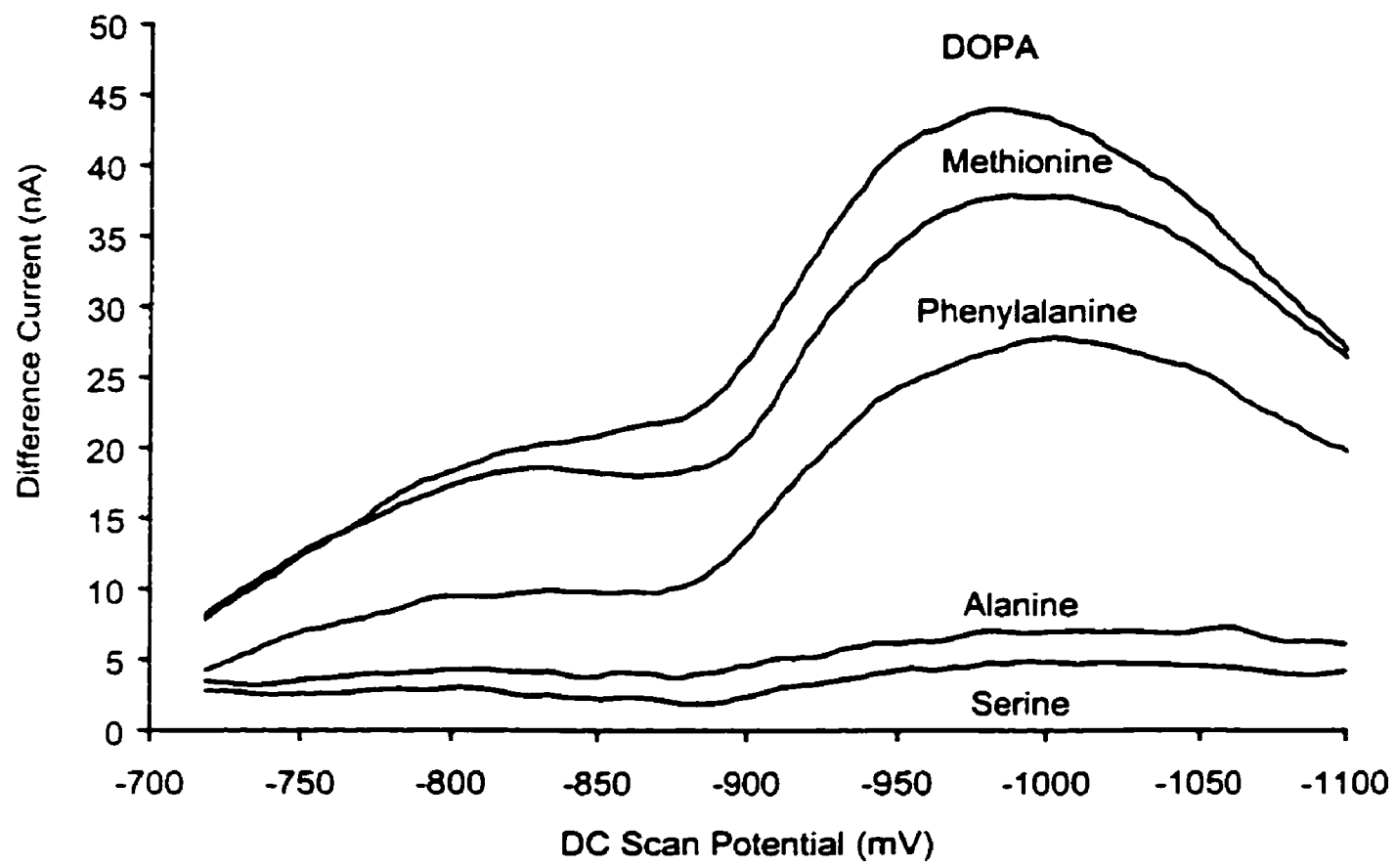

Figure 3.17 - Background subtracted SWV response produced by amino acids. Scans captured during a CE separation as each analyte zone exited the capillary. Electrolyte: $100 \mathrm{mM}, \mathrm{pH} 7.0$ phosphate buffer. SWV conditions: DC voltage ramp : -700 to -1100 in $100 \mathrm{~ms}$; Square-wave frequency/amplitude: 1000 $\mathrm{Hz} / 100 \mathrm{mV} ; 0 \%$ of initial square-wave current response rejected.

Alanine and serine both give lower, but positive responses, presumably due to the resonance-stabilized $\pi$-electron density in the carbonyl group. When a phenyl group is added to alanine (phenylalanine), a response with an order-of-magnitude greater intensity was observed. Similarly, excellent responses were also observed for DOPA and methionine. The high methionine response may be a result of chemisorption via the non. bonding valence electron pairs of sulfur in its -2 oxidation state. Although the sulfonamides also contain a sulfur atom, it is in a +6 oxidation state, and thus has no lonepair valence electrons to promote strong chemisorption. As a result, the magnitude of the trimethoprim (no sulfur) response was essentially the same as that shown for the sulfonamides in Figure 3.5. 


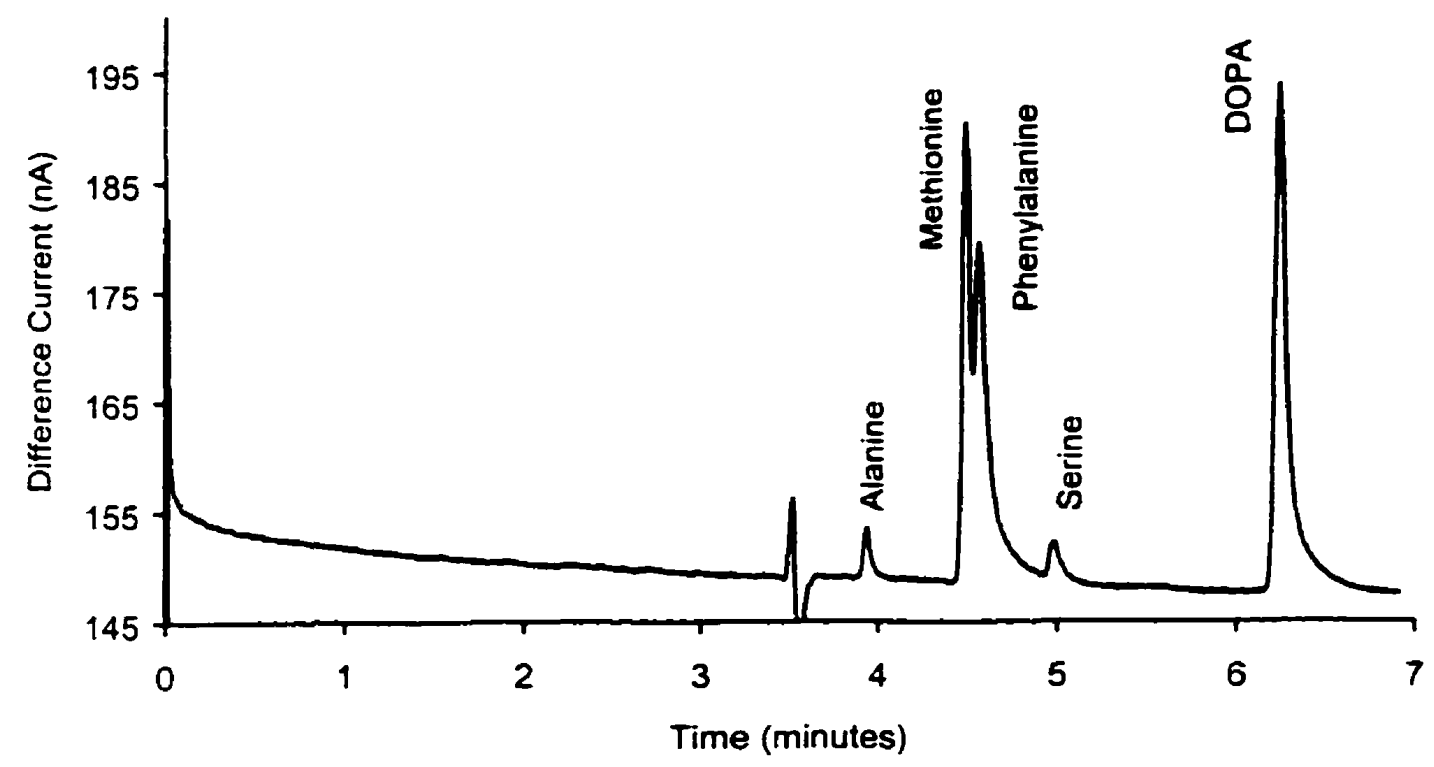

Figure 3.18 - CE-AdsECD amino acid separation. $10 \mathrm{mM}$ alanine, phenylalanine, serine and methionine injected electrokinetically, $5 \mathrm{~s} @ 10 \mathrm{kV}$. Separation voltage: $10 \mathrm{kV}$. Other separation and SWV conditions shown in Figure 3.17.

A separation of these amino acids is shown in Figure 3.18. Although the magnitude of the response for the non-aromatic amino acids alanine and serine is low, the detection limits that can be achieved $(\sim 1 \mu \mathrm{M}, \mathrm{S} / \mathrm{N}=3)$ are far better than those that can be achieved using UV detection as these analytes do not have UV-Vis chromophores. The detection limits for phenylalanine, DOPA and methionine (as well as other amino acids with similar functionality) are similar to those obtained for the sulfonamides.

At this point, it appears that response may be related to a combination of physical, chemisorption and oxidation/reduction effects. The magnitude of the response appears to be related to the strength of the analyte-Pt interaction and thus is stronger for species that have phenyl or sulfur $(-2)$ functional groups that interact strongly with activated $\mathrm{Pt}$ 
surfaces. This conclusion is confirmed by the results for other types of analytes that are reported later in this thesis.

\subsubsection{Application of CE-AdsECD}

To more fully evaluate the analytical features of CE-AdsECD, a variety of analyte groups were separated and detected. These separations were performed using a wide variety of separation buffers, $\mathrm{pH}$, ionic strength, organic modifiers, micellar systems, and chiral selectors to further determine the effect these had on the application of this technique.

\subsubsection{CE-AdsECD separations of basic drugs of forensic interest}

Hudson et al recently reported CE-UV methods for the separation and detection of a wide variety of basic drugs ${ }^{61.62}$ commonly encountered in forensic analysis. Currently, this method is in use in the Royal Canadian Mounted Police (RCMP) forensic laboratories for the routine screening of 20 basic drugs ${ }^{63}$. This same drug mixture was analyzed using both CE-UV (Figure 3.19b) and CE-AdsECD (Figure 3.19a) to determine the relative detection limits that could be obtained. Because this separation is performed using a low $\mathrm{pH}$ electrolyte, it was also an opportunity to observe the effect that a low $\mathrm{pH}$ electrolyte had on the AdsECD response since sulfonamides and amino acids separations were performed using pH 7 and 9 electrolytes, respectively. Both CE-UV and CE-AdsECD separations shown are injections of $1 \mu \mathrm{g} / \mathrm{mL}$ basic drug mixtures. 


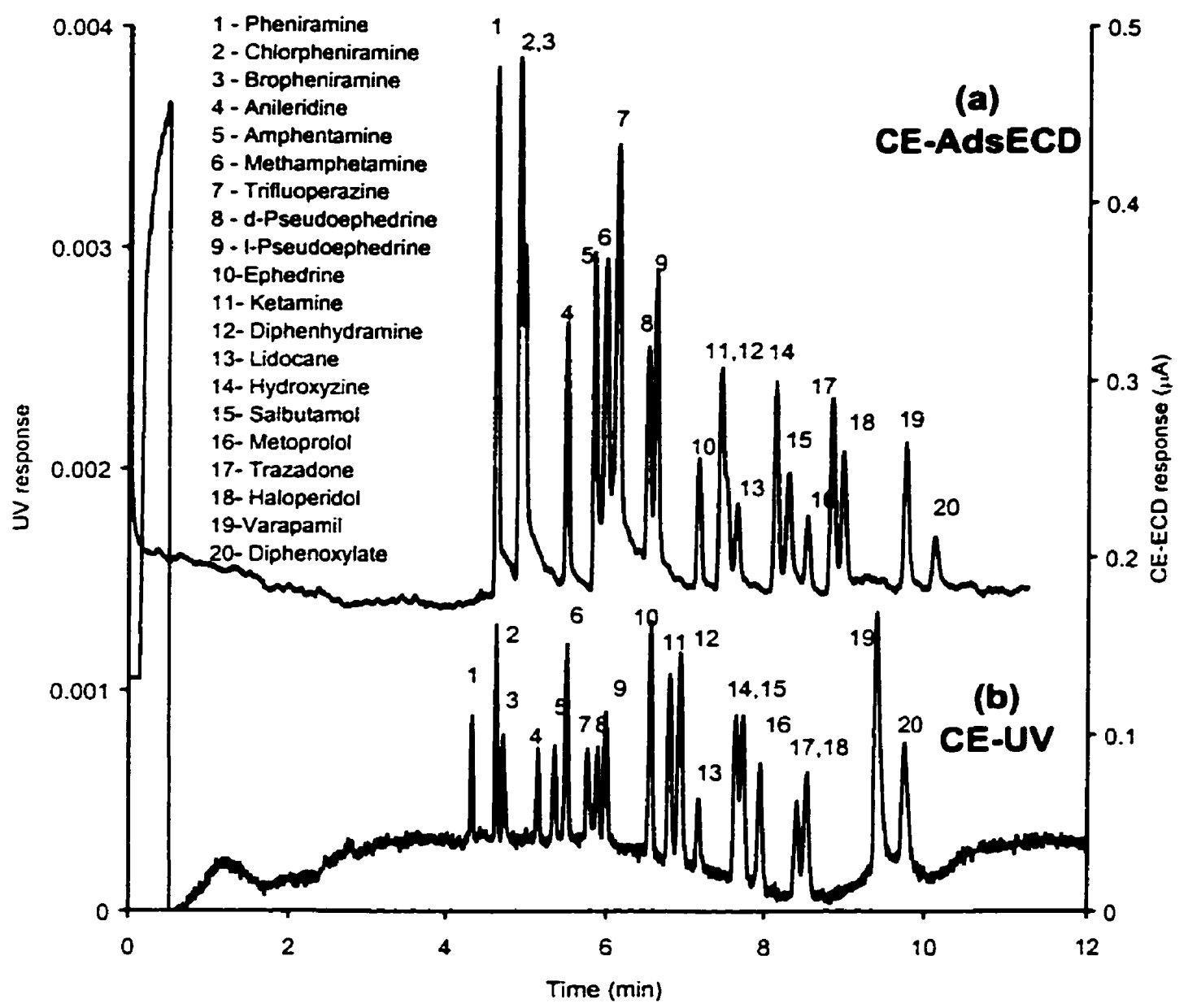

Figure 3.19 - CE-UV at $195 \mathrm{~nm}$ (b) and CE-AdsECD (a) separations of RCMP QC mixture. Separation conditions: $100 \mathrm{mM}$, pH 2.38 phosphate buffer, 1 ppm standard solution RCMP drug QC mixture injected electrokinetically, $1 \mathrm{~s} @ 10$ kV (CE-AdsECD, plot a), pressure injected, $5 \mathrm{~s} @ 0.5$ p.s.i. (CE-UV, plot b). SWV conditions: DC voltage ramp : -600 to -1100 in $100 \mathrm{~ms}$; Square-wave frequency/amplitude: 1000 $\mathrm{Hz}^{\prime} \mathrm{L} 100 \mathrm{mV} ; 0 \%$ of initial square-wave current response rejected.

The better separation observed for the CE-UV plot (Figure 3.19b) is likely due to the higher field strength used $(30 \mathrm{kV} / 47 \mathrm{~cm}$, the CE-AdsECD instrument was capable of only $10 \mathrm{kV} / 30 \mathrm{~cm}$ ); separation efficiency is directly related to the separation field strength. Although the injection parameters used for each system were optimized to maximize the $\mathrm{S} / \mathrm{N}$ for samples injected in the separation buffer, electrokinetic injection was used for the 
CE-AdsECD separation (Figure 3.19a), and thus analytes with longer migration times were injected less; pressure injection was used for the CE-UV separation. Considering this, it appears that higher $\mathrm{S} / \mathrm{N}$ was obtained using CE-AdsECD for these analytes.

Although the structures of these drugs are not shown here, all of the drugs separated contain some degree of aromaticity and, like the sulfonamides and amino acids, the SWV response was qualitatively identical. At this low $\mathrm{pH}(\mathrm{pH} 2.38)$, a low frequency $(-0.1-1 \mathrm{~Hz})$ baseline fluctuation was observed. This was likely due to the generation of microscopic hydrogen bubbles on the electrode surface that diffuse before coalescing. Visible gas bubbles did form occasionally between the capillary-electrode junction which resulted in severe baseline disturbances and required purging of the detection ceil. Even though the magnitude of the analyte signal observed for these basic drugs was comparable to that observed for the sulfonamide antibiotics, this increased baseline noise at low $\mathrm{pH}$ resulted in detection limits about an order of magnitude higher $(50-300 \mathrm{ppb}, \mathrm{S} / \mathrm{N}=3)$.

\subsubsection{CE-AdsECD enantiomeric separations of 2-HMP and Deprenyl}

Deprenyl (Figure 3.20a) is a drug used to treat neurological disorders including Alzheimer's disease. 2-HMP (Figure 3.20b) is a drug that is currently being developed to treat similar disorders while reducing unwanted side effects typically encountered using Deprenyl. Before drugs can be approved for use in most countries, manufacturers must demonstrate the enantiomeric purity of their products. CE with chiral selectors added to the separation electrolyte has become an extremely powerful tool for enantiomeric separations ${ }^{64}$. 
a) Deprenyl<smiles>C#CCN(C)C(C)Cc1ccccc1</smiles>

b) 2-HMP

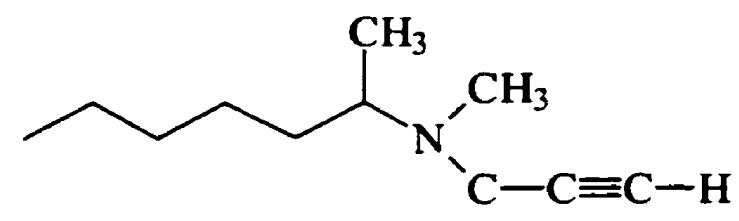

Figure 3.20-Structures of Deprenyl and 2-HMP.

The Department of Neuropsychiatric Research at the University of Saskatchewan (Saskatoon, SK), has been successful in resolving the enantiomers of Deprenyl using a variety of cyclodextrin-based chiral selectors ${ }^{65}$, but has not been able to detect $2-$ HMP using UV detection to enable verification of its enantiomeric purity. Because of the $\pi$ electron density propargyl group (-C-C $\equiv \mathrm{C}-\mathrm{H})$ present in 2-HMP, it was considered a possible candidate for AdsECD detection. The investigation of the AdsECD behaviour for this analysis problem was important in answering two main questions. Firstly, "Can AdsECD be applied to basic drugs that are difficult to detect by other means?," and secondly, "Will the presence of cyclodextrins have detrimental effects?." The latter question is particularly important because of the widespread use of cyclodextrins for the separation of a wide variety of enantiomeric drugs.

Figure 3.21 shows a separation of both Deprenyl and 2-HMP without (a) and with (b) the chiral selector $\beta$-cyclodextrin added to the separation electrolyte. Although the response produced by $2-\mathrm{HMP}$ is of slightly lower magnitude than that produced by Deprenyl. it still allows detection of 2-HMP to $\sim 500 \mathrm{nM}$ levels which will allow verification of its enantiomeric purity. The separations in Figure 3.21 clearly show that the addition of cyclodextrin to the separation electrolyte did not impair AdsECD detection. 


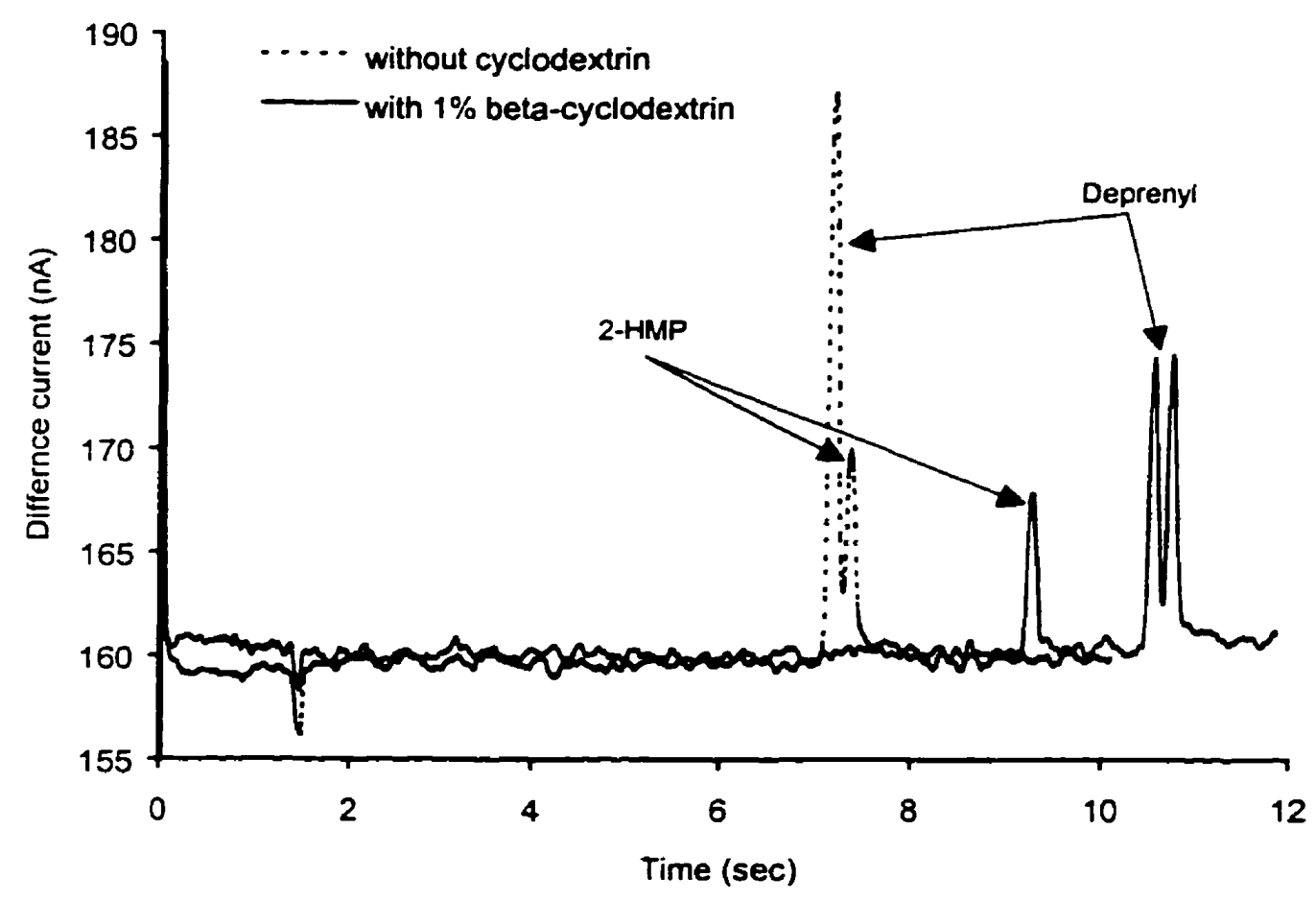

Figure 3.21 - Separation of Deprenyl and 2-HMP with and without $1 \% \beta$-cyclodextrin added. Separation conditions: $20 \mathrm{mM} \mathrm{pH} 3$ phosphate buffer, $10 \mathrm{kV}$ separation voltage, $10 \mu \mathrm{M}$ Depreny/2-HMP standard injected, $3 \mathrm{~s} @ 10 \mathrm{kV}$. SWV conditions: DC voltage ramp : -600 to -1100 in $100 \mathrm{~ms}$; Square-wave frequency/amplitude: $1000 \mathrm{~Hz} / 100 \mathrm{mV} ; 0 \%$ of initial square-wave current response rejected.

As can be seen, while the entantiomers of Deprenyl are successfully separated using $\beta$-cyclodextrin those of 2-HMP are not. A number of other cyclodextrin-based chiral selectors have been attempted and, while no negative impact has been observed on the AdsECD detection method, none have yet resolved the 2-HMP enantiomers. Like the basic drugs of forensic importance discussed in the previous section, these drugs were separated using a low $\mathrm{pH}$ electrolyte (typically $\mathrm{pH} 2-3$ ) and baseline noise increased the overall $\mathrm{S} / \mathrm{N}$ of AdsECD (200/500 nM Deprenyl/2-HMP, $\mathrm{S} / \mathrm{N}=3$ ). It was found during this investigation that using lower ionic strength (i.e., $<50 \mathrm{mM}$ ) separation electrolytes reduced 
this background noise. Consequently, it should be possible to improve the detection limits discussed for the drugs in Section 3.3.5.1.<smiles>CC(C)NCC(O)c1cc(Cl)c(N)c(Cl)c1</smiles><smiles>CCNCC(O)c1cc(O)cc(O)c1</smiles><smiles>CC(C)CNCC(O)c1ccc(O)c(CO)c1</smiles><smiles>CC(C)NCC(O)c1ccccc1Cl</smiles><smiles>Oc1cc(O)cc(C(O)CNCCc2ccccc2)c1</smiles>

Figure 3.22 - Structures of the $\beta$-agonists.

\subsubsection{CE-AdsECD separations of $\beta$-agonists used for illicit veterinary applications}

The $\beta$-agonists are a homologous series of $\mathrm{N}$-alkylphenyl (or pyridyl) ethanol amines (see Figure 3.22). Although some $\beta$-agonists drugs have been approved for use in the treatment of a variety of respiratory illnesses in cattle, these drugs have also been found to be effective in growth promotion (an application that has not received approval), particularly for calves used in veal production. Because of the possibility of drug residues being present in the meat from animals treated in this manner, regulatory agencies have been developing sensitive techniques for detecting these, particularly in the European Union where, although all growth promoters have been banned, wide-spread illegal use is 
suspected. Capillary electrophoresis is particularly attractive for the separation of these drugs as the important $\beta$-agonists can be separated in less than 10 min whereas, significantly longer runtimes are required using HPLC because of the varied hydrophobicity of these analytes ${ }^{66}$. However, as is common in CE, although separation can be obtained, poor detection limits with CE-UV prevent trace-residue application. Because of the presence of the phenyl group in these drugs (Figure 3.22), it was expected that CE-AdsECD would be effective. Figure 3.23 shows that good $C E$ separation of the five $\beta$-agonists is possible, and that sensitivities and peak shapes are good.

As with the sulfonamide antibiotics, $\beta$-agonists all have good UV-chromophores. Despite this, the sensitivities obtainable using CE-AdsECD were $\sim 100 \mathrm{x}$ better than those that were obtained using CE-UV. The electropherogram shown in Figure 3.23 was obtained by injecting the $\beta$-agonists dissolved in the separation electrolyte. When these analytes were dissolved in water (no electrolyte) the results in Figure 3. 24a show that the concentration effects produced by stacking ${ }^{67}$ resulted in improved detection limits ( -500 ppt were obiainable, equivalent to $\sim 1-3 \mathrm{nM}, \mathrm{S} / \mathrm{N}=3$, estimated from electropherograms CE-AdsECD electropherogram shown in Figure 3.24a). Figure 3.24 also shows that with this stacking technique, sensitivities obtained using CE-AdsECD compare well with those available using HPLC-MS-MS ${ }^{66}$, currently one of the most sensitive methods of analysis used for $\beta$-agonists. A HPLC method recently published for the fluorescence detection of $\beta$-agonists in urine reported detection limits of $3 \mathrm{ppb}(\mathrm{S} / \mathrm{N}=3)$ for standards solutions of similar drugs ${ }^{68}$. These comparisons suggest that CE-AdsECD can compete with the tracelevel sensitivities obtained using HPLC methods. For this group of compounds, CE is 
particularly attractive as separations based upon HPLC suffer from having widely different retention times on typically reversed phase columns ${ }^{66}$. Although only five $\beta$ agonists where separated in this report, $\mathrm{CE}$ has been shown to be very effective in separating ten of the most common $\beta$-agonists in under 10 minutes $^{69}$.

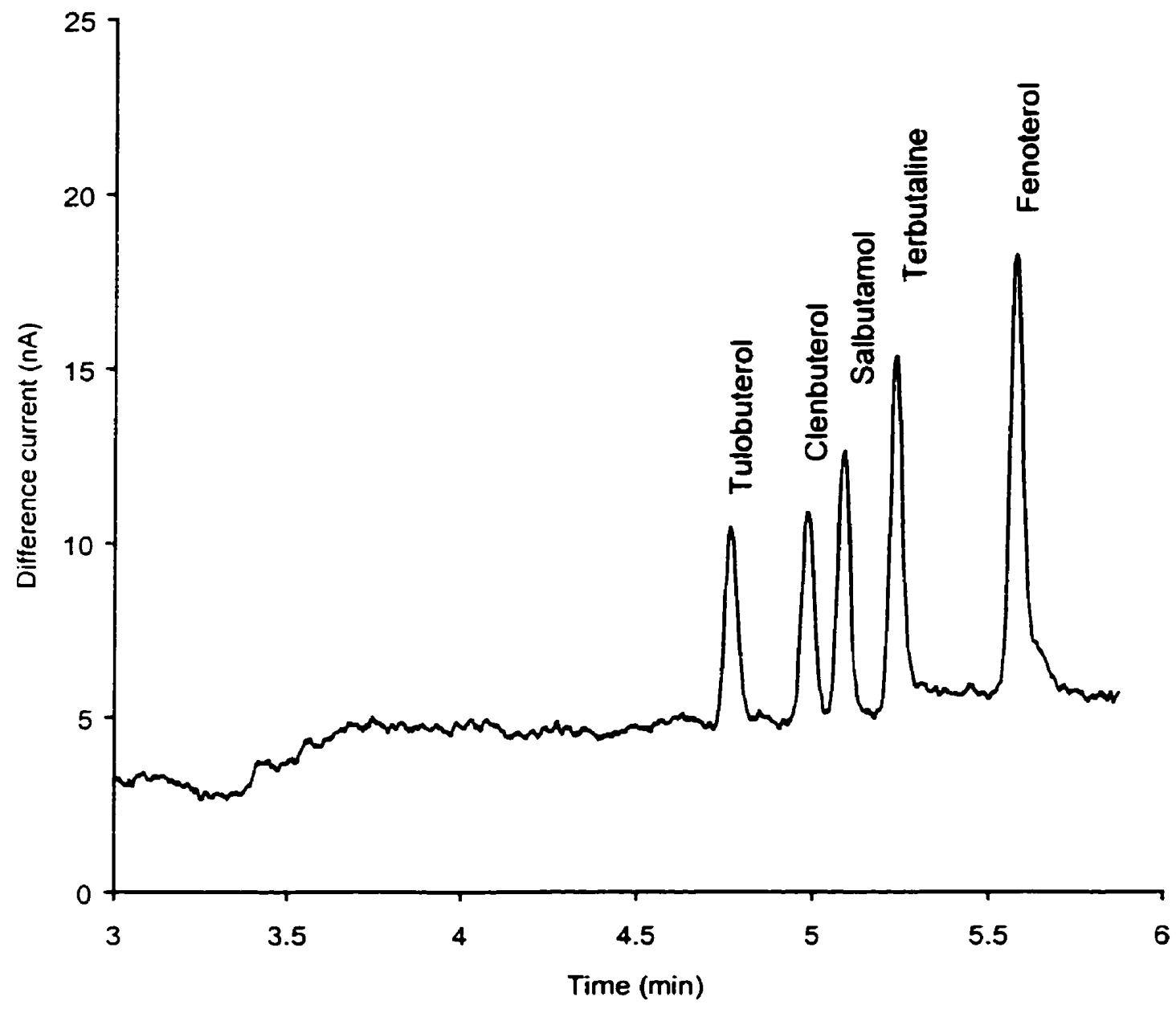

Figure 3.23 - CE-AdsECD separation of $\beta$-agonists. Separation conditions: $100 \mathrm{mM}, \mathrm{pH} 7.0$ phosphate buffer with $15 \%$ methanol added, $10 \mathrm{kV}$ separation voltage. $100 \mathrm{ppb}$ solution of tulobuterol, clenbuterol, salbutamol, terbutaline and fenaterol injected, $4 \mathrm{~s} @ 10 \mathrm{kV}$. SWV conditions: DC voltage ramp : -600 to 1100 in $100 \mathrm{~ms}$; Square-wave frequency/amplitude: $1000 \mathrm{~Hz} / 100 \mathrm{mV}: 0 \%$ of initial square-wave current response rejected. 

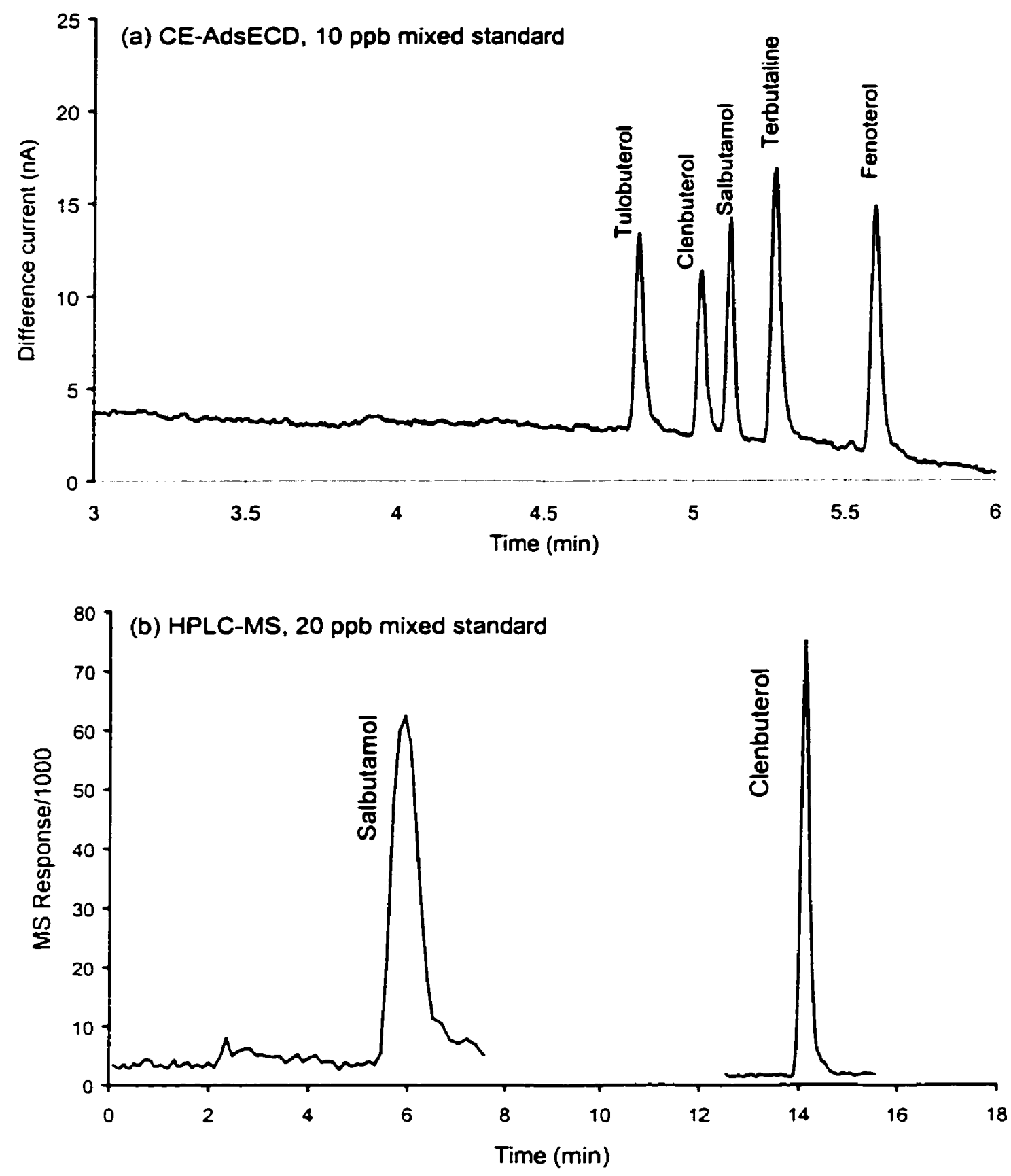

Figure 3. 24-CE-AdsECD (a) separation of $\beta$-agonists compared to LC-MS-MS (b) separation. CEAdsECD conditions described in Figure 3.23. Injection of $2 \mathrm{~s} @ 10 \mathrm{kV}$ of $10 \mathrm{ppb} \beta$-agonists prepared in water. HPLC-MS-MS conditions: VG-BIO-Q LC-MS-MS instrument used in multi-reaction monitoring mode with atmospheric chemical ionization, $60 \mathrm{mM}$, pH 3.7 formate buffer mobile phase, $2.1 \mathrm{~mm} \times 150$ $\mathrm{mm}$ Waters symmetry C-8 column. HPLC-MS-MS traces in plot (b) are the single daughter ion intensities for salbutamol and clenbuterol. 
Another important experimental parameter investigated in this study was the effect of the addition of organic modifiers to the electrolyte. Modifiers, such as methanol and surfactants, are often used to improve CE separations, and such compounds could have serious effects on AdsECD. In the separations shown in Figure 3.23, methanol was added to the electrolyte to reduce the EOF, and thus allow the analytes to remain in the capillary longer to improve the separation. Although $15 \% \mathrm{v} / \mathrm{v}$ methanol appeared to result in optimal separation, addition of methanol in concentrations up to $30 \% \mathrm{v} / \mathrm{v}$ did not attenuate the AdsECD response. The nonionic surfactant Brij-35 was also added in concentrations of $1-5 \% \mathrm{v} / \mathrm{v}$ to affect similar EOF reduction and it, too, did not impair AdsECD detection.

\subsubsection{CE-AdsECD separation of penicillin $V$ and $G$}

Penicillin $V$ and $G$ were among the first antibiotics developed and are still used extensively to combat infection in humans and animals. Although Penicillin-G is approved for veterinary use in most countries, drug residues in the meat of treated animals can result if appropriate withdrawal times are not observed. To ensure the safety of food supplies regulatory agencies have developed HPLC methods for the detection of penicillins in animal tissue ${ }^{70}$. CE separation of these penicillins based only on differences in electrophoretic mobilities alone have not been successful and CE-UV detection limits are not sufficient for residue analysis. To separate these drugs, micellar systems have been used that exploit the differing hydrophobicity of penicillin V and $G$. As the structures of penicillin $G$ and $V$ in Figure 3.25 show, both have a sulfur atom in the +2 oxidation state (similar to methionine) as well as a phenyl group which should both produce AdsECD response. 
Penicillin-V<smiles>CC1(C)SC2C(NC(=O)COc3ccccc3)C(=O)N2C1C(=O)O</smiles>

Penicillin-G

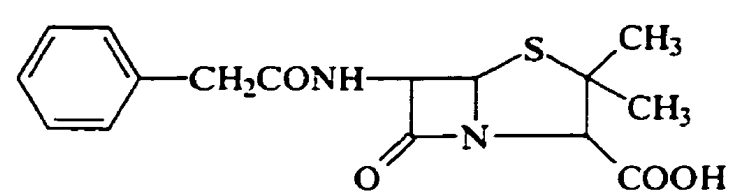

Figure 3.25 - Structures of penicillin $V$ and $G$.

A CE-AdsECD separation of Pen-G and Pen-V in a sodium dodecylsulfate (SDS) micellar system is shown in Figure 3.26. The sensitivities obtained using CE-AdsECD are slightly poorer for these penicillins than those obtained for the sulfonamide antibiotics, in spite of the fact that the penicillins have similar molecular structural features. It was determined by performing CE-AdsECD separations of the sulfonamides with and without SDS (without SDS in the electrolyte, penicillins migrate with the EOF making quantitation of the peaks difficult) that the presence of the SDS in the electrolyte attenuates the signal strength. When SDS was added to the background electrolyte, the sulfonamide peak heights were 2-3 times lower. This was likely due to two factors: $i$ ) micellar interactions with the analyte will reduce the "free" analyte available to interact with the electrode; ii) the SDS surfactant may adsorb onto the electrode, and thus compete with the analyte. Despite these slight sensitivity losses, detection limits were $-20 \mu \mathrm{g} / \mathrm{mL}$ (equivalent to $\sim 60 \mathrm{nM}, \mathrm{S} / \mathrm{N}=3$, estimated from Figure 3.26 inset electropherogram showing a $100 \mathrm{ppb}$ penicillin $\mathrm{G} / \mathrm{V}$ separation). 


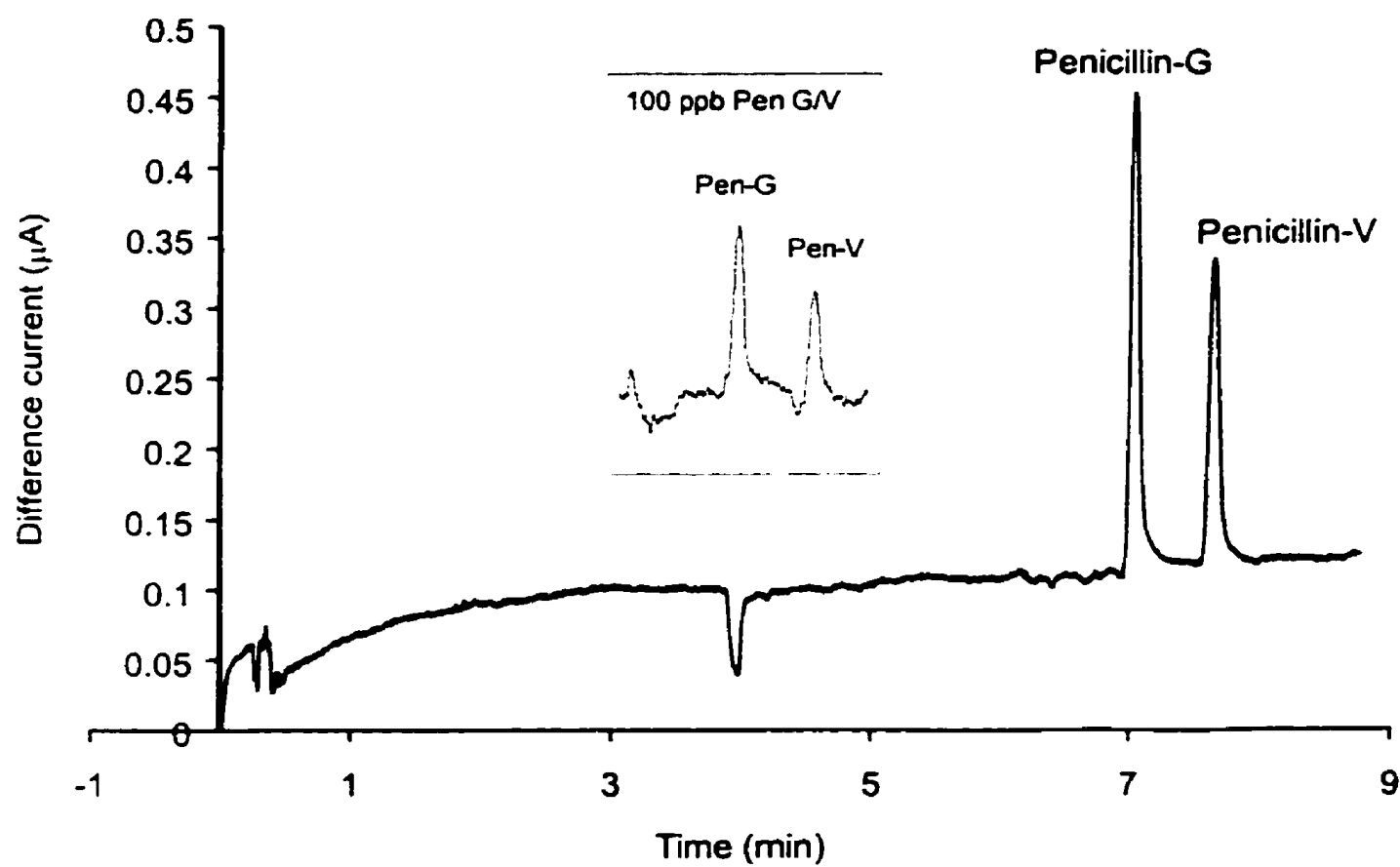

Figure 3.26 - CE-AdsECD separation of penicillins. Separation conditions: $100 \mathrm{mM} \mathrm{pH} 9.0$ borate buffer with $20 \mathrm{mM}$ SDS added, $10 \mathrm{kV}$ separation voltage, I ppm solution of penicillin $V$ and $G$ injected ( $100 \mathrm{ppb}$ solution injected in inset electropherogram). $5 \mathrm{~s} @ 10 \mathrm{kV}$. SWV conditions: DC voltage ramp : -600 to 1100 in $100 \mathrm{~ms}$ : Square-wave frequency/amplitude: $1000 \mathrm{~Hz} / 100 \mathrm{mV} ; 0 \%$ of initial square-wave current response rejected.

\subsubsection{CE-AdsECD separation of peptides}

Emergence of a biotechnology industry to support the relatively recent interest in recombinant DNA techniques has resulted in a demand for more sophisticated analytical techniques to separate and detect molecules of biological origin. Thus the AdsECD detection of peptides, an important group of biological molecules, was attempted. Polypeptides can be comprised of 100-300 amino acids linked together by peptide bonds. Figure 3.27 shows a CE-AdsECD separation of a $1 \mu \mathrm{g} / \mathrm{mL}$ solution of polypeptides. These large molecules gave similar SWV responses as the other analytes discussed previously. Sensitivities obtained using CE-UV were again $\sim 50 \mathrm{x}$ poorer than those 
obtained using CE-AdsECD. The peak tailing observed for some of the polypeptides shown in Figure 3.27 was also observed in CE-UV electropherograms.

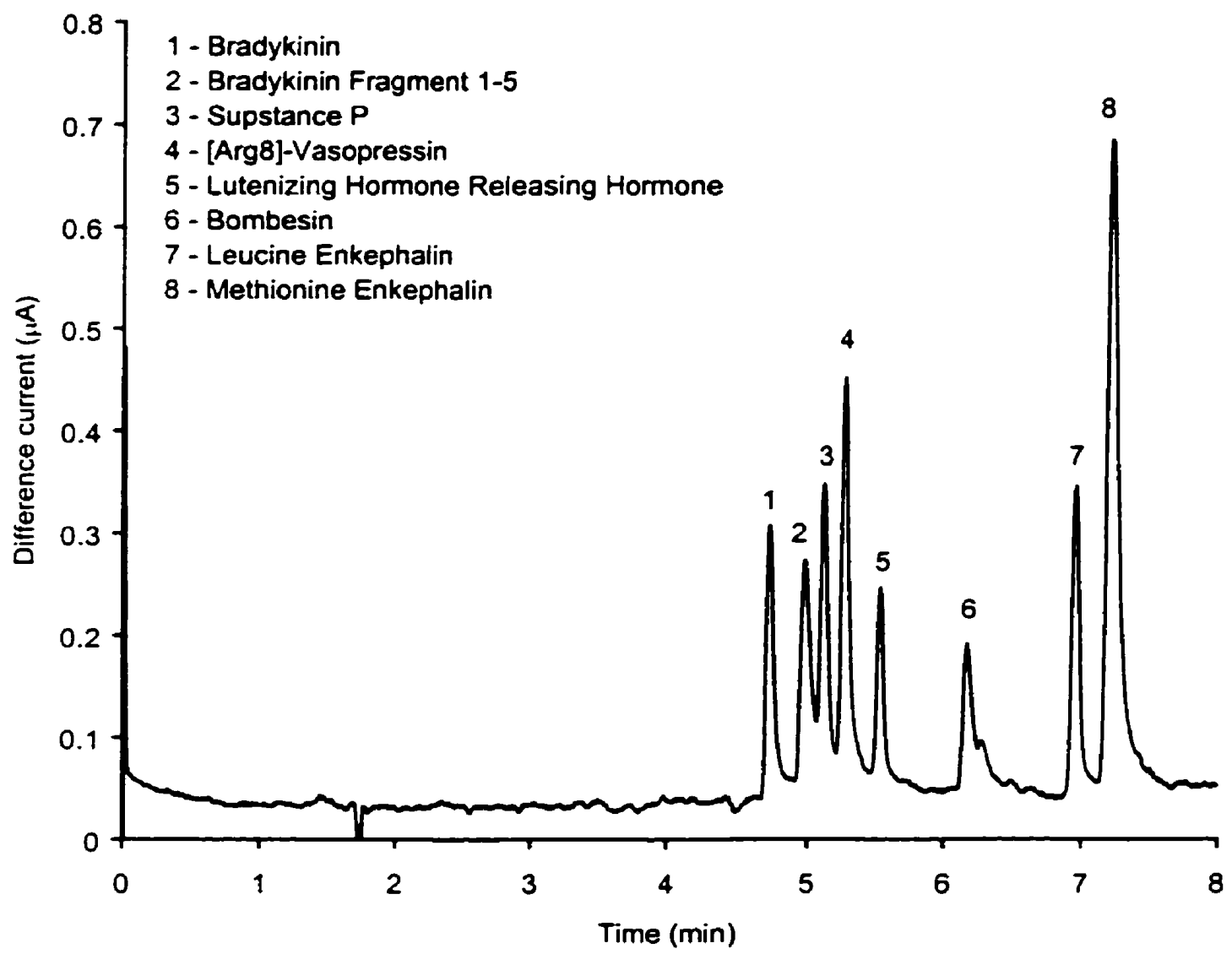

Figure 3.27 - CE-AdsECD separation of polypeptides. Separation conditions: $50 \mathrm{mM}$, pH 3.0 phosphate buffer, $10 \mathrm{kV}$ separation voltage $1 \mu \mathrm{g} / \mathrm{mL}$ solution of peptides injected, $2 \mathrm{~s} @ 10 \mathrm{kV}$. SWV conditions: DC voltage ramp : -600 to -1100 in $100 \mathrm{~ms}$; Square-wave frequency/amplitude: $1000 \mathrm{~Hz} / 100 \mathrm{mV} ; 0 \%$ of initial square-wave current response rejected.

\subsubsection{CE-AdsECD separation of nonsteroidal anti-inflammatory drugs}

Nonsteroidal anti-inflammatory drugs (NSAIDs) are a very diverse group of compounds and include many of the common over-the-counter analgesics (i.e. aspirin, ibuprofen). Although NSAIDs are not approved for use in food-producing animals, they are used for a variety of veterinary applications such as treatment of joint inflammation in 
race horses. It has been suspected that they are being used in food-producing animals illicitly to accelerate healing and minimize production losses in dairy cattle as well as to improve the presentation of slaughter animals brought to an abattoir (NSAIDs will make a sick animal "feel" better and hence appear healthy). Since treatment of this nature may result in drug residues in the food product, methods are needed for the detection of trace residues of NSAIDs in tissues of food-producing animals. Because of the possible advantages that CE may offer in the separation of this rather diverse group of compounds, a CE-AdsECD separation was attempted of four common NSAIDs suspected to be used in food-producing animals (structures of these drugs are shown in Figure 3.28).<smiles>CCCCCNc1cccc(C(F)(F)F)c1C</smiles>

Phenylbutazone :<smiles>CCCCC1C(=O)N(c2ccccc2)N(c2ccccc2)C1=O</smiles>

Diclofenac :<smiles>O=C(O)c1cccnc1Nc1c(Cl)cccc1Cl</smiles>

Oxyphenbutazone :<smiles>CCCCC1C(=O)N(c2ccccc2)N(c2ccccc2)C1=O</smiles>

Figure 3.28 - Structures of non-steroidal anti-inflammatory dnugs.

Because of the multiple phenyl rings in these compounds, a good CE-AdsECD response was expected. While flunixin and diclofenac could be separated by free-zone CE at higher $\mathrm{pH}$ where they are negatively charged, phenyl butazone and oxyphenbutazone 
are neutral at this $\mathrm{pH}$ and would migrate with the EOF, unseparated. Experiments showed that a micellar system similar to that used to separate the penicillin antibiotics (Figure 3.26) could be used to separate these analytes and a micellar CE-AdsECD separation of the NSAIDs is shown in Figure 3. 29. Similar to what was observed for the penicillin antibiotics, lower sensitivities were obtained using a micellar system for the NSAIDs as well. Detection limits of $\sim 200 \mathrm{ppb}(\sim 700 \mathrm{nM}, \mathrm{S} / \mathrm{N}=3$, estimated from response observed in Figure 3. 29) were obtained. This response was about an order of magnitude poorer than that obtained for the penicillins using a similar separation electrolyte. Although a good response was expected for the NSAIDs due to there aromaticity, the sulfur atom $(+2$ oxidation state) in the penicillins may be the reason for their stronger response.

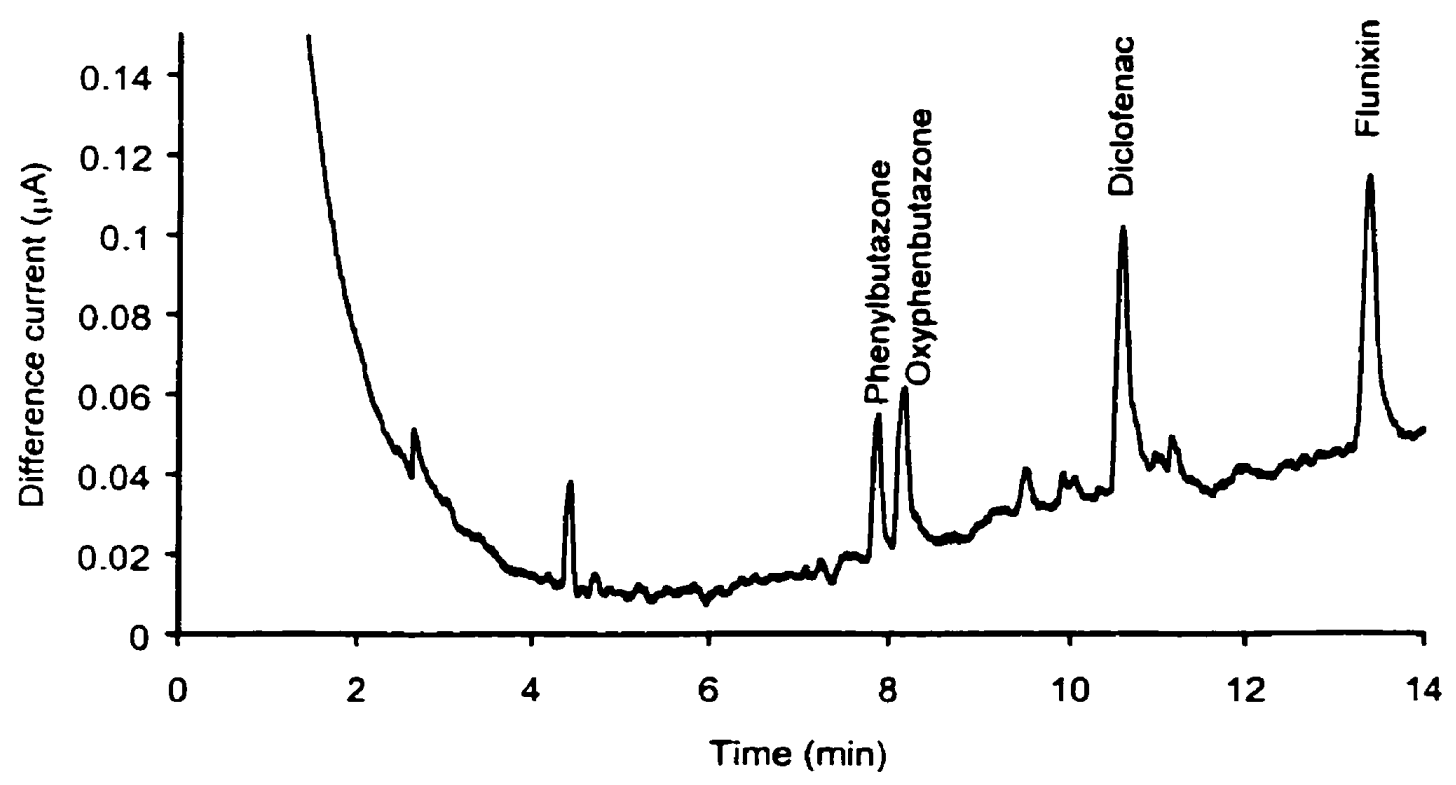

Figure 3. 29-CE-AdsECD electropherogram of non-steroidal anti-inflammatory drugs. Separation conditions: $100 \mathrm{mM}, \mathrm{pH} 9.0$ borate buffer with $20 \mathrm{mM}$ SDS added, $10 \mathrm{kV}$ separation voltage. $1 \mathrm{ppm}$ solution of NSAIDs injected, $7 \mathrm{~s} @ 10 \mathrm{kV}$. SWV conditions: DC voltage ramp : -600 to $-1100 \mathrm{in} 100 \mathrm{~ms}$; Square-wave frequency/amplitude: $1000 \mathrm{~Hz} / 100 \mathrm{mV} ; 0 \%$ of initial square-wave current response rejected. 


\subsubsection{Linearity of the AdsECD response}

Calibration curves and sensitivity plots for both the sulfonamide and $\beta$-agonist analyte groups were developed to demonstrate the linearity of the AdsECD method. For the sulfonamide antibiotics, five calibration curves were prepared over three days. Typical calibration curves and sensitivity plots obtained for the sulfonamides are shown in Figure 3.30 and Figure 3.31 respectively.

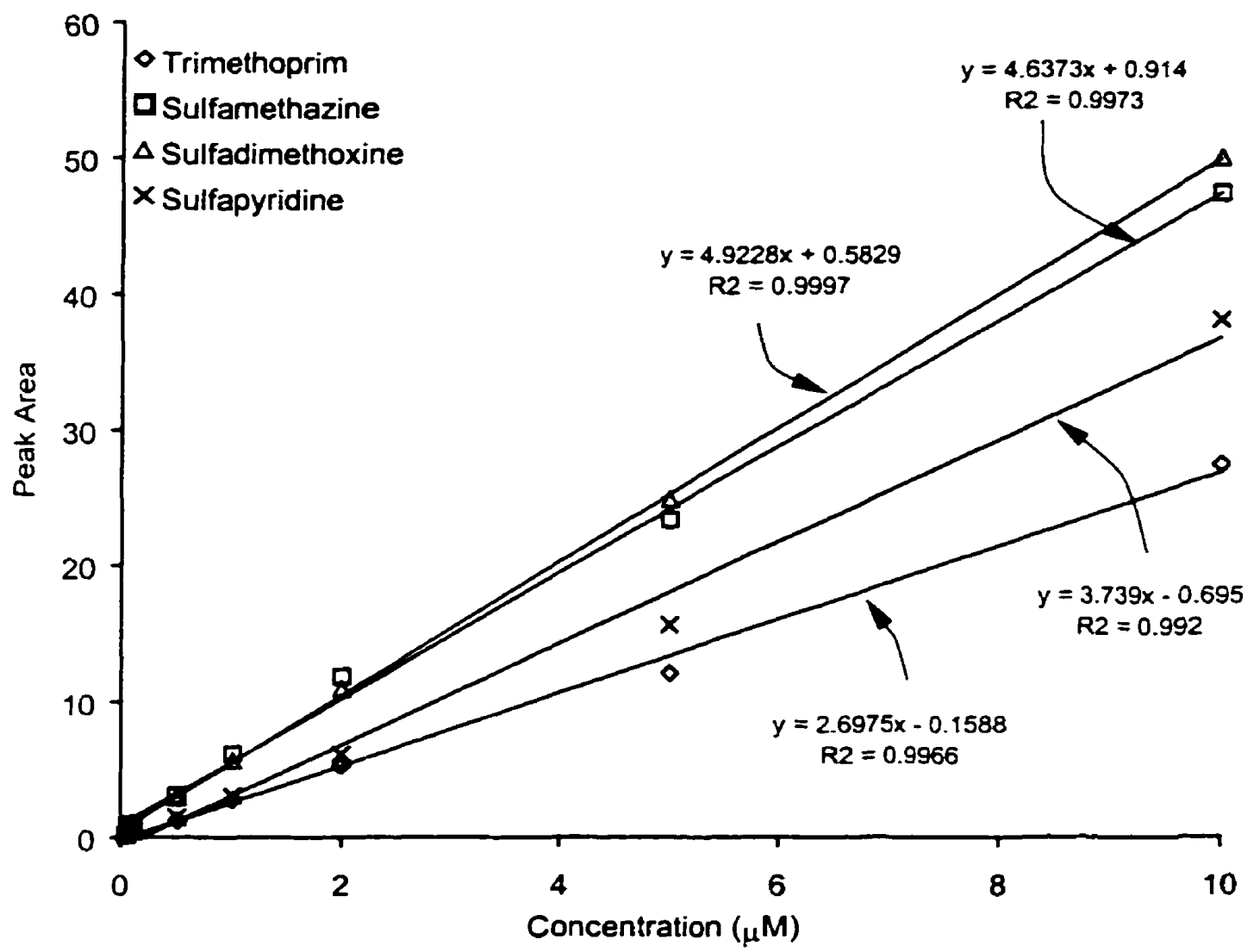

Figure 3.30 - Calibration curves for the sulfonamide antibiotics. Separation conditions used are described in Figure 3.6

As can be seen, linear regression correlation coefficient $\left(r^{2}\right)$ values were consistently above 0.997 . Linearity of the calibration curves was also tested by plotting 
the response factors (peak area/analyte concentration) versus analyte concentration, and the linearity determined by this more critical testing method was found to be reasonable ( \pm $20 \%$ ) between $5 \times 10^{-8} \mathrm{M}$ and $1 \times 10^{-5} \mathrm{M}$.

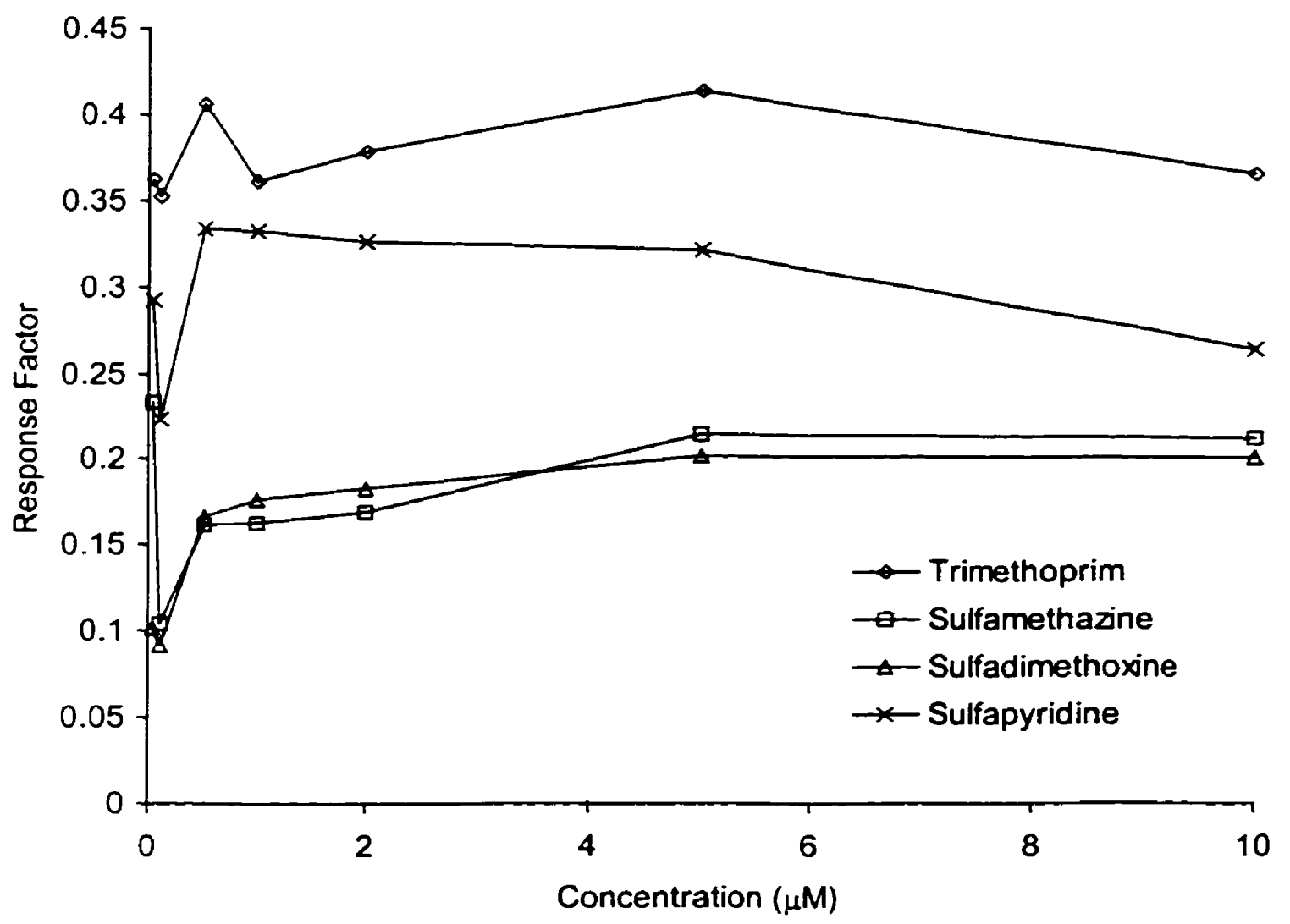

Figure 3.31 - Sensitivity plots for the sulfonamide antibiotics. Separation conditions used described in Figure 3.6 
The deviations from linearity at low concentrations shown in Figure 3.31 are almost always present in analytical calibrations, but are never observed with conventional least-squares calibration curves such as those in Figure 3.30.

Calibration curves prepared for the $\beta$-agonist drugs were performed using terbutaline as an internal standard. Peak height ratios of the other $\beta$-agonist drugs were plotted versus concentration to obtain calibration data and resulted in linear regression $r^{2}$ values consistently above 0.999 (Figure 3.32).

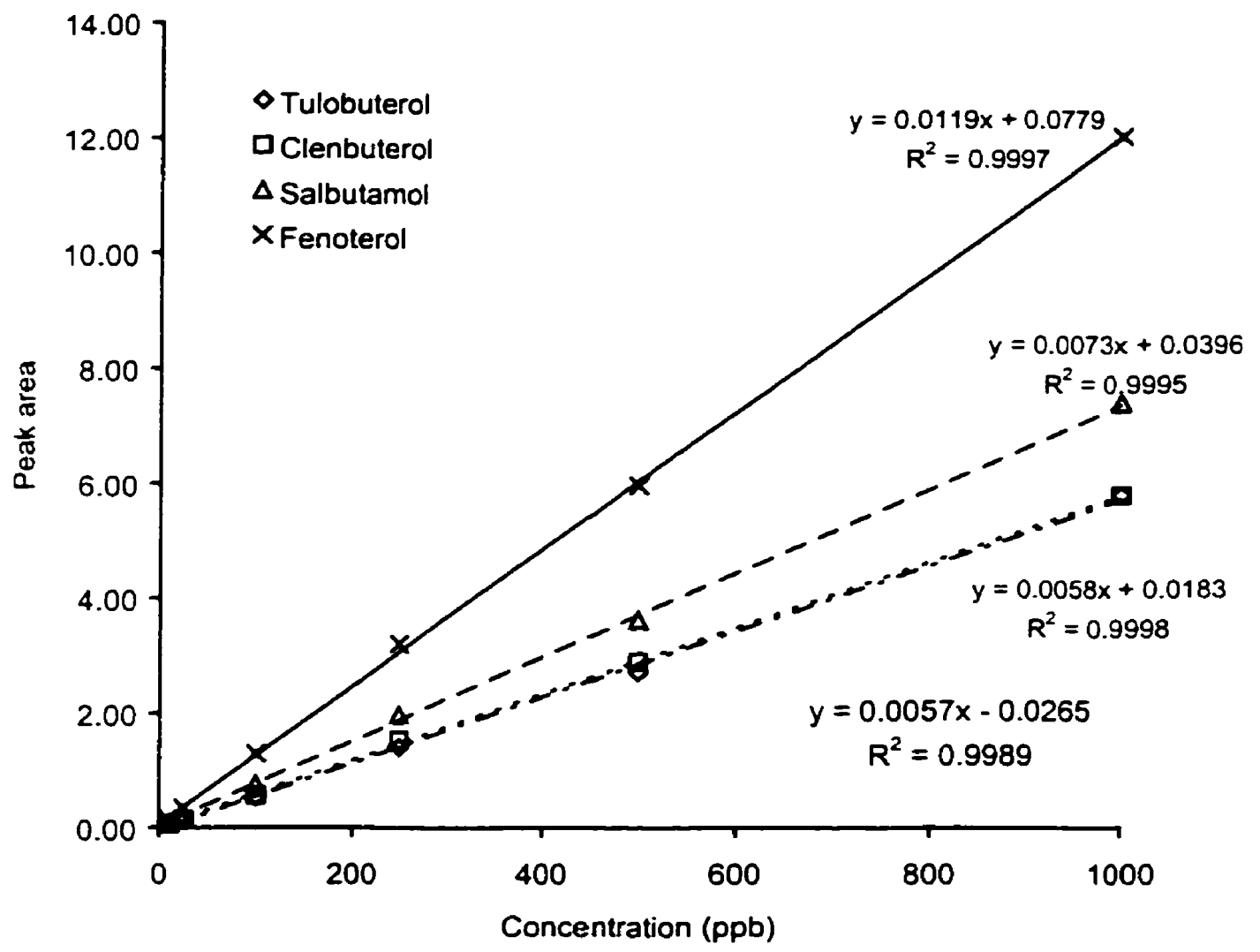

Figure 3.32 - Calibration curves of the $\beta$-agonist drugs. Separation conditions used are described in Figure 3.23 . 
Response factor plots showed excellent linearity for the concentration ranges examined (Figure 3.33). The improved linearity of the $\beta$-agonist calibration data is likely due to the use of an internal standard which compensates for both injection irreproducibility as well as electrokinetic injection bias which may occur when the standard concentration may contribute to the overall conductivity of the injection matrix. This calibration data obtained for the sulfonamides and $\beta$-agonist drugs illustrates that the adsorption-based detection method reported here has a linear response.

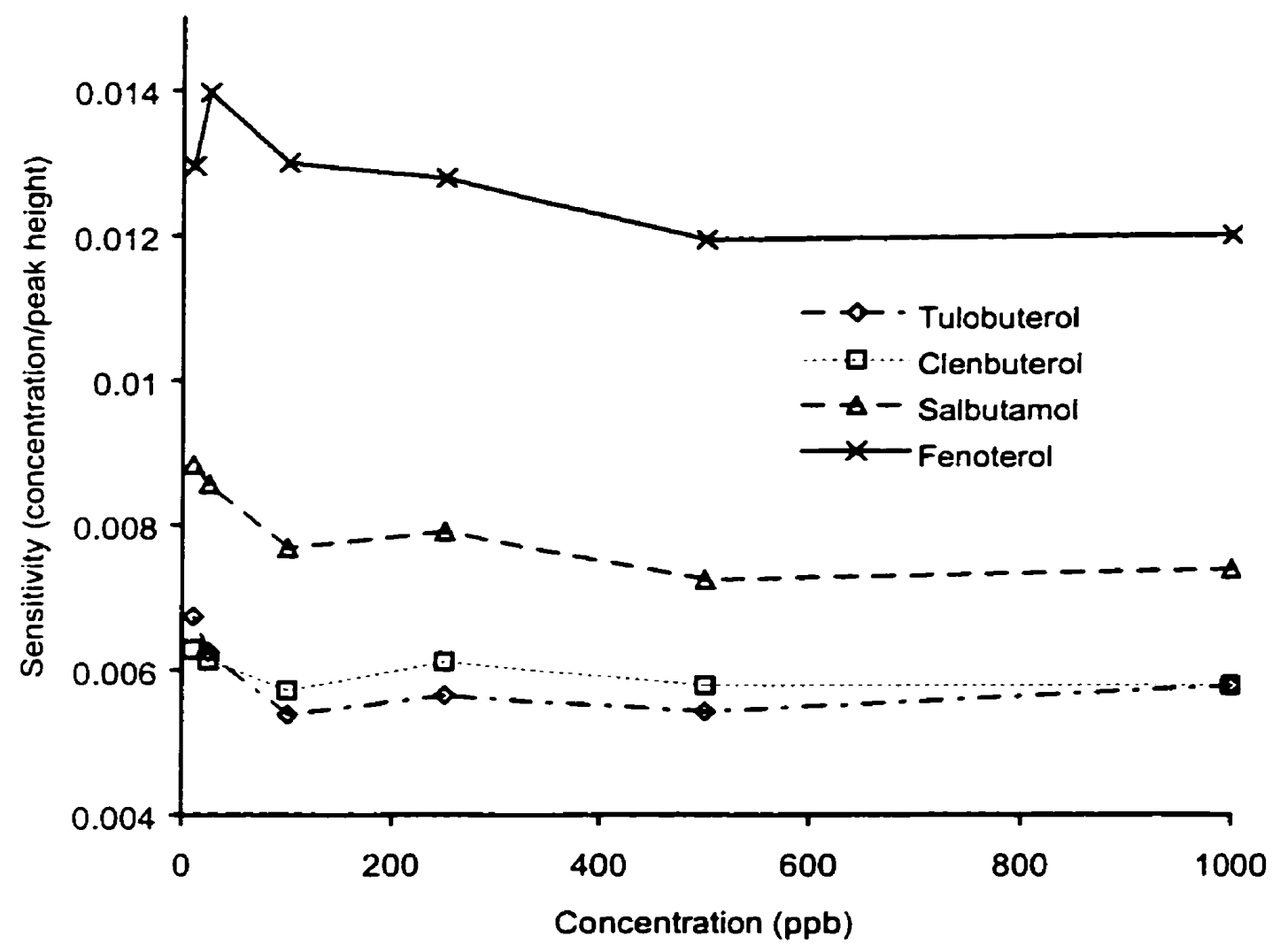

Figure 3.33 - Sensitivity plots of the $\beta$-agonist drugs. Separation conditions used are described in Figure 3.23 . 


\subsection{Conclusion}

The relatively unexplored use of $\mu \mathrm{m}$-scale electrodes to probe extreme potential regions has been exploited to scan into negative potential regions previously considered to be restricted for $\mathrm{Pt}$ electrodes because of to hydrogen and oxygen adsorption/reduction. This study has demonstrated the applicability of a new, sensitive and widely applicable electrochemical-based detection method for CE. Based upon the nature of the SWV waveform which elicits this response and the type of analytes that have responded, it appears that a complex analyte adsorption process, which may include decomposition of the analyte is responsible for the positive SWV response observed. Analytes with some degree of "available" electron density in the form of resonance-stabilized or non-bonding valence electron density give higher sensitivity with this detection method. Sensitivities obtained using this method were at least an order of magnitude better than those found using UV detection. Unlike pulsed-amperometric detection, the only other more generally applicable ECD method, AdsECD should be more widely applicable, and suitable for electrolytes over a wider range of $\mathrm{pH}$, ionic strength and buffer additives. A common drawback to electroanalytical methods has been the complexity in their application. Not only are highly trained analysts required, but due to problems such as electrode fouling, routine electrochemical detection is troublesome. With the instrumentation developed in this work, the detection method reported here is significantly simpler, more reliable and easier to implement than other electrochemical detection methods. The reasons for this are: 
- The types of analytes that respond to this detection method are easier to identify.

- Although application of this method requires rather complex data acquisition/control instrumentation (Appendix A), similar SWV waveform and current sampling conditions can be used for most analytes which respond to this detection method. Thus it should be possible to "black box" this instrumentation in such a way that its application does not require special training other than that normally required for optimization of instrument performance via software controls.

- Electrode fouling was extremely rare in the application of this method due to the high-voltage post-scan pulses and waveforms which can be optimized for those situations that offer unique matrix problems. The disk electrode design also helps minimize the severity of this possible problem, since it is easily cleaned.

- The CE-ECD cell developed in this report (Chapter 1) provided ruggedness and ease-of-use previously unavailable in the research systems used for CE-ECD systems and illustrates that better designed instrumentation can be developed for routine analytical applications. 


\section{THESIS CONCLUSION}

The purpose of this thesis was to address the limitations of current CE-ECD systems with the goal of developing a sensitive and widely applicable CE detection system suitable for routine diagnostic use. Robust, compact and easy to use CE-ECD instrumentation was developed to enable the investigation of SWV detection to CE. The traditional Osteryoung SWV method was adapted and optimized to enable the use of highspeed sweep-rates to obtain the data acquisition rates demanded by CE. Application of this CE-SWV to the separation and detection of neurotransmitters demonstrated that detection limits comparable to those obtained using more complex and fragile offcapillary amperometric detection systems were possible. The CE-SWV method was also used to investigate the possibility of using the changes which occur in the SWV response due to the adsorption of organic molecules onto the electrode as a detection method. For organic analytes without any aromaticity or other sources of $\pi$-electron density, interference of normal background SWV responses due to the reduction/adsorption of hydrogen were observed. When this response was plotted versus migration time negative analyte peaks were observed. When aromatic and sulfur ( +2 oxidation state) containing compounds were examined, positive SWV responses were observed that appeared to result from more complex chemisorption processes. This adsorption-based detection method was applied to a wide variety of analytes separated using a variety of $\mathrm{pH}$, ionic 
strength, organic additive, and micellar systems. These studies have shown that it is, indeed, a widely applicable and sensitive detection method.

There are several areas of this detection method that would be interesting to explore further:

- Develop a better understanding of the process that produces the positive SWV response for analytes with resonance-stabilized $\pi$-electron density. Techniques such as impedance spectroscopy may be useful to determine the source of this response.

- Work done late in the Ph.D. project, after the potentiostat system had been redesigned to prevent saturation of the current follower op-amp and drifting of the working electrode potential, indicated that the formation of $\mathrm{Pt}-\mathrm{OH}$ layer on the electrode surface was important in AdsECD. This should be explored further, and the AdsECD waveform re-optimized based upon this new information.

- Apply CE-AdsECD to real samples (e.g., samples extracted from environmental, biological systems). Because AdsECD appears to be sensitive to a wide variety of compounds, its lack of specificity, while an advantage in many respects, may be a limitation when attempting to separate the response of analytes of interest from the sample matrix.

- Investigate further the types of analytes which respond to AdsECD. 
- Determine the cause of the higher baseline noise observed when low $\mathrm{pH}$ electrolytes are used and determine if SWV waveform modifications could be made to minimize this effect.

- Apply AdsECD to HPLC and capillary electrochromatography. Because good sensitivities were obtained for CE separations where organic solvents were added to the electrolyte, it would be expected that AdsECD would be an effective detection method for HPLC, particularly capillary LC where poor UV absorbance detection sensitivities similar to CE have limited its application. Even for normal-bore HPLC, AdsECD may provide an inexpensive and compact detection alternative to UV absorbance detection.

- For AdsECD to be used by technicians with limited training in electrochemical techniques, the entire system must be "black-boxed." The current data acquisition/control system used in this research allowed complete flexibility, but would be too complex for routine practical application. This system should be redesigned to simplify this interface. Because it appears that the waveform and data acquisition parameters for the application of AdsECD to a variety of analytes/electrolytes are very similar, it should be possible to develop a general set of detection parameters that would reduce, significantly, the number of variables. 
APPENDIX A - DEVELOPMENT OF DATA ACQUISITION/CONTROL SYSTEMS

Author's note: This appendix describes the development of the electronic and computerbased systems used to perform the research described in the preceding chapters. This discussion is included for those who are interested in this aspect of the research. To limit the length of this section. only limited background information regarding basic electronics and computer programming is given.

\section{A.1 Introduction}

Because no commercially available instrumentation was available to perform $\mathrm{SWV}$ at the frequencies required to obtain data acquisition rates required by $\mathrm{CE}$, appropriate instrumentation was developed. The instrumentation used in this research evolved through several stages, normally occurring when the needs of the research project became more sophisticated, and as increased computing power also became available. The data acquisition/control (DAC) requirements to implement computer-controlled amperometric detection in a flowing system are, conceptually, relatively simple. A digital-to-analog (D-to-A) converter is required to generate a voltage that can be supplied to the potentiostat (to control the potential of the working electrode), and an analog-todigital (A-to-D) converter is required to sample the voltage output of the current-tovoltage converter/amplifier connected to the working electrode. If the researcher can 
manually set the potential of the electrode, there are many commercially available chromatographic data systems that can acquire, plot and quantitate the incoming current data. In voltammetric detection, however, the potential of the working electrode is scanned across a potential range while synchronously collecting current data (current samples must be collected at specific points in the potential scan). If a moving sample peak must be characterized, then this current response data be interpreted to obtain a single quantitative data point.

SWV is a powerful voltammetric technique originally used with a dropping mercury electrode (DME). The primary advantages of SWV are its speed and sensitivity. The SWV activation waveform consists of a small amplitude square-wave of period $T$ superimposed on a DC voltage ramp. The forward current is measured at time $T / 2$ and the reverse current at $\mathrm{T}$, and the difference current (forward current - reverse current) voltammograms are peak-shaped and relatively easy to obtain quantitative information from. In order to extend traditional SWV to DC scan rates higher than the technique was originally developed for (to obtain necessary overall data acquisition rates required by $\mathrm{CE})$, a DAC system was required that would not only allow complete flexibility in setting all the square-wave voltammetry parameters, but also all collection of the current samples across the entire square-wave period rather than only at the end of the forward and reverse pulses. Interpretation of this current data could then determine what portion of the squarewave response gave the best overall $\mathrm{S} / \mathrm{N}$ in the presence of high charging currents. SWV application with these requirements requires cooperation of the hardware DAC system which interfaces with the potentiostat and current follower/amplifier with a software 
application to control the DAC as well as interpret the current response and generate an output for the user interface. The initial systems that were developed for this research were based upon DAC cards inserted in a Wintel (desktop computer using a Intel x86 CPU and Microsoft Windows operating system) machine. While these systems performed well, it was later determined that equivalent performance could be obtained using microcontrollers (single-chip CPU with built-in VO interface) which were much more cost effective, and compact. This appendix will briefly describe these earlier PC card-based systems and discuss in more detail the current microcontroller-based DAC system used for CE-SWV.

\section{A.2 PC card-based DAC systems}

The initial DAC PC card that was used was the PCL-812 Enhanced Multilab card (B\&C Microsystems Inc.). This board allowed a relatively fast A-to-D data acquisition rate or $30 \mathrm{kHz}$, at 12-bit resolution and a 12-bit D-to-A port with $30 \mu$ settle time. Initial applications were developed with Borland Delphi v.1.0 programming language (Borland International), and run with Windows 3.1. Development of PC-based DAC systems on a Wintel machine are hindered by Microsoft Windows control of hardware interrupts. To apply a high-speed waveform while synchronously collecting current data, a Windows DAC application must be able to have unfettered access to hardware timers and interrupts. This limitation was overcome by using timers residing on the PCL-812 card to control the timing of the waveform output rather than the PC-based timers that are used by the Windows operation system. To perform data acquisition under Windows, handling of the PC interrupts had to be shutdown during each voltammogram scan and re-enabled after 
the scan had completed. Also, to obtain the necessary computing speeds to perform the timing sensitive SWV scans, assembly language routines were used inline with the Delphi code.

Listing A.1 illustrates the assembly language routine used to apply a SWV scan. For readers with little knowledge of assembly language programming, the general flow of this routine can be understood by reading the bracketed comments to the right of the code. As can be seen, each time this procedure is entered, the CLI command is given to the CPU to shutdown interrupt handling, and the STI command is issued after the scan has completed to resume interrupt handling. The waveform that was applied using this routine was stored in a buffer. This allowed the same routine to be used for a variety of voltammogram waveforms. The current data were sampled at the maximum data acquisition rate and spooled into a buffer using direct memory access (DMA). After each SWV scan, the current response was interpreted and plotted versus time to generate an electropherogram. During the small pauses between each SWV scan, the electrode remained at the last potential stored in the waveform buffer, which did not appear to cause problems. Although this system worked well, this direct control of hardware was not accepted in the newer Microsoft Windows NT 4 operating system. Under Windows NT 4, direct control of hardware is only possible by writing device drivers, but this is a rather involved procedure. Consequently, the National Instruments AT-MIO-16E board was used, as it had similar features as the PCL-812 card, but had a documented library of routines which enabled background DMA transfers and synchronous buffered waveform application. Whereas in the PCL-812 card synchronous waveform application and data 
acquisition was performed using assembly language routines, the National Instruments card library functions offered a much more elegant interface that did not require any manipulation of the interrupts. Although this software/hardware system offered no significant improvement in the basic performance of the SWV analysis, other advantages were achieved because the software could now be run under Windows NT 4.0 which allowed much faster access to a larger 32-bit memory space.. Also, Windows NT 4.0 had the capability to run using multiple processors. A dual Pentium Pro (200 MHz) system was used that allowed division of the data acquisition as a separate task ${ }^{\mathrm{I}}$. A screenshot of this application is shown in Figure A.l. As can be seen, this program system offered considerable flexibility and allowed a variety of voltammetric and amperometric methods (square-wave, cyclic, and linear scan voitammetry as well as DC and pulsed amperometric detection) for CE detection. Figure A.1 also shows that having obtained the detection data from the electrochemical response, the application plotted the electropherogram data as well as identified and integrated any peaks in the electropherogram.

\footnotetext{
'Windows NT 4.0 allows applications to split processes into separate tasks. A multi-task application will then take advantage of multi-processor machines by assigning a high-priority task to a specific processor, allowing the other, lower priority tasks to be maintained by the second processor thereby reducing the overall lag of the application due to a single high-priority task.
} 


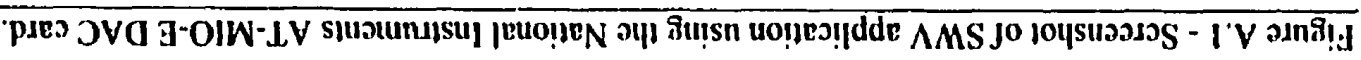

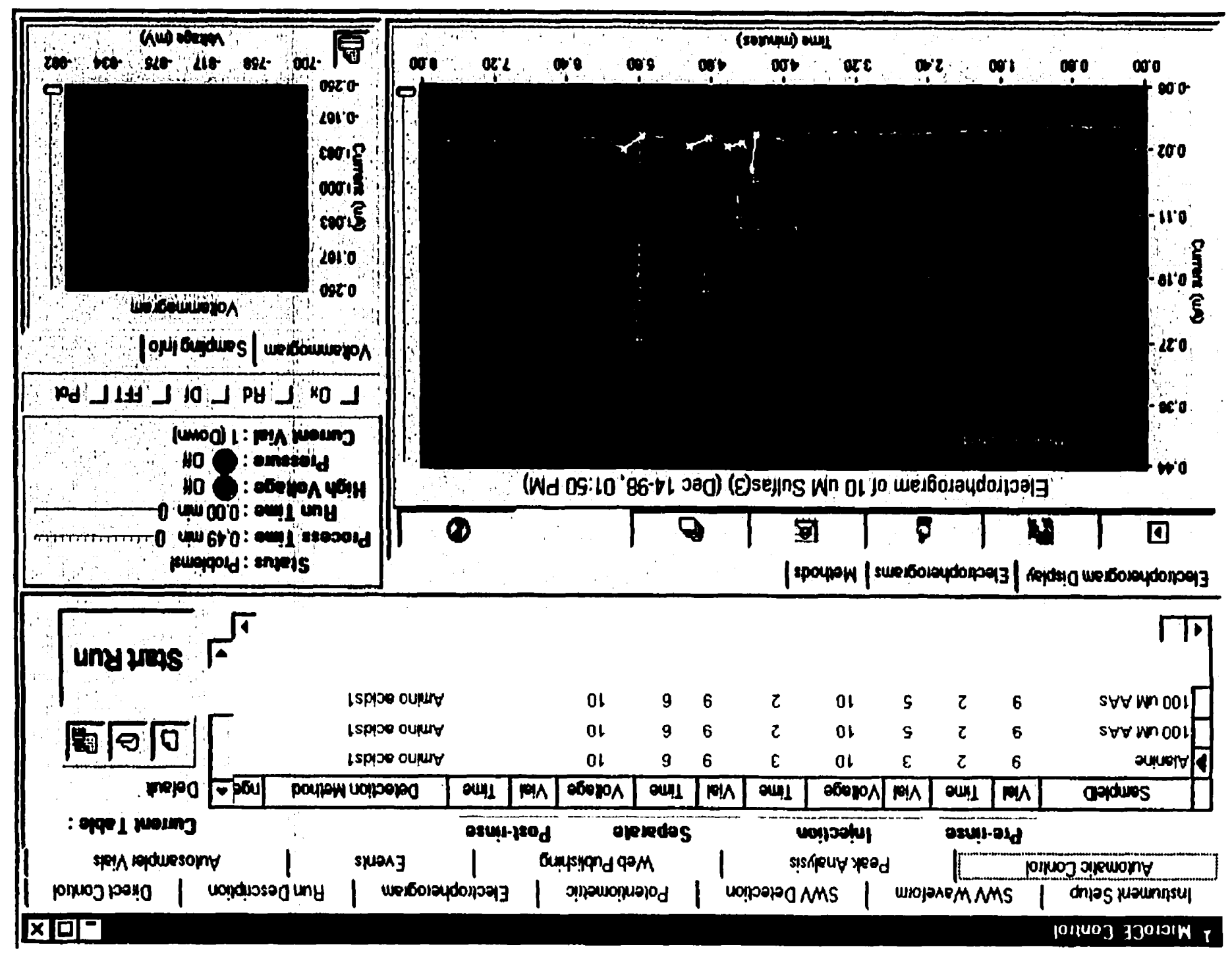




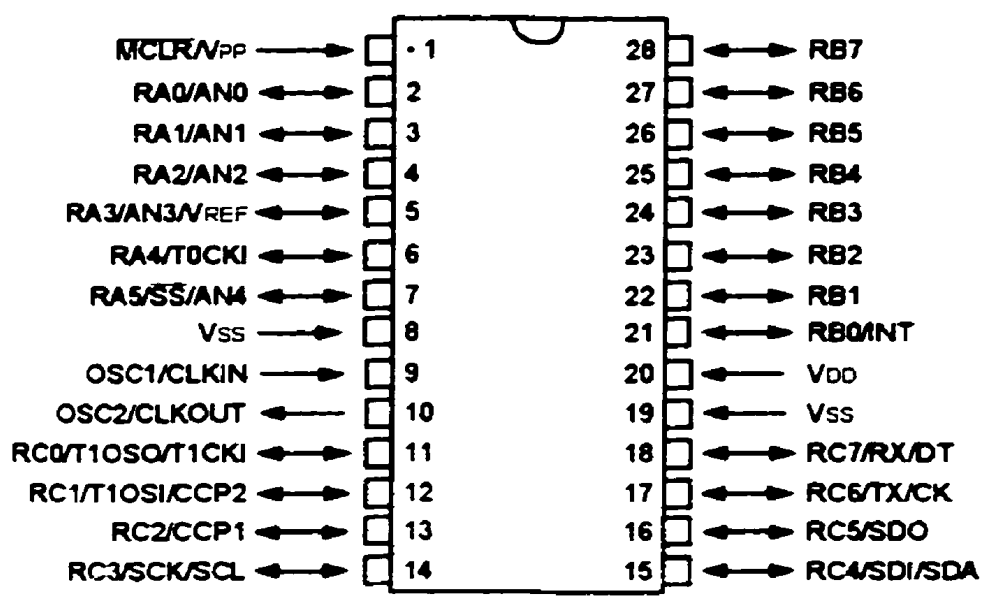

Figure A.2 - Pin-out of 16C73 PIC microcontroller.

\section{A.3 Microcontroller-based DAC systems}

One problem encountered with the PC-based DAC card SWV system was it required a relatively powerful PC because the data acquisition process demanded a significant portion of the PC CPU time. In addition, the DAC cards themselves were rather expensive (>\$2000 CDN, 1998). An altemative is the use of microcontrollers which are single-chip computers that have RAM, ROM (program memory), digital LO, Dto-A conversion and serial communications built-in. In contrast to a CPU in a desktop PC, they run at relatively slow speeds (typically 4-20 MHz). However, because they do not have any operating system overhead, they can execute specific tasks, particularly timing sensitive tasks more efficiently than PC-based DAC systems. In addition, an entire microcontroller system can be assembled for $<\$ 50$ (CDNS, 1998). A microcontroller system could be designed to manage the DAC functions, thus relieving the host PC application of most real-time responsibilities that are made difficult under the modern Windows operating systems. It was because of these advantages that development began 
on a microcontroller-based CE-SWV DAC system. The microcontroller that was used was the 16C73 PIC (Microchip Technology Inc., Chandler, AZ, U.S.A.). The pin-out of this chip is shown in Figure A.2

Table A.1 - Description of pin assignments used on PIC16C73

CE-ECD Function PIC Functional Name PIC Pin

$\begin{array}{rll}\text { ECD analog in } & \text { A0 } & 2 \\ \text { UV analog in } & \text { A1 } & 3 \\ \text { D-to-A Clock } & \text { A2 } & 4 \\ \text { Spare analog in } & \text { A3 } & 5 \\ \text { Not used } & \text { A4 } & 6 \\ \text { D-to-A A1 } & \text { A5 } & 7 \\ \text { Stepper shift register Serln } & \text { B0 } & 21 \\ \text { Stepper shift register clock } & \text { B1 } & 22 \\ \text { Stepper shift register latch } & \text { B2 } & 23 \\ \text { HV Polarity (HI=-ve, LO=+ve) } & \text { B3 } & 24 \\ \text { Current follower amp gain A0 } & \text { B4 } & 25 \\ \text { Current follower amp gain A1 } & \text { B5 } & 26 \\ \text { Not used } & \text { B6 } & 27 \\ \text { Not used } & \text { B7 } & 28 \\ \text { Working electrode on/off } & \text { C0 } & 11 \\ \text { D-to-A Data } & \text { C1 } & 12 \\ \text { Not used } & \text { C2 } & 13 \\ \text { Speaker } & \text { C3 } & 14 \\ \text { Stepper stop } & \text { C4 } \\ \text { HV Enable } & \text { C5 } & 15 \\ \text { RS-232 connection to host } & \text { C6 } & 16 \\ \end{array}$


This microcontroller has three banks of $\mathrm{IO}$ ports that can be configured as digital input, digital output or analog input (only ports A0-5 can be configured for analog input). Table A.1 describes the port usage of the PIC16C73 for the automated CE-ECD system.

\section{A.3.1 Programming the microcontroller}

The PIC $16 \mathrm{C} 73$ microcontroller has $4 \mathrm{~Kb}$ of ROM for program storage. With a specially designed microcontroller programmer (EPIC, microEngineering Labs, Inc., Colorado Springs, CO, U.S.A.) programs can be downloaded from a PC to the microcontroller. Although the program downloaded to the microcontroller must be in the form of byte-code machine language, several assembly and natural language compilers are available to generate byte-code. After a review of the available compilers, it was determined that the PIC-C compiler (CCS Inc., Brookfield, WI, U.S.A.) provided the most flexibility and allowed timing-sensitive routines to be programmed. Although PIC-C follows ANSI-C quite closely, a number of library functions were added to allow relatively easy access to microcontroller functions. An RS-232 serial data link was established between the host PC Windows application and the microcontroller that was used to transfer waveform information from the host application to the microcontroller as well as sampling current data from the microcontroller to the host. On the PC-based DAC systems the waveform was created and stored as a sequence of numbers in a buffer. This was mainly done to synchronize waveform application with current data sampling. In the PIC DAC system, it was easier to create the waveforms "on-the-fly." The SWV waveform requested by the user in the Windows host application was processed and converted into pertinent timing and voltage specifications (DC ramp initial potential, step 
height and number of steps, square-wave period and amplitude, post- and pre-scan pulse potentials and durations, the number of initial square-wave current response samples rejected, and gain of the current follower) and sent to the microcontroller. Once the waveform information was transferred to the microcontroller, the code shown in Listing A.2 was called to perform the SWV function. All timing of the waveform generation was performed with one of the 16-bit timers built in the $16 \mathrm{C} 73 \mathrm{PIC}$. Once the timer was set to an appropriate interval time, events were timed by entering a 16-bit value in its counter register. The timer incremented this register after the timing increment and when it reached SFFFF, an interrupt was generated. An interrupt routine (not included in this code) then set the boolean "TimeriDone" variable to TRUE. After setting the timer, TimeriDone was monitored to determine when the timer had expired. Before the SWV scan was applied, two pre-pulses were applied (Listing A.2, lines 50-63). Typically, only one pre-pulse is used (the pre-pulse \#l timer is set to SFFFF to indicate it should not be applied). The square-wave was applied in two stages, the positive and negative pulses of each square-wave (Listing A.2, lines 64-107). The median value of the square-wave potential, voltout is incremented by stepAmp after each square-wave to generate the DC ramp (Listing A.2, lines 106).

After the square-wave potential was applied, the current was sampled using one of the 8-bit A-to-D ports on the 16C73 PIC (the limitations of using an A-to-D converter with 8-bit resolution is discussed in below) while waiting for the square-wave period timer to expire. The current samples collected during this time period were summed which resulted in aggregate forward and reverse currents. Initial current samples could still be 
rejected (Listing A.2, lines 82 , Reject Ist is a parameter received from the host), but total raw current response data was no longer available using this microcontroller-based DAC system, as it was in the PC-based DAC system. This is due mainly to the difficulties in transferring large amounts of data to the host PC application while still performing SWV. Immediately after each forward and reverse square-wave pulse had been completed, the 16-bit aggregate current sample was sent to the host as two bytes (the 16bit integer's high and low bytes, Listing A.2, lines 86-88). Because this data transfer must be completed as quickly as possible to avoid timing delays in the waveform application, this was determined to be the most efficient way to transfer a 16-bit integer. The accuracy of the waveform timing using this "on-the-fly" data transfer method was verified using an oscilloscope. For each SWV scan made by the microcontroller, the host $\mathrm{PC}$ will receive a string of SWV data preceded by "STR" and terminated by "END" . In addition, the number of square-wave periods in each scan along with the number of current samples acquired during each forward and reverse pulse are also encoded in each scan. Because each square-wave period will produce 4 bytes and the number of square-wave periods in each scan is known by the host program, routines were written to parse this incoming data stream to extract the current data into buffers.

\section{A.3.2 Parsing of SWV scan data sent from microcontroller}

Delphi versions 3 and 4 were used to write the host Windows application used to communicate with the microcontroller, interpret the voltammogram data, and display it as an electropherogram. Delphi, an extension of the language Pascal, includes object oriented programming. Because Pascal is one of the more natural-looking programming 
languages the reader requires only a basic knowledge of general programming concepts to understand the code listings shown here. The following is the format of the scan data that will be received by the host program while the microcontroller is running the RUNDAQ () procedure:

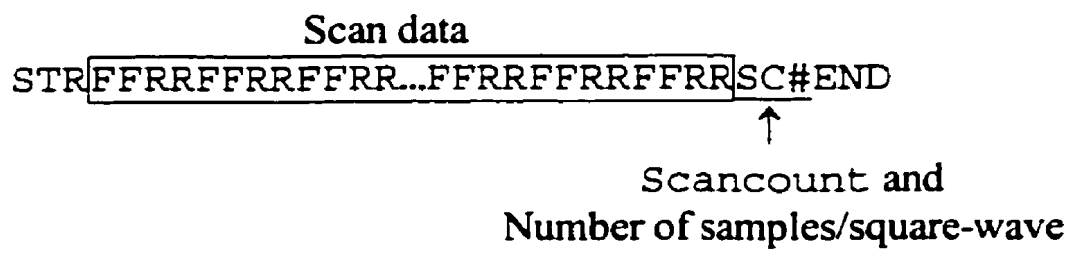

Where :STR - scan data start delimiter.

EF - 2-byte format of the aggregate current sampled across one forward square-wave pulse.

$R R \quad$ - 2-byte format of the aggregate current sampled across one reverse square-wave pulse.

SC - Scan count sent as 2-bytes.

\# - Number of samples acquired during each square-wave sent as a single byte (the number of samples/square-wave should not

exceed 255).

Incoming data from the PIC is buffered to ensure no SWV scan data are lost during times when Windows partitions CPU time to other applications. As incoming scan data is detected a communications event (not shown) adds incoming data to a string variable called ComIn. The ParsePICScan procedure (Listing A.3) is called at regular intervals by the host application to process scans contained in ComIn. If a complete SWV scan is detected in ComIn (Listing A.3, 19), the start and end of the scan data is determined (Listing A.3, 21-28). The number of samples per square-wave (NumECDSamples) and scan number (ScanNum) are extracted from the scan data (Listing A.3, 31,32). By knowing the scan number and the time required to complete each 
scan (TotaIScanTime, calculated when the waveform parameters are sent to the microcontroller), an elapsed time is calculated for the scan being processed. This elapsed time will be tagged to the resulting voltammogram buffer that will be created and will be used when the quantitated voltammogram data is displayed as an electropherogram. The position of the forward and reverse square-wave response within ComIn is identified and each aggregate current sample is extracted (as a 2-byte code), converted back to a 16-bit integer and stored in the scan array. After the current data have been placed in the array, voltammogram buffer objects are prepared to hold the forward, reverse and difference current data. Because the current is stored as the sum of $0-255$ bytes, the mean current is determined using the number of samples per square-wave extracted from the scan data and then converted into a $\mu \mathrm{V}$ value before being stored in the voltammogram buffer object. Storing the current data using $\mu \mathrm{V}$ permitted the use of a 32-bit long integer (4-bytes) rather than a float-type number (8-bytes), which reduces the overall size of the stored voltammogram. Conversion of the measured data units from voltage to current is performed when the data are displayed and is based upon the gain used on the current follower. This ParsePICScan procedure is called every $100 \mathrm{~ms}$ by the host application.

\section{A.3.3 Quantitation of the voltammogram data to produce an electropherogram} The ParsePICScan procedure will produce a list of voltammogram objects labeled with a time value to indicate their sequence and timing. A separate procedure was also called regularly to process these buffered voltammograms in order to obtain quantitative information that was displayed versus run time to generate an 
electropherogram. The Quanti fySWV procedure is shown in Listing A.4. There are several ways to integrate a SWV response. If a peak-shaped response is expected, the difference current response at the peak voltage can be used, but this will use only one point of the voltammogram. It was determined that $\mathrm{S} / \mathrm{N}$ can be increased by selecting a "bandwidth" of points centered around the maximum SWV response and averaging the current response across this region. The signal averaging obtained using this method significantly reduces the ultimate noise observed in the electropherogram. Also, because the SWV response is often peak-shaped, it was also found beneficial to use only difference current data existing within the peak itself by drawing a baseline across the voltammogram (see section 2.3 .4$, p. 78 ) and ignoring current data outside the area defined by the peak and its baseline. To allow the user to select the optimal quantitation method, the procedure shown in Listing A.4 was able to change the quantitation mode based upon user instructions. The QuantifySWV procedure was called regularly during a CE separation whenever new voltammogram data was detected. The voltammogram list could also be re-analyzed using different quantitation methods to determine the most appropriate parameters. Although the Quanti EYSWV procedure appears complex, this is only because it must be flexible enough to accommodate a variety of quantitation modes. The reader is referred to comments included in Listing A.4 which describe in general terms the mechanics of this procedure.

\section{A.3.4 Implications of using an 8-bit A-to-D converter for SWV current data collection} While the A-to-D converters used in the PC-based DAC cards had 12-bit resolution, the A-to-D converters available on the 16C73 PIC microcontroller were only 8- 
bit. Although 8-bit data acquisition resolution was initially considered inadequate, spreadsheet simulations showed that, because of the extensive signal averaging used to generate the final result in SWV, the actual resolution of the ultimate analytical signal produced by this method was much higher than 8-bits. Figure A.3 illustrates the results of these simulations. Figure A.3a shows a simulated analog signal that is to be digitized. If this signal is digitized using a 2-bit A-to-D converter, the result is the curve shown in Figure A.3b. However, if random noise is added to the signal shown in Figure A.3a, and this signal is repeatedly sampled using this same 2-bit A-to-D converter in such a way that sample is the average of 30, 2-bit A-to-D conversions, the digitized form of the original signal that will result is shown in Figure A.3b. As can be seen, the overall resolution of the digitization process has been increased by this over-sampling/averaging.

To further clarify this, consider a single voltage of $0.7 \mathrm{~V}$ that is being sampled using a 1-bit A-to-D converter with an input range of $0-1 \mathrm{~V}$. One conversion of a noisefree sample would be interpreted as a digital " 1. " Consider now, if random noise of an amplitude of $0.5 \mathrm{~V}$ were added to this signal and 10, 1-bit samples were taken and averaged. If the distribution of the noise is random, sampling probability would predict that 7 of these 10, 1-bit samples should be a digital " 1 ," and 3 should be a digital " 0. " When these aren averaged, a A-to-D conversion value of 0.7 would result. Consequently, the resolution has been increased from 2 units/1V range (1-bit resolution) to 10 units/lV range. This over-sampling/averaged enhanced resolution would be equivalent to using a single conversion from a 3.3-bit A-to-D converter (i.e. $2^{3.3}=10$ ). This A-to-D resolution 
enhancement technique must be limited to applications where signal-averaging used will not impair the frequency of the signal being measured.

To generate an electropherogram point from a SWV current response, a large number of A-to-D samples are averaged (the exact number depends upon a number of parameters). Firstly, as shown in the C code in Listing A.2 that is used to gather the SWV current response, a number of current response samples are averaged to obtain both the forward and reverse currents. These forward and reverse currents are then combined to obtain the difference current. During the quantitation of the SWV voltammogram (Listing A.3), a number of difference current samples across a potential range are averaged to obtain the final average difference current response that is plotted to generate the electropherogram. The data acquisition rate that is generated using the code shown in Listing A.2 is $44 \mathrm{kHz}$. 
(a)

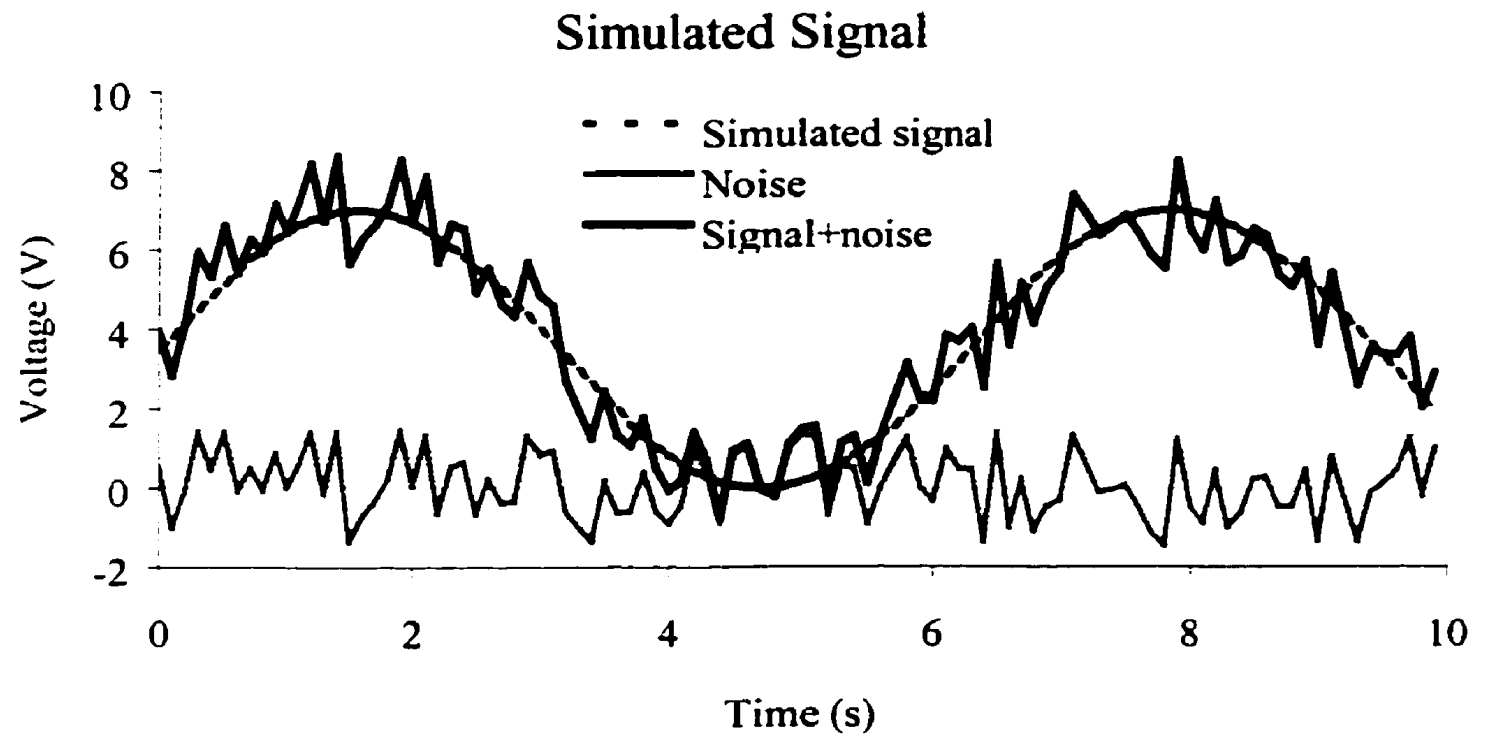

(b)

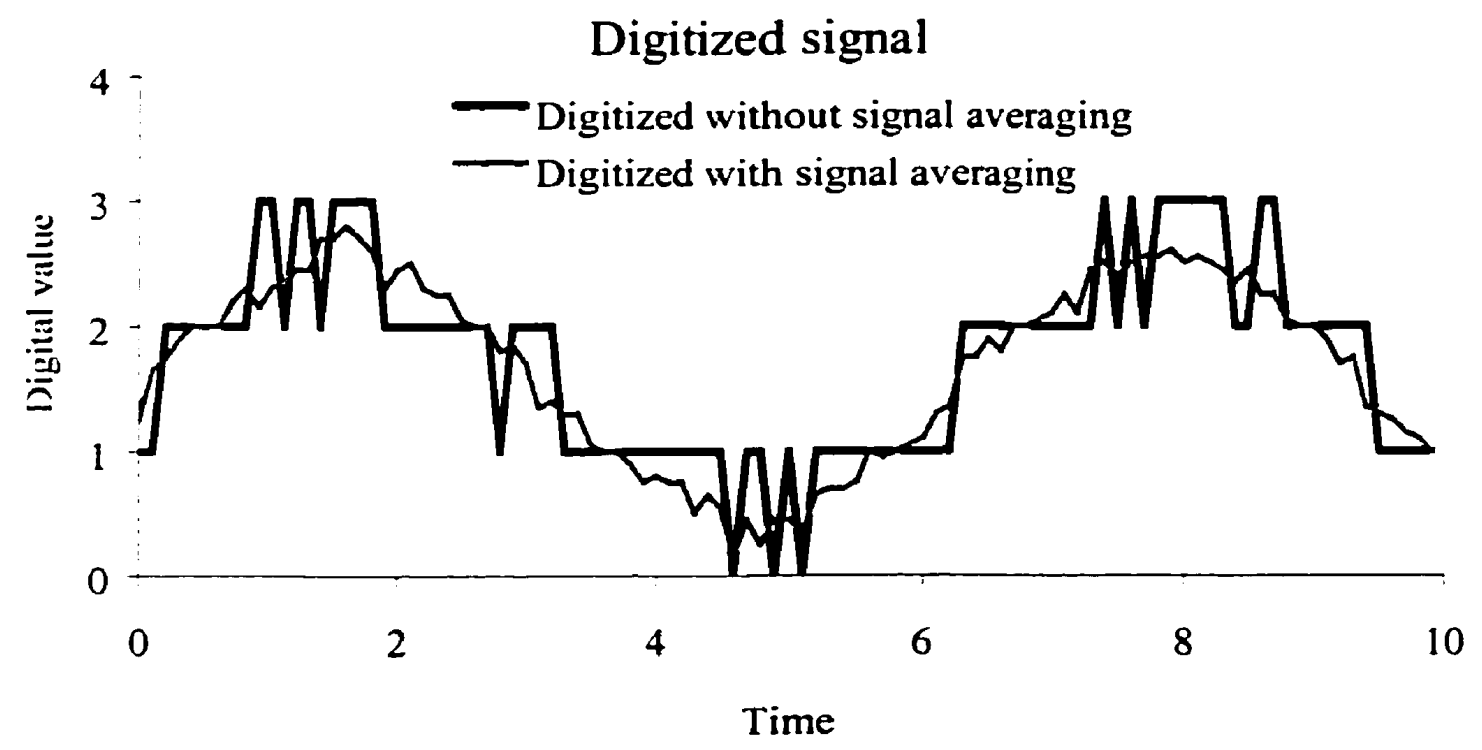

Figure A.3 - Simulations illustrating the resolution enhancements produced by signal averaging. 
If a SWV scan is completed in $100 \mathrm{~ms}$ and a $10 \mathrm{~ms}$ band of difference current samples are averaged to obtain a value for that voltammogram, then a total of 440,8 -bit samples would have been used to generate that value. This would be equivalent to a single A-to-D sample taken using a 16.8 bit A-to-D converter (i.e. $2^{16.8}=440 \times 256$ ). Even if only one point in the voltammogram response is used for quantitation, a typical square-wave period of $\sim 1 \mathrm{~ms}$ would result in 44 data points. If the first 10 points of each square-wave response were rejected, this would result in a total of 24 data points and a resolution of $\sim 12.6-$ bits (i.e. $2^{12.6}=24 \times 256$ ). Resolutions of this order are, in fact, observed in the final electropherograms generated. Although it is difficult to compare the results obtained using DAC systems developed by using PC-based cards with those obtained using the microcontroller systems, because different potentiostat designs were used with each (improvements were made to the potentiostat design used with the microcontroller systems), the overall $\mathrm{S} / \mathrm{N}$ obtained using the microcontrollers are approximately equal to those obtained using the PC-based DAC systems. As a result, this pseudo A-to-D resolution enhancement appears to allow an 8-bit hardware A-to-D converter to be used for this application.

\section{A.4 Potentiostat and current follower circuits}

The potentiostat/current follower discussed in section 1.2.4 was a functional description of the actual potentiostat/current follower circuits that were interfaced to the DAC systems. To interface the ECD electronics to the DAC system, several additions and modifications were made to the basic circuit shown in Figure 1.4. The circuit used with the most recent microcontroller-based DAC system is shown in Figure A.4. To enable digital 
selection of the current amplifier gain, a digital switch (U5) was added to the current follower op-amp (U1A). Because the range of the 8-bit A-to-D converter on the $16 \mathrm{C73}$ PIC is from 0$5 \mathrm{~V}$, a voltage adder circuit was added to offset the output of the current follower/amplifier by $2.5 \mathrm{~V}$ which resulted in a current of 0 A producing a voltage of $2.5 \mathrm{~V}$. As a result, both positive and negative current signals could be digitized. An adjustment was added to this offset (R3) to allow tuning the offset of the current response to ensure its range could be accommodated by the A-to-D converter. Because the output of the op-amp feeding the A-to$\mathrm{D}$ converter is $\pm 12 \mathrm{~V}$ and the range of the PIC A-to-D converter is $0-5 \mathrm{~V}$, voltages outside this input range needed to be clipped to avoid damaging the microcontroller. This was performed using diodes D1 and D2 to clip voltages above $6 \mathrm{~V}$ and diode D3 to prevent negative voltages.

During idle periods (i.e., when the potentiostat was not applying a waveform), the potential working electrode was held at virtual ground, and subject to the potential applied to the auxiliary electrode. Because this could lead to electrode fouling, a digital switch was used to connect the working electrode to the current follower. When the working electrode was connected to the current follower, its potential was held at virtual ground, but when this digital switch was opened, the working electrode was allowed to float. As can be seen in the microcontroller code used to apply the SWV waveform (Listing A.2), immediately before the waveform was applied the working electrode was connected to the current follower (Output_High (WorkingElectrode)). After scanning has been stopped, the digital switch was opened (Output_Low (Workingelectrode)) and the electrode was allowed to float. 
A 16-bit D-to-A converter (Burr Brown DAC614, U3) was used to output the SWV waveform to the potentiostat op-amp (U1B). In the configuration shown in Figure A.4, this D-to-A converter was able to output $\pm 5 \mathrm{~V}$ based upon a twos-complement numeric value between 0-SFFFF passed to it from the microcontroller using a serial interface. This serial interface required only 3 microcontroller ports. Adequate data transfer speed could be achieved using this serial interface and SWV waveform application was not impeded. 


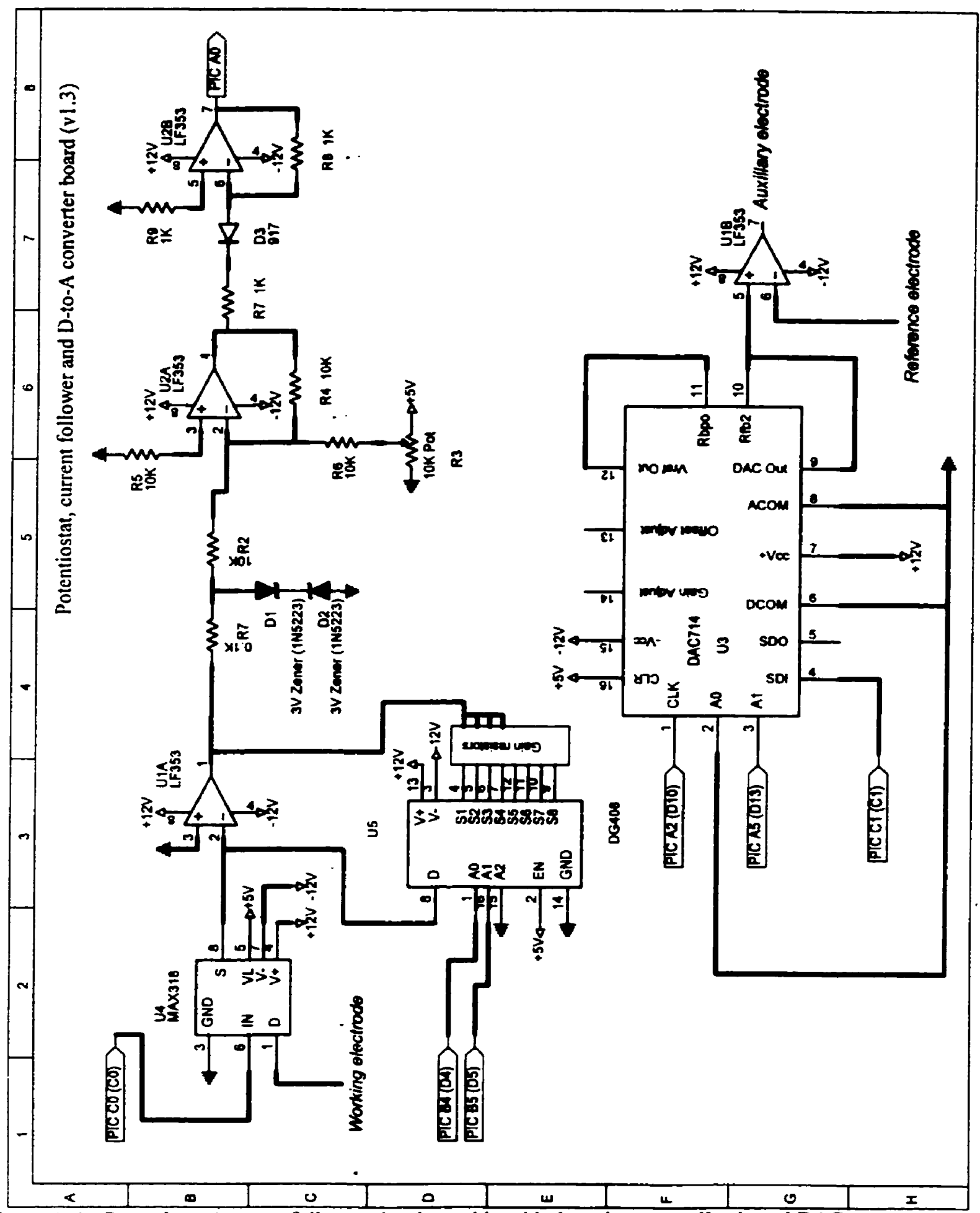

Figure A.t - Potentiostat/current follower circuit used in with the microcontroller-based DAC system. 


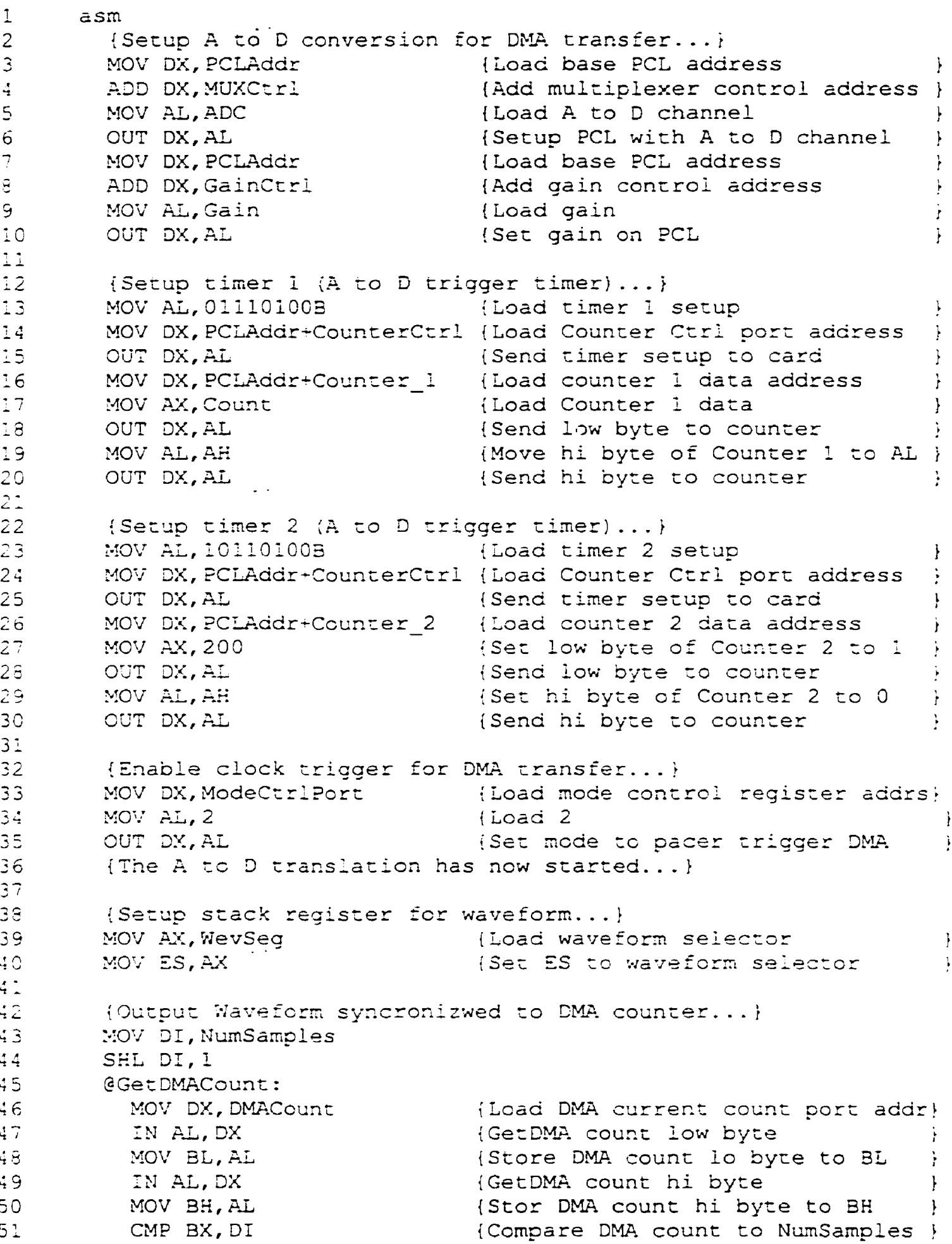


JA aDone

AND BX, SEEEE

MOV CX, ES: [BX]

CMP SI, BX

JE @GetDMACount MOV SI, BX

MOV DK, DEOAACdE

MOV AL, CI

OUT DX, AL

MOV AI, CE

INC DX

OUT DX, A.L

JMP egetDMacount

cDone:

enc; iasm;
IIf it is above, DMA is don \}

\{Even BX's point to start of word\}

(Retrieve indexed waveform word

lCompare old voltage to new

if same, don't bother settir:g it)

\{Save voltage to compare later \}

lLoad $D$ =o A low byte address

iMove lo-byte of voltage to AL

ISend waveform voltage lo byte p

iMove hi-byte of voltage to AL

ILoad A to D hi byte address

S Send waveform voliage is byce! 


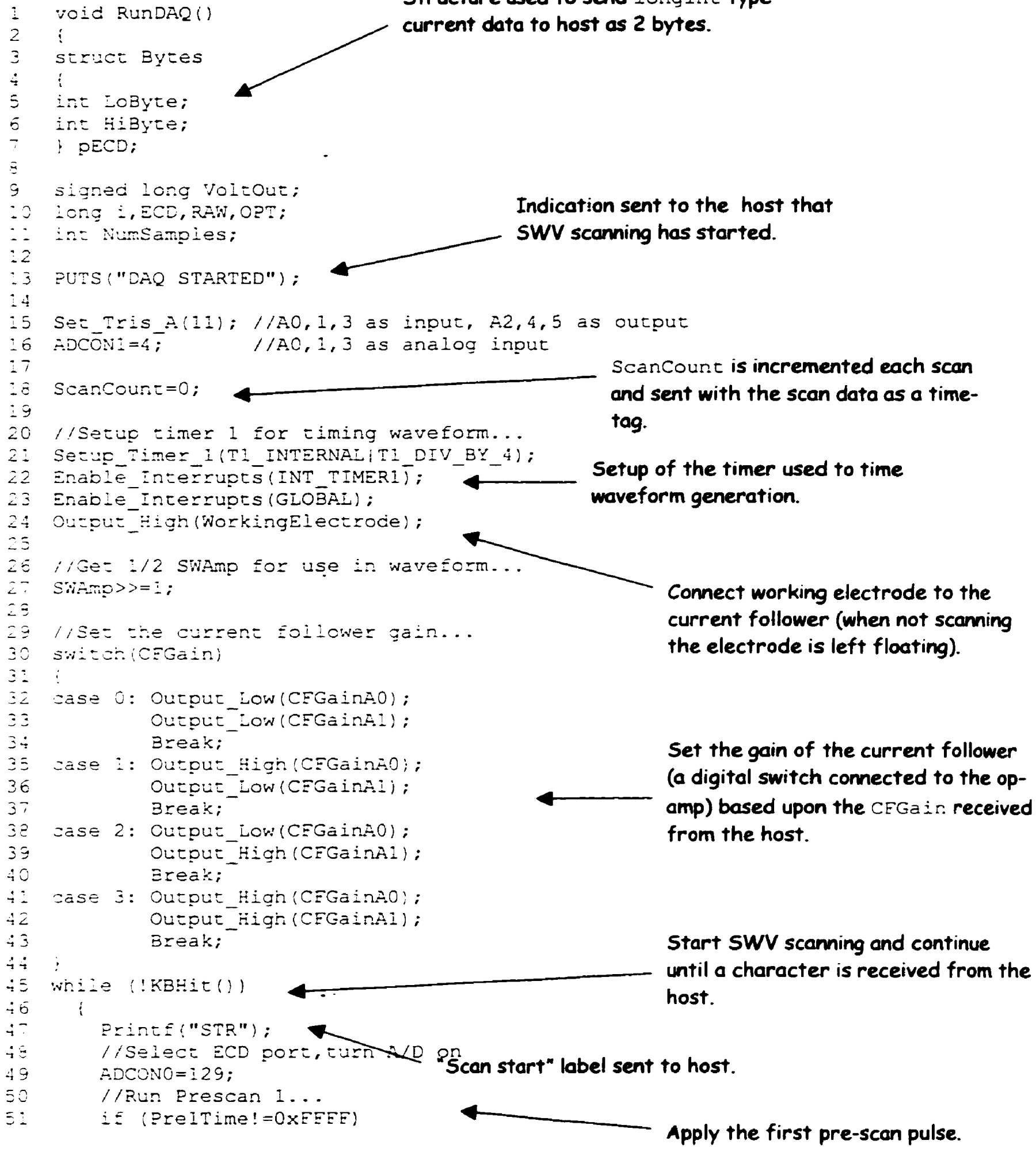

$\begin{array}{ll}50 & \text { / Run Prescan } 1 \ldots \\ 5: & \text { is (PreITime!=0XEEEF) }\end{array}$

$\begin{array}{ll}50 & \text { //Run Prescan } 1 \ldots \\ 5: & \text { iE (PreITime!=0XEEEE) }\end{array}$

Indication sent to the host that SWV scanning has started.

Scancount is incremented each scan and sent with the scan data as a timetag.

Setup of the timer used to time waveform generation.

Connect working electrode to the current follower (when not scarning the electrode is left floating).

Set the gain of the current follower (a digital switch connected to the opamp) based upon the CFGair. received from the host.

Start SWV scanning and continue until a character is received from the host.

"Scan start" label sent to host.

Apply the first pre-scan pulse. 


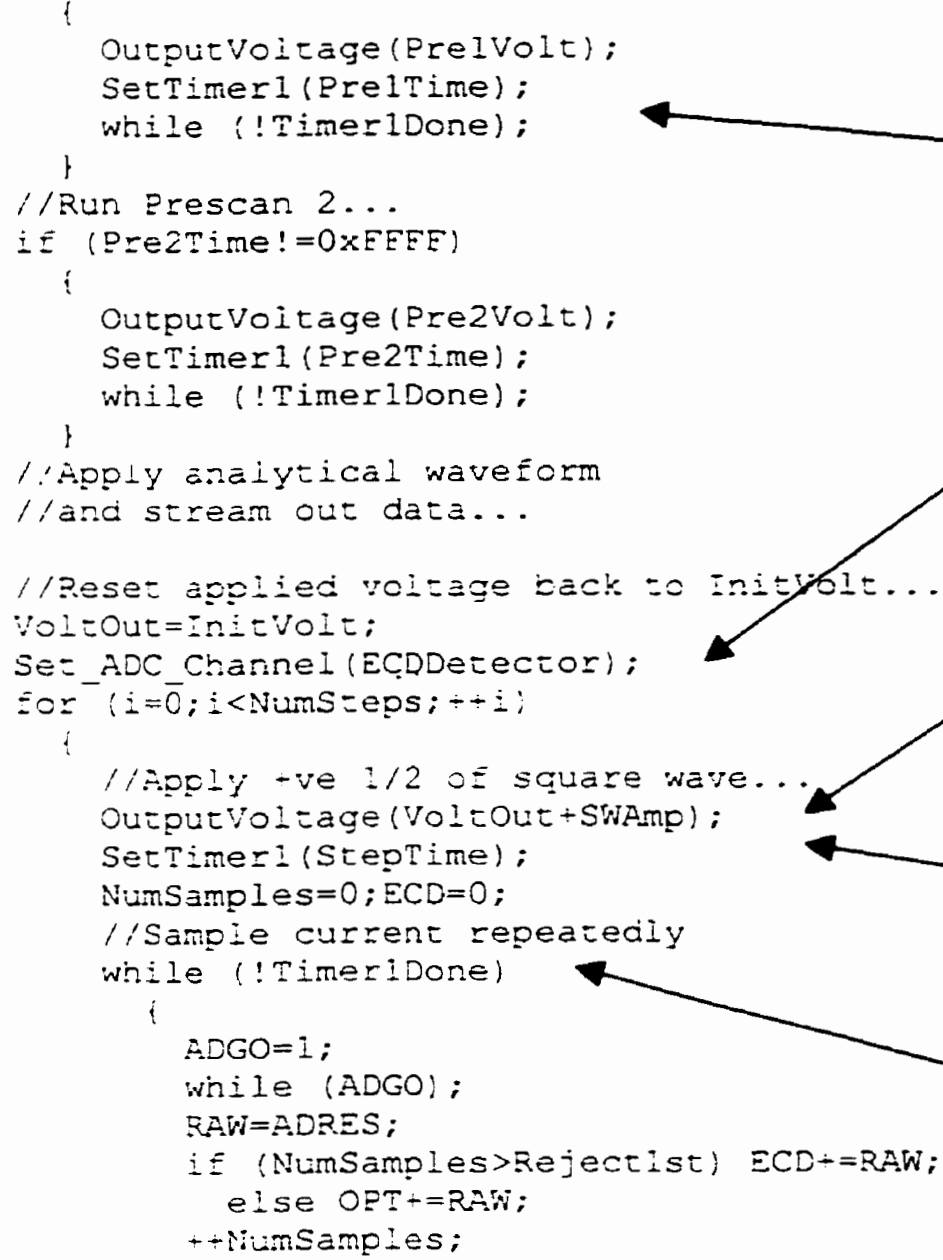

After the timer has been set, an interrupt routine will be called when it finishes which will set $T$ imeriDone to TRUE.

Apply the SWV scan.

Vol tout holds the DC ramp potential and each square-wave pulse adds or subtracts SWAmp to superimpose a square-wave on the DC ramp.

Outputvol tage is a routine (not shown here) that sends a voltage to a serial D-to-A converter attached to the PIC.

While waiting for the square-wave period timer to finish, collect and sum current samples (and keep track of how many were taken - NumSamples).

Type the current data $a S P E C D$ and send the data to the host as two bytes (its high and low bytes).

Apply second half of square-wave and similarly collect and send current data. 

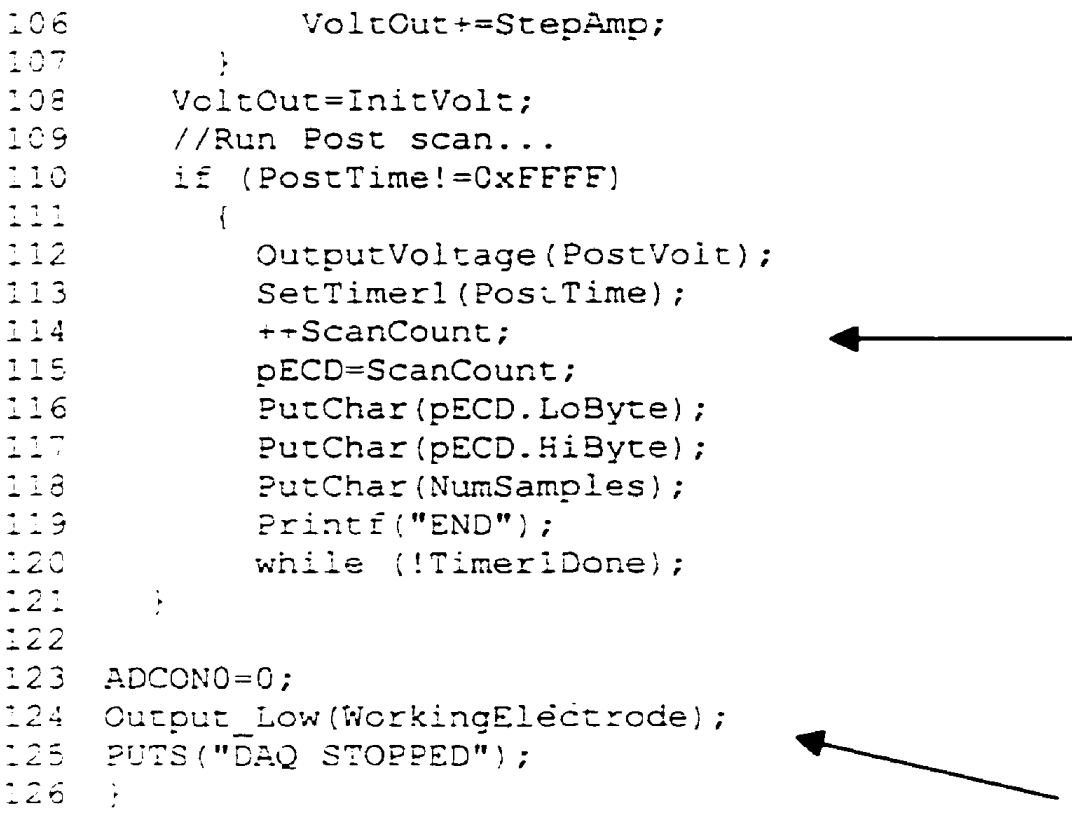

Apply post-scan pulse. Also, while waiting for the timer to expire, send the additional scan information ScanCount, NumSamples (\# of samples acquired during each squarewave puise) and "END" which acts as a terminator for the scan data.

If a character is received from the host, it will break out of SWV scanning, disconnect the working electrode from the current follower (i.e. allow it to float) and indicate to the host that scanning has stopped. 
Listing A.3 - Delphi 4.0 code used to parse incoming scan data received from microcontroller.

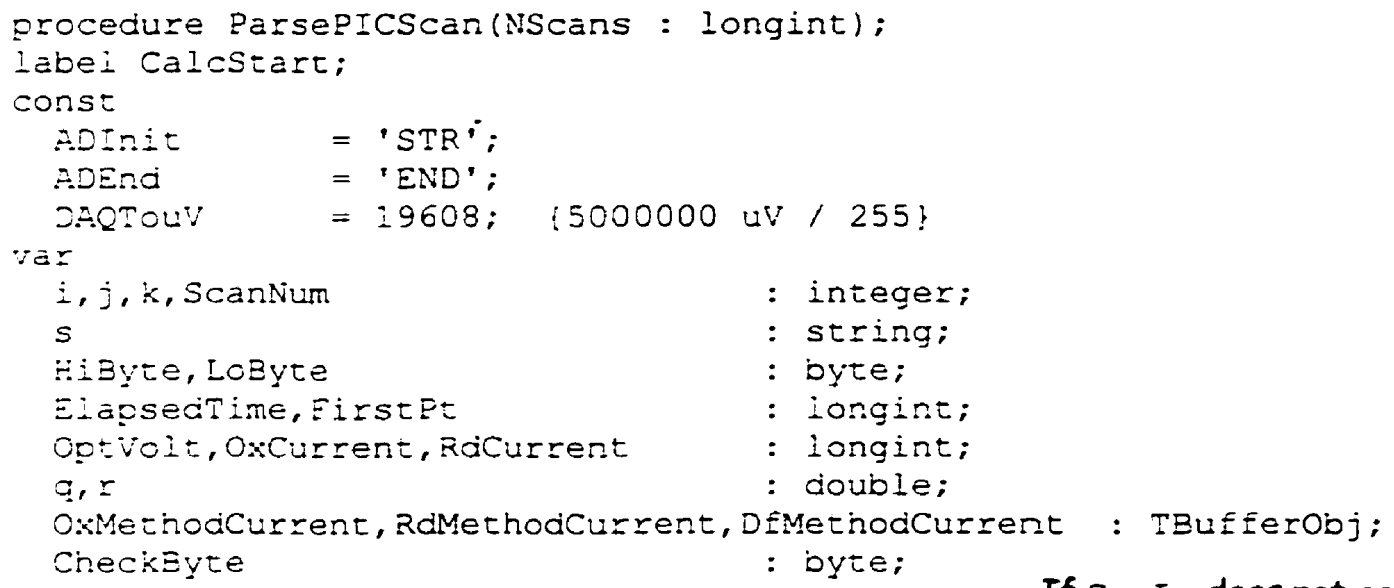

Find the start ( $i$ ) and end $(j)$ of the first SWV scan contained in Com In.

If "END" appears before "STR" this indicates an incomplete scan exists in ComIn, get rid of it and exit the procedure.

Get the number of current samples obtained for each square-wave.

NumeCDSampies : $=$ Ord $(\operatorname{Com} I n[j-i]) ;$

Scarnum: $=i+($ (Ord (ComIn[j-3]) or (Ord(Cominlj-2]) shl 8)));

ElassedTime: =ScanNum -TotalScanTime; $s:=\operatorname{cocy}(\operatorname{Com} I n, i+3, j) ;$

Delece (ComIn, 1, Pos (ADEnd, ComIn) +3);

:E Scancycle=NumScans then

Scancycle:=i else inc(scancycle);

Eor $::=0$ to (NumSamples-1) do

begin

dec(RoScans [0, i], RdScans [Scancycle, i]);

$\operatorname{dec}(0 x \operatorname{scans}[0, \dot{1}]$, OxScars [ScanCycle, i]);

jozyte:=0rd $(s[i+1+4 !)$;

$\because 3 y t e:=0 r d(s[2+i-4 i)$;

O:Curren: =(Losyte or (HiByte shl 8) ):

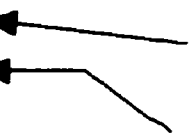

Get the Scanium and calculate the ElapsedTime

LoByte: $=\operatorname{Ord}(s[3+i+4])$; Fisyce: $=$ Ord $(s[4+i * 4])$; RcCurrent: $=($ Eobyte or (HiByte shl 8$)$ ) Rciscans [ScanC $y c i e, i]:=$ RdCurrent; OxScans [ScanCycle, i] : =0xCurrent; inc(RdScans [0,i], RdCurrent);

Copy portion of Comin containing current data to $s$ and delete scan from Com Ir.

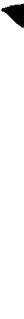
$r$

- Maintain the running scan average.

Get the 2 bytes of the forward current and recombine them.

Get the 2 bytes of the reverse current and recombine them. 

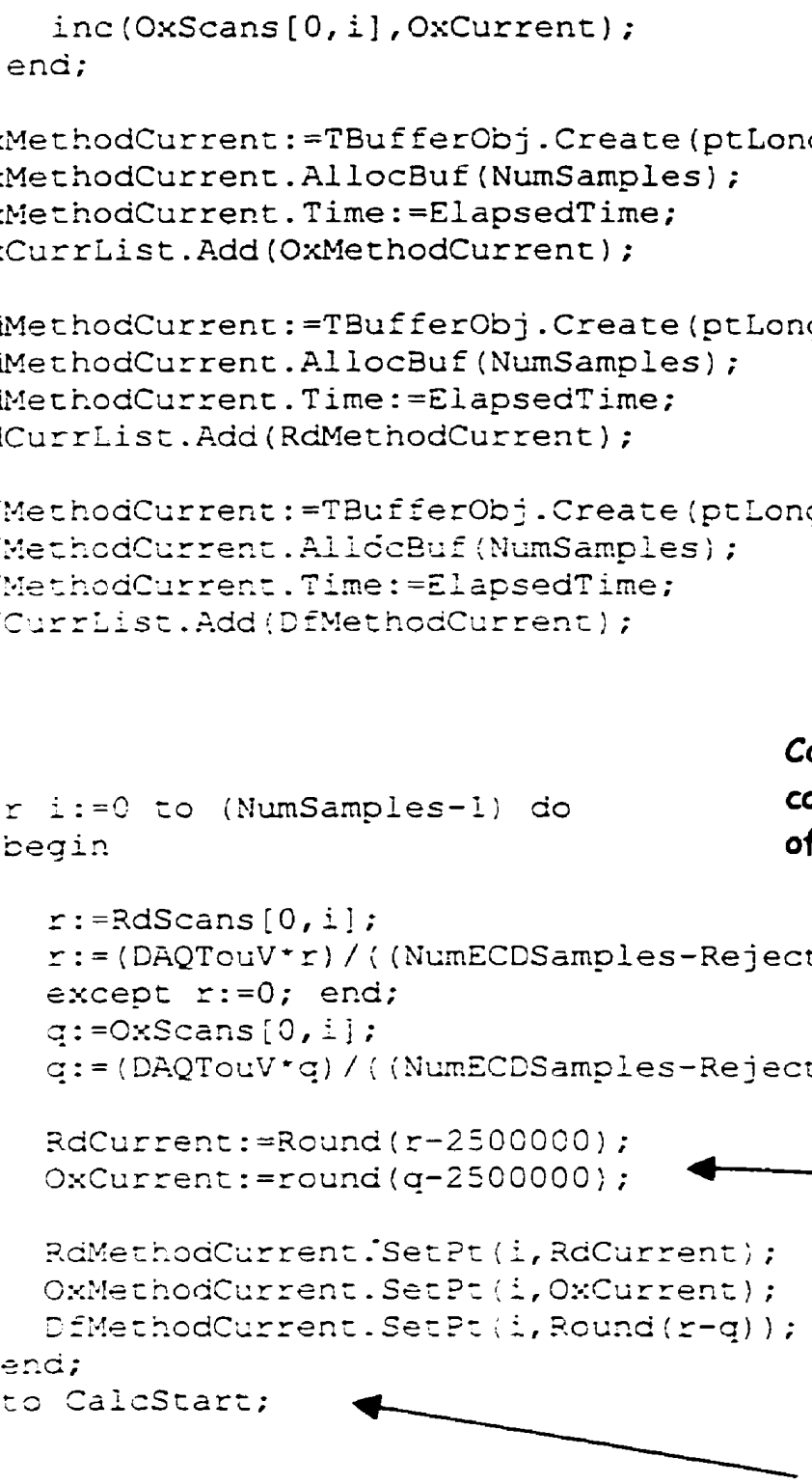

Convert the 0-255 scan data to $\mu V$ units and calculate average current based upon the number of current samples aquired/square wave.

Allocate memory for to hold the scan dato as forward, reverse and difference voltammograms and add these buffer objects to a list containing all captured voltammograms. Another procedure discussed later quantitates these scans.

Offset the current data by -2.5 volts so that $O$ volts corresponds to a value of zero.

\}

Calculate the difference current and enter all three current values into the voltammogram buffer object.

Go back to the start of the procedure and process the next scan contained in ComIn. 
Listing A.4 - Delphi 4.0 code used to quantitate stored voltammograms.

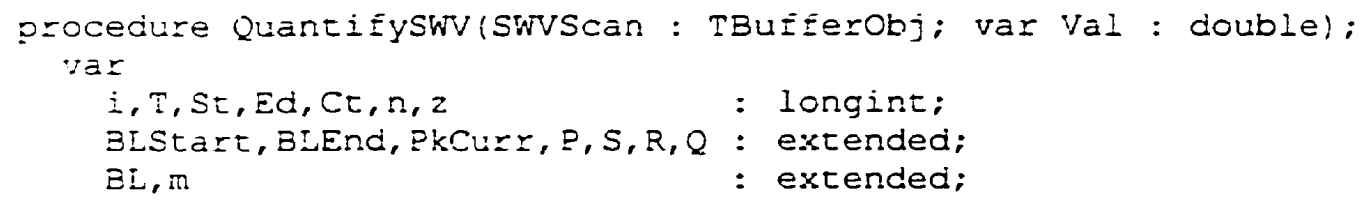

The center of the SWV peak (identified by the user)

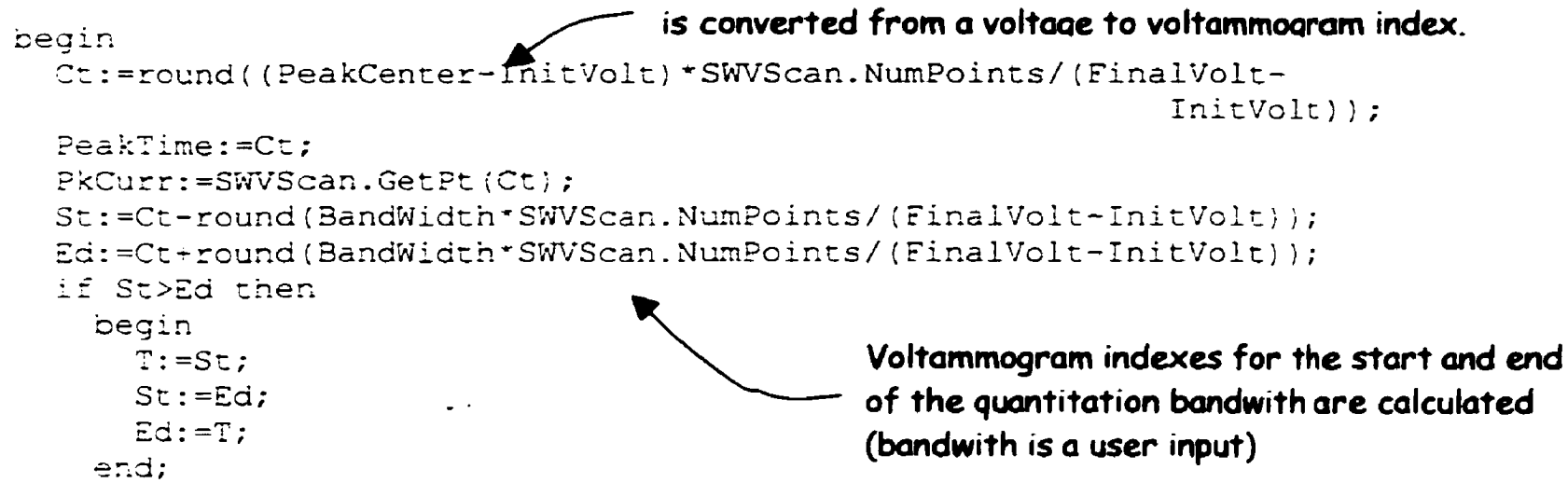

Voltammogram indexes for the start and end of the quantitation bandwith are calculated (bandwith is a user input)

This section of code calculates the equation for the baseline drawn across the base of the SWV peak.

(See Section 2.3.4)

This section of code generates a single current value for the voltammogram based upon the user-selected quantitation method 
UsePkAvg is a Boolean value indicating

If the peak baseline has been enabled, the baseline equation is used.

VaI: $=($ PkCurr $(m *(C t-S t)+$ BLStart $)) / 1000000$

else Val:=PkCurr/1000000;

end:

Enc; 
References:

${ }^{1}$ Tiselius. A.W.K.. Scientairum Upsaliensis, 7, 1937.

${ }^{2}$ Hjertén. S., Chromatogr. Rev., 9, 1967, 122-219.

${ }^{3}$ Jorgenson. J. W. and Lukacs. K. D.. Anal. Chem., 53, 1981, 1298.

+ Terabe. S.. Otsuka. K.. et al.. Anal. Chem., 56, 1984, 111-113.

' Cikalo. M.G., Bartle. K.D.. Robson. M.M., Myers, P.. Euerby, M.R.. Analyst, 123. 1008. 87R-102R.

${ }^{6}$ Christiansen. L.. Wher, T.. Hansen. D.. Spectra, 18, 1990, 35-38.

${ }^{7}$ Grossman. P. D., Soane, D. S.. J. Chrom, 559, 1991, 257-266.

${ }^{8}$ Altria. K. D.. Fabre. H.. Chromatographia. 40. 1995. 313-320.

${ }^{9}$ Altria. Kevin. D. LC-GC, 17. 1999. 28-35.

${ }^{10}$ Chen. D.Y.. Dovichi. N.J.. J.Chrom. B, 657. 1994. 265-269.

"Smith. R. D.. Olivares. J. A.. Anal. Chem. 60. 1988. 436-441.

12 LaCourse. R.W.. Dasenbrock. C.O.. Advances in Chromatography. Marcel Dekker. New York. 1998. 189-232.

${ }^{13}$ Wallingford, R. A. and A. G. Ewing, Anal. Chem. 59. 1987. 1762-1766.

${ }^{14}$ Wallingford. R. A. and A. G. Ewing. Anal. Chem.,60, 1988, 258-263.

I5 Wallingford. R. A. and A. G. Ewing. Anal. Chem. 60, 1988. 1972-1975.

${ }^{16}$ A. L. Knecht. E. J. Guthrie and J. W. Jorgenson, Anal. Chem., 56. 1984, 479-482.

${ }^{17}$ Wallingford. R. A. and A. G. Ewing, Anal. Chem., 61. 1989, 98-100

${ }^{18}$ Huang. X. and R. N. Zare. Anal. Chem., 62, 1990. 443-446. 
${ }^{19}$ O'Shea. T. J., Greenhagen R.D., J. Chromatogr., 593, 1992, 305-312.

${ }^{20}$ Kok, W. T. and Sahin, Y., Anal. Chem., 65, 1993, 2497-2501.

${ }^{21}$ Park. S.. Lunte, S. M.. et al.. Anal. Chem. 67, 1995, 911-918.

22 Hu. S., Wang. Z. L.. et al., Anal. Chem., 69, 1997, 264-267.

23 Sloss. S.. Ewing. A.G.. Anal. Chem. 63. 1993. 577-581.

${ }^{24}$ Huang. X.. Zare. R. N.. Anal. Chem. 63. 1991. 189-192.

${ }^{25}$ Lu. W., Cassidy. R. M.. Baranski. A. S., J. Chromatogr. 640. 1993. 433-440.

${ }^{26}$ Chen. M. C. and Huang. H. J.. Anal. Chem. 67. 1995. 4010-4014.

${ }^{27}$ Fermier. A. M.. Gostkowski. M. L.. Colon, L.A., Anal. Chem., 68. 1996, 1661-1664.

${ }^{28}$ Matysik. F. M.. Journal Of Chromatography A., 742. 1996, 229-234.

29 Ye. J. and Baldwin. R. P., Anal. Chem., 65, 1993. 3525-3527.

${ }^{30}$ Durgbanshi. A. and Kok. W. T.. J. Chrom. A, 798. 1998, 289-296.

${ }^{31}$ Voegel. P. D. and Baldwin. R. P., Electrophoresis, 18. 1997. 2267-2278.

32 Yik. Y. F.. Li. S. F. Y.. Trends Anal. Chem., 11, 1992. 325-332.

${ }^{33}$ Curry. P. D.. Engstrom. S. C. E.: Ewing. A. G. Electroanalysis. 3. 1991. 587-596.

it Virtanen. R.. Acta Polytech. Scad. 123. 1974. 1-12.

${ }^{35}$ Haber. C.. Silvestri. I., Roosli. S.; Simon, W. Chimia, 45, 1991. 117-121.

${ }^{36}$ Kappes. T.. Schnierle. P.. Hauser. P. C.. Analytica Chimica Acta.. 350. 1997. 141-147.

${ }^{37}$ Mikkers. F.E.P., Everaerts. F.M.. Verhegen. Th.P.E.M, J. Chromatog., 169, 179. 11-20.

${ }^{38}$ Huang, X., Luckey, J.A.. Gordon. M.J., Zare. R.N., Anal. Chem. . 61, 1989. 766.

${ }^{39}$ Goto. M. and Shimada. K.. Chromatographia. 21, 1986, 631-634. 
${ }^{+0}$ Caudill, L.W.. Ewing, A.G., Jones, S., Wightman, R.M., Anal. Chem., 55, 1983, $1877-$ 1881 .

${ }^{+1}$ White. J.G., St. Claire III, R., L., Jorgenson, J.W., Anal. Chem., 58. 1986, 293-298.

${ }^{12}$ Park, S., McGrath. M..J., Smyth. M.R., Diamond, D., Lunte, C.E., Anal. Chem. 69, 1997. 2994-3001.

${ }^{43}$ Ferris. S.S., Lou. G.. Ewing, A.G.. J. Microcol. Sep.. 6. 1994. 263-268.

it Wightman. R.M.: Wipf, D.O. Electroanalytical Chemistry (Edited by A.J. Bard), Vol. 5 , Dekker. New York. 1988. 308.

${ }^{45}$ Osteryoung. J.G.. Osteryoung. R.A.. Anal. Chem., 57. 101A-1 10A.

${ }^{46}$ Fung. Y.S.. Mo. S.Y.. Analytical Sciences. 10. 1994, 179-180.

${ }^{47}$ Roush. J.A.. Anderson. M.R.. J. Liq. Chromatogr.. 16. 1993. 3887-3901.

${ }^{48}$ Samuelsson. R.. O`Dea. J.. Osteryoung. J.. Anal. Chem., 52. 1980. $2215-2216$.

${ }^{49}$ Kounaves. S.P.. Young. J.B., Anal. Chem. 61, 1989, 1469-1472.

${ }^{50}$ Cassidy. R.. Janoski. M.. LC-GC. 10. 1992, 692-696.

sl Zhong. M.. Lunte. S.M.. Anal. Chem. 68, 1996, 2488-2493.

52 Lipkowski. J.. Philip. N.R.. Adsorption of Molecules at Metal Electrodes. VCH Publishers. 1992.

53 Baranski. A.S.. Moyana. A.. Langmuir. 12. 1996. 3295-3304.

${ }^{54}$ Baranski. A.S.. Norouzi. P. Can. J. Chem., 75, 1997. 1736-1749.

\$5 Wieckowski. A.. J. Electroanal. Chem.. 352. 1993, 313-320.

${ }^{56}$ Baranski. A.S.. Krogulec. T.. Nelson. L.J., Norouzi, P. Anal. Chem., 70. 1998, 28952901.

${ }^{57}$ Gerhardt. G.C.. Salisbury. MacNeil, J.D.. J. AOAC, 77. 1994. 334-337.

${ }^{58}$ Pons, S.. Fleishman, M., Anal. Chem., 59. 1987, 1391 A-1399A. 
${ }^{59}$ Johnson. D.C.. LaCourse, W.R., Anal. Chem., 62, 1990, 589A-597A.

${ }^{60}$ Austin, D.S., Polta, J.A., Polta, T.Z., Tang, A.P.C., Cabelca, T.D.. J Electranal. Chem., 168. 1984. 227-248.

${ }^{61}$ Hudson. J.C.. Golin. M.. Malcolm, M., Can. Soc. Forens. Sci., 28, 1995, 137-152.

${ }^{62}$ Hudson. J.C., Golin. M., Malcolm, M., Can. Soc. Forens. Sci., 31. 1998. 1-29.

${ }^{63}$ Personal communication with John Hudson. R.C.M.P. Forensic Laboratory, Regina, SK. Canada.

${ }^{64}$ Fanali. S.. J. Chrom. A, 792, 1997, 227-267.

${ }^{65}$ Personal communication with Jon Bailey. All enantiomeric separations shown and in this report were developed by Jon Bailey. University of Saskatchewan (Saskatoon, SK).

${ }^{66}$ Personal communication with Stephan Lee (Canadian Food Inspection Agency, Saskatoon. SK) regarding an HPLC-UV method currently under development.

${ }^{67}$ Chien. R. L.. Burgi. D. S.. Anal. Chem.. 64, 1992, 489A-496A.

${ }^{68}$ Martinez R.I. Villanueva. V.R.M., Alvarez-Coque. G.M.C.. Anal. Chem.. 71. 1999. 319-326.

${ }^{64}$ Chevolleau. S.. Tulliez J.. J. Chrom. A. 715. 1995. 345-354.

${ }^{70}$ Boison. J.O.. Keng. L.J.. J. .AOAC Int.. 77. 1994. 558-564. 